\title{
MODELAGEM E CONTROLE ÓTIMO DE UM ROBÔ QUADRÚPEDE
}




\section{ALAIN SEGUNDO POTTS}

\section{MODELAGEM E CONTROLE ÓTIMO DE UM ROBÔ QUADRÚPEDE}

Tese apresentada à Escola Politécnica

da Universidade de São Paulo para

obtenção do título de:

Doutor em Engenharia

São Paulo

2011 


\section{ALAIN SEGUNDO POTTS}

\section{MODELAGEM E CONTROLE ÓTIMO DE UM ROBÔ QUADRÚPEDE}

Tese apresentada à Escola Politécnica

da Universidade de São Paulo para

obtenção do título de:

Doutor em Engenharia

Área de concentração:

Engenharia de Sistemas

Orientador:

Prof. Dr. José Jaime da Cruz

São Paulo 
Este exemplar foi revisado e alterado em relação à versão original, sob responsabilidade única do autor e com a anuência de seu orientador.

São Paulo, de novembro de 2011.

Assinatura do autor

Assinatura do orientador

\section{FICHA CATALOGRÁFICA}

Segundo Potts, Alain

Modelagem e controle ótimo de um robô quadrúpede / $A$.

Segundo Potts. - ed.rev. -- São Paulo, 2011.

$150 \mathrm{p}$.

Tese (Doutorado) - Escola Politécnica da Universidade de São Paulo. Departamento de Engenharia de Telecomunicações e Controle.

1. Modelagem cinemática 2. Modelagem dinâmica 3. Robô quadrúpede 4. Controle ótimo I. Universidade de São Paulo. Escola Politécnica. Departamento de Engenharia de Telecomunicações e Controle II. t. 


\section{Dedicatória}

...à minha querida mãe Beatriz,

ao meu pai Manuel e à minha irmã Heydi, à minha amada esposa Giselle, e em especial, ao meu pequeno filho Daniel. 


\section{Agradecimentos}

Em primeiro lugar a Deus, por me orientar sempre o caminho a seguir na vida e me ajudar a chegar até aqui.

Ao meu orientador, professor Dr. José Jaime da Cruz por me aceitar como seu aluno, pela sua dedicação apresentada na condução desta pesquisa e pela relação de amizade construída ao longo destes anos.

Ao Brasil, e especialmente à Universidade de São Paulo que me abriram as portas e deram a oportunidade de aumentar meus conhecimentos e desenvolver este projeto de pesquisa.

À minha familia, e em particular aos meus pais que sempre se preocuparam por meus estudos e acreditaram no meu sucesso.

À minha esposa Giselle pelo seu apoio e compreensão e ao me filho Daniel por ser minha maior fonte de forças e inspiração.

Ao professor Wildor e especialmente a Guillermo por toda sua ajuda na minha vinda ao Brasil e entrada na USP.

Aos meus amigos do LAC, Átila Madureira Bueno, Rodrigo Alvite Romano, Reginaldo Inojosa, e Paulo Rocha por seus conselhos, ajuda e amizade.

Ao professor Dr. Tarcisio Antonio Hess Coelho, pela ajuda e sugestões na modelagem do robô.

À CAPES pelo suporte econômico nestes anos de pesquisa.

Aos amigos e professores do Departamento de Automação e Computação do Instituto Superior Politécnico "José Antonio Echeverría"(CUJAE), onde inicie a minha carreira acadêmica.

À Patricia pela ajuda na revisão do texto.

A todos os professores que ao longo da minha vida me ensinaram e formaram a base dos meus conhecimentos. 
"Um pouco de ciência nos afasta de Deus.

Muita, nos aproxima."

(Louis Pasteur) 


\section{Resumo}

O presente trabalho visa à modelagem e ao controle ótimo de um robô quadrúpede autônomo. Devido a variações na topologia e nos graus de liberdade do robô ao longo do seu movimento, duas abordagens diferentes de modelagem foram consideradas: na primeira, foi considerado o robô com pelo menos duas pernas suportando seu corpo ou plataforma e, na segunda, considerou-se o modelo de uma perna no ar. Em ambos os casos, apresentou-se a solução dos problemas cinemáticos de posição direta e inversa por meio da parametrização de Denavit-Hartenberg. Analisaramse também os problemas cinemáticos de velocidade e suas singularidades através da Matriz Jacobiana, e ainda obtiveram-se os modelos dinâmicos do sistema utilizandose o Principio do Trabalho Virtual e o método iterativo de Newton-Euler para a plataforma e as pernas, respectivamente. A partir destes modelos dinâmicos, desenvolveu-se um algoritmo de otimização das perdas de energia elétrica dos motores das juntas. Neste sentido, utilizou-se a estratégia do controle independente por junta. Estratégia esta que, junto com a discretização no tempo do modelo do sistema, permitiu transformar o problema inicial de otimização para cada junta em outro de Programação Quadrática bem mais simples de ser resolvido. Depois de resolver estes problemas, para levar em conta as interações entre as dinâmicas das várias juntas, procedeu-se à busca de um ponto fixo ou mínimo global que caracterizasse a energia total gasta no movimento do sistema. Finalmente, realizada a demonstração e a análise de convergência do algoritmo, este foi testado no controle da andadura (gait) do robô Kamambaré. Como resultado do teste, observou-se o bom desempenho da formulação e a viabilidade de sua implementação em sistemas reais.

Palavras-chaves: Modelagem cinemática, modelagem dinâmica, robô quadrúpede, controle ótimo. 


\section{Abstract}

The present work aims the modeling and optimal control of an autonomous quadruped robot. Due to variations in the topology and the degree of freedom of the robot during its motion, two different modeling approaches were considered: firstly, the robot was considered with at least two legs supporting its body or platform and, second one, was considered the model of a leg in the air. In both cases, was presented the solution of the direct and inverse kinematic problem of position through the Denavit-Hartenberg parameterization. Were analyzed also, the kinematic problem of speed and the singularities through the Jacobian matrix, and was also obtained the dynamic model of the system using the Principle of Virtual Work or the d'Alembert method and the iterative Newton-Euler method for the platform and legs, respectively. From these two dynamic model, were developed an algorithm for optimizing the power losses of the motors that driven the joints. In this sense, was used the strategy of independent control for each joint. Such a strategy, along with the discretization in time of the system model, has helped to change the initial optimization problem for each joint in a Quadratic Programming Problem, more simpler to solve. After solving these problems, and to take into account the interactions between the dynamics of various joints, was proceeded to search for a fixed point or a global minimum that would characterize the total energy spent in moving for the system. Finally, held the demonstration and analysis of convergence of the algorithm was tested in the control of gait of the Kamambaré robot. As a result of the test, we observed the good performance of the formulation and the feasibility of its implementation in real systems.

Key words: kinematical model, dynamical model, quadruped robot, optimal control. 


\section{Lista de Figuras}

1.1 Primeiro robô da Unimation construído no ano 1961. Fonte: RobotWorx $(2011) \ldots \ldots \ldots \ldots \ldots$

1.2 Robô quadrúpede TITAN VIII desenvolvido pelo ITT. Fonte: Robotics Lab $(2011) \ldots \ldots \ldots \ldots$

1.3 Robô RiSE versão 1.5 Fonte: $\operatorname{Kod} * \operatorname{Lab}(2011)$. . . . . . . . . . . . 4

1.4 Robô RiSE versão 3: Fonte: Haynes et al. (2009). . . . . . . . . . . . 4

1.5 Robô Kamambaré . . . . . . . . . . . . . . . . . . . . . . . . . . 7

1.6 Comportamento do mercado da robótica de 1995 até o ano 2025. Fonte: EurAtiv.com $(2005) \ldots \ldots \ldots \ldots$. . . . . . . 8

2.1 Projeto mecânico do robô Kamambaré. . . . . . . . . . . . . . . . . . 13

2.2 Perna do Kamanbaré . . . . . . . . . . . . . . . . . . . . . . . 14

2.3 Definição do ângulo $\theta_{1_{l}} \ldots \ldots \ldots \ldots \ldots \ldots \ldots$

2.4 Rotações nos eixos Z-Y'-Z". Fonte Harib e Srinivasan (2003a). . . . . 18

2.5 Espaço de trabalho do ponto ${ }^{O} P$ da plataforma para $0.24 \leq \psi_{P} \leq 1.32$ quando as pernas 1 e 3 estão fixas à superfície de apoio. $\left(\mathcal{W}_{P}=\right.$

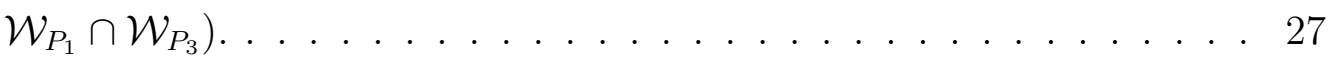

2.6 Espaço de trabalho da garra $l$ no ar. . . . . . . . . . . . 30

3.1 Mapeamento entre os diferentes espaços de estados. . . . . . . . . . . 31

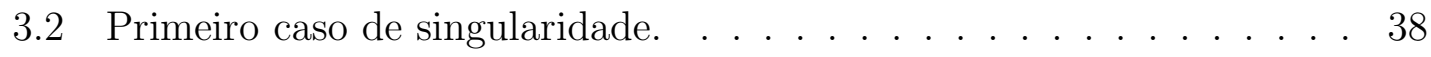

3.3 Segundo caso de singularidade. . . . . . . . . . . . . 38 
3.4 Condição de singularidade para $\theta_{P}=0$ (a) e $\theta_{P}=-\frac{\pi}{2}(\mathrm{~b}) \ldots . . . \quad 40$

3.5 Exemplo de singularidade da perna 1 no ar para $\theta_{2_{1}}=1.274$ e $\theta_{3_{1}}=-\frac{\pi}{4}$. 44

3.6 Exemplo de singularidade da perna 1 no ar para $\theta_{2_{1}}=-1.274 \mathrm{e}$ $\theta_{31}=-\frac{\pi}{4} \ldots \ldots \ldots \ldots \ldots \ldots 4 \ldots \ldots . \ldots \ldots$

3.7 Singularidade na fronteira do espaço de trabalho da perna 1 no ar. $\left(\theta_{2_{1}}=-\frac{\pi}{2}, \theta_{3_{1}}=0\right)$.

3.8 Singularidade na fronteira do espaço de trabalho da perna 1 no ar. $\left(\theta_{1_{1}}=\frac{\pi}{2}, \theta_{2_{1}}=\theta_{3_{1}}=0\right)$.

3.9 Singularidade na fronteira do espaço de trabalho da perna 1 no ar. $\left(\theta_{1_{1}}=\pi, \theta_{2_{1}}=\theta_{3_{1}}=0\right) . \ldots \ldots . . \ldots . . . \ldots 46$

4.1 Eixos de rotação da plataforma . . . . . . . . . . . . . 53

4.2 Esquema de forças e torques para uma perna do Kamambaré . . . . . 59

5.1 Diagrama eletro-mecânico de uma junta. . . . . . . . . . . . . . 63

5.2 Controle independente por junta, utilizando como variável manipulada o torque do motor. . . . . . . . . . . . . . . 65

5.3 Ciclo interno do algortimo de otimização. . . . . . . . . . . . . . . . 72

5.4 Ciclo externo do algortimo de otimização. . . . . . . . . . . . . 73

6.1 Diferentes tipos de andaduras segundo Hildebrand (1989). . . . . . . 79

6.2 Andadura do Kamambaré. o Perna no ar. • Perna de apoio. . . . . . 80

6.3 Modelo mecânico . . . . . . . . . . . . . . . . . . . . . . 81

6.4 Fluxograma de controle para um ciclo básico da andadura. . . . . . . 84

6.5 Posição angular de cada junta durante a etapa I . . . . . . . . . . . . 86

6.6 Deslocamento do centro da plataforma durante a etapa I . . . . . . . 86

6.7 Rotações em tornos dos eixos do sistema $\{P\}$ durante a etapa I . . . 87

6.8 Torques de carga de cada junta durante a etapa I . . . . . . . . . . . 87 
6.9 Corrente de armadura de cada junta durante a etapa I . . . . . . . . 88

6.10 Tensão de armadura de cada junta durante a etapa I . . . . . . . . . 88

6.11 Potência total consumida pelos motores na etapa I. . . . . . . . . . . 89

6.12 Erro entre as soluções de duas iterações consecutivas para o conjunto das juntas $\mathcal{A}$ na etapa I . . . . . . . . . . . . . . . . . . . . . . . 89

6.13 Corrente total consumida pelos motores das juntas $\mathcal{A}$ na etapa I . . . 90

6.14 Posição angular de cada junta durante a etapa II . . . . . . . . . . . 90

6.15 Coordenadas do ponto $A_{2}$ durante a etapa II . . . . . . . . . . . . . 91

6.16 Torques de carga de cada junta durante a etapa II . . . . . . . . . . . 91

6.17 Corrente de armadura de cada junta durante a etapa II . . . . . . . . 92

6.18 Tensão de armadura de cada junta durante a etapa II . . . . . . . . . . 92

6.19 Potência total consumida pelos motores na etapa II. . . . . . . . . . . 93

6.20 Erro entre as soluções de duas iterações consecutivas para o conjunto das juntas $\mathcal{A}$ na etapa II . . . . . . . . . . . . . . . . . . . . . . . . . 93

6.21 Corrente total consumida pelos motores das juntas $\mathcal{A}$ na etapa II . . 94

6.22 Variação da região de deslocamento de $\theta_{4_{1}}$ para diferentes $t_{f}$. . . . . 95

6.23 Variação da corrente consumida na junta $\theta_{4_{1}}$ para diferentes $t_{f} . \quad$. . 95

6.24 Variação da corrente total consumida pelos motores da perna $l=2 . \quad$. 96

6.25 Energia cinética ao longo da andadura. . . . . . . . . . . . . . . . 97

6.26 Esquema de camadas de controle. . . . . . . . . . . . . . . . . . . 98

D.1 Motor estilo Mabuchi RS-540 de 3 pólos de armadura. Fonte: Tros-

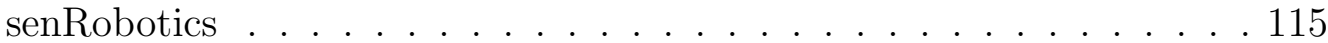

E.1 Redução por engrenagem Tamiya 70103. Fatores de redução de 101:1, 269:1 e 719:1. Fonte: Robotics Electronics . . . . . . . . . . . . 116

F.1 Especificações físicas. Fonte: Eletronica-Industrial ........ 117 
F.2 Tempo de descarga em função do consumo de corrente do circuito. Fonte: Eletronica-Industrial . . . . . . . . . . . . . . . . . . 117

F.3 Curvas de descarga da bateria. Fonte: Eletronica-Industrial . . . . . 118

F.4 Especificações elétricas. Fonte: Eletronica-Industrial . . . . . . . . . 118

G.1 Sequência de movimentos do Kamambaré sob a ação do algoritmo de otimização num ciclo completo de sua andadura. . . . . . . . . . . . . 119 


\section{Lista de Tabelas}

2.1 Tabela de Denavit-Hartenberg para a solução do problema direto. . . 16

2.2 Comprimento dos ligamentos das pernas . . . . . . . . . . . . . 28

6.1 Classificações das juntas na andadura do Kamambaré. . . . . . . . . . 82

6.2 Grandezas físicas do robô . . . . . . . . . . . . . . . . . 83

6.3 Parâmetros de controle . . . . . . . . . . . . . . . 85

6.4 Resultados da otimização. . . . . . . . . . . . . . . . . 96

C.1 Amplitude de trabalho das juntas do Kamambaré . . . . . . . . . . . 112

D.1 Características do motor. Fonte: TrossenRobotics . . . . . . . . . . . 115 


\section{Lista de Símbolos}

$\alpha \quad$ Constante de Lipschitz.

$\beta_{i_{l}} \quad$ Constante de atrito relativa à junta $i_{l}$.

$\gamma_{i_{l}} \quad$ Constante de atrito relativa à junta $i_{l}$.

$\delta(\cdot) \quad$ Deslocamento virtual de $(\cdot)$

$\varepsilon_{i_{l}} \quad$ Energia elétrica consumida pelo motor C.C. da junta $i_{l}$.

$\xi \quad$ Valor de tolerância para a convergência do algoritmo.

$\phi_{P} \quad$ Angulo de Euler para a plataforma.

$\psi_{l} \quad$ Angulo de rotação da garra.

$\psi_{P} \quad$ Angulo de Euler para a plataforma.

$\rho_{c} \quad$ Densidade de um corpo rígido.

$\check{\theta}_{m_{i_{l}}} \quad$ Posição angular mínima do rotor do motor C.C. da junta $i_{l}$.

$\hat{\Theta}_{m_{i_{l}}}$ Vetor formado pelos valores discretos $\hat{\theta}_{m_{i_{l}}}\left(t_{1}\right), \hat{\theta}_{m_{i_{l}}}\left(t_{2}\right), \ldots, \hat{\theta}_{m_{i_{l}}}\left(t_{N}\right)$.

$\hat{\theta}_{m_{i_{l}}} \quad$ Posição angular máxima do rotor do motor C.C. da junta $i_{l}$.

$\theta_{i_{l}} \quad$ Posição angular da i-ésima junta da l-ésima perna do robô.

$\Theta_{m_{i_{l}}}$ Vetor formado pelos valores discretos $\theta_{m_{i_{l}}}\left(t_{1}\right), \theta_{m_{i_{l}}}\left(t_{2}\right), \ldots, \theta_{m_{i_{l}}}\left(t_{N}\right)$

$\theta_{m_{i_{l}}} \quad$ Posição angular do motor C.C. da junta $i_{l}$.

$\theta_{m} \quad$ Posição angular do rotor do motor C.C. 
$\theta_{P} \quad \hat{A}$ Angulo de Euler para a plataforma.

$\tilde{\theta}_{m_{i_{l}}} \quad$ Posição angular final do motor C.C. da junta $i_{l}$.

$\bar{\tau} \quad$ Torque máximo do sistema.

$\tau_{\text {atrito }}$ Torque relativo à força de atrito.

$\tau_{i_{l}} \quad$ Torque de carga da junta $i_{l}$.

$\tau_{m_{i_{l}}} \quad$ Torque do motor C.C. da junta $i_{l}$.

$\tilde{\tau}_{m_{i_{l}}} \quad$ Torque máximo do motor informado pelo fabricante.

$\vec{\tau} \quad$ Vetor de torques nas juntas do sistema.

$\omega_{i_{l}} \quad$ Velocidade angular do ligamento $i_{l}$.

$\omega_{p} \quad$ Velocidade angular da plataforma.

${ }^{(i+1)_{l}} \omega_{i_{l}}$ Velocidade angular do ligamento $i_{l}$ em relação ao sistema inercial $\{O\}$, expressa no sistema de coordenadas do ligamento $\left\{(i+1)_{l}\right\}$.

${ }^{O} \omega_{p_{x}},{ }^{O} \omega_{p_{y}},{ }^{O} \omega_{p_{z}}$ Componentes em $x, y$ e $z$ da velocidade angular da plataforma com relação ao sistema $\{O\}$.

$\chi_{m} \quad$ Fluxo eletromagnético do motor C.C.

$\bar{A}_{i_{l}}, \bar{B}_{i_{l}}, \bar{C}_{i_{l}}$ Matrizes de estado do motor C.C. da junta $i_{l}$.

$\mathcal{A} \quad$ Conjunto das juntas ativas.

$\left\{A_{l}\right\}$ Sistema de coordenadas fixo ao ligamento $L_{5}$ da l-ésima perna do robô.

${ }^{E_{l}} A_{x_{l}},{ }^{E_{l}} A_{y_{l}},{ }^{E_{l}} A_{z_{l}}$ Componentes em $x, y$ e $z$ do ponto de agarramento $A_{l}$ relativos ao sistema $\left\{E_{l}\right\}$.

$A_{l} \quad$ Origem do sistema de coordenadas $\left\{A_{l}\right\}$ fixo ao ligamento $L_{5}$ da l-ésima perna do robô. Coincide com o ponto de agarramento desta perna.

$A_{l}(I)$ Posição de agarramento da perna $l$ na etapa I da andadura.

$\left\{B_{l}\right\}$ Sistema de coordenadas fixo ao ligamento $L_{4}$ da l-ésima perna do robô. 
$B\left(\theta_{\mathbf{l}}\right)$ Matriz de coeficientes de Coriolis.

$B_{l} \quad$ Origem do sistema de coordenadas $\left\{B_{l}\right\}$ fixo ao ligamento $L_{4}$ da l-ésima perna do robô.

$B_{m} \quad$ Coeficiente de amortecimento viscoso do motor C.C.

$\left\{C_{l}\right\}$ Sistema de coordenadas fixo ao ligamento $L_{3}$ da l-ésima perna do robô.

$C\left(\theta_{\mathbf{l}}\right)$ Matriz dos coeficientes centrífugos.

$c \theta \quad$ Versão simplificada para representar o $\cos \theta$.

$C_{l} \quad$ Origem do sistema de coordenadas $\left\{C_{l}\right\}$ fixo ao ligamento $L_{3}$ da l-ésima perna do robô.

$\mathcal{D} \quad$ Conjunto das juntas dependentes.

$\left\{D_{l}\right\}$ Sistema de coordenadas fixo ao ligamento $L_{2}$ da l-ésima perna do robô.

$D_{l} \quad$ Origem do sistema de coordenadas $\left\{D_{l}\right\}$ fixo ao ligamento $L_{2}$ da l-ésima perna do robô.

$\left\{E_{l}\right\}$ Sistema de coordenadas fixo ao l-ésimo vértice da plataforma.

${ }^{O} E_{x_{l}},{ }^{O} E_{y_{l}},{ }^{O} E_{z_{l}}$ Componentes em $x, y$ e $z$ do ponto $E_{l}$ relativos ao sistema $\{O\}$.

$E_{l} \quad$ Origem do sistema de coordenadas $\left\{E_{l}\right\}$ fixo ao l-ésimo vértice da plataforma.

$\hat{F}_{i_{l}} \quad$ Vetor que representa a soma das forças e torque aplicados sobre o ligamento $i_{l}$.

$\hat{F}_{p} \quad$ Vetor que representa a soma das forças e torque aplicados sobre a plataforma.

$f_{i_{l}} \quad$ Força resultante, excluindo a força do atuador, exercida sob o centro de massa do ligamento $i_{l}$.

$f_{i_{l}}^{*} \quad$ Força de inércia exercida sobre o centro de massa do ligamento $i_{l}$.

$f_{p} \quad$ Força resultante exercida sobre o centro de massa da plataforma.

$f_{p}^{*} \quad$ Força de inércia exercida sobre o centro de massa da plataforma. 
$g \quad$ Aceleração da gravidade.

$G\left(\theta_{\mathbf{l}}\right)$ Vetor de torques de gravidade.

I Conjunto das juntas independentes.

I Matriz identidade.

i Subíndice utilizado para denotar uma junta ou ligamento de uma cadeia cinemática.

$i_{a} \quad$ Corrente de armadura do motor C.C.

$i_{e} \quad$ Corrente de excitação do motor C.C.

$I_{i_{l}}\left(t_{j}\right)$ Matriz de conversão.

$I_{\max _{i_{l}}}$ Corrente máxima de armadura do motor C.C. da junta $i_{l}$.

$I_{p} \quad$ Matriz de inércia da plataforma.

$I_{x x}, I_{y y}, I_{z z}$ Momentos de inércia de massa de um corpo rígido com relação a um sistema de referência fixo.

$I_{x y}, I_{y z}, I_{x z}$ Produtos de inércia de massa de um corpo rígido com relação a um sistema de referência fixo.

$\mathfrak{J}_{f} \quad$ Matriz Jacobiana de $f\left(\hat{T}^{k}\right)$.

$J_{A_{l}} \quad$ Matriz Jacobiana que relaciona as velocidades das juntas da perna $l$ com os graus de liberdade da garra relativa à perna $l$.

$J_{m} \quad$ Momento de inércia do rotor do motor C.C.

$J_{q_{l}} \quad$ Matriz Jacobiana que relaciona as velocidades das juntas da perna $l$ com as velocidades da plataforma.

$J_{q} \quad$ Matriz Jacobiana que relaciona as velocidades das juntas com as velocidades da plataforma.

$J_{x_{1_{l}}} \quad$ Matriz Jacobiana que relaciona as velocidades angulares da plataforma com as velocidades das juntas da perna $l$. 
$J_{x_{1}} \quad$ Matriz Jacobiana que relaciona as velocidades angulares da plataforma com as velocidades das juntas.

$J_{x_{2}} \quad$ Matriz Jacobiana que relaciona as velocidades angulares da plataforma com as velocidades angulares dos ângulos de Euler.

$k$ Superíndice utilizado para denotar uma iteração do processo recursivo de otimização.

$K_{b} \quad$ Constante do motor C.C.

$K_{e} \quad$ Constante de excitação do motor C.C.

$K_{m} \quad$ Constante de torque do motor.

$K_{p} \quad$ Constante de excitação magnética do motor C.C.

$\tilde{L} \quad$ Número de pernas fixas à superfície de apoio suportando o peso do robô.

L Número máximo de cadeias cinemáticas do sistema.

$l \quad$ Subíndice utilizado para denotar uma cadeia cinemática.

$L_{1} \quad$ Comprimento do vetor $\overrightarrow{E_{l} P}$.

$L_{2} \quad$ Comprimento do vetor $\overrightarrow{D_{l} E_{l}}$ da l-ésima perna.

$L_{3} \quad$ Comprimento do vetor $\vec{C}_{l} \overrightarrow{D_{l}}$ da l-ésima perna.

$L_{4} \quad$ Comprimento do vetor $\overrightarrow{B_{l} C_{l}}$ da l-ésima perna.

$L_{5} \quad$ Comprimento do vetor $\overrightarrow{A_{l} B_{l}}$ da l-ésima perna.

$L_{a} \quad$ Indutância de armadura do motor C.C.

M Número máximo de juntas de uma cadeia cinemática.

$M\left(\theta_{1}\right)$ Matriz de massa.

$m_{i_{l}} \quad$ Massa do ligamento $i_{l}$.

$m_{p} \quad$ Massa da plataforma. 
$N \quad$ Fator de discretização.

$n_{i_{l}} \quad$ Torque resultante, excluindo o torque do atuador, exercido em relação ao centro de massa do ligamento $i_{l}$.

$n_{i_{l}}^{*} \quad$ Torque de inércia exercido sobre o centro de massa do ligamento $i_{l}$.

$n_{p} \quad$ Torque resultante exercido sobre o centro de massa da plataforma.

$n_{p}^{*} \quad$ Torque de inércia exercido em relação ao centro de massa da plataforma.

$O \quad$ Origem do sistema de referencia $\{O\}$ fixo à superfície de apoio.

$\mathcal{P} \quad$ Conjunto das juntas passivas.

$\{P\}$ Sistema de coordenadas fixo ao centro da plataforma.

${ }^{O} P_{x},{ }^{O} P_{y},{ }^{O} P_{z}$ Componentes em $x, y$ e $z$ do ponto $P$ relativos ao sistema $\{O\}$.

$P \quad$ Origem do sistema de coordenadas $\left\{P_{l}\right\}$ fixo ao centro da plataforma.

$P(I)$ Posição do centro da plataforma na etapa I da andadura.

$P_{i_{l}} \quad$ Potência elétrica instantânea do motor C.C. da junta $i_{l}$.

$\vec{q} \quad$ Vetor formado por todas as variáveis das juntas do sistema.

${ }^{Y} R_{X}$ Matriz de rotação que descreve a orientação do sistema $X$ com respeito ao sistema $Y$.

$r \quad$ Factor de redução por engrenagem do motor C.C.

$R_{a} \quad$ Resistência de armadura do motor C.C.

$s \theta \quad$ Versão simplificada para representar o $\sin \theta$.

$\bar{t}_{\text {proc }}(\cdot)$ Tempo de processamento médio.

$\hat{\mathcal{T}} \quad$ Ponto de mínimo do vetor $\hat{T}$.

${ }^{Y} T_{X} \quad$ Matriz de transformação homogénea que descreve a transformação homogénea do sistema $X$ com respeito ao sistema $Y$. 
$t \theta \quad$ Versão simplificada para representar a $\tan \theta$.

$t_{a} \quad$ Tempo de um ciclo da andadura.

$t_{f} \quad$ Tempo de execução.

$t_{f}(\cdot)$ Tempo de execução do movimento da etapa $(\cdot)$.

$T_{i_{l}} \quad$ Vetor formado pelos valores discretos $\tau_{i_{l}}\left(t_{1}\right), \tau_{i_{l}}\left(t_{2}\right), \ldots, \tau_{i_{l}}\left(t_{N}\right)$

$t_{j} \quad$ Instante de tempo $j$

$t_{\text {proc }}$ Tempo de processamento total.

$t_{\text {proc }}(\cdot)$ Tempo de processamento da etapa $(\cdot)$.

$\bar{U}_{i_{l}}^{k} \quad$ Lei de controle discreta ótima para a junta $i_{l}$ na iteração $k$.

$U_{i_{l}} \quad$ Lei de controle discreta para a junta $i_{l}$.

$u_{i_{l}} \quad$ Lei de controle para o motor C.C. da junta $i_{l}$.

$\dot{v}_{c_{i_{l}}} \quad$ Velocidade do centro de massa do ligamento $i_{l}$.

$\vec{v}_{A_{l}} \quad$ Velocidade linear da garra relativa à perna $l$.

$\vec{v}_{E_{l}} \quad$ Velocidade linear do ponto $E_{l}$

$\vec{v}_{P} \quad$ Velocidade linear do centro da plataforma plataforma (ponto $P$ ).

${ }^{(i+1)_{l}} \dot{v}_{c_{i_{l}}}$ Velocidade do centro de massa do ligamento $i_{l}$ em relação ao sistema inercial $\{O\}$ expressa no sistema ${ }^{(i+1)_{l}}$.

${ }^{(i+1)_{l}} v_{i_{l}}$ Velocidade linear do ligamento $i_{l}$ em relação ao sistema inercial $\{O\}$, expressa no sistema de coordenadas do ligamento $\left\{(i+1)_{l}\right\}$.

$V_{a} \quad$ Tensão de armadura do motor C.C.

$V_{c} \quad$ Volumem de um corpo rígido.

$v_{i_{l}} \quad$ Velocidade linear do ligamento $i_{l}$.

$V_{\max _{i_{l}}}$ Tensão máxima de armadura do motor C.C. da junta $i_{l}$. 
$V_{m} \quad$ Tensão eletromotriz do motor C.C.

$\mathcal{W}_{A_{l}} \quad$ Espaço de trabalho da perna $l$.

$\mathcal{W}_{P_{l}} \quad$ Espaço de trabalho da plataforma relativo à perna $l$.

$\mathcal{W}_{P l_{\text {max }}}$ Espaço de trabalho máximo da plataforma relativo à perna $l$.

$\mathcal{W}_{P l_{\text {min }}}$ Espaço de trabalho mínimo da plataforma relativo à perna $l$.

$\mathcal{W}_{P} \quad$ Espaço de trabalho total da plataforma.

$\vec{x} \quad$ Vetor formado pelos graus de liberdade da plataforma.

$\vec{x}_{A_{l}} \quad$ Vetor que representa os graus de liberdade da garra relativa à perna $l$.

$\vec{x}_{i_{l}} \quad$ Vetor formado pelos graus de liberdade do ligamento $i_{l}$.

$x_{A E_{l}} \quad$ Coordenada $x$ relativa ao vetor ${ }^{E_{l}} \overrightarrow{E_{l}} A_{l}$.

$x_{m_{i_{l}}} \quad$ Vetor de estados do motor C.C. da junta $i_{l}$.

$x_{P A B_{l}}$ Coordenada em $x$ do vetor ${ }^{O} \overrightarrow{B_{l} P}$.

$y_{A E_{l}} \quad$ Coordenada $y$ relativa ao vetor ${ }^{E_{l}} \overrightarrow{E_{l}} \overrightarrow{A_{l}}$.

$y_{m_{i_{l}}} \quad$ Saída da equação de estados do motor C.C. da junta $i_{l}$.

$y_{P A B_{l}}$ Coordenada em $y$ do vetor ${ }^{O} \overrightarrow{B_{l} P}$.

${ }^{(i+1)_{l}} \hat{Z}_{(i+1)_{l}}$ Versor do eixo da junta expresso no sistema $\left\{(i+1)_{l}\right\}$.

$z_{A E_{l}} \quad$ Coordenada $z$ relativa ao vetor ${ }^{E_{l}} \overrightarrow{E_{l}} A_{l}$.

$z_{P A B_{l}}$ Coordenada em $z$ do vetor ${ }^{O} \overrightarrow{B_{l} P}$. 


\section{Sumário}

1 Introdução 1

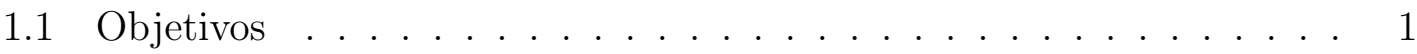

1.2 Motivação e estado da arte . . . . . . . . . . . . . . . . 1

1.3 Justificativa . . . . . . . . . . . . . . . . . . . . . . . . 8

1.4 Contribuições do trabalho . . . . . . . . . . . . . . . . . . 9

1.5 Estrutura do texto . . . . . . . . . . . . . . . . . . 9

2 Análise de Posição $\quad 12$

2.1 Introdução . . . . . . . . . . . . . . . . . . . . . . . . 12

2.2 Análise de posição direta . . . . . . . . . . . . . . . . . 13

2.2.1 Solução do problema direto para a plataforma . . . . . . . . . 14

2.2.2 Solução do problema direto para a perna . . . . . . . . . . . . 19

2.3 Análise de posição inversa . . . . . . . . . . . . . . . . 21

2.3.1 Solução do problema inverso para a plataforma . . . . . . . . 21

2.3.2 Solução do problema inverso para a perna . . . . . . . . . . . 27

3 Cinemática $\quad 31$

3.1 Introdução . . . . . . . . . . . . . . . . . . . . . . . 31

3.2 Análise cinemática de velocidade da plataforma . . . . . . . . . . . 32

3.2.1 Singularidade inversa da plataforma . . . . . . . . . . . 36 
3.2.2 Singularidade direta da plataforma . . . . . . . . . . . . 39

3.2.3 Singularidade combinada da plataforma . . . . . . . . . . 40

3.2.4 Aceleração da plataforma . . . . . . . . . . . . . . . 41

3.3 Análise cinemática de velocidade da garra . . . . . . . . . . . . . . 42

3.3.1 Singularidade da garra . . . . . . . . . . . . . . 42

3.3.2 Aceleração da garra . . . . . . . . . . . . . . . . . . 46

4 Dinâmica $\quad 48$

4.1 Introdução . . . . . . . . . . . . . . . . . . . . . . . . . 48

4.2 Acelerações dos ligamentos . . . . . . . . . . . . . . . . . 51

4.2.1 Aceleração angular . . . . . . . . . . . . . . . . . 51

4.2.2 Aceleração linear . . . . . . . . . . . . . . . . . . . . . 52

4.3 Matriz de inércia de cada ligamento . . . . . . . . . . . . . . . 52

4.4 Análise dinâmica da plataforma . . . . . . . . . . . . . . 54

4.5 Análise dinâmica da perna . . . . . . . . . . . . . . . . . 56

4.6 Efeitos não modelados . . . . . . . . . . . . . . . . . . . . . 59

5 Algoritmo de Otimização $\quad 61$

5.1 Introdução . . . . . . . . . . . . . . . . . . . . . 61

5.2 Modelo do motor . . . . . . . . . . . . . . . . . . 62

5.2.1 Controle independente por junta . . . . . . . . . . . . 65

5.2 .2 Discretização . . . . . . . . . . . . . . . . . 68

5.3 Apresentação do algoritmo . . . . . . . . . . . . . . . . 69

5.4 Análise da convergência do algoritmo . . . . . . . . . . . . . . . . . 71

$\begin{array}{lll}6 & \text { Resultados numéricos } & 78\end{array}$

6.1 Introdução . . . . . . . . . . . . . . . . . . . . 78 
6.2 Padrão de movimentos ou andadura do robô . . . . . . . . . . . . . 78

6.3 Classificação das juntas . . . . . . . . . . . . . . . . 80

6.4 Condições prévias . . . . . . . . . . . . . . . . . 82

6.5 Resultados Finais . . . . . . . . . . . . . . . . . . 85

$\begin{array}{llr}7 & \text { Conclusões } & 99\end{array}$

A Velocidades angulares e lineares dos ligamentos $\quad 102$

B Acelerações angulares e lineares dos ligamentos 105

C Variáveis dependentes na etapa I da andadura do robô 112

D Motor RS-540-12v 115

E Kit universal de redução por engrenagem Tamiya $70103 \quad 116$

$\begin{array}{lll}\text { F } & \text { Bateria selada BT-12M14AC } & 117\end{array}$

G Sequência de movimentos do robô $\quad 119$ 


\section{Capítulo 1}

\section{Introdução}

\subsection{Objetivos}

Os objetivos deste trabalho são a modelagem de um robô quadrúpede e o desenvolvimento de um algoritmo para a otimização das perdas de energia elétrica associadas a sua locomoção. Como protótipo dos testes foi escolhido o robô Kamambaré ${ }^{1}$ em fase de projeto pelo Laboratório de Automação e Controle (LAC) da Escola Politécnica da Universidade de São Paulo.

\subsection{Motivação e estado da arte}

A palavra robô é uma derivação da palavra tcheca "robota" introduzida em 1920 na peça de teatro "Robôs Universais de Rossum" escrita pelo dramaturgo tcheco Karel Capek. Na peça os robôs eram descritos como máquinas semelhantes aos humanos, mas que trabalhavam de forma incansável. Desde então a palavra robô formou parte de nosso vocabulário. Hoje em dia, os robôs podem ser descritos como máquinas que operam com algum grau de autonomia, controlados geralmente por um computador, um processador ou a interface humana.

O desenvolvimento da robótica pode teve inicio depois da Segunda Grande

\footnotetext{
${ }^{1}$ Kamambaré é a palavra utilizada em lingua Tupi-Guarani para camaleão.
} 
Guerra. Os primeiros manipuladores robóticos foram desenvolvidos pelos National Laboratories em Oak Ridge e Argonne nos Estados Unidos, com a finalidade de manipular material radioativo. Este braço robótico ou manipulador era controlado através de ligamentos mecânicos que podiam ser acionados por motores elétricos ou hidráulicos. Na época, estes equipamentos foram chamados de tele-operadores.

Em 1961 uma companhia chamada Unimation instalou seu primeiro robô na General Motors (Figura 1.1). O grande diferencial deste robô era que ele podia ser reprogramado com relativa facilidade. Desta forma, podia executar uma grande quantidade de tarefas diferentes. A partir deste momento os robôs começaram a ser instalados na indústria, principalmente em linhas de montagem de automóveis.

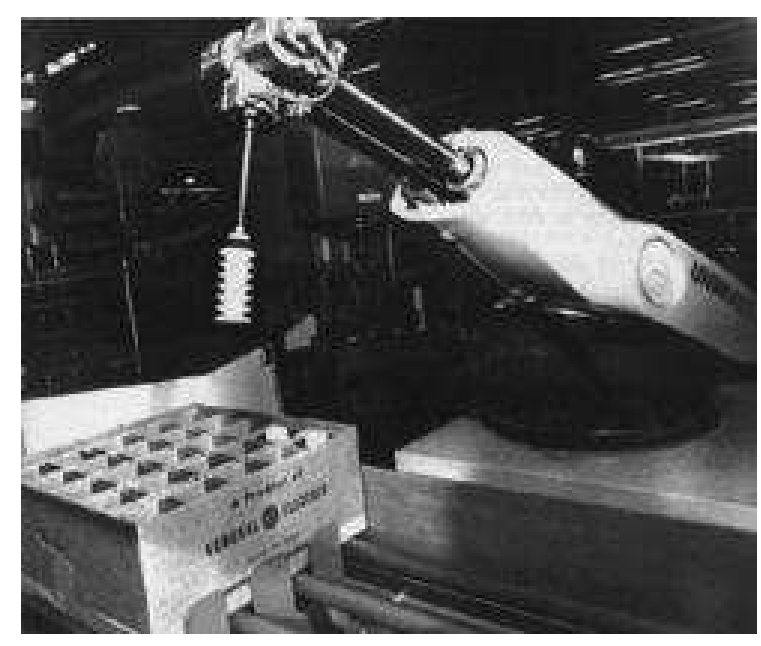

Figura 1.1: Primeiro robô da Unimation construído no ano 1961. Fonte: RobotWorx (2011)

Paralelamente ao desenvolvimento dos manipuladores, foi sendo desenvolvida outra área de pesquisa dentro da ciência da robótica. Motivada pelo reduzido espaço de trabalho de um manipulador industrial e pelo desenvolvimento de novas tecnologias, pouco a pouco foram surgindo os primeiros robôs móveis.

Os robôs móveis podem ser autônomos ou não. Entenda-se por autônomos aqueles que podem executar alguma tarefa pre-determinada sem a supervisão humana. Em qualquer uma das duas situações o salto tecnológico foi grande. 
A maioria dos robôs móveis atuais se movimenta com rodas. Este fato é devido à maior eficiência que apresenta esta locomoção em ambientes humanos onde a maioria das superficies são regulares (Luk et al., 2005). Não obstante, a eficiência dos robôs guiados por rodas cai consideravelmente em ambientes de superfícies irregulares. A solução foi então a construção de robôs movidos por pernas, utilizando outros tipos de locomoção inspiradas nas formas biológicas (Pfeiffer et al., 1995a,b; Espenschied et al., 1996; Siegwart e Nourbakhsh, 2004; Haynes e Rizzi, 2006; Ingvast, 2006).

Os pioneiros no desenvolvimento de robôs móveis com pernas foram os japoneses. O Instituto Tecnológico de Tokyo (ITT) começou a desenvolver robôs quadrúpedes desde o ano de 1976 com seu primeiro protótipo chamado KUMO-I. A partir deste momento e com o desenvolvimento da indústria eletrônica digital e dos novos componentes o ITT desenvolveu muitos protótipos de robôs quadrúpedes sendo os mais conhecidos sua familia de robôs chamada TITAN (Figura 1.2), (Hirose e Kato, 2000).

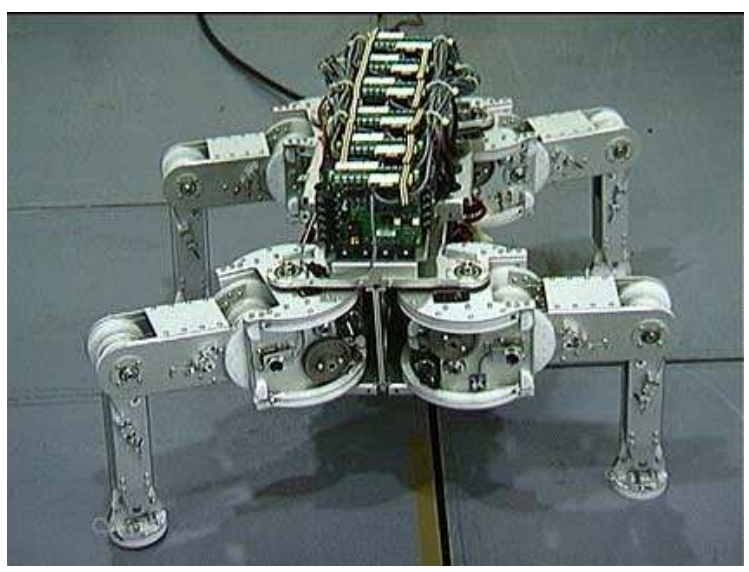

Figura 1.2: Robô quadrúpede TITAN VIII desenvolvido pelo ITT. Fonte: Robotics Lab (2011)

A locomoção por pernas é caracterizada pelo conjunto de pontos de contato que se encontram entre o robô e a superfície de agarramento. As principais vantagens destes sistemas consistem na sua facilidade de adaptação e manobra em terrenos ir- 
regulares, já que somente é preciso ter um número finito de pontos de contato com a superfície e não interessa o que exista entre estes pontos. Outro ponto interessante é que estes robôs podem ultrapassar obstáculos, subir ou descer superfícies irregulares e atravessar buracos sempre que a largura destes não seja muito grande. Na literatura existe reportada uma grande quantidade de robôs com pernas (Abderrahim et al., 1999; Choi et al., 2000; Brown e Huissoon, 2000; Galvez et al., 2001; Armada et al., 2003; Weingartent et al., 2004; Tokhi et al., 2005; Choi e Park, 2006), tendo alguns deles até a capacidade de escalar superfícies verticais como por exemplo, a serie de robôs RiSE desenvolvidos pelo laboratório Kod*Lab da Universidade de Pensilvânia. (Figuras 1.3 e 1.4).

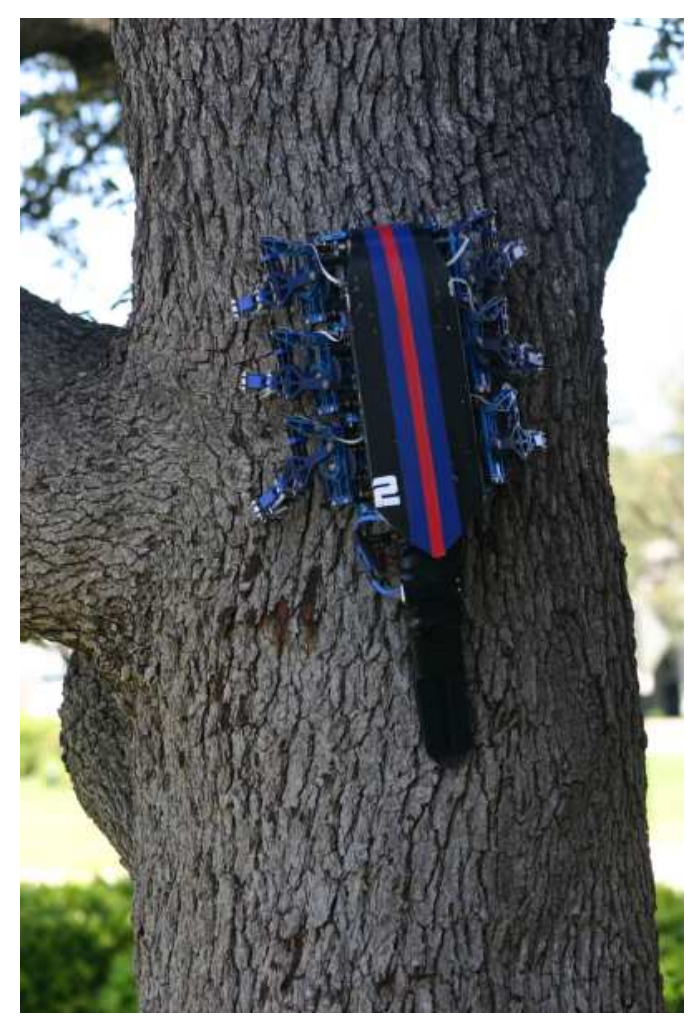

Figura 1.3: Robô RiSE versão 1.5 Fonte: Kod $*$ Lab (2011)

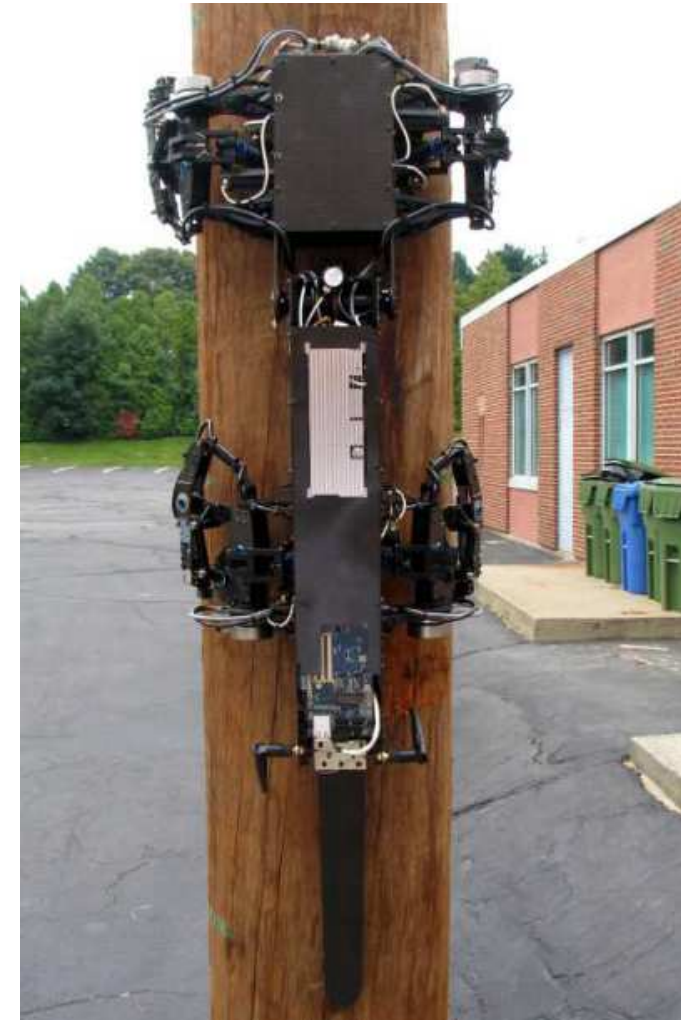

Figura 1.4: Robô RiSE versão 3: Fonte: Haynes et al. (2009).

Logicamente a principal desvantagem destes robôs é sua maior complexidade mecânica e de controle. Por exemplo, cada perna do robô geralmente tem vários 
graus de liberdade que devem ser controlados de forma sincronizada. Ao mesmo tempo a perna tem de sustentar parte do peso do robô e ser capaz de movê-lo. Além disto existem problemas de instabilidade e de desequilíbrio que não estavam presentes nos robôs guiados por rodas.

A maioria dos robôs com pernas são controlados por motores elétricos devido à simplicidade e ao pouco custo desta técnica, porém a dificuldade com os motores elétricos está em que estes alcançam seu maior torque a altas velocidades, razão pela qual é preciso utilizar mecanismos redutores. As engrenagens redutoras introduzem problemas de atrito, de folga e aumentam o custo de fabricação dos robôs. Devido a estes problemas e ao fato que na maioria das vezes o robô móvel carrega sua própria fonte de energia, normalmente uma bateria elétrica de potência finita, é que surge a idéia de utilizar um algoritmo de controle na otimização das perdas de energia elétrica em seus atuadores.

A aplicação de um controle ótimo na robótica é um problema amplamente tratado na literatura, porém, sob outros pontos de vista. O chamado Optimal Free Motion Planning Problem (OFMPP) foi analisado pela primeira vez em 1971 por Kahn e Roth e desde então tem sido uma área de grande interesse e motivação para a comunidade científica internacional. O OFMPP pode ser visto como um problema de controle ótimo clássico. Sua resolução é comumente realizada utilizando métodos numéricos que podem ser classificados em duas categorias, a direta e a indireta (Stryk e Bulirsch, 1992; Hull, 1997; Betts, 1998). Os métodos indiretos são baseados no cálculo das variações ou Princípio do Máximo de Pontryagin (Bryson e Ho, 1975). Eles conduzem geralmente a problemas de múltiplos valores de contorno que devem ser resolvidos usando técnicas tais como o método de múltiplos disparos, e precisam de um bom ponto de partida. Infelizmente, para a solução de tais métodos é preciso recorrer a algoritmos de cálculos numéricos geralmente muito difíceis de implementar e que consumem uma grande quantidade de tempo ao serem executados. Por último, o usuário deve ter um profundo conhecimento sobre a natureza física e matemática do problema de otimização a resolver. 
Para superar estes inconvenientes foram propostos posteriormente os métodos diretos. Estes são baseados na transformação do problema original em um problema de programação não-linear (PNL) pela discretização de algumas ou de todas as variáveis do sistema dinâmico (estados, controles, etc). Esses métodos provaram ser robustos, simples, e requerem uma mínima interação do usuário para definir o problema de otimização (Chettibi et al., 2005).

A aplicação de tais métodos em robôs com pernas é relativamente recente, o que pode ser devido aos seguintes fatores:

- A complexidade do seu movimento.

- Dificuldade para escolher a aproximação inicial da solução.

- Problemas de planejamento de trajetórias.

- Dificuldade de convergência.

- Dificuldades para conseguir o modelo dinâmico de sua estrutura quando comparados com os manipuladores industriais.

- Existência de múltiplas soluções.

A topologia de um robô com pernas varia constantemente em função do padrão de movimento ou andadura do robô. Isto significa que estes sistemas têm presentes cadeias fechadas que abrem quando as pernas do robô se separam da superfície de apoio e que se fecham quando estas encontram um novo ponto de apoio. Esta topologia complexa implica em um maior número de restrições e aumento das singularidades mecânicas como relatado em Angeles (2007).

Existem relatados na literatura alguns exemplos que mostram a aplicação de tais métodos, aplicados a robôs paralelos ou seriais, encontram-se assim técnicas de controle para otimizar a trajetória de manipuladores minimizando determinados parâmetros físicos tais como, energia mecânica, esforço de controle, jerk ou tempo 
(Facchinei, 1997; Guo e Zhang, 2001; Bobrow et al., 2004; Luo et al., 2004; Chettibi et al., 2005), porém o estudo das perdas elétricas como índice de otimização nunca foi tratado. Esta análise das perdas elétricas permite maximizar o tempo de uso da bateria, o que é muito importante em aplicações onde é preciso uma grande autonomia do robô como é o caso, por exemplo, de pesquisas biológicas na floresta, objetivo inicial do Kamambaré.

Neste trabalho será apresentado um algoritmo de controle ótimo baseado na minimização das perdas de energia elétrica decorrentes do movimento mecânico de cadeias cinemáticas fechadas ou abertas. Para isto as juntas do mecanismo serão divididas em conjuntos sobre os quais se aplicarão diferentes leis de controle geradas pelo próprio algoritmo. Como o método proposto se baseia no modelo mecânico do sistema é necessário contar com um bom modelo físico-matemático do sistema. Visando este objetivo será levantado o modelo do robô Kamambaré (Figura 1.5) em fase de projeto pelo Laboratório de Automação e Controle da Universidade de São Paulo e em seguida será aplicado o algoritmo mencionado acima no controle da andadura do mesmo.

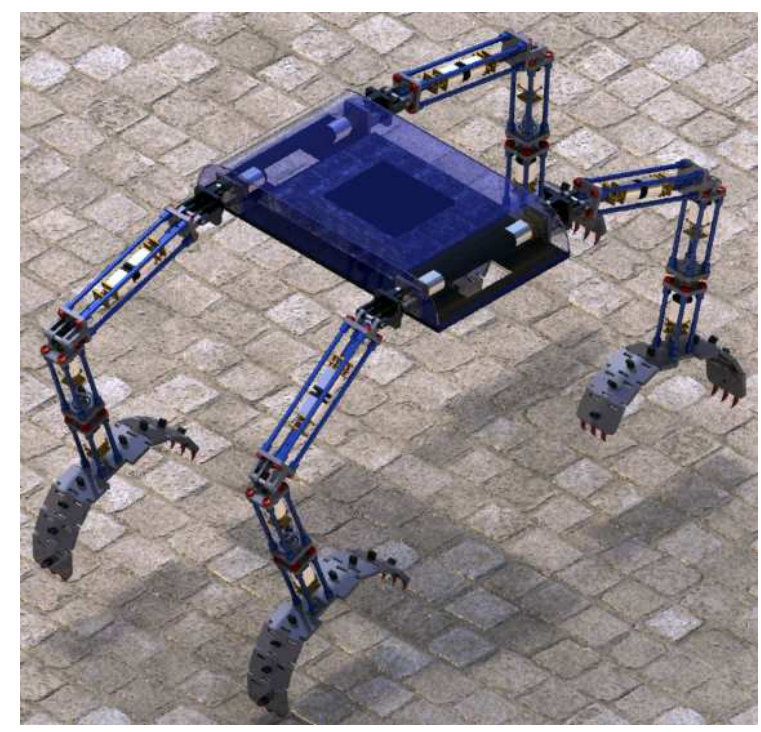

Figura 1.5: Robô Kamambaré 


\subsection{Justificativa}

O mercado da robótica é um mercado que está se expandindo de forma acelerada. Há apenas alguns anos os robôs saíram das indústrias e hoje já fazem parte da nossa rotina diária. A Figura 1.6 mostra as tendências do mercado da robótica desde a atualidade até os próximos anos. Pode-se observar que o maior crescimento não é mais na indústria e sim nas áreas de serviços, médicas e biológicas. Especificamente nestas áreas é onde existe um maior desenvolvimento de robôs móveis e justamente, é nestes tipos de robôs, que o consumo de energia pode ser um parâmetro crítico de projeto.

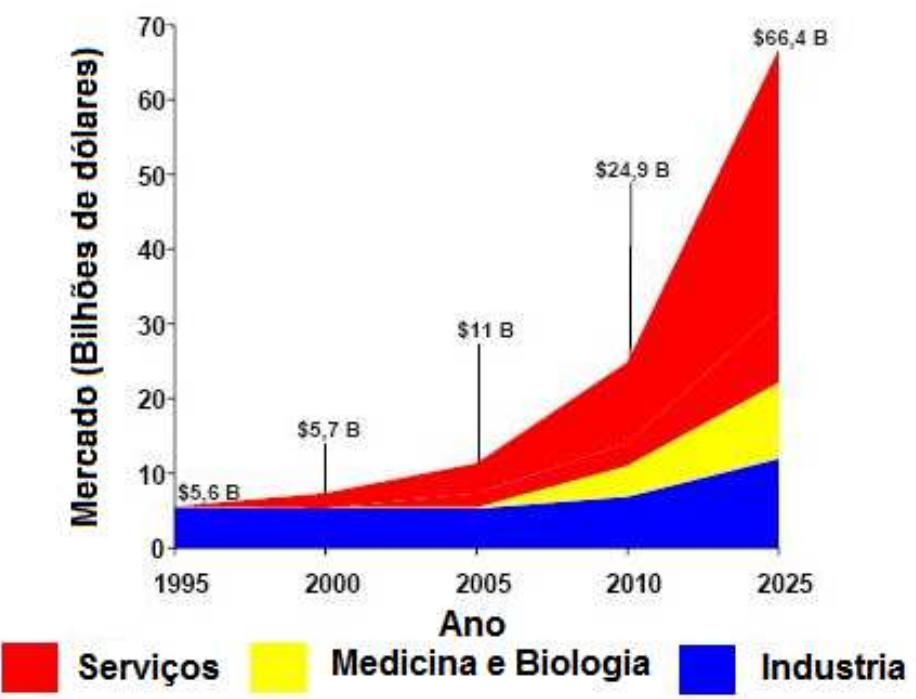

Figura 1.6: Comportamento do mercado da robótica de 1995 até o ano 2025. Fonte: EurAtiv.com (2005)

Paralelamente ao crescimento na indústria da robótica, uma outra justificativa é que até o momento não foram encontradas técnicas de otimização das perdas elétricas para o controle de cadeias cinemáticas fechadas ou abertas. A maioria dos trabalhos consultados trata o tema da otimização energética sob o ponto de vista mecânico para trajetórias em manipuladores industriais. Esta característica é fácil de entender devido ao fato de que estes manipuladores são conectados diretamente à 
rede elétrica e parâmetros como o tempo de execução de uma determinada tarefa tem maior importância do que o consumo elétrico. Além disto, as técnicas de redução de consumo elétrico podem ser aplicadas também a manipuladores que trabalhem em sistemas com energia limitada, como é o caso do espaço cósmico, em sistema de exploração ou em robôs subaquáticos.

\subsection{Contribuições do trabalho}

As contribuições deste trabalho podem ser divididas em dois grandes grupos. No primeiro, situam-se aquelas relativas à modelagem do robô Kamambaré. Neste sentido, tem-se a obtenção e a análise dos modelos cinemáticos e dinâmicos das

pernas e da plataforma como é relatado em Bernardi et al. (2009); Potts e Da Cruz (2010, 2011b).

No outro grupo de contribuições encontram-se, o desenvolvimento e aplicação de um novo algoritmo de minimização das perdas elétricas nos motores que controlam as juntas de sistemas mecatrônicos. Logo, pertencem a este grupo a formulação, demonstração e aplicação do algoritmo proposto no modelo do Kamambaré como é apresentado em Potts et al. (2008b,a, 2009); Potts e Da Cruz (2011a).

Todas as contribuições e resultados antes mencionados foram divulgados à comunidade acadêmica em congressos nacionais e internacionais, foi publicado um capítulo de livro e um último artigo para periódico, decorrente deste trabalho, está em fase de conclusão.

\subsection{Estrutura do texto}

Para melhor situar o leitor no que se refere à estrutura deste trabalho será feita uma breve apresentação do conteúdo do mesmo.

O primeiro capítulo é uma breve introdução das motivações que levaram o 
autor ao desenvolvimento desta pesquisa. Além disto, trata-se dos objetivos pretendidos, da história e estado da arte do assunto e justifica-se a importância do tema escolhido.

Após a introdução o trabalho encontra-se dividido em duas partes, a primeira parte descreve a modelagem do robô e a segunda trata da aplicação do algoritmo de controle ótimo no modelo obtido e seus resultados. Ambas as partes estão divididas em capítulos, mantendo-se assim o mesmo padrão em todo o trabalho. A modelagem do robô é mostrada sempre em duas variantes: primeiramente se apresenta o modelo da plataforma do robô quando existe pelo menos uma cadeia cinemática fechada formada por dois ou mais pernas e, em seguida, é mostrado o modelo da perna livre com base na plataforma.

Diante do exposto, o trabalho será apresentado de acordo com a seguinte estrutura:

Parte 1:

No capitulo 2 é feita uma apresentação geral do robô Kamambaré e são resolvidos os problemas cinemáticos de posição inversa e direta da plataforma e das pernas do robô.

No capítulo 3 é descrita uma análise cinemática de velocidade sob o ponto de vista do Jacobiano do sistema. Além disto são analisadas as condições de singularidade que se apresentam para determinadas configurações de posição e projeto.

O capítulo 4 apresenta uma análise da dinâmica do robô. O método utilizado para tal foi o do Princípio do Trabalho Virtual ou d'Alembert para o modelo dinâmico da plataforma e o método iterativo de Newton-Euler para o caso das pernas no ar.

Parte 2:

No capítulo 5 é apresentado o algoritmo de controle ótimo proposto para a otimização das perdas de energia elétrica na locomoção do robô Kamambaré. Neste 
capitulo é feito um desenvolvimento matemático das idéias que levaram o autor à elaboração do algoritmo e ainda é feita uma demonstração da convergência do mesmo.

Já no capítulo 6 são apresentados e discutidos os resultados numéricos obtidos no controle ótimo da andadura do robô.

Finalmente serão apresentadas as conclusões do trabalho e as recomendações para desenvolvimentos futuros. 


\section{Capítulo 2}

\section{Análise de Posição}

\subsection{Introdução}

Kamambaré é uma plataforma robótica bio-inspirada (Figura 2.1), desenhada considerando habilidades tais como a locomoção em ambientes irregulares, o contorno de obstáculos, e o deslocamento vertical sobre diferentes tipos de superfícies. Sua estrutura mecânica consiste de um corpo rígido central com quatro pernas idênticas e simetricamente distribuídas. Cada perna é composta por 4 ligamentos conectados por meio de 3 juntas rotacionais e está ligada ao corpo central através de uma quarta junta rotacional. Cada junta possui somente um grau de liberdade (GDL). Ao final de cada perna encontra-se uma garra controlada por um quinto motor elétrico (Bernardi e Da Cruz, 2007; Bernardi et al., 2009).

A topologia do Kamambaré, como a de todos os robôs móveis com pernas, é variante no tempo. Resultado de seu próprio deslocamento, teremos dois problemas diferentes a resolver. Primeiramente, quando exista pelo menos uma cadeia cinemática fechada entre a superfície de apoio e a plataforma, formada pelas pernas que suportam o peso do robô, o comportamento deste será similar ao de um robô paralelo. Por outro lado, se uma perna do robô se encontra no ar à procura de um novo ponto de agarramento, o modelo que a melhor descreve é o de uma cadeia cinemática aberta, modelo este similar ao de um manipulador industrial serial. Através deste 


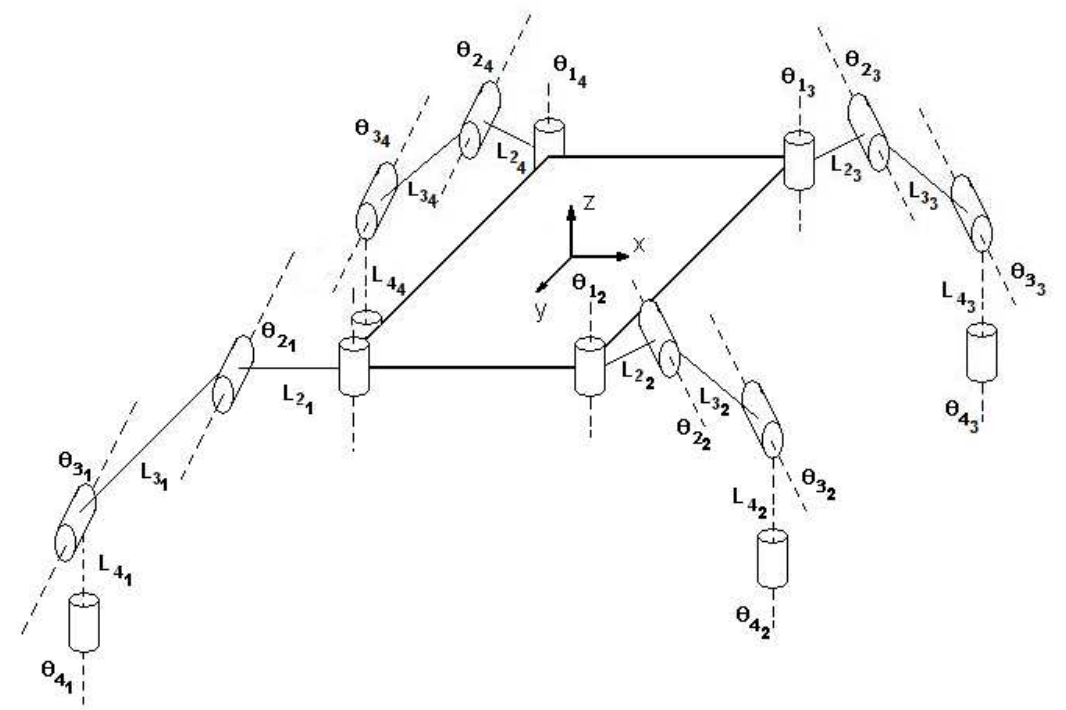

Figura 2.1: Projeto mecânico do robô Kamambaré.

trabalho nos referiremos a estas duas topologias como modelo da plataforma para o caso da modelagem paralela e modelo da perna para o segundo caso comentado.

Um aspecto interessante a destacar são os graus de liberdade do robô. Os graus de liberdade de um mecanismo ou GDL, são a quantidade de parâmetros independentes ou entradas necessárias para especificar a configuração do mecanismo completamente. Neste sentido para o caso da plataforma o robô terá 4 graus de liberdade, três de posição e um de rotação em torno ao eixo $Z$ perpendicular à plataforma. No caso da perna e especificamente a garra (efetuador), ela terá 4 graus de liberdade, três de posição e um de rotação em torno de seu eixo $Z$.

\subsection{Análise de posição direta}

Antes de o robô adotar uma nova posição, primeiramente deve ser calculada com exatidão a posição de seu centro de massa. Este problema, que às vezes chega a ser bastante complexo, é chamado por alguns autores como problema de análise de posição ou problema cinemático de posição (Tsai, 1999). Existem dois tipos de problemas de análise de posição: o direto e o inverso. 
No caso do problema da análise de posição direta, os valores dos ângulos das juntas são dados e o problema é encontrar as coordenadas do centro do corpo ou garra do robô.

\subsubsection{Solução do problema direto para a plataforma}

Comecemos nossa análise pela modelagem da plataforma do Kamambaré quando pelo menos duas pernas estão presas à superfície de apoio. Aqui o comportamento do robô é o de um robô paralelo, devido à existência de uma cadeia cinemática fechada.

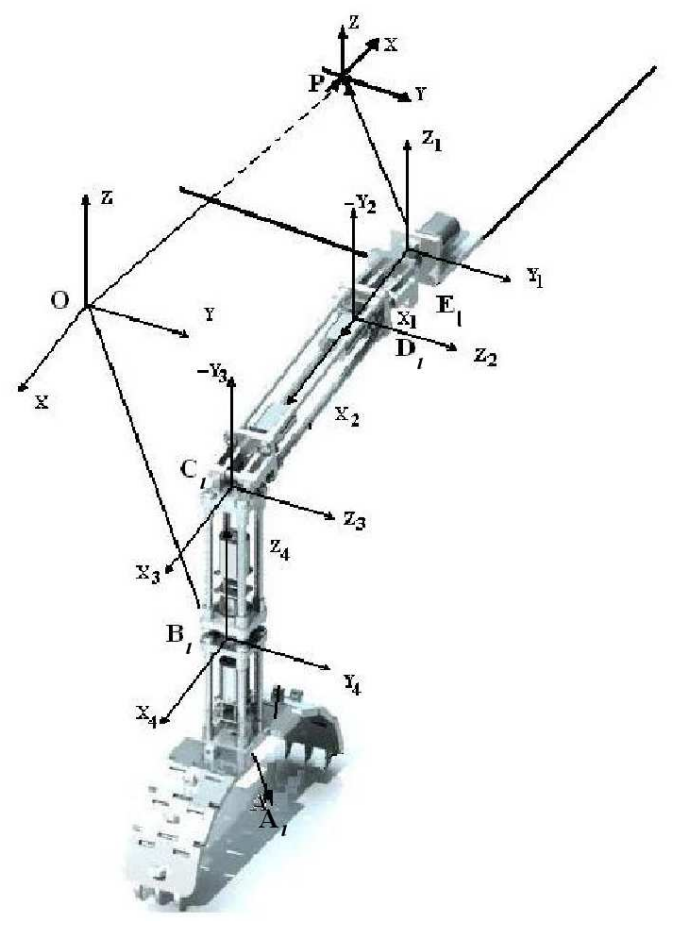

Figura 2.2: Perna do Kamanbaré

Na Figura 2.2, os sistemas dos ligamentos foram escolhidos conforme a convenção de Denavit-Hartenberg. Assim, é possível formular a seguinte equação vetorial para a perna $l$ do robô Kamambaré $(l=1, \ldots, 4)$ :

$$
{ }^{O} \overrightarrow{O A_{l}}+{ }^{O} \overrightarrow{A_{l} B_{l}}+{ }^{O} \overrightarrow{B_{l} C_{l}}+{ }^{O} C_{l} \overrightarrow{D_{l}}+{ }^{O} \overrightarrow{D_{l} E_{l}}+{ }^{O} \overrightarrow{E_{l} P}={ }^{O} \overrightarrow{O P}
$$




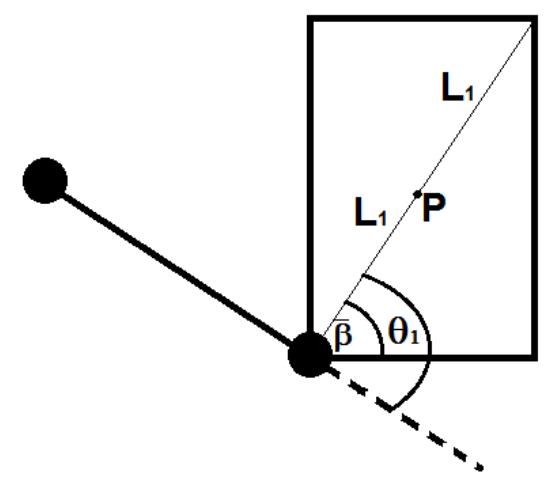

Figura 2.3: Definição do ângulo $\theta_{1_{l}}$

Esta equação pode ser entendida como: a partir do ponto $O$, origem do sistema de coordenadas $\{O\}$ fixo na superfície de apoio, qualquer perna presa à superfície tem que satisfazer a equação 2.1. Os pontos ${ }^{O} B_{l},{ }^{O} C_{l},{ }^{O} D_{l}$ são as origens dos sistemas relativos ao sistema $\{O\}$, fixos aos ligamentos 4,3 e 2 respectivamente. Os pontos ${ }^{O} E_{l}$ e $P$ denotam a origem dos sistemas $\left\{E_{l}\right\}$ e $\{P\}$ fixos ao l-ésimo vértice da plataforma e ao centro da mesma respectivamente. Os sub-índices $i$ e $l$ serão utilizados para representar as juntas e pernas do robô respectivamente, porém a notação utilizada com mais frequência será $i_{l}$, a qual representa a i-ésima junta $(i=1,2,3,4)$ da l-ésima perna $(l=1,2,3,4)$ do robô. Os ângulos $\theta_{1_{l}}$ serão definidos como aparece na Figura 2.3, onde o valor de $\bar{\beta}$ depende da geometria da plataforma.

A partir do ponto ${ }^{O} B_{l}$ e avançando pela perna no sentido da plataforma temos uma sucessão de transformações até o ponto de união da perna com a plataforma. Os ângulos de rotação em torno do eixo $Z_{i_{l}}$ de cada junta numa perna foram definidos começando pelo ponto ${ }^{O} E_{l}$, como $\theta_{1_{l}}, \theta_{2_{l}}, \theta_{3_{l}}$ e $\theta_{4_{l}}$. Assim cada perna do robô pode ser considerada como um manipulador serial onde a garra atua como base do manipulador e a plataforma como seu efetuador, ou vice-versa. Num sistema como este, as equações de translação e rotação de cada perna podem ser obtidas utilizando a parametrização de Denavit-Hartenberg. Este método é baseado nas rotações dos sistemas de referências consecutivos de cada junta (Craig, 1989). Utilizando este 
método e colocando a primeira junta no ponto $B_{l}$ da Figura 2.2 obtém-se a tabela 2.1:

Tabela 2.1: Tabela de Denavit-Hartenberg para a solução do problema direto.

\begin{tabular}{ccccc}
\hline$i$ & $\alpha_{i-1}$ & $a_{i-1}$ & $d_{i}$ & $\theta_{i_{l}}$ \\
\hline 4 & 0 & 0 & $L_{4}$ & $\theta_{4_{l}}$ \\
3 & $\frac{\pi}{2}$ & 0 & 0 & $\theta_{3_{l}}$ \\
2 & 0 & $L_{3}$ & 0 & $\theta_{2_{l}}$ \\
1 & $-\frac{\pi}{2}$ & $L_{2}$ & 0 & $\theta_{1_{l}}$ \\
& 0 & $L_{1}$ & 0 & 0 \\
\hline
\end{tabular}

Nesta tabela, $\alpha_{i-1}$ representa o ângulo entre os eixos $Z_{i-1}$ e $Z_{i}$ em torno do eixo $X_{i-1}$ de duas juntas consecutivas e $a_{i-1}$ a distância entre estes eixos segundo $X_{i-1}$ também. De forma similar $d_{i}$ é a distância de $X_{i-1}$ a $X_{i}$ medida segundo $Z_{i}$; por fim, $\theta_{i}$ é o ângulo entre $X_{i-1}$ e $X_{i}$ medido em torno de $Z_{i}$.

Os comprimentos $L_{5}, L_{4}, L_{3}, L_{2}$ e $L_{1}$ correspondem aos comprimentos dos vetores ${ }^{O} \vec{A}_{l} B_{l},{ }^{O} \overrightarrow{B_{l} C_{l}},{ }^{O} C_{l} \overrightarrow{D_{l}},{ }^{O} \overrightarrow{D_{l} E_{l}}$ e ${ }^{O} \overrightarrow{E_{l} P}$ respectivamente.

Uma vez construída a Tabela 2.1 podem-se obter as matrizes de transformação homogênea para o sistema através da equação de Denavit-Hartenberg. Começando no sistema $\{O\}$ e avançando até o centro da plataforma (ponto $P$ ) tem-se a seguinte transformação:

$$
{ }^{O} T_{P}={ }^{O} T_{A_{l}} \cdot{ }^{A_{l}} T_{B_{l}} \cdot{ }^{B_{l}} T_{C_{l}} \cdot{ }^{C_{l}} T_{D_{l}} \cdot{ }^{D_{l}} T_{E_{l}} \cdot{ }^{E_{l}} T_{P}
$$

onde ${ }^{O} T_{P}$ representa a transformação homogênea entre os sistemas de coordenadas $\{O\}$ e $\{P\}$ sendo,

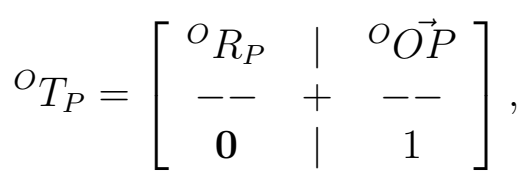




$$
\begin{aligned}
{ }^{O} T_{A_{l}} & =\left[\begin{array}{ccc}
{ }^{O} R_{A_{l}} & \mid & { }^{O} \overrightarrow{O A_{l}} \\
-- & + & -- \\
0 & \mid & 1
\end{array}\right], \\
{ }^{A} T_{B_{l}} & =\left[\begin{array}{ccc}
{ }^{A_{l}} R_{B_{l}} & \mid & { }^{O} \overrightarrow{A_{l} B_{l}} \\
-- & + & -- \\
0 & \mid & 1
\end{array}\right]
\end{aligned}
$$

$\mathrm{e}$

$$
{ }^{B_{l}} T_{P}=\left[\begin{array}{ccc}
{ }^{B_{l}} R_{P} & \mid & { }^{O} \overrightarrow{B_{l} P} \\
-- & + & -- \\
0 & \mid & 1
\end{array}\right] \text {. }
$$

De acordo com a matriz de transformação homogênea (2.3), a posição do ponto $P$ relativa ao sistema $\{O\}$ é dada pelos três primeiros elementos da última coluna de $(2.3)$ e a rotação relativa do vetor $\overrightarrow{E_{l} P}$ com relação ao sistema da base $\{O\}$ é dada pela sub-matriz ${ }^{O} R_{P}$ de (2.3). Assume-se também, que as matrizes de rotação ${ }^{O} R_{A_{l}}$ e ${ }^{A_{l}} R_{B_{l}}$ são iguais à matriz identidade e que o vetor ${ }^{O} \vec{A}_{l} B_{l}$ é sempre ortogonal à superfície de agarramento.

Logo,

$$
o_{P}=\left[\begin{array}{ccc}
c \theta_{4_{l}} c \theta_{2_{2} 3_{l}} c \theta_{1_{l}}+s \theta_{4_{l}} s \theta_{1_{l}} & -c \theta_{4_{l}} c \theta_{2_{3} 3_{l}} s \theta_{1_{l}}+s \theta_{4_{l}} c \theta_{1_{l}} & c \theta_{4_{l}} s \theta_{2_{l} 3_{l}} \\
s \theta_{4_{l}} c \theta_{2_{l} 3_{l}} c \theta_{1_{l}}-c \theta_{4_{l}} s \theta_{1_{l}} & -s \theta_{4_{l}} c \theta_{2_{l} 3_{l}} s \theta_{1_{l}}-c \theta_{4_{l}} c \theta_{1_{l}} & s \theta_{4_{l}} s \theta_{2_{l} 3_{l}} \\
s \theta_{2_{l} 3_{l}} c \theta_{1_{l}} & -s \theta_{2_{l} 3_{l}} s \theta_{1_{l}} & -c \theta_{2_{l} 3_{l}}
\end{array}\right]
$$

e a posição do ponto $P$ expressa no sistema $\{O\}$, relativa à perna $l$ é dada por:

$$
{ }^{O} \overrightarrow{O P}={ }^{O} \overrightarrow{O A_{l}}+{ }^{O} \overrightarrow{A_{l} B_{l}}+{ }^{O} \overrightarrow{B_{l} P}
$$

onde

$$
{ }^{o} \overrightarrow{B_{l} P}=\left[\begin{array}{c}
\left(c \theta_{4_{l}} c \theta_{2_{l} 3_{l}} c \theta_{1_{l}}+s \theta_{4_{l}} s \theta_{1_{l}}\right) L_{1}+c \theta_{4_{l}}\left(c \theta_{2_{l} 3_{l}} L_{2}+c \theta_{3_{l}} L_{3}\right) \\
\left(s \theta_{4_{l}} c \theta_{2_{l} 3_{l}} c \theta_{1_{l}}-c \theta_{4_{l}} s \theta_{1_{l}}\right) L_{1}+s \theta_{4_{l}}\left(c \theta_{2_{l} 3_{3}} L_{2}+c \theta_{3_{l}} L_{3}\right) \\
s \theta_{2_{l} 3_{l}} c \theta_{1_{l}} L_{1}+s \theta_{2_{l} 3_{l}} L_{2}+s \theta_{3_{l}} L_{3}+L_{4}
\end{array}\right]
$$

No texto, serão utilizadas as notações $c \theta, s \theta$ e $t \theta$ para as representações $\cos \theta$, $\sin \theta$ e $\tan \theta$ respectivamente e $\theta_{x_{l} y_{l}}$ será tratado como $\theta_{x_{l}}+\theta_{y_{l}}$. 
Por fim, a partir dos valores dos ângulos $\theta_{1_{l}}, \theta_{2_{l}}, \theta_{3_{l}}$ e $\theta_{4_{l}}$ e da posição do ponto $A_{l}$ pode ser deduzida a posição do centro da plataforma. Esta equação é válida para qualquer perna do robô Kamambaré, isto é, para $l=1, \ldots, 4$.

Com relação à orientação da plataforma ela é dada pela matriz de rotação ${ }^{B_{l}} R_{P}$ porém, a orientação da plataforma pode ser entendida também como o deslocamento angular entre os sistemas de coordenadas $\{O\}$ e $\{P\}$.

Uma forma simples de estabelecer este deslocamento é por meio dos ângulos de Euler. A matriz dos ângulos de Euler é baseada em três rotações sucessivas. Estas três rotações podem ser representadas como a pós-multiplicação de três matrizes de rotação:

$$
{ }^{o} \bar{R}_{P}\left(\phi_{P}, \theta_{P}, \psi_{P}\right)=R\left(Z^{\prime}, \phi_{P}\right) R\left(Y^{\prime}, \theta_{P}\right) R\left(Z^{\prime \prime}, \psi_{P}\right) .
$$

A primeira rotação se dá em torno do eixo $Z^{\prime}$ no valor do ângulo $\phi_{P}$ no sistema $X^{\prime}-Y^{\prime}-Z$ ' fixado a $\{P\}$. Em seguida, é dada uma rotação em torno do eixo $Y^{\prime}$ no valor do ângulo $\theta_{P}$ formando o novo sistema X"-Y"'-Z". Finalmente se dá uma última rotação do sistema em torno do novo eixo $Z^{\prime \prime}$ no valor de $\psi_{P}$ (Figura 2.4).

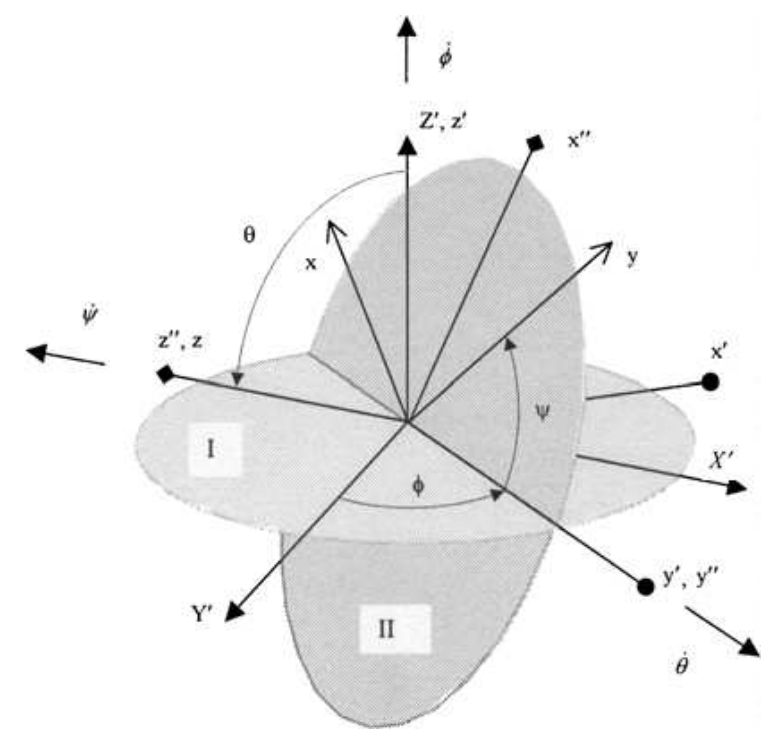

Figura 2.4: Rotações nos eixos Z-Y'-Z'. Fonte Harib e Srinivasan (2003a). 
Resolvendo (2.10) obtém-se:

$$
{ }^{o} \bar{R}_{P}=\left[\begin{array}{ccc}
c \phi_{P} c \theta_{P} c \psi_{P}-s \phi_{P} s \psi_{P} & -c \phi_{P} c \theta_{P} s \psi_{P}-s \phi_{P} c \psi_{P} & c \phi_{P} s \theta_{P} \\
s \phi_{P} c \theta_{P} c \psi_{P}+c \phi_{P} s \psi_{P} & -s \phi_{P} c \theta_{P} s \psi_{P}+c \phi_{P} s \psi_{P} & s \phi_{P} s \theta_{P} \\
-s \theta_{P} c \psi_{P} & s \theta_{P} s \psi_{P} & c \theta_{P}
\end{array}\right]
$$

A orientação da plataforma depende da orientação e posição final de cada perna do robô. Esta orientação é dada por (2.7) para cada perna e por sua vez tem

que satisfazer (2.11). Dada qualquer matriz de rotação $R_{3 \times 3}$, os ângulos de Euler produzidos pela rotação da matriz (2.11) podem ser calculados como segue:

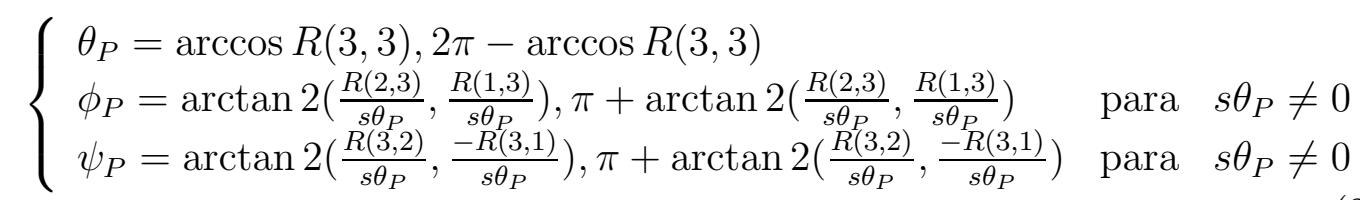

onde $R(x, y)$ é o elemento $x, y$ de $R$. No caso de $\theta_{P}=0$ ou $\theta_{P}=\pi$ a solução do sistema degenera. Neste caso somente a soma ou a diferença entre $\psi_{P}$ e $\phi_{P}$ podem ser calculadas. Igualando as matrizes (2.3) e (2.11) tem-se que:

$$
\begin{gathered}
c \theta_{P}=-c \theta_{2_{l} 3_{l}} \\
t \psi_{P}=t \theta_{1_{l}} \\
t \phi_{P}=t \theta_{4_{l}}
\end{gathered}
$$

\subsubsection{Solução do problema direto para a perna}

Ao contrário de como foi assumido na seção anterior, enquanto uma perna está no ar esta se comporta como um manipulador clássico de posicionamento (estrutura com um eixo vertical e dois eixos paralelos) com base no ponto $E_{l}$ e efetuador como sendo a garra. 
Uma vez que as matrizes de rotação são não-singulares, suas matrizes inversas existem e podem ser utilizadas para encontrar a matriz de transformação homogênea na direção da garra.

De acordo com a equação (2.2) temos que a partir do subproduto ${ }^{A_{l}} T_{E_{l}}$, a saber:

$$
{ }^{A_{l}} T_{E_{l}}={ }^{A_{l}} T_{B_{l}} \cdot{ }^{B_{l}} T_{C_{l}} \cdot{ }^{C_{l}} T_{D_{l}} \cdot{ }^{D_{l}} T_{E_{l}}
$$

pode ser encontrada a matriz de transformação.

$$
\begin{gathered}
{ }^{E_{l}} T_{A_{l}}={ }^{A_{l}} T_{E_{l}}^{-1} \\
{ }^{E_{l}} T_{A_{l}}=\left[\begin{array}{ccc}
{ }^{E_{l}} R_{A_{l}} & { }^{E_{l}} \overrightarrow{E_{l} A_{l}} \\
-- & + & -- \\
0 & \mid & 1
\end{array}\right]
\end{gathered}
$$

onde:

$$
{ }^{E_{l}} R_{A_{l}}=\left[\begin{array}{ccc}
c \theta_{4_{l}} c \theta_{2_{l} 3_{l}} c \theta_{1_{l}}+s \theta_{4_{l}} s \theta_{1_{l}} & s \theta_{4_{l}} c \theta_{2_{l} 3_{l}} c \theta_{1_{l}}-c \theta_{4_{l}} s \theta_{1_{l}} & c \theta_{1_{l}} s \theta_{2_{l} 3_{l}} \\
-c \theta_{4_{l}} c \theta_{2_{l} 3_{l}} s \theta_{1_{l}}+s \theta_{4_{l}} c \theta_{1_{l}} & -s \theta_{4_{l}} c \theta_{2_{l} 3_{l}} s \theta_{1_{l}}-c \theta_{4_{l}} c \theta_{1_{l}} & -s \theta_{1_{l}} s \theta_{2_{l} 3_{l}} \\
s \theta_{2_{l} 3_{l}} c \theta_{4_{l}} & s \theta_{2_{l} 3_{l}} s \theta_{4_{l}} & -c \theta_{2_{l} 3_{l}}
\end{array}\right]
$$

A posição da garra será dada no sistema $\left\{E_{l}\right\}$ pelo vetor:

$$
{ }^{E_{l}} \overrightarrow{E_{l} A_{l}}=\left[\begin{array}{c}
\left(c \theta_{2_{l} 3_{l}} \bar{L}_{4}+c \theta_{2_{l}} L_{3}+L_{2}\right) c \theta_{1_{l}} \\
\left(c \theta_{2_{l} 3_{l}} \bar{L}_{4}+c \theta_{2_{l}} L_{3}+L_{2}\right) s \theta_{1_{l}} \\
s \theta_{2_{l} 3_{l}} \bar{L}_{4}+s \theta_{2_{l}} L_{3}
\end{array}\right]
$$

onde $\bar{L}_{4}=L_{4}+L_{5}$.

$\mathrm{O}$ vetor ${ }^{E_{l}} \overrightarrow{E_{l} A_{l}}$ corresponde à solução do problema direto para a perna. Similarmente a como foi feito com o vetor ${ }^{O} \overrightarrow{B_{l} P}, E_{l} \overrightarrow{E_{l} A_{l}}$ faz um mapeamento direto entre o espaço das juntas e o espaço cartesiano de coordenadas X-Y-Z relativo ao sistema $\left\{E_{l}\right\}$. Semelhante ao problema da análise de posição da plataforma, temos 
que a garra do Kamambaré apresenta também 4 graus de liberdade. Estes graus de liberdade são as coordenadas de posição do ponto $A_{l}$ em relação sistema $\left\{E_{l}\right\}$, ${ }^{E_{l}} A_{x_{l}},{ }^{E_{l}} A_{y_{l}},{ }^{E_{l}} A_{z_{l}}$ e o ângulo de rotação da garra $\psi_{l}=\theta_{4_{l}}$.

O ângulo de rotação da garra $\psi_{l}=\theta_{4_{l}}$ pode ser encontrado igualando a matriz de rotação ${ }^{E_{l}} R_{A_{l}}$ com a matriz de rotação de Euler (convenção Z-Y'-Z") entre o sistema $\left\{E_{l}\right\}$ e o sistema da garra $\left\{A_{l}\right\}$, de forma similar a como foi feito com a orientação da plataforma.

\subsection{Análise de posição inversa}

No problema da análise inversa, a localização e orientação do corpo é dada e o problema consiste em encontrar os ângulos das juntas necessários para isso (Kolter et al., 2008). Esta análise é a mais usada no problema de controle, pois o objetivo é enviar os sinais necessários às juntas para levar o corpo ou a garra, a uma posição desejada.

Paralelamente à solução do problema inverso está o conceito de espaço de trabalho. O espaço de trabalho consiste no conjunto de pontos alcançáveis pelo corpo ou garra. Geralmente a relação entre o espaço cartesiano e o espaço das juntas não é unívoca, o que aumenta o grau de complexidade deste problema (Pieper, 1968).

\subsubsection{Solução do problema inverso para a plataforma}

O problema da análise inversa pode ser descrito da seguinte forma: dada uma determinada posição espacial do centro da plataforma e a orientação desta em relação ao sistema $\{O\}$, quais são os valores dos ângulos das juntas da perna que lhes correspondem (Spong e Vidyasagar, 1995; Staicua e Zhang, 2008)?

As entradas do nosso problema são agora a posição do centro da plataforma e sua orientação $\left[{ }^{O} P_{x},{ }^{O} P_{y},{ }^{O} P_{z}, \psi_{P}\right]$ e o ponto de agarramento da perna em questão 
$\left[{ }^{O} A_{x_{l}},{ }^{O} A_{y_{l}},{ }^{O} A_{z_{l}}\right]$. Note que os ângulos $\theta_{P}$ e $\phi_{P}$ não fazem parte dos GDL da plataforma pois eles resultam dos movimentos do ponto $P$ e do ângulo $\psi_{P}$ como será mostrado a continuação.

A equação de posição (2.8) pode ser expandida em três equações escalares:

$$
\begin{gathered}
x_{P A B_{l}}=\left(c \theta_{4_{l}} c \theta_{2_{l} 3_{l}} c \psi_{P}+s \theta_{4_{l}} s \psi_{P}\right) L_{1}+c \theta_{4_{l}}\left(c \theta_{2_{l} 3_{l}} L_{2}+c \theta_{3_{l}} L_{3}\right) \\
y_{P A B_{l}}=\left(s \theta_{4_{l}} c \theta_{2_{l} 3_{l}} c \psi_{P}-c \theta_{4_{l}} s \psi_{P}\right) L_{1}+s \theta_{4_{l}}\left(c \theta_{2_{l} 3_{l}} L_{2}+c \theta_{3_{l}} L_{3}\right) \\
z_{P A B_{l}}=s \theta_{2_{l} 3_{l}} c \psi_{P} L_{1}+s \theta_{2_{l} 3_{l}} L_{2}+s \theta_{3_{l}} L_{3}
\end{gathered}
$$

onde $x_{P A B_{l}}={ }^{O} P_{x}-{ }^{O} A_{x_{l}}-{ }^{O} B_{x_{l}}, y_{P A B_{l}}={ }^{O} P_{y}-O_{A_{y_{l}}}-{ }^{O} B_{y_{l}}$ e $z_{P A B_{l}}=$ ${ }^{O} P_{z}-{ }^{O} A_{z_{l}}-{ }^{O} B_{z_{l}}-L_{4}$

Reescrevendo (2.21) e (2.22):

$$
\begin{aligned}
& x_{P A B_{l}}=c \theta_{4_{l}}\left(c \theta_{2_{l} 3_{l}} c \psi_{P} L_{1}+c \theta_{2_{l} 3_{l}} L_{2}+c \theta_{3_{l}} L_{3}\right)+s \theta_{4_{l}} s \psi_{P} L_{1} \\
& y_{P A B_{l}}=s \theta_{4_{l}}\left(c \theta_{2_{l} 3_{l}} c \psi_{P} L_{1}+c \theta_{2_{l} 3_{l}} L_{2}+c \theta_{3_{l}} L_{3}\right)-c \theta_{4_{l}} s \psi_{P} L_{1}
\end{aligned}
$$

Para $c \theta_{4_{l}} \neq 0$ tem-se:

$$
\frac{\left(x_{P A B_{l}}-s \theta_{4_{l}} s \psi_{P}\right) L_{1}}{c \theta_{4_{l}}}=c \theta_{2_{l} 3_{l}} c \psi_{P} L_{1}+c \theta_{2_{l} 3_{l}} L_{2}+c \theta_{3_{l}} L_{3}
$$

Substituindo (2.26) em (2.25):

$$
c \theta_{4_{l}} y_{P A B_{l}}-s \theta_{4_{l}} x_{P A B_{l}}+s \psi_{P}^{2} L_{1}^{2}=0
$$

Elevando ao quadrado (2.27):

$$
c \theta_{4_{l}}^{2}\left(x_{P A B_{l}}^{2}+y_{P A B_{l}}^{2}\right)+2 c \theta_{4_{l}} y_{P A B_{l}} s \psi_{P} L_{1}+s \psi_{P}^{2} L_{1}^{2}-x_{P A B_{l}}^{2}=0 .
$$


Logo, aplicando a fórmula de Bhaskara em (2.28) tem-se:

$$
c \theta_{4_{l}}=\frac{y_{P A B_{l}} s \psi_{P} L_{1} \pm x_{P A B_{l}} \sqrt{x_{P A B_{l}}^{2}+y_{P A B_{l}}^{2}-s \psi_{P}^{2} L_{1}^{2}}}{x_{P A B_{l}}^{2}+y_{P A B_{l}}^{2}}
$$

sempre que:

$$
x_{P A B_{l}}^{2}+y_{P A B_{l}}^{2} \neq 0 .
$$

A equação (2.29) tem solução real para:

$$
x_{P A B_{l}}^{2}+y_{P A B_{l}}^{2} \geq s \psi_{P}^{2} L_{1}^{2}
$$

e o ângulo $\theta_{4_{l}}$ está definido no intervalo:

$$
\left|\frac{y_{P A B_{l}} s \psi_{P} L_{1} \pm x_{P A B_{l}} \sqrt{x_{P A B_{l}}^{2}+y_{P A B_{l}}^{2}-s \psi_{P}^{2} L_{1}^{2}}}{x_{P A B_{l}}^{2}+y_{P A B_{l}}^{2}}\right| \leq 1
$$

Trabalhando nesta última equação temos que a inequação é satisfeita para:

$$
x_{P A B_{l}}^{2}+y_{P A B_{l}}^{2}>0 .
$$

$\mathrm{e}$

$$
\left(y_{P A B_{l}}^{2}-s \psi_{P}^{2} L_{1}^{2}\right)^{2} \geq 0 .
$$

Estas duas últimas condições sempre são satisfeitas.

Para o cálculo de $\theta_{2_{l}}$, são elevadas ao quadrado as equações $(2.21)$, (2.22) e, em seguida somadas:

$$
\left(c \theta_{2_{l} 3_{l}} c \psi_{P} L_{1}+c \theta_{2_{l} 3_{l}} L_{2}+c \theta_{3_{l}} L_{3}\right)^{2}=x_{P A B_{l}}^{2}+y_{P A B_{l}}^{2}+s \psi_{P}^{2} L_{1}^{2} .
$$


Se agora elevamos ao quadrado (2.23) e a somamos a (2.35) o resultado será:

$$
\bar{L}_{2}^{2}+2 \bar{L}_{2} L_{3}\left(c \theta_{2_{l} 3_{l}} c \theta_{3}+s \theta_{2_{l} 3_{l}} s \theta_{3}\right)+L_{3}^{2}=x_{P A B_{l}}^{2}+y_{P A B_{l}}^{2}+z_{P A B_{l}}^{2}+s \psi_{P}^{2} L_{1}^{2} .
$$

Como $c \theta_{2_{l} 3_{l}} c \theta_{3}+s \theta_{2_{l} 3_{l}} s \theta_{3}=c \theta_{2_{l}}$, finalmente:

$$
c \theta_{2_{l}}=\frac{x_{P A B_{l}}^{2}+y_{P A B_{l}}^{2}+z_{P A B_{l}}^{2}-L_{3}^{2}-\bar{L}_{2}^{2}-s \psi_{P}^{2} L_{1}^{2}}{2 L_{3} \bar{L}_{2}}
$$

onde $\bar{L}_{2}=c \psi_{P} L_{1}+L_{2}$.

Como $\left|c \theta_{2_{l}}\right| \leq 1$, a equação (2.37) está sujeita às seguintes restrições:

$$
x_{P A B_{l}}^{2}+y_{P A B_{l}}^{2}+z_{P A B_{l}}^{2} \leq\left(L_{3}+\bar{L}_{2}\right)^{2}+2 L_{1} c \psi_{P}\left(L_{3}+\bar{L}_{2}\right)+L_{1}^{2}
$$

e

$$
x_{P A B_{l}}^{2}+y_{P A B_{l}}^{2}+z_{P A B_{l}}^{2} \geq\left(L_{3}-\bar{L}_{2}\right)^{2}-2 L_{1} c \psi_{P}\left(L_{3}-\bar{L}_{2}\right)+L_{1}^{2} .
$$

Finalmente utilizando a equação relativa a (2.23) e os valores já calculados de $\theta_{2_{l}}$ e $\theta_{4_{l}}$ é colocado em evidência $s \theta_{3_{l}}$ :

$$
s \theta_{2_{l}} c \theta_{3_{l}} \bar{L}_{2}+s \theta_{3_{l}} \bar{L}_{3}=z_{P A B_{l}}
$$

onde $\bar{L}_{3}=c \theta_{2 l} \bar{L}_{2}+L_{3}$.

Por fim, elevando ao quadrado a expressão (2.40) e agrupando de forma conveniente:

$$
s \theta_{3_{l}}^{2}\left(\bar{L}_{3}^{2}+\bar{L}_{2}^{2} s \theta_{2_{l}}^{2}\right)-2 z_{P A B_{l}} \bar{L}_{3} s \theta_{3_{l}}+z_{P A B_{l}}^{2}-\bar{L}_{2}^{2} s \theta_{2_{l}}^{2}=0
$$

Aplicando a fórmula de Bhaskara a (2.41): 


$$
s \theta_{3_{l}}=\frac{\left(c \theta_{2_{l}} \bar{L}_{2}+L_{3}\right) z_{P A B_{l}} \pm s \theta_{2_{l}} \bar{L}_{2} \sqrt{L_{3}^{2}+2 c \theta_{2_{l}} L_{3} \bar{L}_{2}+\bar{L}_{2}^{2}-z_{P A B_{l}}^{2}}}{L_{3}^{2}+2 c \theta_{2_{l}} L_{3} \bar{L}_{2}+\bar{L}_{2}^{2}}
$$

onde:

$$
\begin{gathered}
L_{3}^{2}+2 c \theta_{2_{l}} L_{3} \bar{L}_{2}+\bar{L}_{2}^{2}-z_{P A B_{l}}^{2} \geq 0, \\
L_{3}^{2}+2 c \theta_{2_{l}} L_{3} \bar{L}_{2}+\bar{L}_{2}^{2} \neq 0,
\end{gathered}
$$

e

$$
\left|\frac{\left(c \theta_{2_{l}} \bar{L}_{2}+L_{3}\right) z_{P A B_{l}} \pm s \theta_{2_{l}} \bar{L}_{2} \sqrt{L_{3}^{2}+2 c \theta_{2_{l}} L_{3} \bar{L}_{2}+\bar{L}_{2}^{2}-z_{P A B_{l}}^{2}}}{L_{3}^{2}+2 c \theta_{2_{l}} L_{3} \bar{L}_{2}+\bar{L}_{2}^{2}}\right| \leq 1 .
$$

Faremos agora uma análise das inequações (2.43), (2.44) e (2.45).

No caso de (2.43) isolamos $c \theta_{2_{l}}$ :

$$
c \theta_{2_{l}} \geq \frac{z_{P A B_{l}}^{2}-L_{3}^{2}-\bar{L}_{2}^{2}}{2 \bar{L}_{2} L_{3}} .
$$

Igualando (2.37) e (2.46) fica que (2.43) é satisfeita sempre que seja verificada $(2.31)$.

Já no caso de (2.44) a análise é feita diretamente. Logo:

$$
c \theta_{2_{l}} \neq-\frac{L_{3}^{2}+\bar{L}_{2}^{2}}{2 L_{3} \bar{L}_{2}} .
$$

De (2.43) e (2.44) tem-se que $z_{P A B_{l}} \neq 0$.

Finalmente resolvendo (2.45) obteve-se:

$$
\left(z_{P A B_{l}}-c \theta_{2_{l}} \bar{L}_{2}-L_{3}\right)^{2} \leq 0 .
$$


Utilizando agora o resultado de (2.48) em (2.43):

$$
\left(c \theta_{2_{l}} \bar{L}_{2}+L_{3}\right)^{2} \leq L_{3}^{2}+2 c \theta_{2_{l}} L_{3} \bar{L}_{2}+\bar{L}_{2}^{2}
$$

Resolvendo (2.49) tem-se que $s \theta_{2_{l}}^{2} L_{2}^{2} \geq 0$, condição que sempre está satisfeita.

Nesta seção ficou demonstrada a afirmação feita no inicio da seção em relação aos graus de liberdade do sistema. As equações (2.29), (2.37) e (2.42) demonstram a afirmação de que a partir da posição do centro da plataforma e do ângulo de orientação $\psi_{P}$, podem ser calculados os valores das demais juntas. Note que o valor do ângulo da plataforma $\psi_{P}=\theta_{1_{l}}$.

Uma vez resolvido o problema inverso, pode ser calculado o espaço de trabalho da plataforma, ou seja, aqueles pontos alcançáveis pelo mecanismo. Para isto serão usadas as restrições obtidas das equações. Basicamente as restrições que definem o espaço de trabalho da plataforma são (2.38) e (2.39), já que as demais estão contidas dentro dessas duas. A solução geométrica que satisfaz (2.38) e (2.39) é o espaço existente entre as duas semi-esferas concêntricas dadas por (2.38) e (2.39). O espaço de trabalho do ponto ${ }^{O} P$ associado à perna $l$ será representado pelo conjunto $\mathcal{W}_{P_{l}}$.

Quando mais de uma perna estiver sustentando o peso do robô, o espaço de trabalho final será definido pela intersecção dos espaços de trabalho das pernas fixas à superfície de apoio.

A Figura 2.5 mostra a representação dos conjuntos formados pelos espaços de trabalhos do ponto ${ }^{O} P$ relativos às pernas 1 e 3 . As coordenadas dos pontos de agarramento escolhidos foram ${ }^{O} A_{1}=[0,0,0]$ e ${ }^{O} A_{3}=[0.9243,0.4243,0]$, e os comprimentos dos ligamentos são os mostrados na Tabela 2.2. Para ambos conjuntos foram destacados os valores mínimos e máximos $\left(\mathcal{W}_{P l_{\min }}\right.$ e $\left.\mathcal{W}_{P l_{\max }}\right)$. 


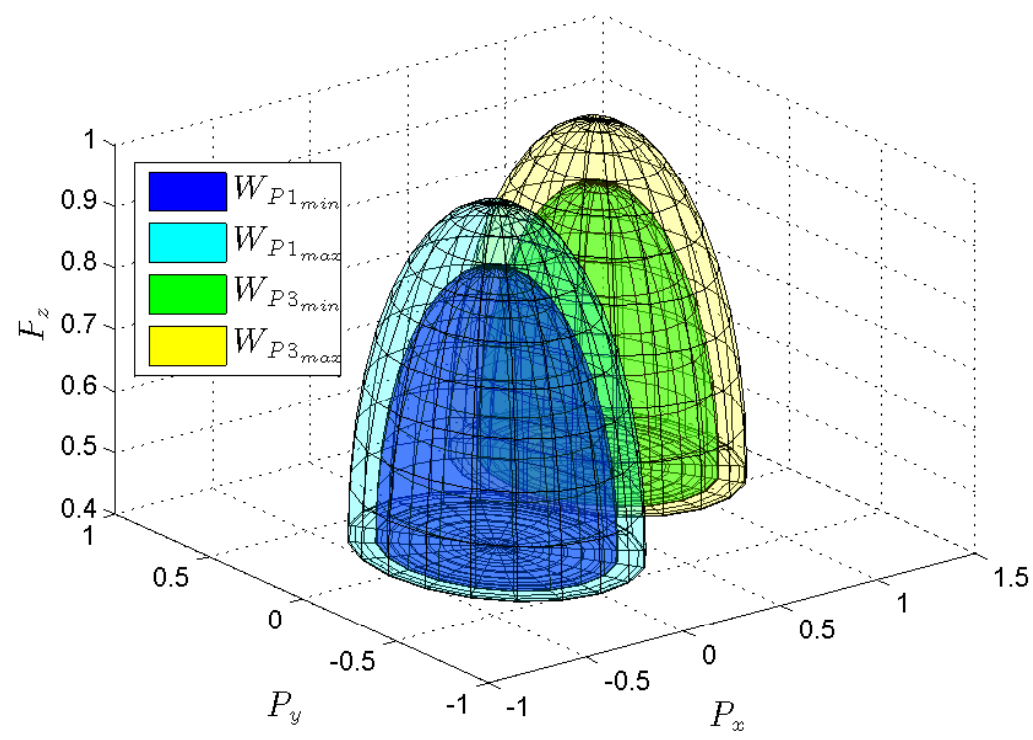

Figura 2.5: Espaço de trabalho do ponto ${ }^{O} P$ da plataforma para $0.24 \leq \psi_{P} \leq 1.32$ quando as pernas 1 e 3 estão fixas à superfície de apoio. $\left(\mathcal{W}_{P}=\mathcal{W}_{P_{1}} \cap \mathcal{W}_{P_{3}}\right)$.

\subsubsection{Solução do problema inverso para a perna}

De forma similar ao exposto na seção anterior será resolvido agora o problema inverso para quando a perna está no ar. O ponto de início de nosso trabalho será a equação (2.20).

Se o ponto $E_{l}$ é conhecido e o ponto $A_{l}$ de destino também, a equação (2.20) pode ser reescrita no sistema $\left\{E_{l}\right\}$ em forma escalar como:

$$
\begin{aligned}
& x_{A E_{l}}=\left(c \theta_{2_{l} 3_{l}} \bar{L}_{4}+c \theta_{2_{l}} L_{3}+L_{2}\right) c \theta_{1_{l}} \\
& y_{A E_{l}}=\left(c \theta_{2_{l} 3_{l}} \bar{L}_{4}+c \theta_{2_{l}} L_{3}+L_{2}\right) s \theta_{1_{l}}
\end{aligned}
$$

e

$$
z_{A E_{l}}=s \theta_{2_{l} 3_{l}} \bar{L}_{4}+s \theta_{2_{l}} L_{3}
$$


Tabela 2.2: Comprimento dos ligamentos das pernas

\begin{tabular}{cc} 
Ligamento & Dimensões $(\mathrm{m})$ \\
\hline$L_{5}$ & 0.2 \\
$L_{4}$ & 0.2 \\
$L_{3}$ & 0.3 \\
$L_{2}$ & 0.1 \\
$L_{1}$ & 0.3 \\
\hline
\end{tabular}

onde $x_{A E_{l}}={ }^{E_{l}} A_{x_{l}}-{ }^{E_{l}} E_{x_{l}}, y_{A E_{l}}={ }^{E_{l}} A_{y_{l}}-{ }^{E_{l}} E_{y_{l}}$ e $z_{A E_{l}}={ }^{E_{l}} A_{z_{l}}-{ }^{E_{l}} E_{z_{l}}$.

Logo, utilizando as equações (2.50) e (2.51) tem-se:

$$
t \theta_{1_{l}}=\frac{y_{A E_{l}}}{x_{A E_{l}}}
$$

desde que $c \theta_{1_{l}} \neq 0$ e $x_{A E_{l}} \neq 0$.

Depois de calculado o ângulo $\theta_{1_{l}}$, o próximo passo é calcular o valor de $\theta_{3_{l}}$. O procedimento é similar ao utilizado para o cálculo do ângulo $\theta_{2_{l}}$ no caso do problema inverso da plataforma, ou seja, as equações $(2.50)$, (2.51) e (2.52) são elevadas ao quadrado e posteriormente somadas. Finalmente depois de realizar estas operações o resultado é:

$$
c \theta_{3_{l}}=\frac{x_{A E_{l}}^{2}+y_{A E_{l}}^{2} \pm 2 L_{2} \sqrt{x_{A E_{l}}^{2}+y_{A E_{l}}^{2}}+z_{A E_{l}}^{2}+L_{2}^{2}-\bar{L}_{4}^{2}-L_{3}^{2}}{2 \bar{L}_{4} L_{3}} .
$$

Como $\left|c \theta_{3_{l}}\right| \leq 1$, a equação (2.54) tem solução real para:

$$
\left(\bar{L}_{4}+L_{3}\right)^{2} \leq\left(\sqrt{x_{A E_{l}}^{2}+y_{A E_{l}}^{2}}-L_{2}\right)^{2}+z_{A E_{l}}^{2} \leq\left(\bar{L}_{4}-L_{3}\right)^{2}
$$

Por último, para o cálculo da junta $\theta_{2_{l}}$ é utilizada a equação (2.52). 


$$
s \theta_{2_{l}}=\frac{z_{A E_{l}}\left(c \theta_{3_{l}} \bar{L}_{4}+L_{3}\right) \pm s \theta_{3_{l}} \bar{L}_{4} \sqrt{\bar{L}_{4}^{2}+2 c \theta_{3_{l}} \bar{L}_{4} L_{3}+L_{3}^{2}-z_{A E_{l}}^{2}}}{\bar{L}_{4}^{2}+2 c \theta_{3_{l}} L_{3} \bar{L}_{4}+L_{3}^{2}}
$$

onde para que exista solução real:

$$
\begin{gathered}
\bar{L}_{4}^{2}+2 c \theta_{3_{l}} \bar{L}_{4} L_{3}+L_{3}^{2} \neq 0, \\
\bar{L}_{4}^{2}+2 c \theta_{3_{l}} \bar{L}_{4} L_{3}+L_{3}^{2}-z_{A E_{l}}^{2} \geq 0
\end{gathered}
$$

e

$$
\left|\frac{z_{A E_{l}}\left(c \theta_{3_{l}} \bar{L}_{4}+L_{3}\right) \pm s \theta_{3_{l}} \bar{L}_{4} \sqrt{\bar{L}_{4}^{2}+2 c \theta_{3_{l}} \bar{L}_{4} L_{3}+L_{3}^{2}-z_{A E_{l}}^{2}}}{\bar{L}_{4}^{2}+2 c \theta_{3_{l}} L_{3} \bar{L}_{4}+L_{3}^{2}}\right| \leq 1 .
$$

Resolvendo (2.57) tem-se:

$$
c \theta_{3_{l}} \neq-\frac{\bar{L}_{4}^{2}+L_{3}^{2}}{2 \bar{L}_{4} L_{3}} .
$$

De (2.57) e (2.58) deduce-se que $z_{A E_{l}} \neq 0$. A configuração $z_{A E_{l}}=0$ equivale a ter a perna completamente esticada na horizontal.

Resolvendo $\operatorname{logo}(2.58)$ em função de $c \theta_{3_{l}}$ e substituindo este valor em (2.54) temos:

$$
x_{A E_{l}}^{2}+y_{A E_{l}}^{2} \geq L_{2}^{2} .
$$

Finalmente da equação (2.59) obteve-se que $\left(z_{A E_{l}}-c \theta_{3_{l}} \bar{L}_{4}-L_{3}\right)^{2} \leq 0$. Colocando este valor em (2.58) tem-se que $s \theta_{3_{l}}^{2} \bar{L}_{4}^{2} \geq 0$, condição sempre satisfeita.

O ângulo $\theta_{4_{l}}$ não influencia na posição do ponto de agarramento, mas sim na orientação da garra (seu quarto grau de liberdade), assim $\psi_{l}=\theta_{4_{l}}$. 
O espaço de trabalho do ponto ${ }^{E_{l}} A_{l}$ enquanto a perna está no ar $\mathcal{W}_{A_{l}}$ pode ser representado pelo conjunto de pontos que formam a solução da equação (2.54), sujeito às restrições impostas pelas inequações (2.55) e (2.57). Geometricamente o conjunto $\mathcal{W}_{A_{l}}$ é mostrado na Figura 2.6. Nesta figura os comprimentos dos ligamentos utilizados são os mostrados na Tabela 2.2 e as coordenadas da base da perna são ${ }^{O} E_{l}=[0.2,0.15,0.23]$.

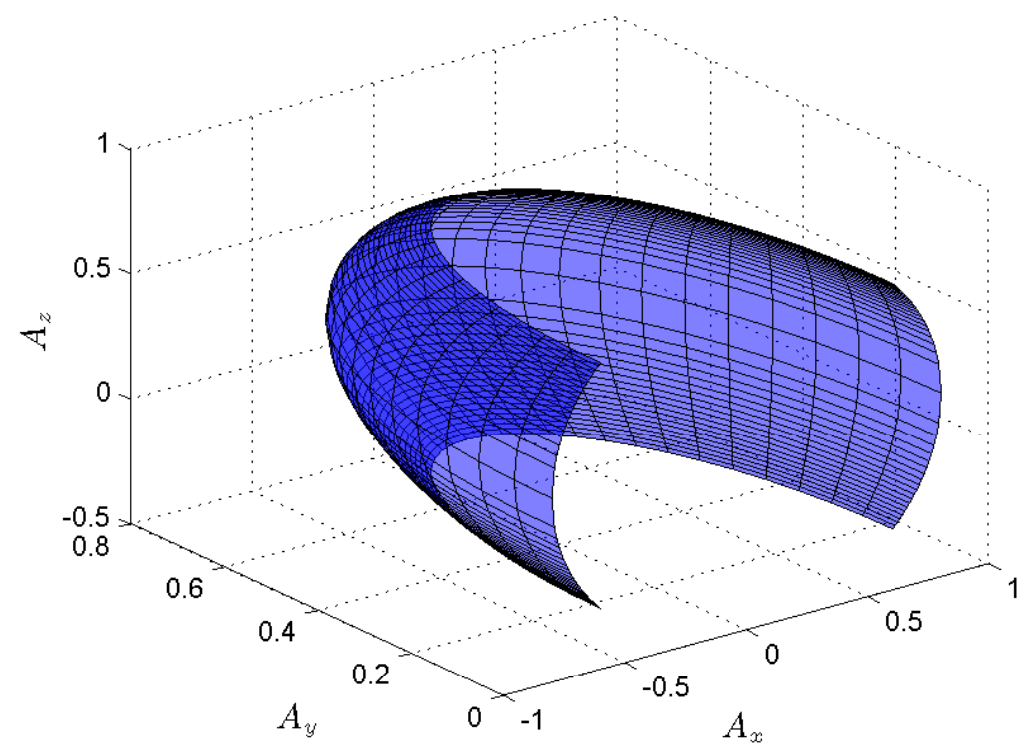

Figura 2.6: Espaço de trabalho da garra $l$ no ar. 


\section{Capítulo 3}

\section{Cinemática}

\subsection{Introdução}

A cinemática é o ramo da mecânica que estuda o movimento dos corpos sem analisar as forças que o causam (Craig, 1989). No caso de um robô a cinemática ainda descreve a relação entre o movimento das juntas em cada articulação e o movimento resultante das partes rígidas de seu corpo (Figura 3.1).

No capítulo anterior foi feita uma análise dos problemas direto e inverso. Esta análise é importante ao se deslocar a plataforma ou as pernas do robô até uma posição e orientação desejada no espaço, mas em nosso caso não é suficiente. Matematicamente as soluções direta e inversa definem uma função ou mapeamento entre o espaço das juntas e o espaço cartesiano e vice-versa. Já a relação entre as velocidades nestes espaços é dada pelo Jacobiano desta função. A matriz jacobiana é um dos elementos mais importantes a analisar no controle de movimento em sistemas mecânicos.

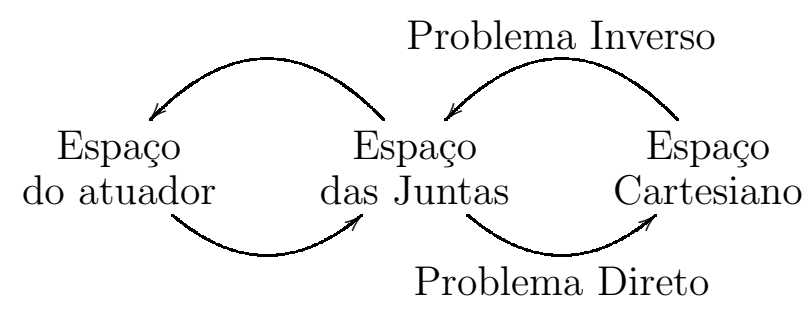

Figura 3.1: Mapeamento entre os diferentes espaços de estados. 
Um ponto importante na cinemática corresponde ao estudo das singularidades apresentadas pelo robô. O problema das singularidades tem sido amplamente estudado para manipuladores de cadeia aberta como em Hunt (1986); Craig (1989); Kumar (1990); Spong e Vidyasagar (1995); Zlatanov et al. (1994a); Tsai (1999) e outros. No entanto, estudos de singularidades em cadeias fechadas são escassos. Zlatanov et al. (1994b) propõem uma estrutura geral com seis tipos de singularidades diferentes, analisadas a partir de um subespaço linear chamado espaço de movimento dentro do espaço da velocidade, porém o método mais utilizado na literatura é o apresentado por Goselin e Angeles (1990). Este método é baseado nas derivadas das entradas e saídas do sistema e da origem a três tipos diferentes de singularidades para robôs paralelos. Aplicações desta metodologia podem ser encontradas em Tsai (1999); Harib e Srinivasan (2003b); Tartari e Cabral (2006); Merlet (2006); Potts e Da Cruz (2010). Precisamente este será o método utilizado na análise das singularidades neste capítulo. Similarmente ao que foi feito no capítulo anterior, as análises relativas à plataforma e à perna serão tratadas de forma separada.

\subsection{Análise cinemática de velocidade da plata- forma}

Enquanto a velocidade linear descreve a taxa de variação da posição de um ponto da plataforma no espaço, o vetor velocidade angular $\vec{\omega}$ caracteriza a variação da orientação da plataforma no tempo.

Como já foi visto em (2.11), a orientação do sistema de coordenadas preso à plataforma com relação ao sistema $\{O\}$ pode ser descrita pela matriz de rotação ${ }^{O} R_{P}$. Como esta matriz é ortogonal, a transformação inversa de ${ }^{O} R_{P}$ é idêntica à sua transposta e, por tanto:

$$
{ }^{O} R_{P} \cdot{ }^{O} R_{P}^{T}=I
$$


Derivando (3.1) com relação ao tempo temos:

$$
\begin{gathered}
{ }^{O} \dot{R}_{P}{ }^{O}{ }^{O} R_{P}^{T}+{ }^{O} R_{P} \cdot{ }^{O} \dot{R}_{P}^{T}=0 . \\
\text { Substituindo }{ }^{O} R_{P}^{T}={ }^{O} R_{P}^{-1} \text { e }{ }^{O} R_{P}=\left({ }^{O} R_{P}^{-1}\right)^{T} \text { em }(3.2): \\
\left({ }^{O} \dot{R}_{P} \cdot{ }^{O} R_{P}^{-1}\right)+\left({ }^{O} R_{P} \cdot{ }^{O} \dot{R}_{P}^{-1}\right)^{T}=0
\end{gathered}
$$

o que significa que ${ }^{O} \dot{R}_{P} \cdot{ }^{O} R_{P}^{-1}$ é uma matriz anti-simétrica de dimensão $3 \times 3$. Sem perder generalidade, esta pode se definida como:

$$
\Omega \equiv{ }^{O} \dot{R}_{P} \cdot{ }^{O} R_{P}^{-1}=\left[\begin{array}{ccc}
0 & -\omega_{p_{z}} & \omega_{p_{y}} \\
\omega_{p_{z}} & 0 & -\omega_{p_{x}} \\
-\omega_{p_{y}} & \omega_{p_{x}} & 0
\end{array}\right]
$$

onde, ${ }^{O} \omega_{p_{x}},{ }^{O} \omega_{p_{y}}$ e ${ }^{O} \omega_{p_{z}}$ são três parâmetros independentes que especificam a velocidade angular da plataforma com relação ao sistema $\{O\}$. Em seguida, em função dos ângulos de Euler $\theta_{P}, \psi_{P}$ e $\phi_{P}$, pode ser formulada a seguinte equação:

$$
\left[\begin{array}{c}
O \omega_{p_{x}} \\
O \omega_{p_{y}} \\
{ }^{O} \omega_{p_{z}}
\end{array}\right]=\left[\begin{array}{ccc}
0 & -s \phi_{P} & s \theta_{P} c \phi_{P} \\
0 & c \phi_{P} & s \theta_{P} s \phi_{P} \\
1 & 0 & c \theta_{P}
\end{array}\right]\left[\begin{array}{c}
\dot{\phi_{P}} \\
\dot{\theta_{P}} \\
\dot{\psi_{P}}
\end{array}\right]
$$

Para calcular a velocidade linear do ponto $E_{l}$ em relação ao sistema $\{O\}$ temos de recorrer à equação (2.8). Derivando esta equação em relação ao tempo obtemos:

$$
{ }^{O} \vec{v}_{E_{l}}={ }^{O} \vec{v}_{P}+{ }^{O} \vec{\omega}_{p} \times\left({ }^{O} R_{P} \cdot{ }^{O} P \vec{E}_{l}\right) .
$$

A parte esquerda de (3.6) é obtida derivando (2.9) com relação ao tempo:

$$
{ }^{O} \vec{v}_{E_{l}}=J_{q_{l}}\left[\begin{array}{c}
\dot{\theta}_{4_{l}} \\
\dot{\theta}_{3 l} \\
\dot{\theta}_{2_{l}}
\end{array}\right]
$$


onde:

$$
J_{q_{l}}=\left[\begin{array}{ccc}
-s \theta_{4_{l}}\left(c \theta_{3_{l} 2_{l}} L_{2}+c \theta_{3_{l}} L_{3}\right) & -c \theta_{4_{l}}\left(s \theta_{3_{l} 2_{l}} L_{2}+s \theta_{3_{l}} L_{3}\right) & -c \theta_{4_{l}} L_{2} s \theta_{3_{l} 2_{l}} \\
c \theta_{4_{l}}\left(c \theta_{3_{l} 2_{l}} L_{2}+c \theta_{3_{l}} L_{3}\right) & -s \theta_{4_{l}}\left(s \theta_{3_{l} 2_{l}} L_{2}+s \theta_{3_{l}} L_{3}\right) & -s \theta_{4_{l}} L_{2} s \theta_{3_{l} 2_{l}} \\
0 & c \theta_{3_{l} 2_{l}} L_{2}+c \theta_{3_{l}} L_{3} & c \theta_{3_{l} 2_{l}} L_{2}
\end{array}\right] .
$$

Do lado direito de (3.6) vemos que o produto vetorial ${ }^{O} \vec{\omega}_{p} \times\left({ }^{O} R_{P} \cdot{ }^{O} P \vec{E}_{l}\right)$ pode ser escrito em forma matricial como o produto de duas matrizes:

$$
O_{\overrightarrow{\omega_{p}}} \times\left({ }^{O} R_{P} \cdot{ }^{O} P \overrightarrow{E_{l}}\right)=\Omega_{F_{l}}\left[\begin{array}{c}
O_{\omega_{p_{x}}} \\
O_{\omega_{p_{y}}} \\
O_{\omega_{p_{z}}}
\end{array}\right]
$$

onde:

$$
\Omega_{F_{l}}=\left[\begin{array}{ccc}
0 & \Upsilon_{z_{l}} & -\Upsilon_{y_{l}} \\
-\Upsilon_{z_{l}} & 0 & \Upsilon_{x_{l}} \\
\Upsilon_{y_{l}} & -\Upsilon_{x_{l}} & 0
\end{array}\right]
$$

e $\vec{\Upsilon}_{l}={ }^{O} R_{P} \cdot{ }^{O} P \vec{E}_{l}$.

Substituindo (3.5) e (3.9) em (3.6):

$$
{ }_{{ }^{\prime}} \vec{v}_{E_{l}}=J_{x_{1}} J_{x_{2}}\left[\begin{array}{c}
O v_{P_{x}} \\
O_{P_{y}} \\
{ }_{v_{P_{z}}} \\
\dot{\phi} \\
\dot{\theta} \\
\dot{\psi}
\end{array}\right]
$$

onde:

$$
J_{x_{1}}=\left[\begin{array}{ll}
I_{3 \times 3} & \Omega_{F_{l}}
\end{array}\right]
$$

e

$$
J_{x_{2}}=\left[\begin{array}{c}
I_{3 \times 3} \\
0_{3 \times 3}
\end{array} \quad\left[\begin{array}{ccc}
0 & -s \phi_{P} & s \theta_{P} c \phi_{P} \\
0 & c \phi_{P} & s \theta_{P} s \phi_{P} \\
1 & 0 & c \theta_{P}
\end{array}\right]\right]
$$


Finalmente igualando as equações (3.11) e (3.7) para as quatro pernas temos:

$$
J_{q} \overrightarrow{\dot{q}}=J_{x} \overrightarrow{\dot{x}}
$$

onde:

$$
\begin{aligned}
& J_{x}=J_{x_{1}} J_{x_{2}}, \\
& J_{q}=\left[\begin{array}{cccc}
J_{q_{1}} & 0_{3 \times 3} & \cdots & 0_{3 \times 3} \\
0_{3 \times 3} & J_{q_{2}} & \ddots & \vdots \\
\vdots & \ddots & J_{q_{3}} & 0_{3 \times 3} \\
0_{3 \times 3} & \cdots & 0_{3 \times 3} & J_{q_{4}}
\end{array}\right], \\
& J_{x_{1}}=\left[\begin{array}{cc}
I_{3 \times 3} & \Omega_{F_{1}} \\
\vdots & \vdots \\
I_{3 \times 3} & \Omega_{F_{4}}
\end{array}\right] \text {, } \\
& \vec{q}=\left[\theta_{4_{1}}, \theta_{3_{1}}, \theta_{2_{1}} \ldots \theta_{4_{4}}, \theta_{3_{4}}, \theta_{2_{4}}\right]^{T}
\end{aligned}
$$

$\mathrm{e}$

$$
\vec{x}=\left[{ }^{O} P_{x},{ }^{O} P_{y},{ }^{O} P_{z}, \phi_{P}, \theta_{P}, \psi_{P}\right]^{T} .
$$

O vetor de entrada $\vec{q}$ está associado ao conjunto das juntas ativas, ou seja, aquelas que, acionadas pela lei de controle, produzirão o movimento do robô. Este conjunto pode variar de acordo com a andadura, com a quantidade de pernas fixas à superfície e com o algoritmo de otimização de energia utilizado. Num caso particular da andadura, todas as pernas podem estar agarradas à superfície e todas as juntas podem ser ditas ativas.

O vetor de saída $\vec{x}$ corresponde à posição e à orientação da plataforma. No caso em que as dimensões do vetor de entrada sejam maiores que as do vetor de saída, têm-se redundâncias (Goselin e Angeles, 1990). Os parâmetros ${ }^{O} P_{x},{ }^{O} P_{y},{ }^{O} P_{z}, \phi_{P}, \theta_{P}$ e $\psi_{P}$ do vetor $\vec{x}$ não são todos arbitrários, pois eles têm que respeitar as restrições cinemáticas impostas pelas equações (2.29) a (2.37) e (2.14) a (2.15). 


\subsubsection{Singularidade inversa da plataforma}

O estudo de singularidades em robôs paralelos é muito mais difícil que o estudo para manipuladores de cadeia aberta, devido principalmente à grande quantidade de caminhos fechados que podem existir entre as diferentes pernas do robô. Um fato a destacar na análise das singularidades de um sistema paralelo é que estas podem se apresentar tanto na fronteira como no interior do espaço de trabalho.

Alguns autores como Goselin e Angeles (1990); Tsai (1999) costumam dividir a matriz jacobiana em duas matrizes, a primeira associada com a cinemática direta e a outra associada com a cinemática inversa. Dependendo de qual matriz é singular, o mecanismo pode apresentar singularidade na sua cinemática direta, na sua cinemática inversa ou em ambas. Este último enfoque foi o seguido nesta seção.

As matrizes $J_{q}$ e $J_{x}$ podem ser reformuladas como $J_{q}=\frac{\partial \vec{f}}{\partial \vec{q}}$ e $J_{x}=\frac{\partial \vec{f}}{\partial \vec{x}}$ onde $\vec{f}$ é uma função implícita n-dimensional de $\vec{q}$ e $\vec{x}$ que representa a relação entre o conjunto de variáveis de entrada e o conjunto de variáveis de saída do sistema segundo:

$$
\vec{f}(\vec{x}, \vec{q})=\overrightarrow{0}
$$

Analisando desta forma, as matrizes $J_{q}$ e $J_{x}$ são as matrizes jacobianas de $\vec{f}$. As singularidades na configuração acontecem quando uma destas matrizes ou ambas são singulares. Logo, em robôs paralelos, existem três tipos de singularidades, cada uma delas com uma determinada interpretação física.

O primeiro tipo de singularidade acontece quando $\operatorname{det}\left(J_{q}\right)=0$. Mecanicamente esta singularidade significa que a cadeia cinemática alcançou a fronteira do espaço de trabalho ou uma outra fronteira limitada pelas diferentes sub-regiões internas do espaço de trabalho onde as diferentes soluções da cinemática inversa convergem.

De (3.16) tem-se que: 


$$
\operatorname{det}\left(J_{q}\right)=\operatorname{det}\left(J_{q_{1}}\right) \operatorname{det}\left(J_{q_{2}}\right) \operatorname{det}\left(J_{q_{3}}\right) \operatorname{det}\left(J_{q_{4}}\right)
$$

onde

$$
\operatorname{det}\left(J_{q_{l}}\right)=-L_{2} L_{3} s \theta_{2_{l}}\left(c \theta_{2_{l} 3_{l}} L_{2}+c \theta_{3_{l}} L_{3}\right)
$$

para $l=1,2, \ldots, 4$.

As singularidades ocorrem se $\theta_{2_{l}}=0, \pm \pi, \ldots, \pm n \pi, \forall n \in \mathbb{N}$ ou

$$
c \theta_{3_{l}}= \pm \frac{\left|s \theta_{2_{l}}\right|}{\sqrt{\frac{L_{3}^{2}}{L_{2}^{2}}+2 \frac{L_{3}}{L_{2}} c \theta_{2_{l}}+1}}
$$

para:

$$
c \theta_{2_{l}}>-\frac{\frac{L_{3}^{2}}{L_{2}^{2}}+1}{2 \frac{L_{3}}{L_{2}}} .
$$

De acordo com (3.23), para um dado valor de $\theta_{2_{l}}$ existirão duas soluções de $\theta_{3 l}$.

No primeiro caso, $\theta_{2_{l}}=0, \pm \pi, \ldots, \pm n \pi$ significa que a barra $L_{2}$ se encontra completamente alinhada com $L_{3}$. Já no segundo caso a singularidade acontece quando os pontos $E_{l}, C_{l}$ e $B_{l}$ encontram-se alinhados verticalmente. Estas duas situações são apresentadas nas Figuras 3.2 e 3.3, onde as pernas 1 e 3 estão fixas à superfície de apoio.

Nestas configurações o robô perde um ou mais graus de liberdade, podendo resistir a grandes forças ou momentos em algumas direções sem nenhuma força ou torque aplicado nos atuadores. Na Figura 3.3, nos dois casos apresentados, os eixos das três juntas de cada perna são coplanares. Sempre que existam três eixos paralelos ou coplanares existirá uma singularidade na configuração do robô (Murray et al., 1994). 


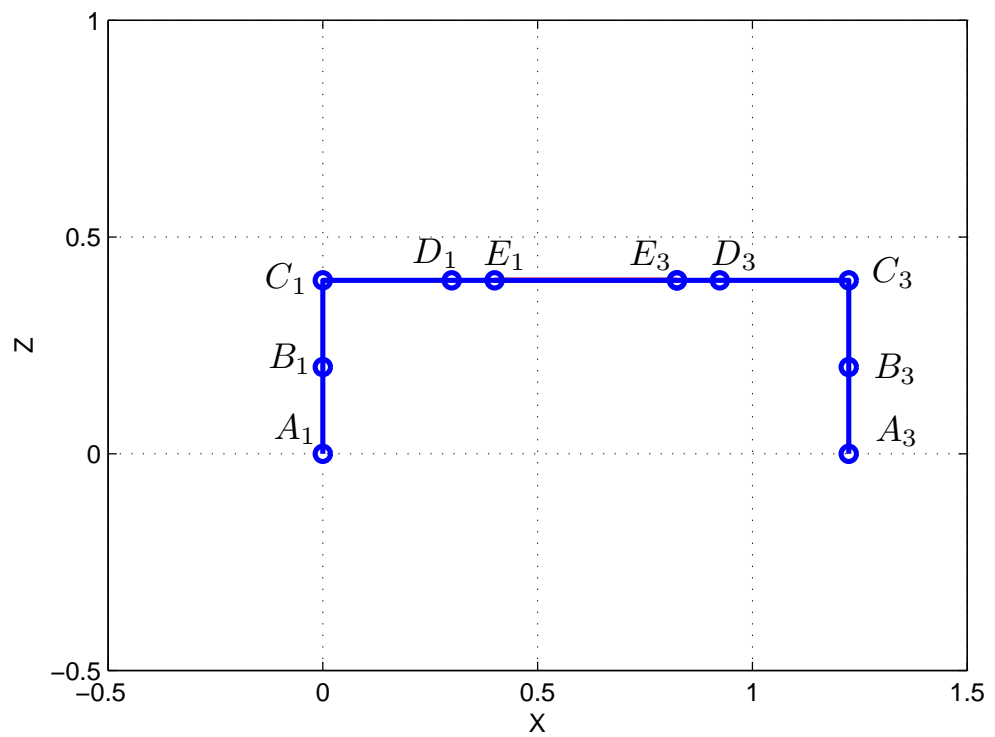

Figura 3.2: Primeiro caso de singularidade.

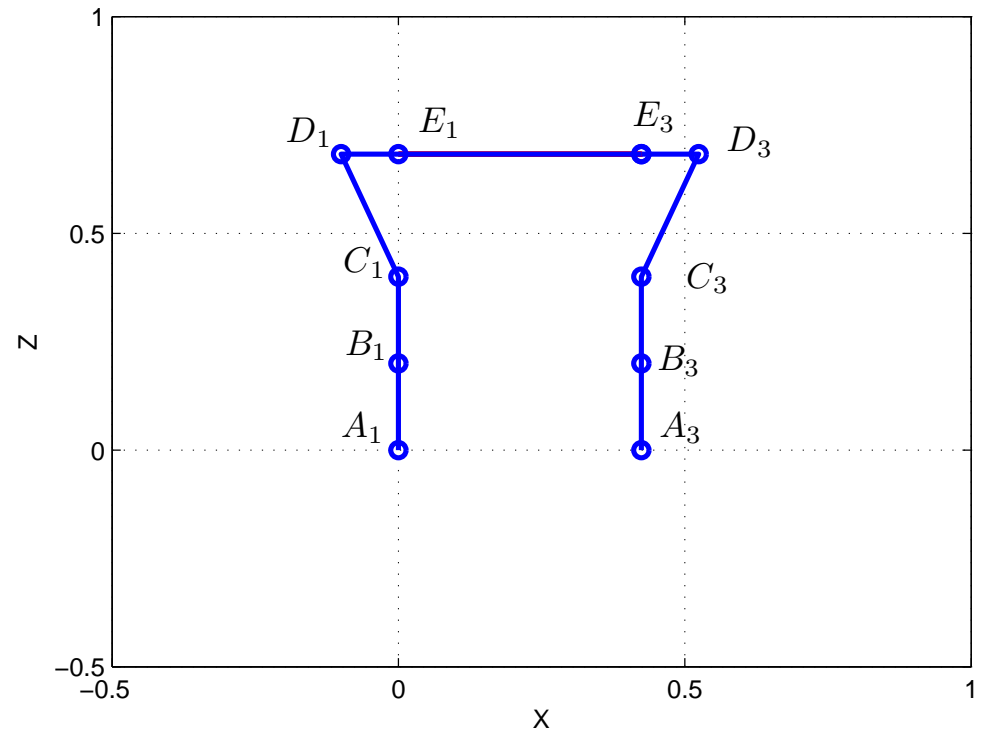

Figura 3.3: Segundo caso de singularidade. 
Em ambos os casos, a singularidade ocorre dentro do espaço de trabalho da plataforma, mas não existe problema de travamento do sistema já que, se existirem mais juntas ativas, bastará qualquer movimento infinitesimal destas juntas para tirar o mecanismo da condição de singularidade.

\subsubsection{Singularidade direta da plataforma}

O segundo tipo de singularidade ocorre quando $\operatorname{det}\left(J_{x}\right)=0$. No nosso caso, a matriz, $J_{x}$ é igual ao produto das matrizes $J_{x_{1}}$ e $J_{x_{2}}$. Logo, se o $\operatorname{det}\left(J_{x_{1}}\right)=0$ ou $\operatorname{det}\left(J_{x_{2}}\right)=0$ está-se diante de uma singularidade da cinemática direta.

De (3.13) temos que os valores singulares de $J_{x_{2}}$ acontecem quando $\theta_{P}=$ $0, \pi, \ldots, n \pi \forall n \in \mathbb{N}$. Esta condição de singularidade depende da convenção dos ângulos de Euler escolhida na Figura 2.4. Para uma convenção do tipo $Z^{\prime}-Y^{\prime}-$ $Z^{\prime \prime}$ a condição de singularidade $\theta_{P}=0, \pi, \ldots, n \pi$ aconteceria para toda posição horizontal da plataforma, o que seria inadmissível. Além disto, um valor tal de $\theta_{P}$ provocaria a degeneração do sistema (2.12). Para contornar este problema, podese mudar a convenção escolhida dos ângulos de Euler ou mudar a orientação do sistema de referência $\{O\}$ (Harib e Srinivasan, 2003a). Mudando a orientação de $\{O\}$ como mostra a Figura 3.4 fazemos com que agora a singularidade aconteça

para $\theta_{P}=\frac{\pi}{2}, \frac{3 \pi}{2} \ldots, \frac{(2 n+1) \pi}{2} \forall n \in \mathbb{N}$ o que significaria uma posição da plataforma completamente na vertical com relação à superfície de apoio (posição praticamente improvável). Como nosso robô foi projetado para fins de movimentos na vertical, esta mudança de orientação não afeta o raciocínio seguido neste trabalho (Figura $3.4)$.

A matriz $J_{x_{1}}$ pode se transformar numa matriz quadrada para determinadas condições de trabalho, por exemplo, quando o robô está fixo à superfície por duas pernas, enquanto as outras duas estão no ar. Neste caso a matriz $J_{x_{1}}$ teria dimensão $6 \times 6$, podendo causar singularidade ao sistema quando $\operatorname{det}\left(\Omega_{F_{j}}-\Omega_{F_{k}}\right)=0$ para $j, k=1,2,3,4: j \neq k$. 


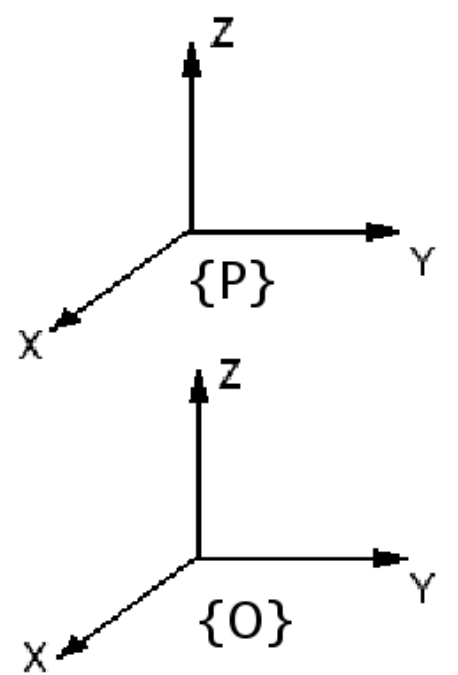

(a) $\theta_{\mathrm{p}}=0$

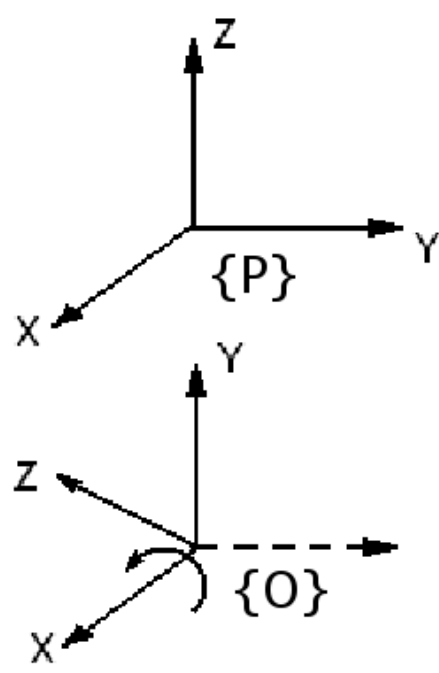

(b) $\theta_{p}=-\pi / 2$

Figura 3.4: Condição de singularidade para $\theta_{P}=0$ (a) e $\theta_{P}=-\frac{\pi}{2}(\mathrm{~b})$

Neste tipo de singularidade pode existir qualquer movimento infinitesimal da plataforma, embora os atuadores de cada perna estejam completamente bloqueados, perdendo esta sua rigidez completamente. Diz-se então que a plataforma ganha um ou mais graus de liberdade. Este fato constitui uma contradição com os manipuladores seriais, que na presença de singularidades perdem um ou mais graus de liberdade.

\subsubsection{Singularidade combinada da plataforma}

O último tipo de singularidade seria uma combinação das duas primeiras. Esta singularidade somente pode acontecer para determinadas características físicas de projeto do robô e sob a influência de certas configurações. Matematicamente seria que $\operatorname{det}\left(J_{q}\right)=0$ e $\operatorname{det}\left(J_{x}\right)=0$ ao mesmo tempo. Portanto, o sistema ganharia alguns graus de liberdade com nenhum movimento dos atuadores, enquanto perderia também alguns graus de liberdade para determinados movimentos destes. 


\subsubsection{Aceleração da plataforma}

Para terminar a análise cinemática feita nesta seção basta calcular os vetores aceleração angular da plataforma e aceleração linear do ponto ${ }^{O} P$ em relação ao sistema $\{O\}$.

Derivando (3.6) com relação ao tempo obtemos a aceleração angular.

$$
\left[\begin{array}{c}
o \dot{\omega}_{x} \\
o \dot{\omega}_{y} \\
o \dot{\omega}_{z}
\end{array}\right]=\left[\begin{array}{ccc}
0 & -s \phi_{P} & s \theta_{P} c \phi_{P} \\
0 & c \phi_{P} & s \theta_{P} s \phi_{P} \\
1 & 0 & c \theta_{P}
\end{array}\right]\left[\begin{array}{c}
\ddot{\phi}_{P} \\
\ddot{\theta}_{P} \\
\ddot{\psi}_{P}
\end{array}\right]+\left[\begin{array}{ccc}
0 & -c \phi_{P} \dot{\phi}_{P} & \dot{\theta}_{P} c \theta_{P}-\dot{\psi_{P}} s \psi_{P} \\
0 & s \phi_{P} \dot{\phi}_{P} & \dot{\theta}_{P} c \theta_{P}-\dot{\psi}_{P} c \psi_{P} \\
0 & 0 & -\dot{\theta}_{P} s \theta_{P}
\end{array}\right]\left[\begin{array}{c}
\dot{\phi}_{P} \\
\dot{\theta}_{P} \\
\dot{\psi}_{P}
\end{array}\right]
$$

Já para obter a aceleração linear, podemos calcular a derivada com relação ao tempo da equação (3.14) diretamente.

$$
\dot{J}_{q} \overrightarrow{\dot{q}}+J_{q} \overrightarrow{\ddot{q}}=\dot{J}_{x} \overrightarrow{\dot{x}}+J_{x} \overrightarrow{\ddot{x}}
$$

onde

$$
\dot{J}_{x}=\dot{J}_{x_{1}} J_{x_{2}}+J_{x_{1}} \dot{J}_{x_{2}} .
$$

As matrizes $\dot{J}_{q}, \dot{J}_{x_{1}}$ e $\dot{J}_{x_{2}}$ são obtidas pela diferenciação de $J_{q}, J_{x_{1}}$ e $J_{x_{2}}$ com relação ao tempo. Logo:

$$
\dot{J}_{q}=\left[\begin{array}{lll}
a_{11} & a_{12} & a_{13} \\
a_{21} & a_{22} & a_{23} \\
a_{31} & a_{32} & a_{33}
\end{array}\right]
$$

onde:

$$
\begin{aligned}
& a_{11}=-c \theta_{4_{l}} \dot{\theta}_{4_{l}}\left(c \theta_{2_{l} 3_{l}} L_{2}+c \theta_{3_{l}} L_{3}\right)-s \theta_{4_{l}}\left(-s \theta_{2_{l} 3_{l}}\left(\dot{\theta}_{3_{l}}+\dot{\theta}_{2_{l}}\right) L_{2}-s \theta_{3_{l}} \dot{\theta}_{3_{l}} L_{3}\right), \\
& a_{12}=s \theta_{4_{l}} \dot{\theta}_{4_{l}}\left(s \theta_{2_{l} 3_{l}} L_{2}+s \theta_{3_{l}} L_{3}\right)-c \theta_{4_{l}}\left(-c \theta_{2_{l} 3_{l}}\left(\dot{\theta}_{3_{l}}+\dot{\theta}_{2_{l}}\right) L_{2}-c \theta_{3_{l}} \dot{\theta}_{3_{l}} L_{3}\right), \\
& a_{13}=s \theta_{4_{l}} \dot{\theta} L_{2} s \theta_{2_{l} 3_{l}}-c \theta_{4_{l}} L_{2} c \theta_{2_{l} 3_{l}}\left(\dot{\theta}_{3_{l}}+\dot{\theta}_{2_{l}}\right), \\
& a_{21}=-s \theta_{4_{l}} \dot{\theta}_{4_{l}}\left(c \theta_{2_{l} 3_{l}} L_{2}+c \theta_{3_{l}} L_{3}\right)+c \theta_{4_{l}}\left(-s \theta_{2_{l} 3_{l}}\left(\dot{\theta}_{3_{l}}+\dot{\theta}_{2_{l}}\right) L_{2}-s \theta_{3_{l}} \dot{\theta}_{3_{l}} L_{3}\right), \\
& a_{22}=-c \theta_{4_{l}} \dot{\theta}_{4_{l}}\left(s \theta_{2_{l} 3_{l}} L_{2}+s \theta_{3_{l}} L_{3}\right)-s \theta_{4_{l}}\left(-c \theta_{2_{l} 3_{l}}\left(\dot{\theta}_{3_{l}}+\dot{\theta}_{2_{l}}\right) L_{2}-c \theta_{3_{l}} \dot{\theta}_{3_{l}} L_{3}\right), \\
& a_{23}=-c \theta_{4_{l}} \dot{\theta}_{2} s \theta_{2_{l} 3_{l}}-s \theta_{4_{l}} L_{2} c \theta_{2_{l} 3_{l}}\left(\dot{\theta}_{3_{l}}+\dot{\theta}_{2_{l}}\right),
\end{aligned}
$$




$$
\begin{aligned}
& a_{31}=0, \\
& a_{32}=-s \theta_{2_{l} 3_{l}}\left(\dot{\theta}_{3_{l}}+\dot{\theta}_{2_{l}}\right) L_{2}-s \theta_{3_{l}} \dot{\theta}_{3_{l}} L_{3}, \\
& a_{33}=-s \theta_{2_{l} 3_{l}}\left(\dot{\theta}_{3_{l}}+\dot{\theta}_{2_{l}}\right) L_{2} .
\end{aligned}
$$

Finalmente,

$$
\dot{J}_{x_{1}}=\left[\begin{array}{cc}
0_{3 \times 3} & \dot{\Omega}_{F_{1}} \\
\vdots & \vdots \\
0_{3 \times 3} & \dot{\Omega}_{F_{4}}
\end{array}\right]
$$

e

$$
\dot{J}_{x_{2}}=\left[\begin{array}{c}
0_{3 \times 3} \\
0_{3 \times 3}
\end{array} \quad\left[\begin{array}{ccc}
0 & -c \phi_{P} \dot{\phi}_{P} & \dot{\theta}_{3 \times 3} c \theta_{P}-\dot{\psi}_{P} s \psi_{P} \\
0 & s \phi_{P} \dot{\phi}_{P} & \dot{\theta}_{P} c \theta_{P}-\dot{\psi}_{P} c \psi_{P} \\
0 & 0 & -\dot{\theta}_{P} s \theta_{P}
\end{array}\right]\right]
$$

\subsection{Análise cinemática de velocidade da garra}

De forma similar a como foi feita a análise cinemática de velocidade da plataforma, a análise cinemática de velocidade da perna se reduz ao cálculo da matriz jacobiana que relaciona o movimento das juntas da perna $l$ com as coordenadas cartesianas de seu efetuador, neste caso, a garra (ponto $A_{l}$ ).

\subsubsection{Singularidade da garra}

O estudo das singularidades da perna $l$ é equivalente à análise das singularidades de um manipulador serial com base no ponto $E_{l}$. Diferentemente da análise da plataforma, em que o sistema podia ganhar ou perder graus de liberdade, quando ocorre uma condição de singularidade na perna, esta só pode perder graus de liberdade.

A equação (3.31) mostra a relação entre as velocidades das juntas da perna $l$ com as velocidades da garra em relação ao sistema $\left\{E_{l}\right\}$.

$$
\vec{v}_{A_{l}}=J_{A_{l}} \dot{\overrightarrow{q_{l}}}
$$


onde:

$$
\dot{\vec{x}}_{A_{l}}=\vec{v}_{A_{l}}=\left[\begin{array}{c}
E_{l} v_{A_{x_{l}}} \\
E_{l} v_{A_{y_{l}}} \\
E_{l} v_{A_{z_{l}}} \\
\dot{\psi}_{l}
\end{array}\right]
$$

Para calcular $J_{A_{l}}$ é necessário diferenciar (2.20) com relação ao tempo e adicionar uma linha à matriz correspondente ao ângulo de rotação da garra $\psi_{l}=\theta_{4_{l}}$ :

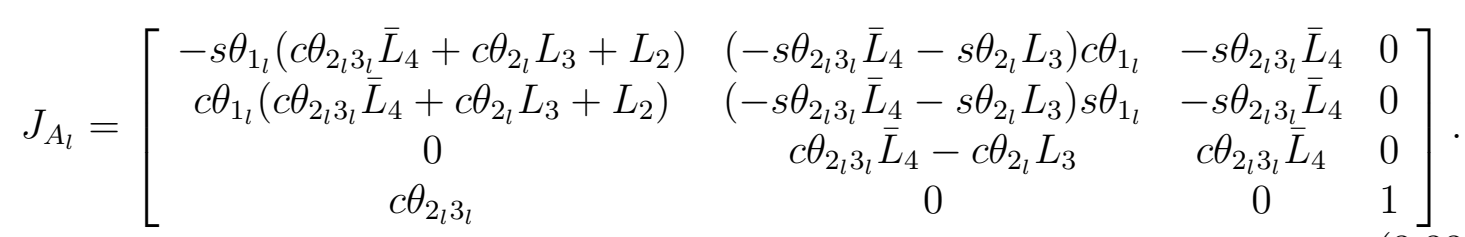

Neste caso, a singularidade da matriz $J_{A_{l}}$ se manifesta para $\operatorname{det}\left(J_{A_{l}}\right)=0$ ou de forma equivalente para:

$$
c \theta_{2_{l} 3_{l}} \bar{L}_{4}+c \theta_{2_{l}} L_{3}+L_{2}=0
$$

$\mathrm{ou}$

$$
\left(-s \theta_{2_{l}{ }_{3}} \bar{L}_{4}-s \theta_{2_{l}} L_{3}\right) c \theta_{2_{l} 3_{l}}-\left(s \theta_{1}+c \theta_{1_{l}}\right)\left(c \theta_{2_{l} 3_{l}} \bar{L}_{4}+c \theta_{2_{l}} L_{3}\right) s \theta_{2_{l} 3_{l}}=0 .
$$

A primeira configuração (3.34) se dá para:

$$
s \theta_{2_{l}}=\frac{s \theta_{3} \bar{L}_{4} L_{2} \pm\left(c \theta_{3_{l}} \bar{L}_{4}+L_{3}\right) \sqrt{\bar{L}_{4}^{2}+2 c \theta_{3_{l}} L_{3} \bar{L}_{4}+L_{3}^{2}-L_{2}^{2}}}{\bar{L}_{4}^{2}+2 c \theta_{3_{l}} L_{3} \bar{L}_{4}+L_{3}^{2}}
$$

onde:

$$
c \theta_{3_{l}} \geq \frac{L_{2}^{2}-L_{3}^{2}-\bar{L}_{4}^{2}}{2 L_{3} \bar{L}_{4}}
$$

Note que esta desigualdade garante também que o denominador de (3.36) seja não nulo.

Semelhantemente ao problema abordado no estudo da plataforma, as singularidades mostradas nas Figuras 3.5 e 3.5 ocorrem no interior do espaço de trabalho do manipulador, porém, como todas as juntas da perna são ativas, não existe risco de 


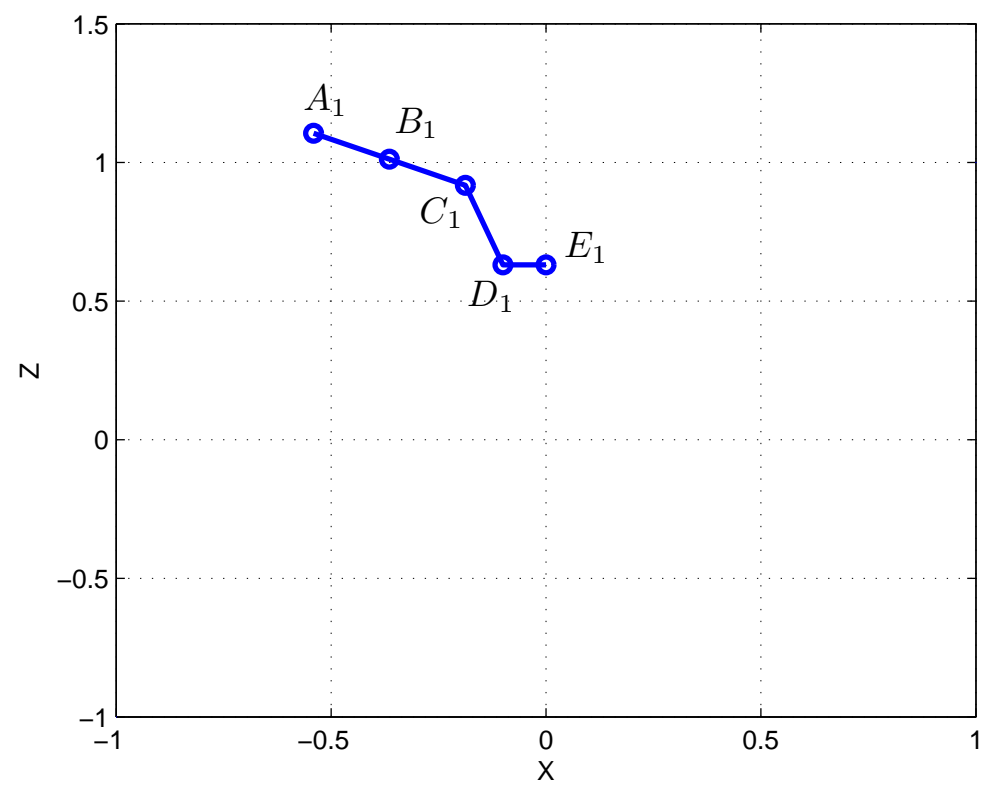

Figura 3.5: Exemplo de singularidade da perna 1 no ar para $\theta_{2_{1}}=1.274$ e $\theta_{3_{1}}=-\frac{\pi}{4}$.

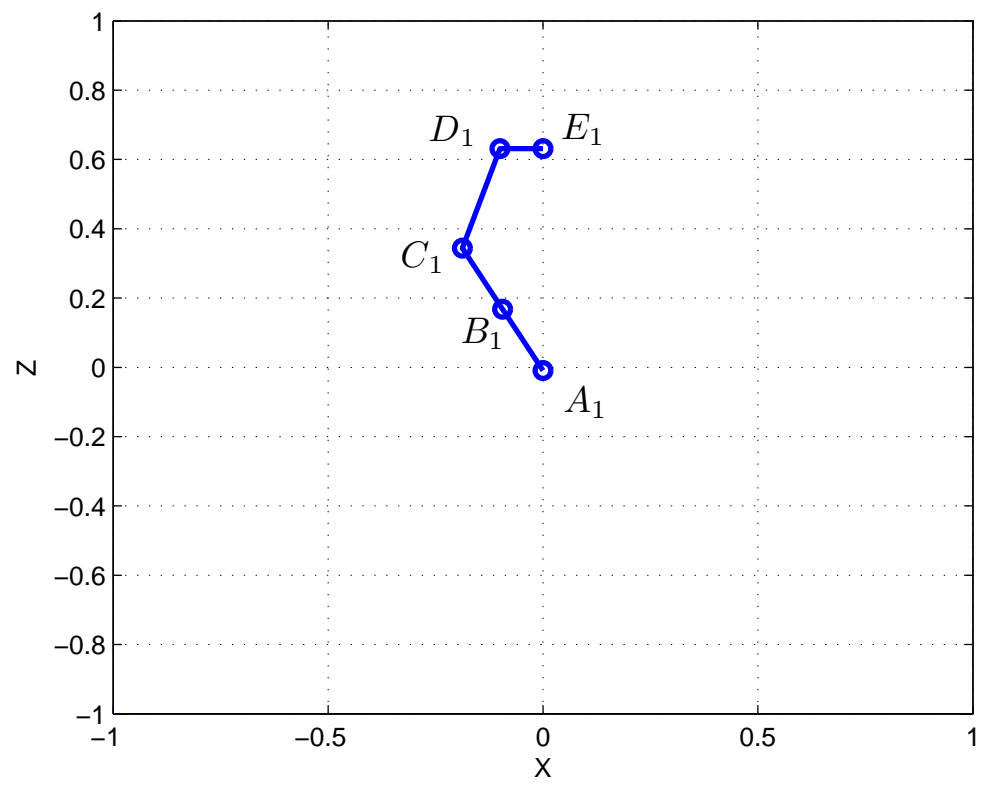

Figura 3.6: Exemplo de singularidade da perna 1 no ar para $\theta_{2_{1}}=-1.274$ e $\theta_{3_{1}}=$ $-\frac{\pi}{4}$

travamento. Este fato é devido a que qualquer movimento infinitesimal das demais juntas forçaria o sistema a sair da sua condição de singularidade.

Outros exemplos de singularidades da perna, porém, na fronteira do espaço de 
trabalho, ocorrem de acordo com (3.35). Nas Figuras 3.7, 3.8 e 3.9 são apresentados três exemplos destas configurações singulares.

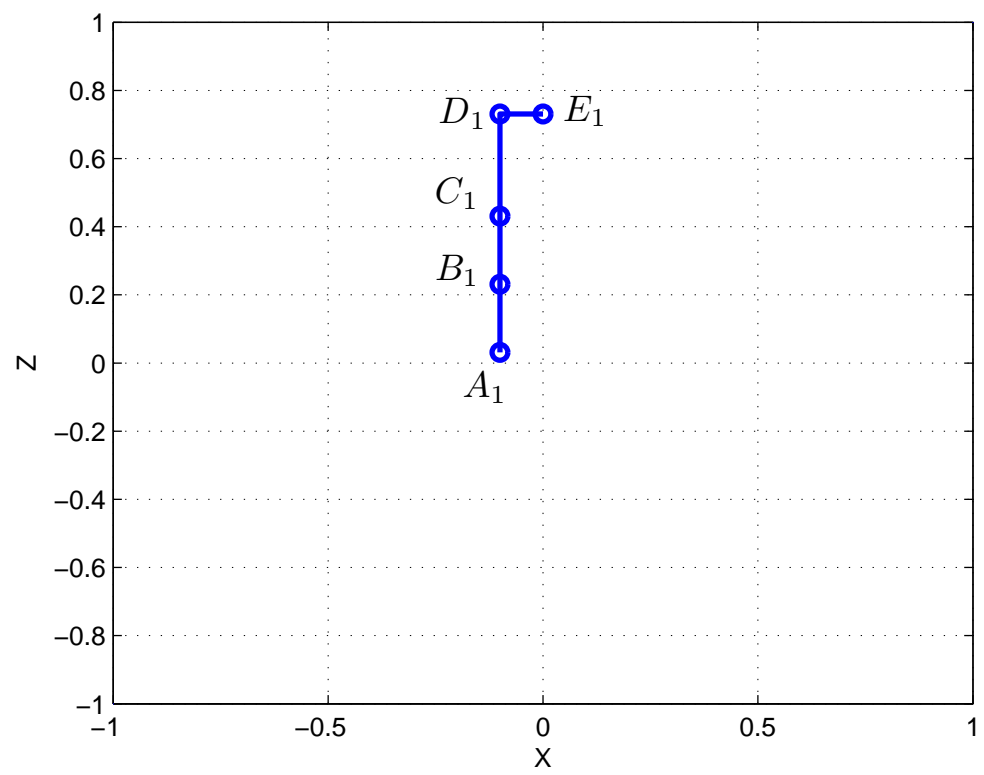

Figura 3.7: Singularidade na fronteira do espaço de trabalho da perna 1 no ar. $\left(\theta_{2_{1}}=-\frac{\pi}{2}, \theta_{3_{1}}=0\right)$.

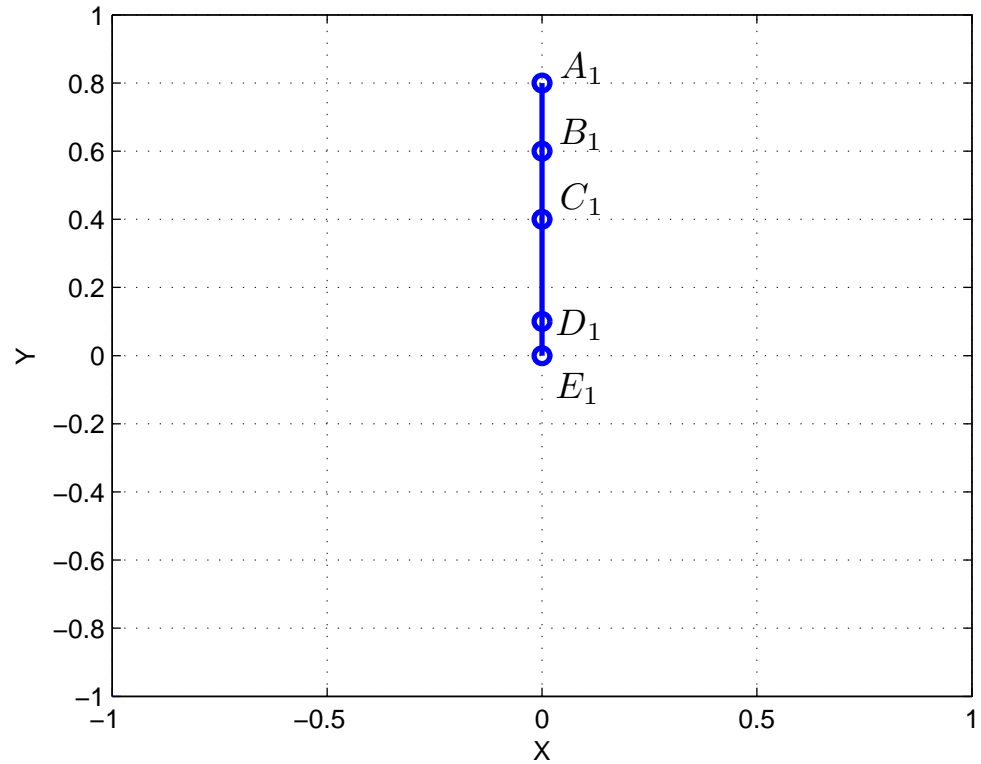

Figura 3.8: Singularidade na fronteira do espaço de trabalho da perna 1 no ar. $\left(\theta_{1_{1}}=\frac{\pi}{2}, \theta_{2_{1}}=\theta_{3_{1}}=0\right)$. 


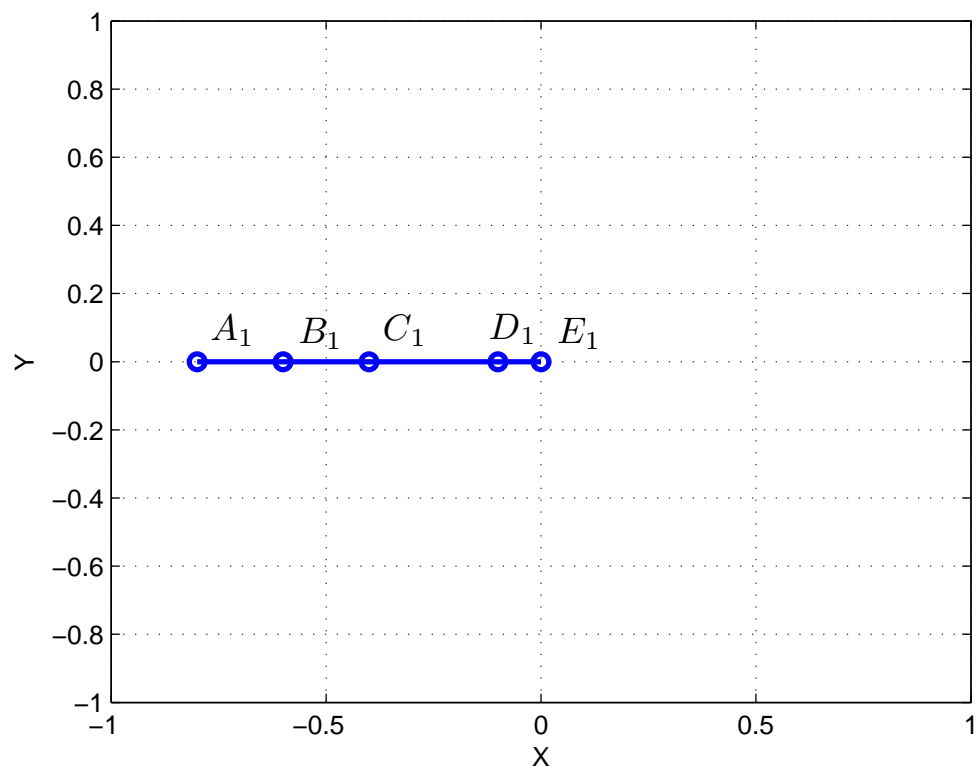

Figura 3.9: Singularidade na fronteira do espaço de trabalho da perna 1 no ar. $\left(\theta_{1_{1}}=\pi, \theta_{2_{1}}=\theta_{3_{1}}=0\right)$.

\subsubsection{Aceleração da garra}

Para finalizar a análise da perna basta calcular a aceleração da garra em relação ao sistema $\left\{E_{l}\right\}$. Similarmente ao que foi feito no caso da plataforma, devemos derivar (3.31) com relação ao tempo.

$$
\dot{\vec{v}}_{A_{l}}=\dot{J}_{A_{l}} \cdot E_{l} \dot{\overrightarrow{q_{l}}}+J_{A_{l}} \cdot E_{l} \ddot{\overrightarrow{q_{l}}}
$$

onde

$$
\dot{J}_{A_{l}}=\left[\begin{array}{llll}
b_{11} & b_{12} & b_{13} & b_{14} \\
b_{21} & b_{22} & b_{23} & b_{23} \\
b_{31} & b_{32} & b_{33} & b_{34} \\
b_{41} & b_{42} & b_{43} & b_{44}
\end{array}\right]
$$

$\mathrm{e}$

$$
\begin{aligned}
& b_{11}=-c \theta_{1_{l}} \dot{\theta}_{1_{l}}\left(c \theta_{2_{l} 3_{l}} \bar{L}_{4}+c \theta_{2_{l}} L_{3}+L_{2}\right)-s \theta_{1_{l}}\left(-s \theta_{2_{l} 3_{l}}\left(\dot{\theta}_{2_{l}}+\dot{\theta}_{3_{l}}\right) \bar{L}_{4}-s \theta_{2_{l}} \dot{\theta}_{2_{l}} L_{3}\right), \\
& b_{21}=-s \theta_{1_{l}} \dot{\theta}_{1_{l}}\left(c \theta_{2_{l} 3_{l}} \bar{L}_{4}+c \theta_{2_{l}} L_{3}+L_{2}\right)+c \theta_{1_{l}}\left(-s \theta_{2_{l} 3_{l}}\left(\dot{\theta}_{2_{l}}+\dot{\theta}_{3_{l}}\right) \bar{L}_{4}-s \theta_{2_{l}} \dot{\theta}_{2_{l}} L_{3}\right), \\
& b_{31}=0
\end{aligned}
$$




$$
\begin{aligned}
& b_{41}=0, \\
& b_{12}=-s \theta_{1_{l}} \dot{\theta}_{1_{l}}\left(-s \theta_{2_{l} 3_{l}} \bar{L}_{4}-s \theta_{2_{l}} L_{3}\right)-c \theta_{1_{l}}\left(c \theta_{2_{l} 3_{l}}\left(\dot{\theta}_{2_{l}}+\dot{\theta}_{3_{l}}\right) \bar{L}_{4}-c \theta_{2_{l}} \dot{\theta}_{2_{l}} L_{3}\right), \\
& b_{22}=c \theta_{1_{l}} \dot{\theta}_{1_{l}}\left(-s \theta_{2_{l} 3_{l}} \bar{L}_{4}-s \theta_{2_{l}} L_{3}\right)+s \theta_{1_{l}}\left(c \theta_{2_{l} 3_{l}}\left(\dot{\theta}_{2_{l}}+\dot{\theta}_{3_{l}}\right) \bar{L}_{4}-c \theta_{2_{l}} \dot{\theta}_{2_{l}} L_{3}\right), \\
& b_{32}=-s \theta_{2_{l} 3_{l}}\left(\dot{\theta}_{2_{l}}+\dot{\theta}_{3_{l}}\right) \bar{L}_{4}+s \theta_{2_{l}} \dot{\theta}_{2_{l}} L_{3}, \\
& b_{42}=0, \\
& b_{13}=-c \theta_{2_{l} 3_{l}}\left(\dot{\theta}_{2_{l}}+\dot{\theta}_{3_{l}}\right) \bar{L}_{4}, \\
& b_{23}=-c \theta_{2_{l} 3_{l}}\left(\dot{\theta}_{2_{l}}+\dot{\theta}_{3_{l}}\right) \bar{L}_{4}, \\
& b_{33}=-s \theta_{2_{l} 3_{l}}\left(\dot{\theta}_{2_{l}}+\dot{\theta}_{3_{l}}\right) \bar{L}_{4}, \\
& b_{43}=0, \\
& b_{14}=-s \theta_{2_{l} 3_{l}}\left(\dot{\theta}_{2_{l}}+\dot{\theta}_{3_{l}}\right), \\
& b_{24}=0, \\
& b_{34}=0 \\
& b_{44}=0 .
\end{aligned}
$$




\section{Capítulo 4}

\section{Dinâmica}

\subsection{Introdução}

Analisar a dinâmica de um sistema significa encontrar a relação que existe entre o seu movimento e as forças e/ou torques aplicados neste. No caso de um robô quadrúpede sua dinâmica é muito mais complexa devido à variação periódica da topologia do sistema. Esta variação ocorre toda vez que cadeias cinemáticas abrem ou fecham, como por exemplo quando uma perna do robô é liberada de seu ponto de apoio e fica no ar ou quando duas ou mais pernas suportam o peso do robô e movem a plataforma numa direção (Pfeiffer et al., 1995a).

Existem varias técnicas para calcular a dinâmica de robôs. Entre elas podemos citar a formulação Lagrangiana, o método de Newton-Euler, o método de Kane e o Principio do Trabalho Virtual ou de d'Alembert (Lin, 1990; Tsai, 1999; Santana, 2005; Choi et al., 2004; Tartari e Cabral, 2006; Merlet, 2006).

Diferentemente da formulação Lagrangiana, que trata o robô como um todo e utiliza o conceito de energia através da equação de Lagrange, a formulação de Newton-Euler considera cada ligamento do robô em separado, escrevendo suas equações de movimento linear e angular. Como cada ligamento se encontra unido aos demais através de juntas, cada equação de movimento é afetada pela ação de forças e torques resultantes do movimento dos outros ligamentos, desta forma todas as equações 
estão acopladas umas às outras. Logo, por meio de um processo recursivo é possível resolver o conjunto de equações que modelam o robô como um todo.

Embora a formulação Lagrangiana trabalhe com menos equações que o método de Newton-Euler, a presença de numerosas restrições nas cadeias fechadas de um robô paralelo tornam quase impossível a obtenção de equações explícitas de movimentos em função de variáveis independentes. Uma solução intermediária poderia ser a utilização do princípio do Trabalho Virtual ou de d'Alembert. Este método trabalha com menos equações que o de Newton-Euler e consegue equacionar de forma eficiente a dinâmica de um robô paralelo, porém a dificuldade dele está na formulação das chamadas matrizes do manipulador e dos ligamentos (Tsai, 1999). Finalmente as equações utilizadas pelo método de Kane são deduzidas a partir do produto escalar das equações obtidas pelo método de Newton-Euler e pelas derivadas parciais das velocidades lineares e angulares dos centros de gravidade de cada ligamento em relação às variáveis de movimento. A quantidade final de equações normalmente coincide com os graus de liberdade do mecanismo mas tem-se a dificuldade da determinação das variáveis de movimento (Kane e Levinson, 1985; Xu et al., 1999; Finotti, 2008).

Para a análise dinâmica da plataforma será utilizado o Princípio do Trabalho Virtual ou de d'Alembert e, para a análise dinâmica da perna, o método iterativo de Newton-Euler. Em ambos os casos, foi considerado que as massas dos ligamentos se encontram concentras em seu centro de massa. Esta abordagem é muito mais simples de formular e ao mesmo tempo tem demonstrado ser suficientemente exata na modelagem de sistemas dinâmicos complexos com movimentos lentos, como foi mostrado em Almeida e Hess-Coelho (2010).

Antes de começar a trabalhar com as equações dinâmicas será necessário conhecer primeiramente as velocidades e acelerações de cada ligamento.

Neste capítulo será utilizada a notação usada em Tsai (1999).

- $f_{i_{l}}$ : força resultante, excluindo a força do atuador, exercida sobre o centro de 
massa do ligamento $i_{l}$.

- $f_{i_{l}}^{*}$ : força de inércia exercida sobre o centro de massa do ligamento $i_{l}$.

- $f_{i_{l}}^{*}=-m_{i} \dot{\overrightarrow{\vec{v}}}_{i_{l}}$.

- $\hat{f}_{i_{l}}=f_{i_{l}}+f_{i_{l}}^{*}$.

- $f_{p}$ : força resultante exercida sobre o centro de massa da plataforma.

- $f_{p}^{*}$ : força de inércia exercida sobre o centro de massa da plataforma.

- $f_{p}^{*}=-m_{p} \dot{\overrightarrow{v_{p}}}$.

- $\hat{f}_{p}=f_{p}+f_{p}^{*}$.

- $n_{i_{l}}$ : torque resultante, excluindo o torque do atuador, exercido em relação ao centro de massa do ligamento $i_{l}$.

- $n_{i_{l}}^{*}$ : torque de inércia exercido sobre o centro de massa do ligamento $i_{l}$.

- $n_{i_{l}}^{*}=-{ }^{i_{l}} I_{i_{l}} \dot{\overrightarrow{\omega_{i_{l}}}}-{ }^{i_{l}} \vec{\omega}_{i_{l}} \times\left({ }^{i_{l}} I_{i_{l}} \overrightarrow{\omega_{l}}\right)$.

- $\hat{n}_{i_{l}}=n_{i_{l}}+n_{i_{l}}^{*}$.

- $n_{p}$ : torque resultante exercido sobre o centro de massa da plataforma.

- $n_{p}^{*}$ : torque de inércia exercido em relação ao centro de massa da plataforma.

- $n_{p}^{*}=-I_{p} \dot{\overrightarrow{\omega_{p}}}-\vec{\omega}_{p} \times\left(I_{p} \overrightarrow{\omega_{p}}\right)$.

- $\hat{n}_{p}=n_{p}+n_{p}^{*}$.

- $\delta(\cdot)$ : deslocamento virtual de $(\cdot)$.

Além disto, por conveniência serão introduzidos os vetores:

$$
\hat{F}_{i}=\left[\begin{array}{c}
\hat{f}_{i} \\
\hat{n}_{i}
\end{array}\right]
$$


e

$$
\hat{F}_{p}=\left[\begin{array}{c}
\hat{f}_{p} \\
\hat{n}_{p}
\end{array}\right]
$$

para representar a soma das forças e torques aplicados sobre os ligamentos e a plataforma respectivamente.

\subsection{Acelerações dos ligamentos}

Para calcular as forças que agem nos ligamentos é necessário obter as expressões da aceleração linear do centro de massa e das velocidades e acelerações angulares em cada instante de tempo.

Este processo é realizado seguindo uma lógica iterativa que começa na base da perna do robô e avança até seu extremo. A base e o extremo da perna podem mudar de acordo com o sistema de referência utilizado.

\subsubsection{Aceleração angular}

Do capítulo anterior são conhecidas a velocidade e a aceleração angular de cada junta. Nosso objetivo agora é calcular as grandezas cinemáticas de cada ligamento do robô.

Começando pela base da perna do robô, ponto $E_{l}$ da Figura 2.3, temos:

$$
{ }^{(i+1)_{l}} \omega_{(i+1)_{l}}={ }^{(i+1)_{l}} R_{i_{l}} \cdot{ }^{i_{l}} \omega_{i_{l}}+\dot{\theta}_{(i+1)_{l}} \cdot{ }^{(i+1)_{l}} \hat{Z}_{(i+1)_{l}}
$$

onde $^{(i+1)_{l}} \hat{Z}_{(i+1)_{l}}$ é o versor do eixo da junta expresso no sistema $\left\{(i+1)_{l}\right\},{ }^{(i+1)_{l}} R_{i_{l}}$ a matriz de rotação do sistema $\left\{i_{l}\right\}$ com relação ao sistema $\left\{(i+1)_{l}\right\}$ e ${ }^{(i+1)_{l}} \omega_{(i+1)_{l}}$ a velocidade angular da junta $(i+1)_{l}$, para $i=1,2,3,4$ e $l=1,2,3,4$.

Esta equação caracteriza a velocidade angular do ligamento $(i+1)_{l}$. Já para 
obter a aceleração do ligamento em questão bastaria derivar (4.1) em relação ao tempo:

$$
{ }^{(i+1)_{l}} \dot{\omega}_{(i+1)_{l}}={ }^{(i+1)_{l}} R_{i_{l}} \cdot{ }^{i_{l}} \dot{\omega}_{i_{l}}+\ddot{\theta}_{(i+1)_{l}} \cdot{ }^{(i+1)_{l}} \hat{Z}_{(i+1)_{l}}+{ }^{(i+1)_{l}} R_{i_{l}} \cdot{ }^{i_{l}} \omega_{i_{l}} \times \dot{\theta}_{(i+1)_{l}} \cdot{ }^{(i+1)_{l}} \hat{Z}_{(i+1)_{l}} .
$$

\subsubsection{Aceleração linear}

A velocidade linear da origem do sistema $\left\{(i+1)_{l}\right\}$ pode ser calculada pela equação:

$$
{ }^{(i+1)_{l}} v_{(i+1)_{l}}={ }^{(i+1)_{l}} R_{i_{l}}\left({ }^{i_{l}} v_{i_{l}}+{ }^{i_{l}} \omega_{i_{l}} \times{ }^{i_{l}} 0_{(i+1)_{l}}\right)
$$

onde ${ }^{i_{l}} 0_{(i+1)_{l}}$ é a posição da origem do sistema $\left\{(i+1)_{l}\right\}$ preso ao ligamento $(i+1)_{l}$ com relação ao sistema $\left\{i_{l}\right\}$ (Figura 2.2 ) para $i=1,2,3,4$ e $l=1,2,3,4$.

A aceleração é obtida derivando as equações (4.3) em relação ao tempo:

$$
{ }^{(i+1)_{l}} \dot{v}_{(i+1)_{l}}={ }^{(i+1)_{l}} R_{i_{l}}\left[{ }^{i} \dot{\omega}_{i} \times{ }^{i_{l}} 0_{(i+1)_{l}}+{ }^{i_{l}} \omega_{i_{l}} \times\left({ }^{i_{l}} \omega_{i_{l}} \times{ }^{i_{l}} 0_{(i+1)_{l}}\right)+{ }^{i_{l}} \dot{v}_{i_{l}}\right]
$$

Para levar em conta o efeito da gravidade sobre a perna basta fazer ${ }^{O} \dot{v}_{O}=-{ }^{O} g$ onde ${ }^{O} g$ é o vetor aceleração da gravidade expresso no sistema $O,{ }^{O} g=\left[\begin{array}{lll}0 & -g & 0\end{array}\right]$.

\subsection{Matriz de inércia de cada ligamento}

Um corpo rígido no espaço pode girar em torno de infinitos eixos de rotação. Baseando-se neste principio, cada ligamento do Kamambaré poderia girar em torno de mais de um eixo de rotação, porém como eles encontram-se enlaçados uns aos outros, seus eixos de rotação não podem ser arbitrários.

Cada perna do Kamambaré possui 4 ligamentos unidos através de 4 juntas rotacionais e cada junta tem somente um eixo de rotação. Assim, em relação aos 
ligamentos vizinhos, cada ligamento poderá girar no máximo em torno dos eixos de rotação das juntas de seus extremos.

O tensor de inércia de cada ligamento relativo a um sistema $\{(x, y, z)\}$ fixo ao próprio corpo pode ser expresso na forma de uma matriz de dimensões $3 \times 3$

$$
I=\left[\begin{array}{ccc}
I_{x x} & -I_{x y} & -I_{x z} \\
-I_{x y} & I_{y y} & -I_{y z} \\
-I_{x z} & -I_{y z} & I_{z z}
\end{array}\right]
$$

onde:

$$
\begin{gathered}
I_{x x}=\iiint_{V}\left(y^{2}+z^{2}\right) \rho_{c} d V_{c}, \\
I_{x y}=\iiint_{V}(x y) \rho_{c} d V_{c}
\end{gathered}
$$

e assim por diante. Aqui $\rho_{c}$ é a densidade do corpo e $V_{c}$ seu volume. Os termos $I_{x x}, I_{y y}$ e $I_{z z}$ são chamados momentos de inércia de massa enquanto $I_{x y}, I_{y z}$ e $I_{x z}$ são chamados produtos de inércia de massa. No caso da plataforma, ela pode ser considerada como uma placa retangular de massa $m_{p}$ e dimensões $a \times b$ girando em torno de seu eixo $Z_{P}$ (Figura 4.1).

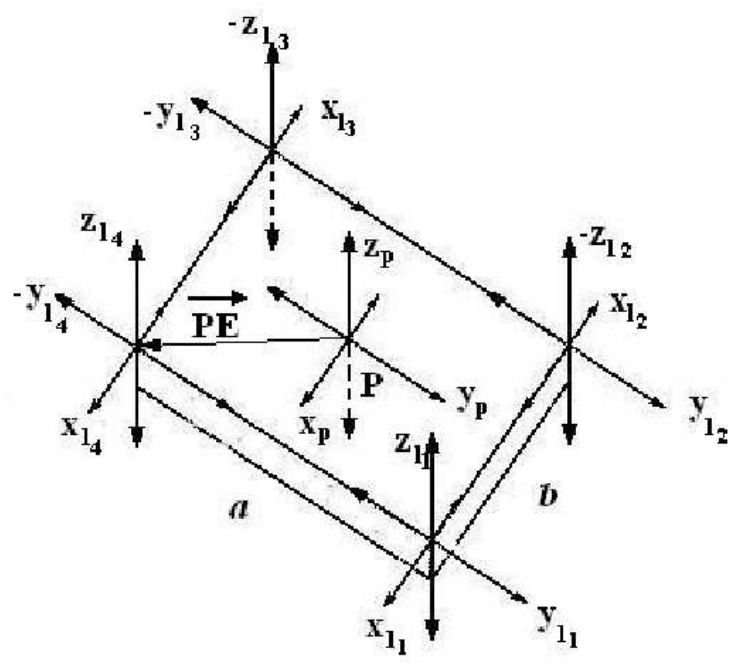

Figura 4.1: Eixos de rotação da plataforma 


\subsection{Análise dinâmica da plataforma}

O princípio d'Alembert ou do trabalho virtual afirma que "é nulo o trabalho realizado ao longo de um deslocamento virtual infinitesimal arbitrário de um sistema, a partir de uma posição de equilíbrio" (Leech, 1971). Este princípio é mais geral que o princípio de Hamilton pois evita a restrição a sistemas holonômicos (sistemas cujos vínculos dependem somente das coordenadas e do tempo, e não das velocidades). Se os termos negativos nas acelerações são encarados como forças inerciais, o princípio de d'Alembert pode ser formulado então no sentido do trabalho virtual como: O trabalho virtual total realizado pelas forças aplicadas mais as forças inerciais é zero para deslocamentos reversíveis.

Assim o princípio do trabalho virtual pode ser apresentado no caso do Kamambaré como:

$$
\delta \vec{q}^{T} \vec{\tau}+\delta \vec{x}_{p}^{T} \hat{F}_{p}+\sum_{l=1}^{\tilde{L}} \sum_{i=2}^{4}\left(\delta \vec{x}_{i_{l}}^{T} \hat{F}_{i_{l}}\right)=0
$$

onde $\vec{\tau}$ é o vetor de torques formado por $\tau_{i_{l}} \forall i=2, \ldots, 4 ; l=1, \ldots, \tilde{L}$ e $\tilde{L}=2,3$ ou 4 equivale ao número de pernas que estão fixas à superfície de apoio suportando o peso do robô.

O deslocamento virtual de (4.8) tem de ser compatível com as restrições geométricas e cinemáticas do sistema. Logo é necessário referenciar este deslocamento virtual a um conjunto de deslocamentos virtuais gerais independentes. Para isto, as coordenadas de posição e orientação da plataforma $\vec{x}_{p}=\left[P_{x}, P_{y}, P_{z}, \phi_{p}, \theta_{p}, \psi_{p}\right]$ podem ser convenientemente escolhidas como coordenadas generalizadas. Esta transformação é possível devido a que o deslocamento virtual das juntas $\delta \vec{q}$, está relacionado ao deslocamento virtual da plataforma $\delta \vec{x}_{p}$, por meio da matriz jacobiana $J_{p}$ 


$$
\delta \vec{q}=J_{p} \delta \vec{x}_{p}
$$

onde $\delta \vec{q}$ é o deslocamento virtual da plataforma e $J_{p}=J_{q}^{-1} J_{x}$.

Da mesma forma, o deslocamento virtual dos ligamentos pode também ser relacionado com o deslocamento virtual da plataforma:

$$
\delta \vec{x}_{i_{l}}=J_{i_{l}} \delta \vec{x}_{p} .
$$

Substituindo (4.9) e (4.10) em (4.8) obtém-se:

$$
\delta \vec{x}_{p}\left(J_{p}^{T} \vec{\tau}+\hat{F}_{p}+\sum_{l=1}^{\tilde{L}} \sum_{i=2}^{4}\left(J_{i_{l}}^{T} \hat{F}_{i_{l}}\right)\right)=0
$$

e, como (4.11) é valida para qualquer deslocamento virtual $\delta \vec{x}_{p}$,

$$
J_{p}^{T} \vec{\tau}+\hat{F}_{p}+\sum_{l=1}^{\tilde{L}} \sum_{i=2}^{4}\left(J_{i_{l}}^{T} \hat{F}_{i_{l}}\right)=0
$$

Se o número de atuadores é igual ao número de graus de liberdade do sistema, a matriz $J_{p}$ é quadrada e portanto $\vec{\tau}$ está unicamente determinado; porém, como o número de atuadores é maior que o número de graus de liberdade do robô, há um número infinito de soluções para $\vec{\tau}$. Assim, uma solução de norma mínima pode ser adotada mediante a aplicação da matriz pseudo-inversa de $J_{p}^{T}$ :

$$
\vec{\tau}=-J_{p}^{-T}\left(\hat{F}_{p}+\sum_{l=1}^{\tilde{L}} \sum_{i=2}^{4}\left(J_{i_{l}}^{T} \hat{F}_{i_{l}}\right)\right) .
$$

Para a aplicação deste método, primeiramente serão calculadas as velocidades lineares e angulares de cada ligamento, em seguida as matrices jacobianas dos ligamentos e por último as forças e torques exercidas nos ligamentos.

Para o cálculo das matrizes jacobianas dos ligamentos serão utilizadas as equações (4.1) e (4.3), mas aqui as equações são re-arranjadas nas formas: 


$$
{ }^{i_{l}} \omega_{i_{l}}={ }^{i_{l}} R_{(i+1)_{l}}\left({ }^{(i+1)_{l}} \omega_{(i+1)_{l}}-\dot{\theta}_{(i+1)_{l}} \cdot{ }^{(i+1)_{l}} \hat{Z}_{(i+1)_{l}}\right)
$$

e

$$
{ }^{i_{l}} v_{i_{l}}={ }^{i_{l}} R_{(i+1)_{l}} \cdot{ }^{(i+1)_{l}} v_{(i+1)_{l}}-{ }^{i} \omega_{i} \times{ }^{i} 0_{(i+1)_{l}}
$$

para $i=1,2, \ldots, 3$ e $l$ sendo igual ao número de pernas que suportam o peso da plataforma nesse momento.

Assume-se que o processo iterativo começa na plataforma $(i=1)$ com os vetores ${ }^{(i+1)_{l}} \omega_{(i+1)_{l}}=\left[\omega_{p_{x}}, \omega_{p_{y}}, \omega_{p_{z}}\right] \mathrm{e}^{(i+1)_{l}} v_{(i+1)_{l}}=\left[v_{p_{x}}, v_{p_{y}}, v_{p_{z}}\right]$ e vai até o ligamento 4. Uma vez resolvidas as equações (4.14) e (4.15) é possível calcular as matrizes jacobianas dos ligamentos. Estes cálculos são mostrados nos Apêndices A e B, respectivamente.

Finalmente, para o cálculo das forças que atuam na plataforma e nos ligamentos, somente é tomada em consideração a ação da força da gravidade. Assim:

$$
\hat{F}_{p}=\left[\begin{array}{c}
m_{p} \vec{g}-m_{p} \dot{\vec{v}}_{p} \\
-{ }^{O} I_{p} \dot{\vec{\omega}}_{p}-\omega_{p} \times\left({ }^{O} I_{p} \vec{\omega}_{p}\right)
\end{array}\right]
$$

e

$$
\hat{F}_{i_{l}}=\left[\begin{array}{c}
m_{i_{l}} \vec{g}-m_{i_{l}} \dot{\vec{v}}_{i_{l}} \\
-{ }^{O} I_{i_{l}} \dot{\vec{\omega}}_{i_{l}}-\omega_{i_{l}} \times\left({ }^{O} I_{i_{l}} \vec{\omega}_{i_{l}}\right)
\end{array}\right]
$$

para $i=1,2, \ldots, 3$ e $l$ sendo igual ao número de pernas que suportam o peso da plataforma nesse momento.

Logo com todas estas equações é possível resolver (4.13).

\subsection{Análise dinâmica da perna}

Para a análise dinâmica da perna foi escolhido o método de Newton-Euler. Este método utiliza na sua formulação todas as forças atuantes em cada um dos ligamentos. Portanto, as equações dinâmicas obtidas incluem todas as forças, momentos e restrições entre os ligamentos adjacentes. Estas restrições são muito úteis 
na hora de dimensionar as juntas e os ligamentos. Na sua implementação o método pode ser resumido em duas etapas principais:

1. Na primeira etapa, as velocidades e acelerações dos ligamentos são calculadas da base até o extremo do mecanismo e as equações de Newton-Euler são aplicadas a cada ligamento. Nesta etapa serão utilizadas as equações (4.1), (4.2), (4.3), (4.4), (4.19) e (4.20).

2. Numa segunda etapa as forças e torques de interação e os esforços nas juntas são calculados, começando no extremo e avançando até a base do mecanismo.

Assim para começar, suponha-se que a posição do centro de massa ${ }^{(i+1)_{l}} O_{C_{(i+1)_{l}}}$ de cada ligamento $(i+1)_{l}$ é conhecida em relação ao sistema $\left\{(i+1)_{l}\right\}$ do ligamento. Então é possível calcular sua aceleração através da seguinte equação:

$$
\begin{aligned}
{ }^{(i+1)_{l}} \dot{v}_{c_{(i+1)_{l}}=}{ }^{(i+1)_{l}} \dot{\omega}_{(i+1)_{l}} \times{ }^{(i+1)_{l}} O_{C_{(i+1)_{l}}}+ \\
{ }^{(i+1)_{l}} \omega_{(i+1)_{l}} \times\left({ }^{(i+1)_{l}} \omega_{(i+1)_{l}} \times{ }^{(i+1)_{l}} O_{C_{(i+1)_{l}}}\right)+{ }^{(i+1)_{l}} \dot{v}_{(i+1)_{l}}
\end{aligned}
$$

Considerando que o ligamento $(i+1)_{l}$ é um corpo rígido de massa $m_{(i+1)_{l}}$ e que seu centro de massa se desloca com aceleração ${ }^{(i+1)_{l}} \dot{v}_{c_{(i+1)_{l}}}$ sob a ação de uma força resultante ${ }^{(i+1)_{l}} f_{(i+1)_{l}}^{*}$ nele aplicada, a equação de Newton para ele pode ser escrita como:

$$
{ }^{(i+1)_{l}} f_{(i+1)_{l}}^{*}=m_{(i+1)_{l}}{ }^{(i+1)_{l}} \dot{v}_{c_{(i+1)_{l}}} .
$$

Se, além do deslocamento, o ligamento $(i+1)_{l}$ também gira com uma velocidade angular ${ }^{(i+1)_{l}} \omega_{(i+1)_{l}}$ e aceleração angular ${ }^{(i+1)_{l}} \dot{\omega}_{(i+1)_{l}}$ sob a ação de um momento resultante ${ }^{(i+1)_{l}} n_{(i+1)_{l}}^{*}$ em relação ao seu centro de massa, a equação de Euler pode 
ser escrita como:

$$
{ }^{(i+1)_{l}} n_{(i+1)_{l}}^{*}={ }^{C_{(i+1)_{l}}} I_{(i+1)_{l}} \cdot{ }^{(i+1)_{l}} \dot{\omega}_{(i+1)_{l}}+{ }^{(i+1)_{l}} \omega_{(i+1)_{l}} \times\left({ }^{C_{(i+1)}} I_{(i+1)_{l}} \cdot{ }^{(i+1)_{l}} \dot{\omega}_{\left.(i+1)_{l}\right)}\right) .
$$

Na equação (4.20) o sistema $C_{(i+1)_{l}}$ está fixo ao ligamento $(i+1)_{l}$ e é tal que sua origem se localiza no centro de massa do ligamento. O parâmetro ${ }^{(i+1)_{l}} \omega_{(i+1)_{l}}$ representa a velocidade angular do corpo em relação a um sistema inercial, mas expressa em $C_{(i+1)_{l}}$. Já o termo ${ }^{(i+1)_{l}} \omega_{(i+1)_{l}} \times\left({ }^{C_{(i+1)_{l}}} I_{(i+1)_{l}} \cdot{ }^{(i+1)_{l}} \dot{\omega}_{(i+1)_{l}}\right)$ é chamado de giroscópico.

Uma das vantagens de escrever a equação de Euler no sistema fixo ao ligamento é que desta forma a matriz de inércia é constante. Num sistema inercial, como por exemplo, o sistema $\{O\}$, esta matriz varia com o tempo (Spong e Vidyasagar, 1995).

Cada ligamento do robô está sujeito à ação de forças e torques exercidos sobre ele por seus vizinhos, além da força e torques inerciais resultantes de seu movimento (Craig, 1989; Khan et al., 2005).

Na forma de recorrências e avançando desde a junta 3 até a junta 1 as equações a serem resolvidas para $i=3,2, \ldots, 1$ são:

$$
{ }^{i_{l}} \hat{f}_{i_{l}}={ }^{i_{l}} R_{(i+1)_{l}} \cdot{ }^{(i+1)_{l}} f_{(i+1)_{l}}+{ }^{i_{l}} f_{i_{l}}^{*}
$$

e

$$
{ }^{i_{l}} \hat{n}_{i_{l}}={ }^{i_{l}} n_{i_{l}}^{*}+{ }^{i_{l}} R_{(i+1)_{l}}{ }^{(i+1)_{l}} n_{(i+1)_{l}}+{ }^{i_{l}} 0_{c_{i_{l}}} \times{ }^{i_{l}} f_{i_{l}}^{*}+{ }^{i_{l}} 0_{(i+1)_{l}} \times{ }^{i_{l}} R_{(i+1)_{l}} \cdot{ }^{(i+1)_{l}} f_{(i+1)_{l}}
$$

onde, como a perna não está sujeita a nenhuma força externa,

$$
{ }^{n+1} f_{n+1}=0
$$

e

$$
{ }^{n+1} n_{n+1}=0 .
$$




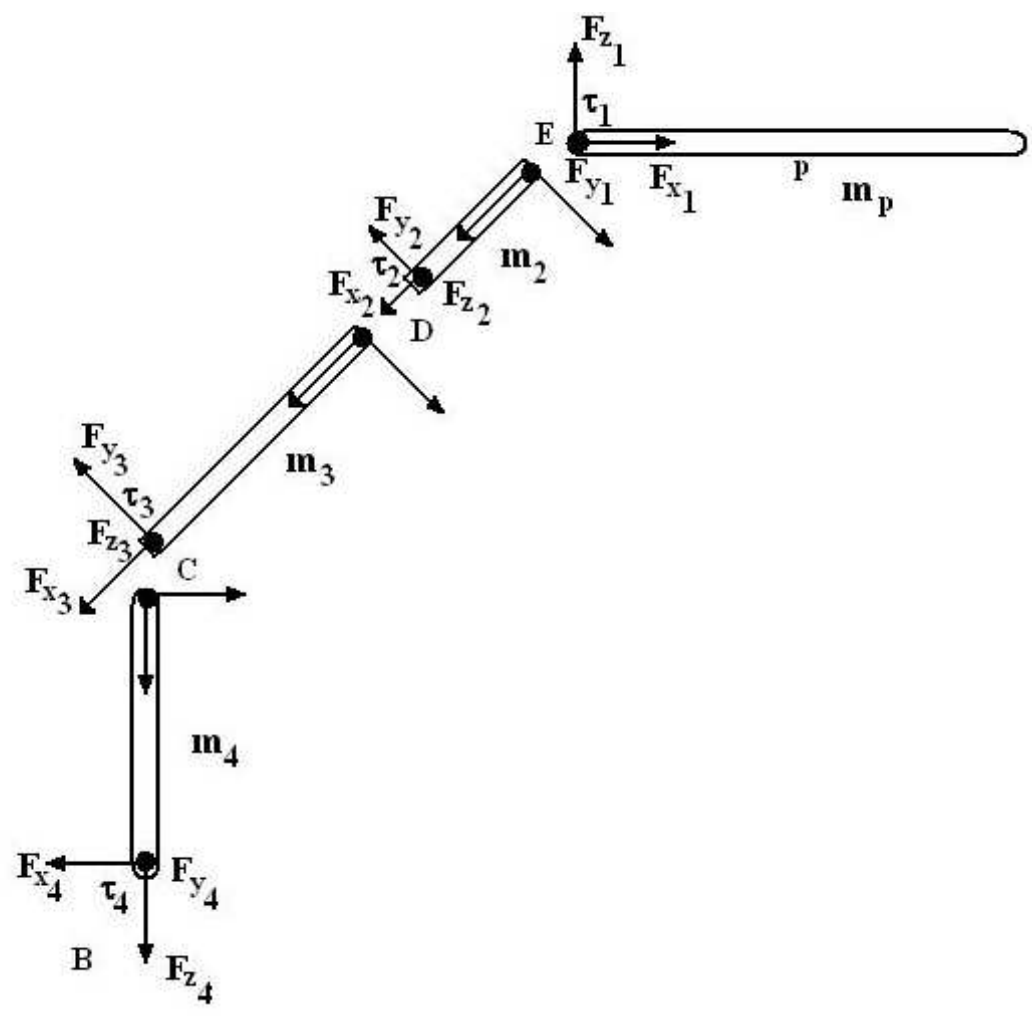

Figura 4.2: Esquema de forças e torques para uma perna do Kamambaré

Finalmente para juntas rotacionais temos:

$$
\tau_{i_{l}}={ }^{i_{l}} \hat{n}_{i_{l}}^{T} \cdot{ }^{i_{l}} \hat{Z}_{i_{l}}
$$

onde $\tau_{i_{l}}$ é o torque do atuador da junta $i_{l}$.

Embora o método de Newton-Euler considere um grande número de equações de forças intermediárias que não são utilizadas no controle, sua escolha é fundamentada pela sua fácil implementação devida à sua natureza recursiva.

\subsection{Efeitos não modelados}

As soluções obtidas não levam em conta todos os efeitos que agem sobre as juntas e os ligamentos. Elas consideram apenas a dinâmica do corpo rígido sob a ação da gravidade. Uma força muito importante que não foi incluída no modelo é a 
força de atrito.

Cada junta do Kamambaré está sujeita a reduções de engrenagens, nestas circunstâncias os efeitos do atrito podem chegar a representar $25 \%$ do torque necessário para acionar uma junta em situações típicas (Craig, 1989).

Os efeitos do atrito viscoso e de Coulomb são modelados de forma simplificada pela equação:

$$
\tau_{\text {atrito }_{i_{l}}}=\beta_{i_{l}} \cdot \operatorname{sgn}\left(\dot{\theta}_{i_{l}}\right)+\gamma_{i_{l}} \cdot \dot{\theta}_{i_{l}}
$$

para cada uma das juntas, onde $\beta_{i_{l}}$ e $\gamma_{i_{l}}$ são constantes.

Outro efeito desconsiderado na análise feita é, por exemplo, a flexibilidade dos ligamentos, porém, para fins de controle, os modelos obtidos representam com boa fidelidade as características dinâmicas do robô. 


\section{Capítulo 5}

\section{Algoritmo de Otimização}

\subsection{Introdução}

O planejamento de trajetórias ou OFMPP pode ser considerado como um problema clássico na teoria de controle ótimo. Este problema pode ser resolvido utilizando métodos numéricos específicos que alguns autores classificam em diretos e indiretos (Stryk e Bulirsch, 1992; Hull, 1997; Betts, 1998). Os métodos indiretos são baseados no cálculo de variações ou no Principio do Máximo de Pontryagin. Estes geralmente levam a problemas não-lineares de múltiplos valores de fronteira que podem ser resolvidos utilizando técnicas apropriadas, como por exemplo o método de múltiplos disparos, e precisam de um bom critério de partida. Infelizmente a aplicação deles está acompanhada de um grande conhecimento da natureza física e matemática do sistema a otimizar (Chettibi et al., 2005).

Diferentemente dos métodos indiretos, os diretos são baseados em transformações do sistema original em problemas de programação não-linear por meio da discretização de algumas ou de todas as variáveis dinâmicas do sistema. Esta nova forma de abordagem tem-se mostrado robusta e simples em muitas aplicações.

A aplicação de técnicas de controle ótimo a robôs de cadeia fechada, tais como, manipuladores, robôs antropomórficos e robôs paralelos em geral é uma técnica relativamente recente, isto é devido à complexidade do seu movimento, à grande 
quantidade de ligamentos e corpos que interagem no mecanismo e à dificuldade de fazer uma modelagem exata do sistema. Além disto alguns autores como Stryk e Bulirsch (1992) têm observado a dificuldade de calcular o espaço de estado do sistema, uma vez que o conjunto de equações não-lineares sujeitas às restrições inerentes do sistema têm de ser resolvidas em cada iteração do processo, na busca da solução final.

Neste capítulo será feita a apresentação de um problema de otimização de trajetórias para robôs, visando minimizar as perdas elétricas dos motores ativos em cada perna. Supõem-se conhecidas a cinemática e a dinâmica do robô. A abordagem será feita pelo método direto através da transformação do problema original em um de programação quadrática sujeito a restrições lineares.

\subsection{Modelo do motor}

Existem duas técnicas principais para o controle de cadeias cinemáticas, o controle independente por junta e o controle multivariável. Neste último enfoque todas as equações dinâmicas da cadeia cinemática formam um complexo sistema nãolinear multivariável. Deve-se ter em vista também, que este esquema de controle é fortemente baseado no modelo, portanto abre questões a respeito da sua robustez. Ou seja, a implementação prática dele requer um bom conhecimento do modelo e da estrutura do sistema que normalmente estão sujeitas a incertezas tais como, cargas desconhecidas, atrito seco nos eixos, ocorrência de folgas e flexibilidades não modeladas.

Diferentemente do controle multivariável, no controle independente por junta cada ligamento é modelado como um sistema SISO (única entrada/unica saída), onde os efeitos de acoplamento dos demais ligamentos sobre a junta são considerados como perturbações no sistema, e os efeitos das incertezas mencionadas anteriormente são minimizados pela relação de redução de engrenagem (Spong e Vidyasagar, 1995). 
Cada junta do robô é controlada por um motor de corrente continua (C.C.) através de uma redução por engrenagens. A Figura 5.2 mostra o esquema geral de um motor de C.C. acionando uma carga mecânica.

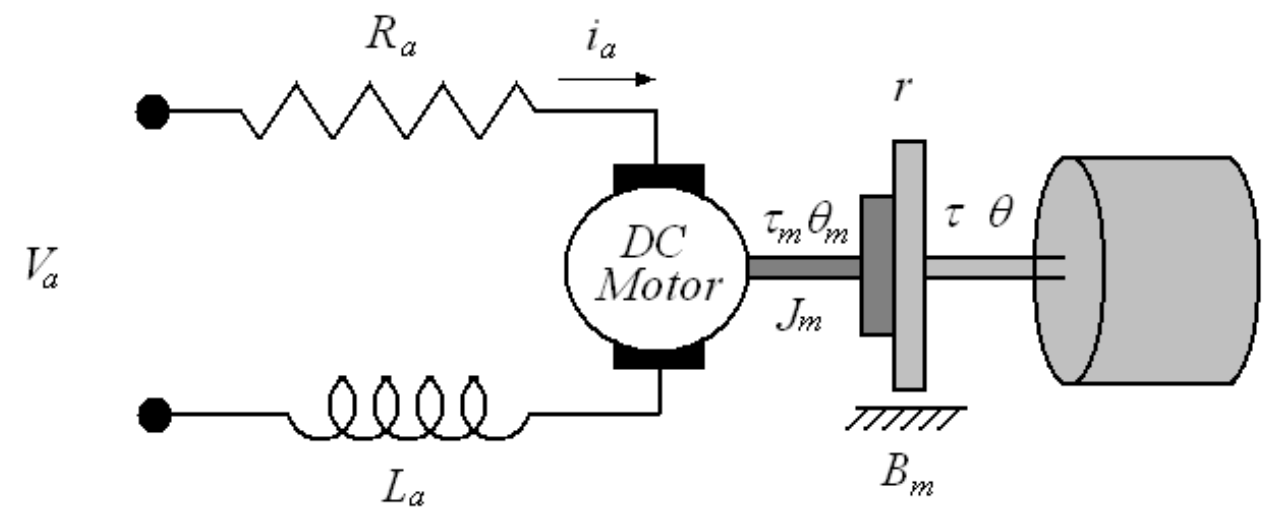

Figura 5.1: Diagrama eletro-mecânico de uma junta.

Para melhor entender o funcionamento do motor C.C., este pode ser dividido em três subsistemas: o magnético, o elétrico e o mecânico.

O subsistema magnético pode ser descrito pelas seguintes equações:

$$
\chi_{m}(t)=K_{e} i_{e}(t)
$$

e

$$
\tau_{m}(t)=K_{p} i_{a}(t) \chi_{m}(t)
$$

onde $i_{e}(t)$ é a corrente de excitação, $i_{a}(t)$ é a corrente de armadura, $\chi_{m}(t)$ é o fluxo gerado pela corrente de excitação, $K_{e}$ e $K_{p}$ são constantes e $\tau_{m}$ é o torque do motor.

Como a corrente de excitação pode ser assumida constante, a equação (5.2) é reescrita como:

$$
\tau_{m}(t)=K_{m} i_{a}(t)
$$

onde $K_{m}$ é a constante de torque do motor. 
Devido ao fluxo magnético $\chi_{m}(t)$ existe uma força eletromotriz que induz uma tensão proporcional à velocidade angular do motor. Esta tensão é representada por:

$$
V_{m}(t)=K_{b} \dot{\theta}_{m}(t)
$$

onde $\theta_{m}(t)$ é a posição angular do rotor e $K_{b}$ é a constante de força contraeletromotriz do motor.

O subsistema elétrico é definido por:

$$
V_{a}(t)=R_{a} i_{a}(t)+L_{a} \dot{i}_{a}(t)+V_{m}(t)
$$

Em (5.5), $V_{a}, R_{a}, L_{a}$ e $i_{a}$ são a tensão, resistência, indutância e corrente de armadura do motor.

Por fim, o subsistema mecânico é representado pela equação:

$$
\tau_{m}(t)=J_{m} \ddot{\theta}_{m}(t)+B_{m} \dot{\theta}_{m}(t)+r \tau(t)
$$

onde $r$ é o factor de redução por engrenagem, $J_{m}$ representa a inércia do rotor, $B_{m}$ é o coeficiente de amortecimento viscoso do motor e $\tau$ é o torque da carga.

Como a constante de tempo do subsistema mecânico (5.6) é muito maior que a constante de tempo do subsistema eléctrico (5.5), a indutância do motor $L_{a}$ pode ser descartada sem perda de exatidão do modelo.

Finalmente, usando as equações (5.2), (5.5) e (5.6), o problema é reformulado como:

$$
\begin{gathered}
J_{m} \ddot{\theta}_{m}(t)+B_{m} \dot{\theta}_{m}(t)=K_{m} i_{a}(t)-r \tau(t), \\
V_{a}(t)=R_{a} i_{a}(t)+K_{b} \dot{\theta}_{m}(t)
\end{gathered}
$$

$\mathrm{e}$

$$
\theta(t)=r \theta_{m}(t)
$$




\subsubsection{Controle independente por junta}

Como já foi mencionado na seção anterior, no controle independente por junta todos os efeitos de acoplamento devidos ao movimento dos demais ligamentos são ignorados ou tratados como perturbações do sistema (Spong e Vidyasagar, 1995). Assim, o ponto-chave da abordagem consiste no fato de $\tau$, resultante do movimento dos outros ligamentos, ser tratado como uma perturbação e ser atenuado pela multiplicação do fator redutor $r$, o qual é geralmente muito menor do que 1 (Figura 5.2). Desta forma, a redução por engrenagem reduz o efeito e transmissão das não-linearidades presentes na dinâmica das pernas do robô. Além disto, o controle utilizado será feito manipulando o torque gerado pelo motor.

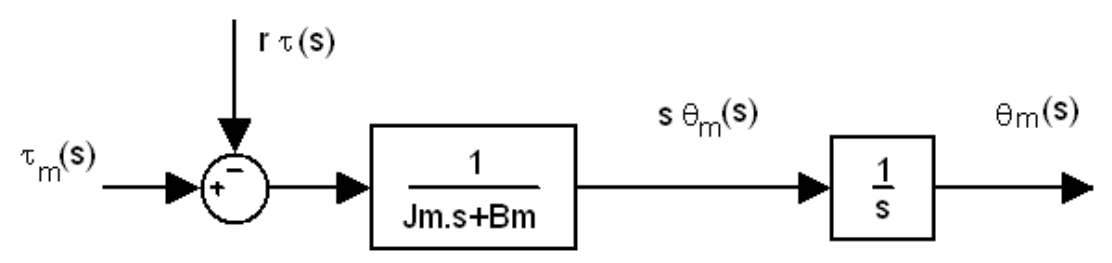

Figura 5.2: Controle independente por junta, utilizando como variável manipulada o torque do motor.

Assume-se que trabalhamos num sistema onde existem $L$ cadeias cinemáticas, com $M$ juntas cada, todas elas controladas por motores de C.C. Logo os índices $l$ e $i$, definidos em capítulos precedentes, serão usados de agora em diante nas equações (5.1) até (5.9) para indicar a i-ésima junta da l-ésima cadeia cinemática.

Para escrever o sistema no espaço de estados, é preciso definir os dois estados básicos do sistema (5.7) posição e velocidade angular do rotor:

$$
x_{m_{i_{l}}}=\left[\begin{array}{c}
\theta_{m_{i_{l}}} \\
\dot{\theta}_{m_{i_{l}}}
\end{array}\right] \text {. }
$$

Então a equação que modela a dinâmica do robô na forma de espaço de estados 
para a $i$-ésima junta e $(1 \leq i \leq M)$ da l-ésima cadeia cinemática ou perna do robô $(1 \leq l \leq L)$ é dada pela equação (5.11)

$$
\left\{\begin{array}{l}
\dot{x}_{m_{i_{l}}}=\bar{A}_{i_{l}} x_{m_{i_{l}}}+\bar{B}_{i_{l}} u_{i_{l}} \\
y_{m_{i_{l}}}=\bar{C}_{i_{l}} x_{m_{i_{l}}}
\end{array}\right.
$$

onde:

$$
\begin{gathered}
u_{i_{l}}=\tau_{m_{i_{l}}}-r_{i_{l}} \tau_{i_{l}}, \\
\bar{A}_{i_{l}}=\left[\begin{array}{cc}
0 & 1 \\
0 & -\frac{B_{m_{i_{l}}}}{J_{m_{i_{l}}}}
\end{array}\right], \\
\bar{B}_{i_{l}}=\left[\begin{array}{c}
0 \\
\frac{1}{J_{m_{i_{l}}}}
\end{array}\right],
\end{gathered}
$$

$\mathrm{e}$

$$
\bar{C}_{i_{l}}=\left[\begin{array}{ll}
1 & 0
\end{array}\right]
$$

O vetor de torques $\tau$ é usualmente expresso na forma da equação de espaço de configuração:

$$
\tau=\mathbf{M}_{\mathbf{l}}\left[\ddot{\theta}_{i_{l}}\right]+\mathbf{B}_{\mathbf{l}}\left[\dot{\theta}_{x_{l}} \dot{\theta}_{y_{l}}\right]+\mathbf{C}_{\mathbf{l}}\left[\dot{\theta}_{i_{l}}^{2}\right]+\mathbf{G}_{\mathbf{l}}
$$

onde $\mathbf{M}_{\mathbf{l}} \in \mathbb{R}^{M \times M}, \mathbf{M}_{\mathbf{l}}=\mathbf{M}_{\mathbf{l}}^{T}>0$ é a matriz de massa da cadeia cinemática, $\mathbf{B}_{\mathbf{l}} \in \mathbb{R}^{M \times M}$ é a matriz de coeficientes de Coriolis, $\left[\dot{\theta}_{x_{l}} \dot{\theta}_{y_{l}}\right]$ é o vetor produto definido como $\left[\dot{\theta}_{x_{l}} \dot{\theta}_{y_{l}}\right] \forall x_{l} \neq y_{l}, \mathbf{C}_{\mathbf{l}} \in \mathbb{R}^{M \times M}$ é a matriz dos coeficientes centrífugos, $\left[\dot{\theta}_{i_{l}}^{2}\right] \forall i$ : $1 \leq i \leq M$ e $\mathbf{G}_{\mathbf{l}} \in \mathbb{R}^{M}$ é vetor de torques de gravidade, todos referentes à perna $l$.

Como nossa intenção consiste em minimizar a potência consumida por cada motor, o índice de desempenho ou função objetivo adotada são as perdas elétricas ou de Joule nas armaduras dos motores:

$$
\varepsilon_{i_{l}}=\min _{u_{i_{l}}} \int_{0}^{t_{f}} P_{i_{l}} d t
$$


o problema anterior está sujeito às seguintes restrições:

$$
\begin{gathered}
{\left[\begin{array}{c}
\theta_{m_{i_{l}}}\left(t_{f}\right) \\
\dot{\theta}_{m_{i_{l}}}\left(t_{f}\right)
\end{array}\right]=\left[\begin{array}{c}
\tilde{\theta}_{m_{i_{l}}} \\
0
\end{array}\right]} \\
\left|i_{a_{i_{l}}}(t)\right| \leq I_{\text {max }_{i_{l}}} \forall t \in\left[0, t_{f}\right] \\
\left|V_{a_{i_{l}}}(t)\right| \leq V_{\max _{i_{l}}} \forall t \in\left[0, t_{f}\right] \\
\check{\theta}_{m_{i_{l}}} \leq \theta_{m_{i_{l}}}(t) \leq \hat{\theta}_{m_{i_{l}}} \forall t \in\left[0, t_{f}\right]
\end{gathered}
$$

onde $P_{i_{l}}$ representa a potência instantânea do motor dissipada na $i$-ésima junta da l-ésima cadeia cinemática.

$$
P_{i_{l}}(t)=R_{a_{i_{l}}} i_{a_{i_{l}}}^{2}(t)
$$

$\mathrm{e}$

$$
i_{a_{i_{l}}}(t)=\frac{1}{K_{m_{i_{l}}}} u_{i_{l}}(t)-\frac{r_{i_{l}}}{K_{m_{i_{l}}}} \tau_{i_{l}}(t) .
$$

Nestas equações, $t_{f}$ é o tempo necessário para deslocar o robô ou uma perna dele desde uma posição inicial até uma final, $I_{\max _{i_{l}}}$ é a máxima corrente de armadura, a tensão máxima aplicável é $V_{\max _{i_{l}}}$, os limites mínimo e máximo do movimento de cada junta são $\check{\theta}_{m_{i_{l}}}$ e $\hat{\theta}_{m_{i_{l}}}$ e a posição final do rotor é $\tilde{\theta}_{m_{i_{l}}}$. Todos estes valores devem ser fornecidos previamente.

Este tipo de problema é muito difícil de resolver por envolver um grande esforço computacional, consequência das suas não-linearidades e da grande quantidade restrições (Kirk, 1998). Normalmente as soluções são obtidas via métodos numéricos que nem sempre garantem a convergência a um mínimo global. Além disto, o cálculo das soluções dos problemas de controle ótimo devem ser rápidos em um eventual controle em tempo-real. Assim, devido à existência destas dificuldades, é que surgiu a idéia do algoritmo de controle ótimo apresentado a seguir. 


\subsubsection{Discretização}

Definamos agora um $\Delta t=\frac{t_{f}}{N}=t_{j}-t_{j-1} \forall j \in[1, N]$ onde $N$ é suficientemente grande para obter uma solução suave. Definamos também a função $u_{i_{l}}\left(t_{j}\right)$ como uma função constante por trechos, de forma tal que $u_{i_{l}}(t)=u_{i_{l}}\left(t_{j}\right), \forall t \in\left[t_{j-1}, t_{j}\right)$

Assim a solução de (5.11) fica:

$$
x_{m_{i_{l}}}\left(t_{j}\right)=e^{\bar{A}_{i_{l}} t_{j}} x_{i_{l}}(0)+e^{\bar{A}_{i_{l}} t_{j}} \Gamma_{i_{l}} I_{i_{l}}\left(t_{j}\right) U_{i_{l}}
$$

onde:

$$
\begin{gathered}
\Gamma_{i_{l}}=\bar{B}_{i_{l}}\left[\int_{0}^{t_{1}} e^{-\bar{A}_{i_{l}} t} d t+\ldots+\int_{t_{N-1}}^{t_{N}} e^{-\bar{A}_{i_{l}} t} d t\right] \\
U_{i_{l}}=\left[\begin{array}{llll}
u_{i_{l}}\left(t_{1}\right) & u_{i_{l}}\left(t_{2}\right) & \ldots & u_{i_{l}}\left(t_{N}\right),
\end{array}\right]^{T}
\end{gathered}
$$

e

$$
I_{i_{l}}\left(t_{j}\right)=\left[\begin{array}{cccc}
e_{1} & 0 & 0 & 0 \\
0 & e_{2} & 0 & 0 \\
0 & 0 & \ddots & 0 \\
0 & 0 & 0 & e_{N}
\end{array}\right]
$$

sendo

$$
e_{z}= \begin{cases}0 & , j<z \leq N \\ 1 & , 1 \leq z \leq j\end{cases}
$$

Utilizando as equações (5.24) a (5.28) o índice de desempenho pode agora ser reescrito como:

$$
\varepsilon_{i_{l}}=\min _{U_{i_{l}}} \sum_{j=1}^{N} P_{i_{l}}\left(t_{j}\right) \Delta t .
$$


Desenvolvendo o somatório de (5.29) o problema pode ser reformulado como um Problema de Programação Quadrática (PPQ) onde:

$$
\varepsilon_{i_{l}}=\min _{U_{i_{l}}} \frac{1}{2} U_{i_{l}}^{T} Q_{i_{l}} U_{i_{l}}+T_{i_{l}}^{T} H_{i_{l}} U_{i_{l}}+S_{i_{l}}
$$

sujeito às restrições:

$$
\begin{gathered}
{\left[\begin{array}{c}
\theta_{m_{i_{l}}}\left(t_{N}\right) \\
\dot{\theta}_{m_{i_{l}}}\left(t_{N}\right)
\end{array}\right]=\left[\begin{array}{c}
\tilde{\theta}_{m_{i_{l}}} \\
0
\end{array}\right]} \\
\left|i_{a_{i_{l}}}\left(t_{j}\right)\right| \leq I_{\max _{i_{l}}} \forall j=1,2, \ldots, N \\
\left|V_{a_{i_{l}}}\left(t_{j}\right)\right| \leq V_{\max _{i_{l}}} \forall j=1,2, \ldots, N \\
\check{\theta}_{m_{i_{l}}} \leq \theta_{m_{i_{l}}}\left(t_{j}\right) \leq \hat{\theta}_{m_{i_{l}}} \forall j=1,2, \ldots, N
\end{gathered}
$$

e onde os parâmetros da equação quadrática (5.30) são os seguintes:

$$
\begin{aligned}
& Q_{i_{l}}=2 \frac{R_{a_{i_{l}}}}{K_{m_{i_{l}}}^{2}} I_{i_{l}}\left(t_{N}\right), \\
& H_{i_{l}}=2 \frac{R_{a_{i_{l}}} r_{i_{l}}}{K_{m_{i_{l}}}^{2}} I_{i_{l}}\left(t_{N}\right)
\end{aligned}
$$

e

$$
S_{i_{l}}=T_{i_{l}}^{T}\left(\frac{R_{a_{i_{l}}} r_{i_{l}}^{2}}{K_{m_{i_{l}}}^{2}} I_{i_{l}}\left(t_{N}\right)\right) T_{i_{l}} .
$$

Em (5.30), as matrizes $Q_{i_{l}}, H_{i_{l}}$ e $S_{i_{l}}$ dependem somente dos parâmetros do motor e o vetor $T_{i_{l}}=\left[\tau_{i_{l}}\left(t_{1}\right), \tau_{i_{l}}\left(t_{2}\right), \ldots, \tau_{i_{l}}\left(t_{N}\right)\right]^{T}$. Logo, a solução do problema dado pelas equações (5.29) até (5.34) pode ser eficientemente encontrada a partir de métodos numéricos simples (Winston, 1995).

\subsection{Apresentação do algoritmo}

A idéia por trás do algoritmo é tentar resolver de forma iterativa o PPQ utilizando as vantagens da programação quadrática e o fato de que o controle utilizado 
é independente por junta. Espera-se que em cada interação o algoritmo convirja a um ponto de mínimo global da função objetivo (5.30).

Para esclarecer melhor a explicação a seguir, serão apresentadas as seguintes definições:

\section{Definição 1}

Dado um vetor qualquer $\mathcal{X}$ a representação $\mathcal{X}_{i_{l}}^{k}\left(t_{j}\right)$ significa que o vetor corresponde à $i$-ésima junta $\forall i=1,2, \ldots, M$ da l-ésima perna $\forall l=1,2,3,4$, no intervalo $\left[t_{j}, t_{j+1}\right) t_{j}, \forall j=1,2, \ldots, N$ e na iteração $k: k \in \mathbb{N}$.

\section{Definição 2}

Dados os vetores $T_{i_{l}}^{k} ; i=1, \ldots, M ; l=1, \ldots, L ; k=0,1,2 \ldots$, é definido o vetor $\hat{T}^{k} \in \mathbf{R}$ como:

$$
\hat{T}^{k}=\left[\begin{array}{c}
T_{1_{1}}^{k} \\
\vdots \\
T_{M_{1}}^{k} \\
T_{1_{2}}^{k} \\
\vdots \\
T_{M_{L}}^{k}
\end{array}\right]
$$

onde $\mathbf{R}$ é definido em seguida.

\section{Definição 3}

$\mathbf{R}$ é um subconjunto fechado do espaço de Banach, definido como uma bola de raio $\hat{\tau}$, em torno do ponto $\hat{\mathbf{T}} \in \mathbb{R}^{L M N}$ :

$$
\mathbf{R}(\hat{\mathbf{T}}, \hat{\tau})=\left\{\hat{T}^{k} ;\left\|\hat{T}^{k}-\hat{\mathbf{T}}\right\| \leq \hat{\tau}\right\}
$$

onde $\hat{\tau}=\max \tilde{\tau}_{m_{i_{l}}} ; i=1, \ldots, M ; l=1, \ldots, L$ e $\tilde{\tau}_{m_{i_{l}}}$ é o torque máximo do motor da junta $i_{l}$.

O algoritmo proposto pode ser representado por dois ciclos: um externo e outro interno. Para explicar melhor ao leitor o raciocínio seguido na execução do algoritmo suponha-se que a condição de partida seja dada pelo vetor $\hat{T}^{0}$. Usualmente 
o valor inicial escolhido é $T_{i_{l}}^{0}=\mathbf{0} \forall i=1, \ldots, M$ e $\forall l=1, \ldots, L$. A partir deste valor inicial são resolvidos os diferentes PPQs para o conjunto das juntas escolhidas dentro da cadeia cinemática controlada. Admita-se então que no passo $k-1$ tenha sido obtida a aproximação $T_{i_{l}}^{k-1}$. Como resultado das soluções dos PPQs será obtida uma lei de controle ótimo $\bar{U}_{i_{l}}^{k}$ que será utilizada como entrada do sistema (5.11) na geração das grandezas cinemáticas do motor $\left(\theta_{m_{i_{l}}}^{k}\left(t_{j}\right), \dot{\theta}_{m_{i_{l}}}^{k}\left(t_{j}\right)\right.$ e $\left.\ddot{\theta}_{m_{i_{l}}}^{k}\left(t_{j}\right)\right)$. Uma vez multiplicados estes valores pela relação de redução das engrenagens $r_{i_{l}}$ de cada junta são obtidas as respectivas posições, velocidades e acelerações angulares das juntas (Figura 5.3).

Com os valores de $\theta_{i_{l}}^{k}\left(t_{j}\right), \dot{\theta}_{i_{l}}^{k}\left(t_{j}\right)$ e $\ddot{\theta}_{i_{l}}^{k}\left(t_{j}\right)$ calculados é possível então obter o novo vetor de torques da cadeia cinemática por meio da solução da equação do espaço de configuração (5.16). Este novo vetor de toques $\left(\hat{T}^{k}\right)$ é logo comparado com aquele utilizado para obtê-lo $\left(\hat{T}^{k-1}\right)$ na iteração anterior (Equação (5.40)). Se o resultado da norma infinito da diferença entre estes vetores for maior que a tolerância $\xi>0$ escolhida, uma nova iteração do algoritmo será iniciada, porém, utilizando agora como ponto de partida o último vetor de torques $\left(\hat{T}^{k}\right)$ calculado (Figura 5.4).

$$
\left\|\hat{T}^{k}-\hat{T}^{k-1}\right\|_{\infty} \leq \xi
$$

Desta forma obteve-se um ciclo iterativo que deve convergir a um ponto de mínimo global como será demonstrado na seção a seguir.

\subsection{Análise da convergência do algoritmo}

A primeira condição para que o algoritmo proposto convirja é que, no PPQ das equações (5.29) a (5.34) a matriz $Q_{i_{l}}$ da equação (5.30) seja semi-definida positiva (Henrici, 1964; Giorgio et al., 2004).

$$
\nabla^{2} \varepsilon_{i_{l}}=Q_{i_{l}} \geq 0
$$




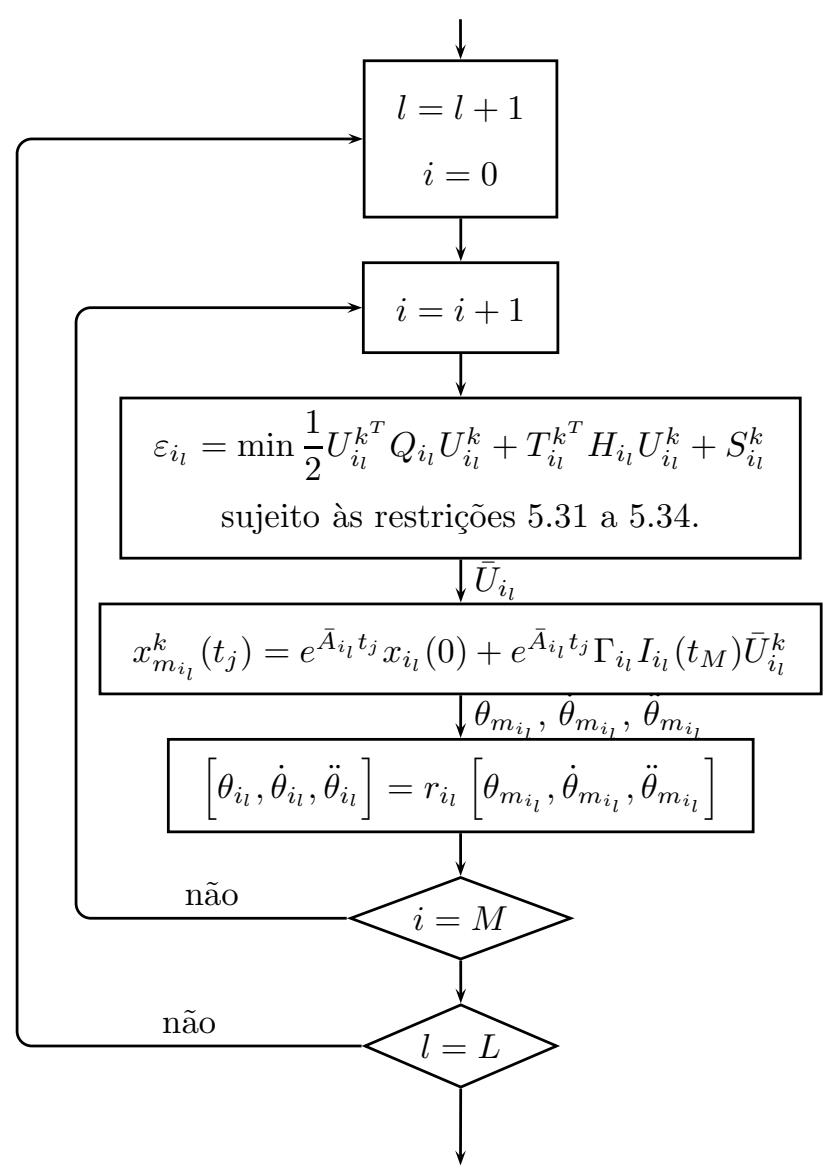

Figura 5.3: Ciclo interno do algortimo de otimização.

Note que, de acordo com (5.35), esta condição sempre será satisfeita.

Satisfeita a primeira condição, o próximo passo é verificar se as condições de Karush-Kuhn-Tucker (KKT) são também satisfeitas para o PPQ.

Definamos primeiramente:

$$
\begin{gathered}
Z_{i_{l}}=e^{A t_{N}} \Gamma I_{i_{l}}\left(t_{N}\right), \\
\tilde{\Theta}_{i_{l}}=\left[\begin{array}{c}
\theta_{i_{l}}\left(t_{N}\right) \\
0
\end{array}\right], \\
F_{i_{l}}\left(t_{j}\right)=C_{i_{l}} e^{A_{i_{l}} t_{j}} \Gamma_{i_{l}} I_{i_{l}}\left(t_{j}\right),
\end{gathered}
$$




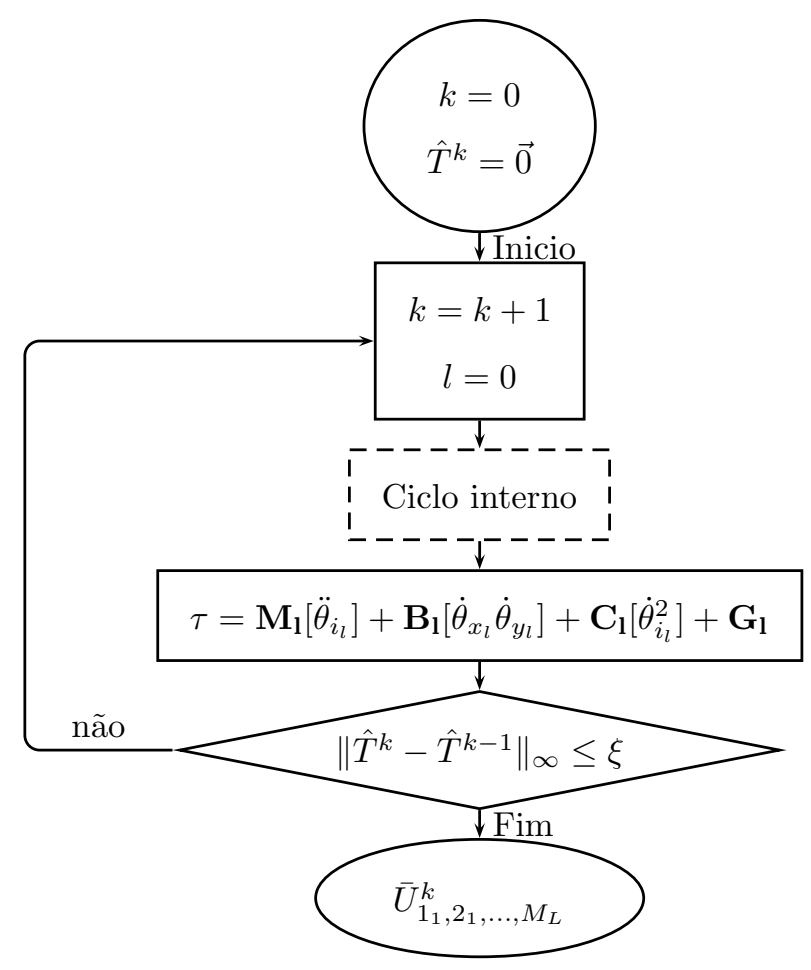

Figura 5.4: Ciclo externo do algortimo de otimização.

$$
\begin{aligned}
& G_{i_{l}}\left(t_{j}\right)= {\left[\begin{array}{ll}
0 & 1
\end{array}\right] e^{A_{i_{l}} t_{j}} \Gamma_{i_{l}} I_{i_{l}}\left(t_{j}\right), } \\
& \hat{G}_{i_{l}}=\left[\begin{array}{c}
G_{i_{l}}\left(t_{j}\right) \\
\vdots \\
G_{i_{l}}\left(t_{N}\right)
\end{array}\right]
\end{aligned}
$$

e

$$
\hat{F}_{i_{l}}=\left[\begin{array}{c}
F_{i_{l}}\left(t_{j}\right) \\
\vdots \\
F_{i_{l}}\left(t_{N}\right)
\end{array}\right] .
$$

Além disto, as funções:

$$
\begin{gathered}
g_{i_{l}}\left(U_{i_{l}}^{k}\right)=Z_{i_{l}} U_{i_{l}}^{k}-\tilde{\Theta}_{i_{l}}, \\
h_{i_{l}}\left(U_{i_{l}}^{k}\right)=U_{i_{l}}^{k}-K_{m_{i_{l}}} \hat{I}_{\text {max }_{i_{l}}}-r_{i_{l}} T_{i_{l}}^{k}, \\
p_{i_{l}}\left(U_{i_{l}}^{k}\right)=\left(\frac{R_{a_{i_{l}}}}{K_{m_{i_{l}}}} I\left(t_{N}\right)+K_{b_{i_{l}}} \hat{G}_{i_{l}}\right) U_{i_{l}}^{k}-\hat{V}_{\text {max }_{i_{l}}}-\frac{R_{a_{i_{l}}} r_{i_{l}}}{K_{m_{i_{l}}}} T_{i_{l}}^{k},
\end{gathered}
$$




$$
\check{q}_{i_{l}}\left(U_{i_{l}}^{k}\right)=\hat{F}_{i_{l}} U_{i_{l}}^{k}-\check{\Theta}_{m_{i_{l}}}
$$

e

$$
\hat{q}_{i_{l}}\left(U_{i_{l}}^{k}\right)=\hat{F}_{i_{l}} U_{i_{l}}^{k}-\hat{\Theta}_{m_{i_{l}}}
$$

onde os vetores $\hat{I}_{\max _{i_{l}}}=I_{\max _{i_{l}}}\left[1,1, \ldots, 1_{N}\right]^{T}, \hat{V}_{\max _{i_{l}}}=V_{\max _{i_{l}}}\left[1,1, \ldots, 1_{N}\right]^{T}, \check{\Theta}_{m_{i_{l}}}=$ $\left[\check{\theta}_{m_{i_{l}}}\left(t_{1}\right), \check{\theta}_{m_{i_{l}}}\left(t_{2}\right), \ldots, \check{\theta}_{m_{i_{l}}}\left(t_{N}\right)\right]^{T}$ e $\hat{\Theta}_{m_{i_{l}}}=\left[\hat{\theta}_{m_{i_{l}}}\left(t_{1}\right), \hat{\theta}_{m_{i_{l}}}\left(t_{2}\right), \ldots, \hat{\theta}_{m_{i_{l}}}\left(t_{N}\right)\right]^{T}$ representam as restrições relativas à equação (5.31) e às desigualdades (5.32), (5.33) e (5.34), respectivamente.

Logo, se os vetores $\mu_{i_{l_{r}}}, \nu_{i_{l_{r}}}, \check{\rho}_{i_{l_{r}}}, \hat{\rho}_{i_{l_{r}}} \geq 0 \quad \forall r=1,2,3, \ldots, N$ e $\lambda_{i_{l_{z}}} \geq 0 \quad \forall z=$ 1,2 existem e as seguintes condições são verificadas:

$$
\begin{gathered}
\frac{\partial \mathfrak{L}_{i_{l}}\left(U_{i_{l}}^{k}, \lambda_{i_{l}}, \mu_{i_{l}}, \nu_{i_{l}}, \check{\rho}_{i_{l}}, \hat{\rho}_{i_{l}}\right)}{\partial U_{i_{l}}^{k}}=0, \\
\frac{\partial \mathfrak{L}_{i_{l}}\left(U_{i_{l}}^{k}, \lambda_{i_{l}}, \mu_{i_{l}}, \nu_{i_{l}}, \check{\rho}_{i_{l}}, \hat{\rho}_{i_{l}}\right)}{\partial \lambda_{i_{l}}}=0,
\end{gathered}
$$

$$
\begin{gathered}
\mu_{i_{l}}^{T} h_{i_{j}}\left(U_{i_{l}}^{k}\right)=0, \\
h_{i_{l}}\left(U_{i_{l}}^{k}\right) \leq 0,
\end{gathered}
$$

$$
\nu_{i_{l}}^{T} p_{i_{j}}\left(U_{i_{l}}^{k}\right)=0
$$

$$
p_{i_{l}}\left(U_{i_{l}}^{k}\right) \leq 0
$$

$$
\check{\rho}_{i_{l}}^{T} \check{q}_{i_{j}}\left(U_{i_{l}}^{k}\right)=0
$$

$$
\check{q}_{i_{l}}\left(U_{i_{l}}^{k}\right) \leq 0
$$




$$
\hat{\rho}_{i_{l}}^{T} \hat{q}_{i_{j}}\left(U_{i_{l}}^{k}\right)=0
$$

e

$$
\hat{q}_{i_{l}}\left(U_{i_{l}}^{k}\right) \leq 0
$$

onde

$$
\begin{aligned}
\mathfrak{L}_{i_{l}}\left(U_{i_{l}}^{k}, \lambda_{i_{l}}, \mu_{i_{l}}, \nu_{i_{l}}, \check{\rho}_{i_{l}}, \hat{\rho}_{i_{l}}\right)= & \frac{1}{2} U_{i_{l}}^{k^{T}} Q_{i_{l}} U_{i_{l}}^{k}+T_{i_{l}}^{k^{T}} H_{i_{l}} U_{i_{l}}^{k}+S_{i_{l}}+\lambda_{i_{l}}^{T} g_{i_{l}}\left(U_{i_{l}}^{k}\right) \\
& +\mu_{i_{l}}^{T} h_{i_{l}}\left(U_{i_{l}}^{k}\right)+\nu_{i_{l}}^{T} p_{i_{l}}\left(U_{i_{l}}^{k}\right)+\check{\rho}_{i_{l}}^{T} \check{q}_{i_{l}}\left(U_{i_{l}}^{k}\right) \\
& +\hat{\rho}_{i_{l}}^{T} \hat{q}_{i_{l}}\left(U_{i_{l}}^{k}\right) .
\end{aligned}
$$

o PPQ tem uma única solução no ponto $\bar{U}_{i_{l}}^{k}(\mathrm{Kirk}, 1998)$.

Resolvendo as equações (5.53) até (5.62), tem-se que se as restrições não são alcançadas, o ponto de mínimo $\bar{U}_{i_{l}}$ é dado por:

$$
\bar{U}_{i_{l}}^{k}=C_{1_{i_{l}}}^{T}+C_{2_{i_{l}}} T_{i_{l}}^{k}
$$

onde:

$$
\begin{gathered}
C_{1_{i_{l}}}=\tilde{\Theta}_{i_{l}}^{T} W_{i_{l}}^{-1} Z_{i_{l}} \frac{Q_{i_{l}}^{-1}}{2} \\
C_{2_{i_{l}}}=H_{i_{l}}\left(I_{i_{l}}\left(t_{N}\right)-\frac{Q_{i_{l}}^{-1}}{2} Z_{i_{l}}^{T} W_{i_{l}}^{-1} Z_{i_{l}}\right)
\end{gathered}
$$

e

$$
W_{i_{l}}=Z_{i_{l}} \frac{Q_{i_{l}}^{-1}}{2} Z_{i_{l}}^{T}
$$

Note que $W_{i_{l}}$ e $Q_{i_{l}}$ são matrizes não-singulares.

Resolvido o PPQ resta agora demonstrar a convergência do ciclo externo do algoritmo. Para isto a solução obtida em (5.64) será utilizada para resolver (5.11). Assim as grandezas cinemáticas posição, velocidade e aceleração angular do rotor são calculadas por: 


$$
\begin{aligned}
\Theta_{m_{i_{l}}}^{k} & =\hat{F}_{i_{l}}\left[C_{1_{i_{l}}}^{T}-C_{2_{i_{l}}} T_{i_{l}}^{k}\right], \\
\dot{\Theta}_{m_{i_{l}}}^{k} & =\hat{G}_{i_{l}}\left[C_{1_{i_{l}}}^{T}-C_{2_{i_{l}}} T_{i_{l}}^{k}\right], \\
\ddot{\Theta}_{m_{i_{l}}}^{k} & =\left(\frac{1}{J_{m_{i_{l}}}}-\frac{B_{m_{i_{l}}} \hat{G}_{i_{l}}}{J_{m_{i_{l}}}}\right)\left[C_{1_{i_{l}}}^{T}-C_{2_{i_{l}}} T_{i_{l}}^{k}\right]
\end{aligned}
$$

onde $\Theta_{m_{i_{l}}}^{k}=\left[\theta_{m_{i_{l}}}^{k}\left(t_{1}\right), \theta_{m_{i_{l}}}^{k}\left(t_{2}\right), \ldots, \theta_{m_{i_{l}}}^{k}\left(t_{N}\right)\right]$. Multiplicando estas últimas equações pelo fator de redução $r_{i_{l}}$ resulta na cinemática das juntas:

$$
\left[\Theta_{i_{l}}^{k}, \dot{\Theta}_{i_{l}}^{k}, \ddot{\Theta}_{i_{l}}^{k}\right]=r_{i_{l}}\left[\Theta_{m_{i_{l}}}^{k}, \dot{\Theta}_{m_{i_{l}}}^{k}, \ddot{\Theta}_{m_{i_{l}}}^{k}\right]
$$

Os valores obtidos em (5.71) são então aplicados em (5.16). Desta forma, as expressões do trabalho virtual ou a equação do espaço de configuração podem ser reescritas como funções recursivas que dependem unicamente dos torques computados na iteração prévia $(k-1)$.

$$
\hat{T}^{k+1}=f\left(\hat{T}^{k}\right) \quad \forall k=1,2,3, \ldots
$$

onde $f: \mathbb{R}^{L M N} \rightarrow \mathbb{R}^{L M N}$.

De acordo com o Teorema dos Mapas de Contração (Henrici, 1964), se as seguintes condições são satisfeitas:

i) O conjunto de equações $f\left(\hat{T}^{k}\right)$ está definido e é contínuo na região $\mathbf{R}$.

ii) Para cada $\hat{T}^{k} \in \mathbf{R}$, existe também um $f\left(\hat{T}^{k}\right) \in \mathbf{R}$.

iii) Existe uma constante $\alpha<1$ tal que para qualquer par de pontos $\hat{T}^{x}, \hat{T}^{y} \in \mathbf{R}$ $\operatorname{com}(x, y) \in \mathbb{N}$ a seguinte inequação é satisfeita:

$$
\left\|f\left(\hat{T}^{x}\right)-f\left(\hat{T}^{y}\right)\right\| \leq \alpha\left\|\hat{T}^{x}-\hat{T}^{y}\right\|
$$

então, as consequências do teorema são: 
1. A equação iterativa 5.72 tem uma única solução $\hat{\mathcal{T}} \in \mathbf{R}$

2. Para qualquer escolha de $\hat{T}^{0} \in \mathbf{R}$ a sequência de pontos $\hat{T}^{k} \forall k=1,2,3, \ldots$ gerada por 5.72 converge a $\hat{\mathcal{T}}$.

3. Para qualquer sequência de pontos $k=1,2, \ldots$, é valida a seguinte desigualdade:

$$
\left\|\hat{T}^{k}-\hat{\mathcal{T}}\right\| \leq \frac{\alpha^{k}}{1-\alpha}\left\|\hat{T}^{2}-\hat{T}^{1}\right\|
$$

onde $\left\|\hat{T}^{k}\right\|$ é a norma euclidiana de $\hat{T}^{k}$.

A primeira condição do teorema é satisfeita uma vez que a equação (5.16) é continua e definida em R. A segunda condição é verificada sempre que na solução dos PPQs sejam respeitadas as restrições (5.31) - (5.34), já que elas garantem que o torque obtido em cada iteração pertença a $\mathbf{R}, \hat{T}^{k} \in \mathbf{R}$.

A terceira condição do teorema é chamada de condição de Lipschitz. Esta condição expressa o fato de que o mapeamento $\hat{T}^{k+1} \rightarrow f\left(\hat{T}^{k}\right)$ diminui a distância entre dois pontos quaisquer e consecutivos de $\mathbf{R}$ em pelo menos o valor do fator $\alpha$ (Hoffman, 2007). Se a função $f(\hat{T})$ tem derivadas parciais continuas na região $\mathbf{R}$, então de acordo com Henrici (1964), o limite superior de $\alpha$ pode ser escolhido como:

$$
\alpha=\max _{\hat{T} \in \mathbf{R}}\left\|\mathfrak{J}_{f}\right\|_{F}
$$

onde $\mathfrak{J}_{f}$ é a matriz Jacobiana de $f\left(\hat{T}^{k}\right)$ e a notação $\|\bullet\|_{F}$ representa a norma de Frobenius da matriz

Além disso, pode-se dizer que se o Jacobiano de (5.72) no ponto $\hat{\mathcal{T}}$ é nulo $\left(\mathfrak{J}_{f}(\hat{\mathcal{T}})=0\right)$, a convergência do algoritmo é quadrática (Henrici, 1964).

De acordo com a equação (5.16) as derivadas parciais da função $f\left(\hat{T}^{k}\right)$ existem e são continuas em R. Logo, bastaria utilizar a equação (5.75) para verificar a condição de Lipschitz e, desta forma, garantir a convergência do algoritmo. 


\section{Capítulo 6}

\section{Resultados numéricos}

\subsection{Introdução}

Neste capítulo será aplicado o algoritmo de controle ótimo proposto no capítulo precedente. Como forma de teste, o controle será feito em um ciclo completo da andadura do robô. Vale destacar que o Kamambaré, como todo robô com pernas, possui uma topologia variante no tempo que ocasiona mudanças nos graus de liberdade do sistema. A própria andadura é a responsável por estas variações já que, cada vez que uma perna se agarra à superfície de apoio ou se separa desta na procura de um novo ponto de agarramento, existem cadeias cinemáticas que fecham ou abrem.

Neste ponto do controle os modelos cinemáticos e dinâmicos da perna e da plataforma são ambos conhecidos. Logo, o controle ótimo será testado em dois diferentes modelos cinemáticos com características próprias.

\subsection{Padrão de movimentos ou andadura do robô}

As andaduras são geralmente classificadas como "simétricas"e "assimétricas", de acordo com o movimento de cada perna (Hildebrand, 1989). Esta classificação não tem nada a ver com a simetria das pernas do robô e sim em como estas se 
movimentam. Tem-se assim, que por exemplo, em uma marcha simétrica, os membros direito e esquerdo de uma diagonal se movem juntos, enquanto que em uma marcha assimétrica são os membros posteriores ou anteriores os que se movimentam ao mesmo tempo. Nestes últimos tipos de andaduras normalmente existe uma fase de suspensão na qual nenhuma perna está em contato com o solo (Figura 6.1).
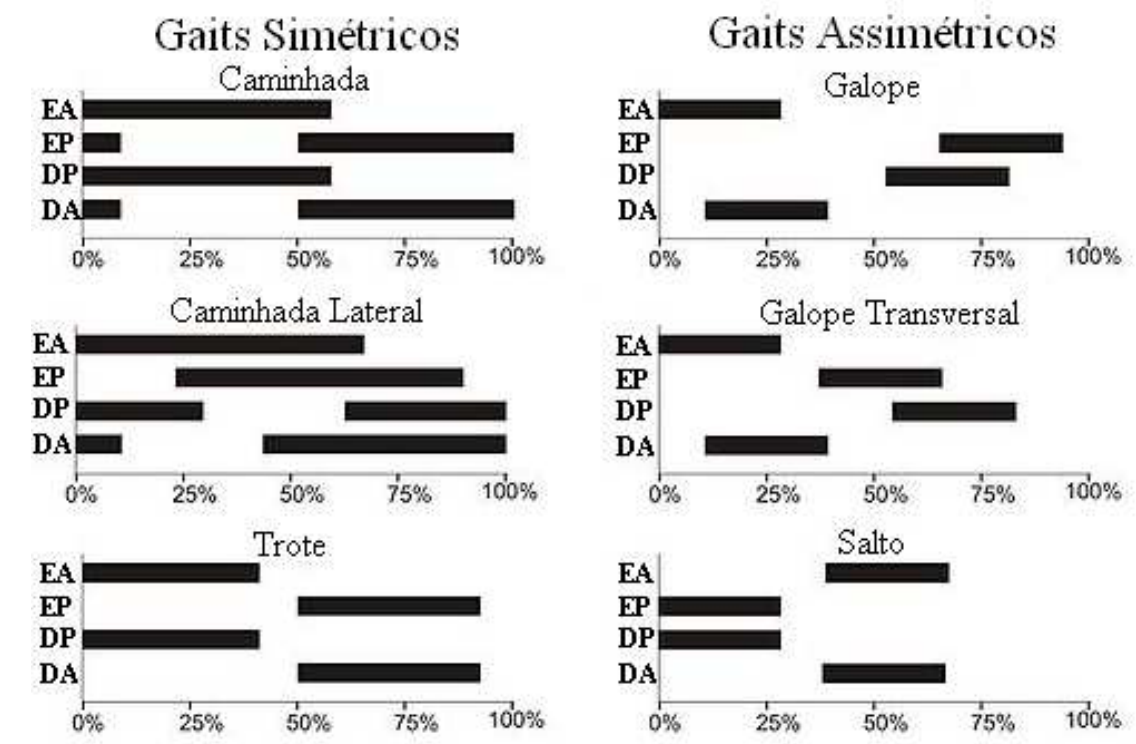

Figura 6.1: Diferentes tipos de andaduras segundo Hildebrand (1989).

Na Figura 6.1 as siglas EA, EP, DP e DA são utilizadas para denotar as pernas esquerdas e direitas, anteriores e posteriores, respectivamente. O retângulo preto é utilizado para representar o intervalo de tempo em que a perna fica em contato com a superfície de apoio.

A andadura básica do robô Kamambaré é a caminhada. Esta andadura pode ser dividida em duas fases principais: a primeira é quando a $l$-ésima perna está no ar à procura de um novo ponto de agarramento, etapas II e IV, e a segunda é quando, a partir de pelo menos 2 pontos de agarramento com a superfície, a plataforma consegue se movimentar em determinada direção ou girar em torno de seu eixo de rotação, etapas I e III.

Em cada uma das diferentes etapas da andadura, os sistemas de referência para a análise mudam. Enquanto as pernas estão no ar, a sua cinemática e dinâmica 


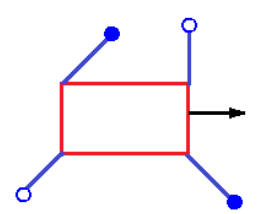

I

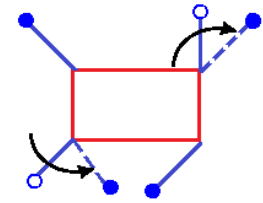

II

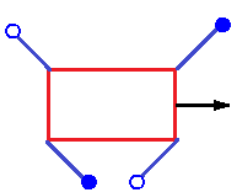

III

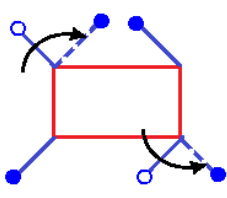

IV

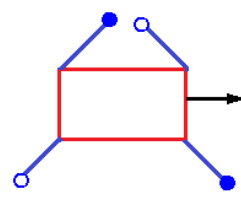

$\mathbf{V}$

Figura 6.2: Andadura do Kamambaré. o Perna no ar. • Perna de apoio.

podem ser consideradas como as de um manipulador industrial em cadeia aberta com base na plataforma e efetuador como sendo a sua garra.

Na etapa de deslocamento o robô vai se comportar como um robô paralelo devido ao fato de que existe pelo menos uma cadeia fechada que une a plataforma com a superfície de agarramento. Neste caso a análise cinemática é mais complicada, pois as cadeias fechadas introduzem restrições no movimento, e eventualmente, variações nos graus de liberdade do robô (Merlet, 2006).

\subsection{Classificação das juntas}

De acordo com o mencionado em seções anteriores, todos os motores que controlam as juntas do robô são de C.C., e neste caso particular, iguais, porém suas funções são distintas. Temos assim, que no robô podem existir juntas passivas e juntas ativas. O conjunto das juntas passivas será denotado por $\mathcal{P}$ enquanto o conjunto das juntas ativas será representado por $\mathcal{A}$. Uma junta é dita ativa quando existe uma lei de controle efetiva que atua sobre o motor que a controla, na ausência desta lei de controle, a junta é dita passiva.

Além dos conjuntos definidos, existem mais dois tipos de juntas no robô: as dependentes e as independentes. Esta nova classificação é produto das restrições geométricas impostas ao mecanismo. Por exemplo, discutamos o caso do movimento da plataforma enquanto as pernas 1 e 3 se encontram fixas à superfície (Figura 6.3). 


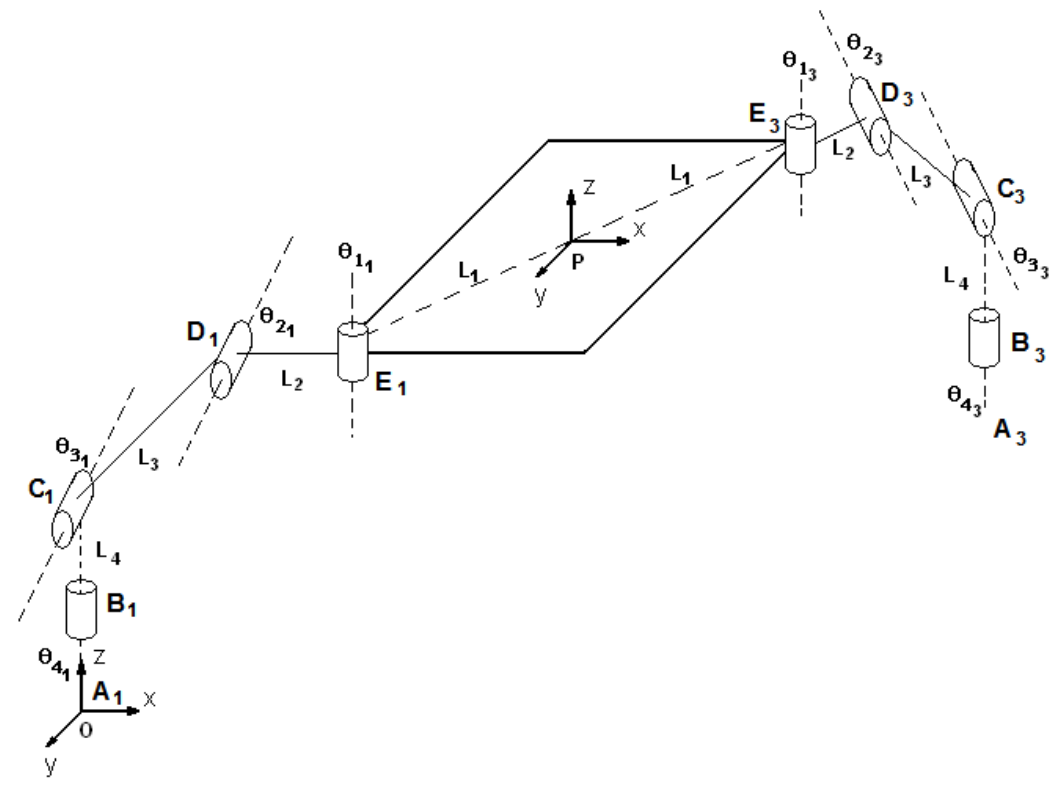

Figura 6.3: Modelo mecânico

Neste caso é necessário resolver o sistema de equações (6.1):

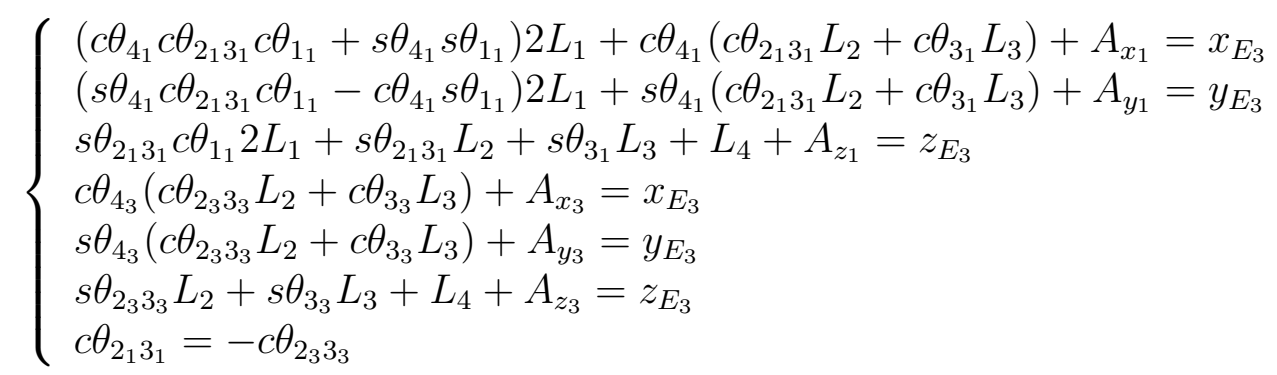

Em (6.1) as primeiras seis equações correspondem às equações de posição do ponto ${ }^{O} E_{3}$ a partir dos pontos ${ }^{O} A_{1}$ e ${ }^{O} A_{3}$ respectivamente. O sistema de coordenadas $\{O\}$ foi escolhido de tal forma que sua origem e orientação coincidem com as do sistema $\left\{{ }^{O} A_{1}\right\}$, com origem fixo ao ponto de agarramento ${ }^{O} A_{1}$, (Figura 6.3). A sétima equação é consequência da restrição de projeto do robô que impõe que os pontos ${ }^{O} D_{1},{ }^{O} E_{1},{ }^{O} P,{ }^{O} D_{3},{ }^{O} E_{3}$ estejam sempre no mesmo plano.

Voltando ao sistema (6.1), tem-se que existem 4 equações a resolver e 7 incógnitas. Logo, bastará escolher somente 3 variáveis independentes para que o valor das outras 4 possa ficar totalmente definido. O conjunto formado pelas 3 variáveis independentes será representado por $\mathcal{I}$ e tem que satisfazer a condição: $\mathcal{I} \subseteq \mathcal{A}$, já o conjunto das variáveis dependentes é denotado por $\mathcal{D}$. Dentro do con- 
junto de soluções de (6.1) sempre é escolhido aquele subconjunto de solução que esteja mais próximo ao estado anterior. A solução detalhada do sistema (6.1) pode ser estudada no Apêndice C.

Pode-se agora escolher a pertinência de cada junta aos conjuntos antes definidos. Note que os conjuntos dados na Tabela 6.1 não têm que ser necessariamente

Tabela 6.1: Classificações das juntas na andadura do Kamambaré.

\begin{tabular}{ccc}
\hline Conjuntos & $\begin{array}{c}\text { Movimento da plataforma } \\
\text { Pernas de apoio } l=1,3\end{array}$ & $\begin{array}{c}\text { Movimento das pernas no ar } \\
\text { Pernas } l=2,4\end{array}$ \\
\hline $\mathcal{A}$ & $\left\{\theta_{2_{1}}, \theta_{3_{1}}, \theta_{4_{1}}, \theta_{2_{3}}, \theta_{3_{3}}, \theta_{4_{3}}\right\}$ & $\left\{\theta_{1_{2}}, \theta_{2_{2}}, \theta_{3_{2}}, \theta_{1_{4}}, \theta_{2_{4}}, \theta_{3_{4}}\right\}$ \\
$\mathcal{P}$ & $\left\{\theta_{1_{1}}, \theta_{1_{3}}\right\}$ & $\left\{\theta_{4_{2}}, \theta_{4_{4}}\right\}$ \\
$\mathcal{I}$ & $\left\{\theta_{2_{3}}, \theta_{3_{3}}, \theta_{4_{3}}\right\}$ & $\left\{\theta_{1_{2}}, \theta_{2_{2}}, \theta_{3_{2}}, \theta_{1_{4}}, \theta_{2_{4}}, \theta_{3_{4}}\right\}$ \\
$\mathcal{D}$ & $\left\{\theta_{2_{1}}, \theta_{3_{1}}, \theta_{4_{1}}, \theta_{1_{1}}, \theta_{1_{3}}\right\}$ & $\{\emptyset\}$ \\
\hline
\end{tabular}

únicos. Um outro ponto de interesse é que, no controle, só se levam em consideração os motores das juntas e não os que controlam a operação da garra. Neste sentido um outro problema de controle pode ser fruto de futuras pesquisas.

\subsection{Condições prévias}

Antes de aplicar o algoritmo proposto no capítulo anterior é necessário comprovar se a condição de Lipschitz, é satisfeita ou não. Para isto as equações de torque da plataforma (4.13) ou da perna (4.25) são colocadas na forma da equação de espaço de configuração. Seguidamente suas derivadas parciais são calculadas e então por médio da equação (5.75) é verificada a condição de Lipschitz. Uma vez que dita condição é satisfeita para o deslocamento das pernas e da plataforma, é possível utilizar o algoritmo de optimização para o controle do robô Kamambaré.

Feitas as verificações anteriores, é possível agora projetar um esquema de controle para a andadura do robô, mas, antes de prosseguir, é necessário apresentar 
algumas condições que foram assumidas nos testes realizados:

1. $\mathrm{O}$ vetor ${ }^{O} \overrightarrow{A_{l} B_{l}}$ é sempre ortogonal à superfície de apoio.

2. Enquanto a plataforma está deslocando-se, as pernas que se encontram no ar estão travadas.

3. A andadura tem inicio com o deslocamento da plataforma.

4. Não foi considerada a presença de obstáculos no deslocamento do robô.

Outros dados de interesse para o controle são apresentados na Tabela 6.2.

Tabela 6.2: Grandezas físicas do robô

\begin{tabular}{cccc}
\hline Ligamento & Comprimento $(\mathrm{m})$ & Peso $(\mathrm{kg})$ & Momento Inércia $\left(\mathrm{kgm}^{2}\right)$ \\
\hline $5\left(\overrightarrow{A_{l} B_{l}}\right)$ & 0.2 & 0.25 & $1.25 \cdot 10^{-5}$ \\
$4\left(\overrightarrow{B_{l} C_{l}}\right)$ & 0.2 & 0.2 & $17.6 \cdot 10^{-5}$ \\
$3\left(\overrightarrow{C_{l} D_{l}}\right)$ & 0.3 & 0.25 & $130.83 \cdot 10^{-5}$ \\
$2\left(\overrightarrow{D_{l} E_{l}}\right)$ & 0.1 & 0.06 & $1.625 \cdot 10^{-5}$ \\
Plataforma & $0.42 \times 0.42$ & 5.25 & $2932.45 \cdot 10^{-5}$ \\
\hline
\end{tabular}

No controle das juntas foi utilizado sempre o mesmo motor C.C. modelo RS540 do fabricante Mabuchi. O motor está acoplado a um mecanismo de engrenagem modelo 70103 do fabricante Tamiya. As características técnicas detalhadas de ambos os componentes podem ser encontradas nos Apêndices D e E, respectivamente. Outro dado de interesse é a corrente total consumida pelos motores das juntas ativas em cada etapa da andadura. Para fornecer a potência desejada foi escolhida uma bateria selada de 12V e 14AH modelo BT-12M14AC, detalhada no Apêndice F.

Com estas condições definidas, o esquema de controle implementado é o mostrado na Figura 6.4. Note que, antes de executar o algoritmo para o cálculo do controle ótimo, são comprovadas as restrições geométricas do sistema, através de uma verificação entre os valores desejados e sua pertinência ao espaço de trabalho do mecanismo. 


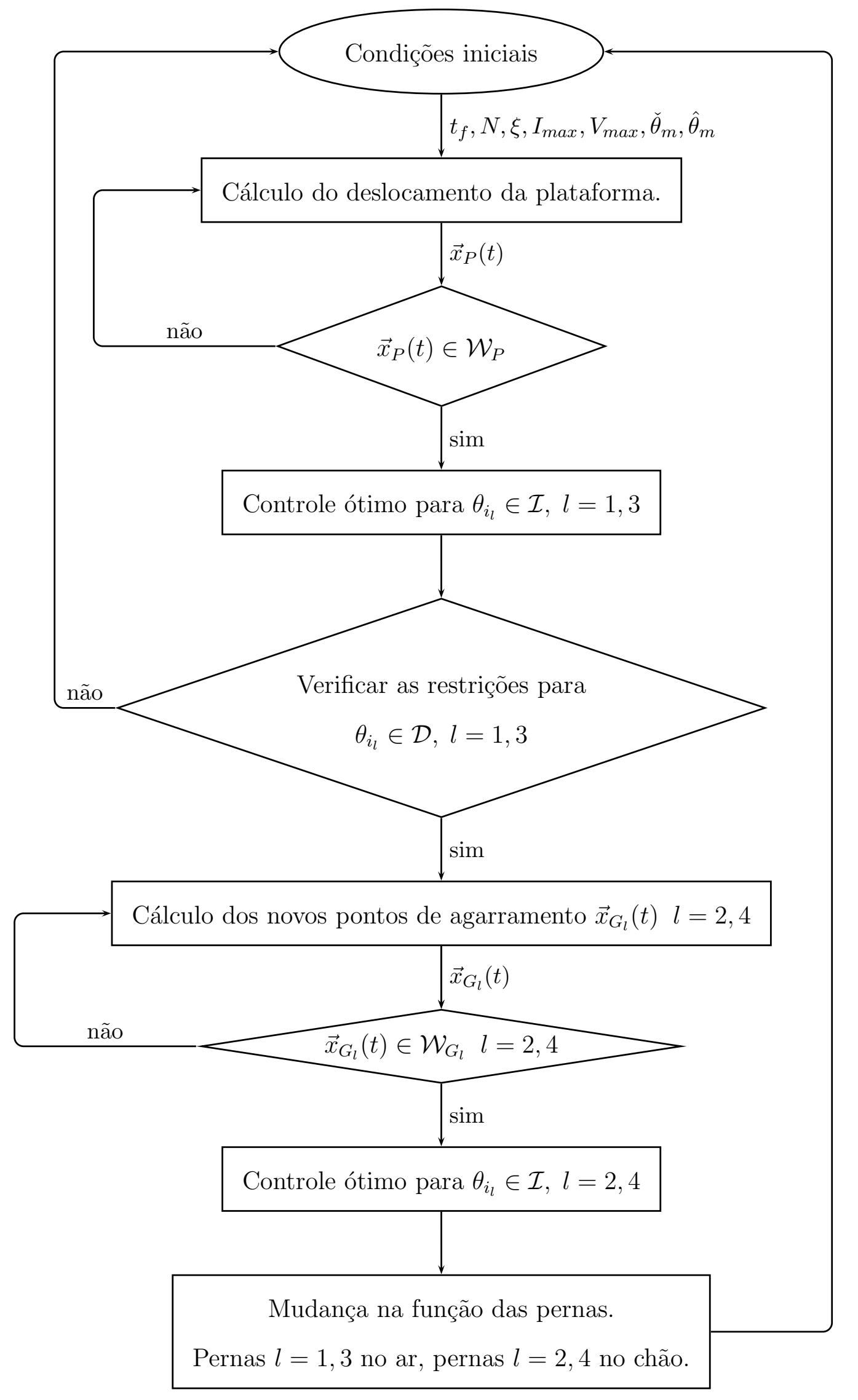

Figura 6.4: Fluxograma de controle para um ciclo básico da andadura. 


\subsection{Resultados Finais}

Nesta seção serão apresentados os resultados numéricos obtidos mediante simulações feitas no modelo do robô Kamambaré. Como foi mostrado na Figura 6.2 e no fluxograma da Figura 6.4, a andadura do robô, tem inicio com um deslocamento da plataforma (etapa I), nesta etapa as pernas $l=2,4$ estão no ar.

No exemplo a seguir será mostrado o desempenho do robô nas etapas I e II. Para isto, deseja-se primeiramente um deslocamento do robô do ponto ${ }^{O} P$ em relação ao sistema $\{O\}$ ao longo do eixo $Y$ desde uma posição inicial ${ }^{O} P(0)=[0.392,0,0.231]$ até uma posição final ${ }^{O} P(I)=[0.392,0.39,0.231]$. Assume-se também que o sistema de referência $\{O\}$ foi escolhido de forma tal que coincide com o sistema $\left\{A_{1}\right\}$ inicial. Em seguida, o movimento continua até alcançar a posição final ${ }^{O} P(I V)=$ $[0.45,0.68,2.31]$.

Na Tabela 6.3 são mostrados os parâmetros de controle utilizados para o movimento da plataforma e o movimento das pernas nas etapas I e II respectivamente.

Tabela 6.3: Parâmetros de controle

\begin{tabular}{ccc}
\hline Parâmetro & Movimento Plataforma & Movimento Pernas no ar \\
\hline$N$ & 100 & 100 \\
$t_{f}$ & $40 s$ & $60 s$ \\
$\xi$ & $10^{-7}$ & $10^{-7}$ \\
$I_{\max _{i_{l}}}$ & $3.5 \mathrm{~A}$ & $2.8 \mathrm{~A}$ \\
$V_{\max _{i_{l}}}$ & $12 \mathrm{~V}$ & $12 \mathrm{~V}$ \\
{$\left[\check{\theta}_{2_{3}} ; \hat{\theta}_{2_{3}}\right]$} & {$[0.85 ; 0.92]$} & \\
{$\left[\check{\theta}_{3_{3}} ; \hat{\theta}_{3_{3}}\right]$} & {$[2.24 ; 2.3]$} & \\
{$\left[\check{\theta}_{4_{3}} ; \hat{\theta}_{4_{3}}\right]$} & {$[-0.54 ; 0.55]$} & {$[-0.78 ; 0.59]$} \\
{$\left[\check{\theta}_{1_{2}} ; \hat{\theta}_{1_{2}}\right]$} & & {$[-0.87,0.78]$} \\
{$\left[\check{\theta}_{2_{2}} ; \hat{\theta}_{2_{2}}\right]$} & & \\
{$\left[\check{\theta}_{3_{2}} ; \hat{\theta}_{3_{2}}\right]$} & & \\
\hline
\end{tabular}


As Figuras 6.5 - 6.13 mostram as características principais do movimento da plataforma na etapa I.

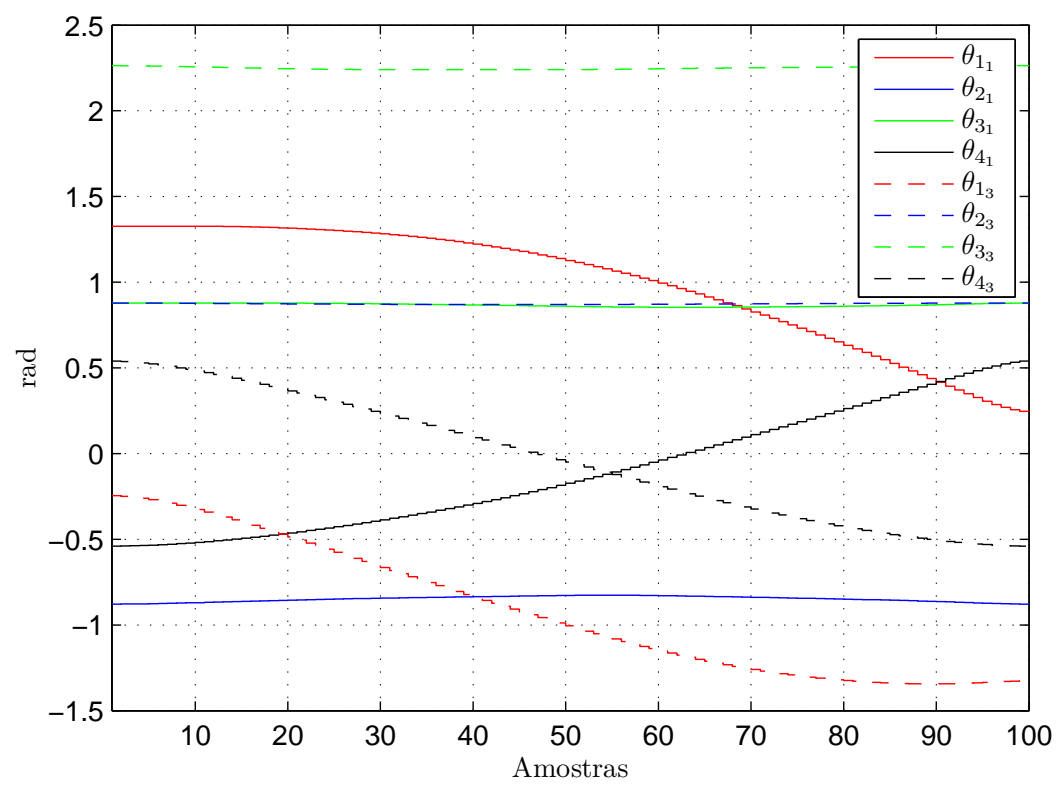

Figura 6.5: Posição angular de cada junta durante a etapa I
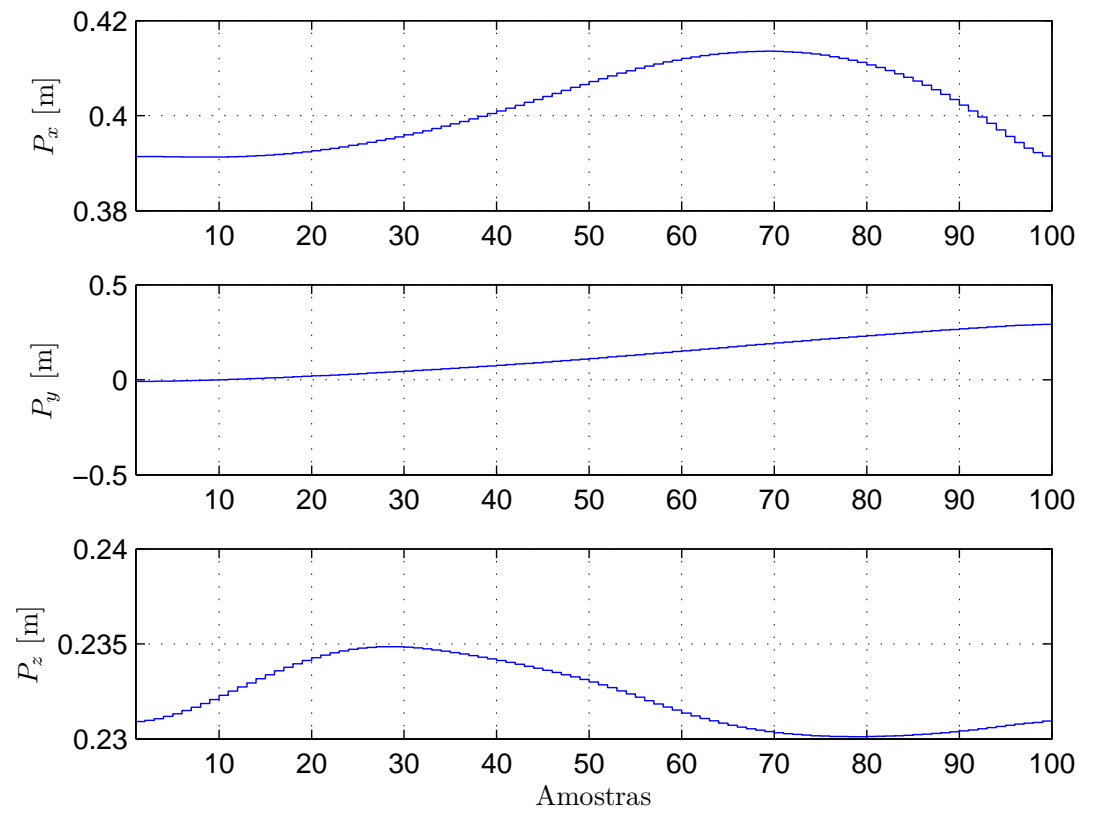

Figura 6.6: Deslocamento do centro da plataforma durante a etapa I

Após o movimento da plataforma (etapa II) as pernas que estão no ar, $l=1,3$ 

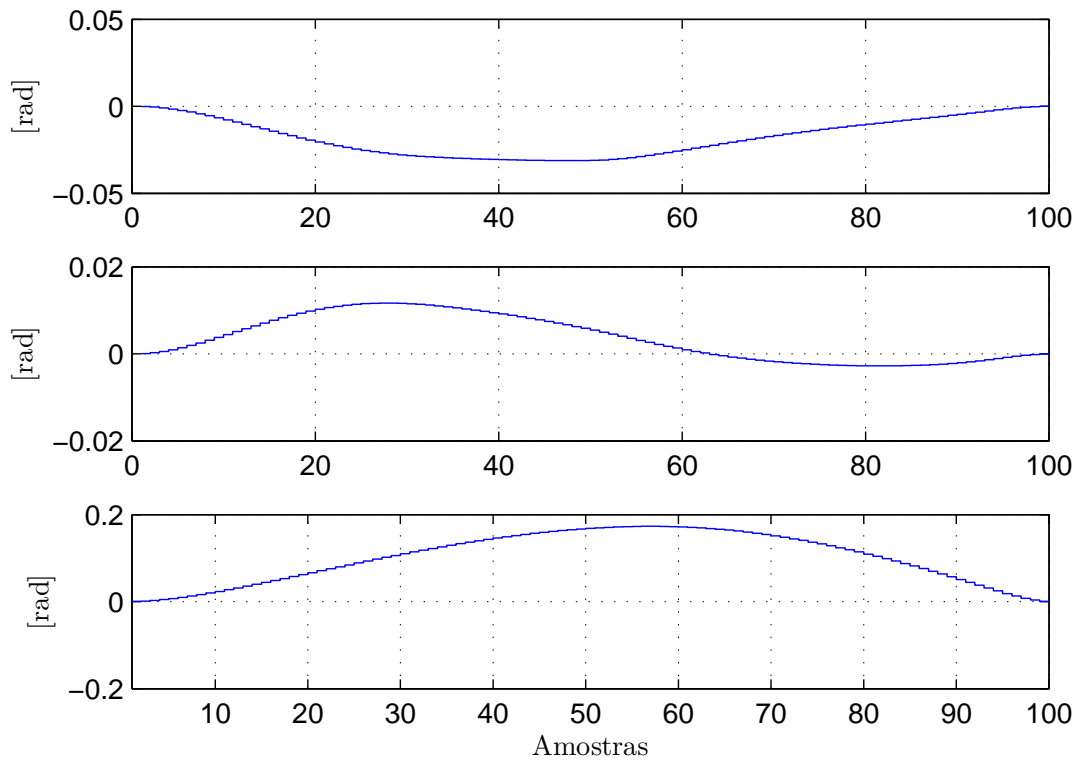

Figura 6.7: Rotações em tornos dos eixos do sistema $\{P\}$ durante a etapa I

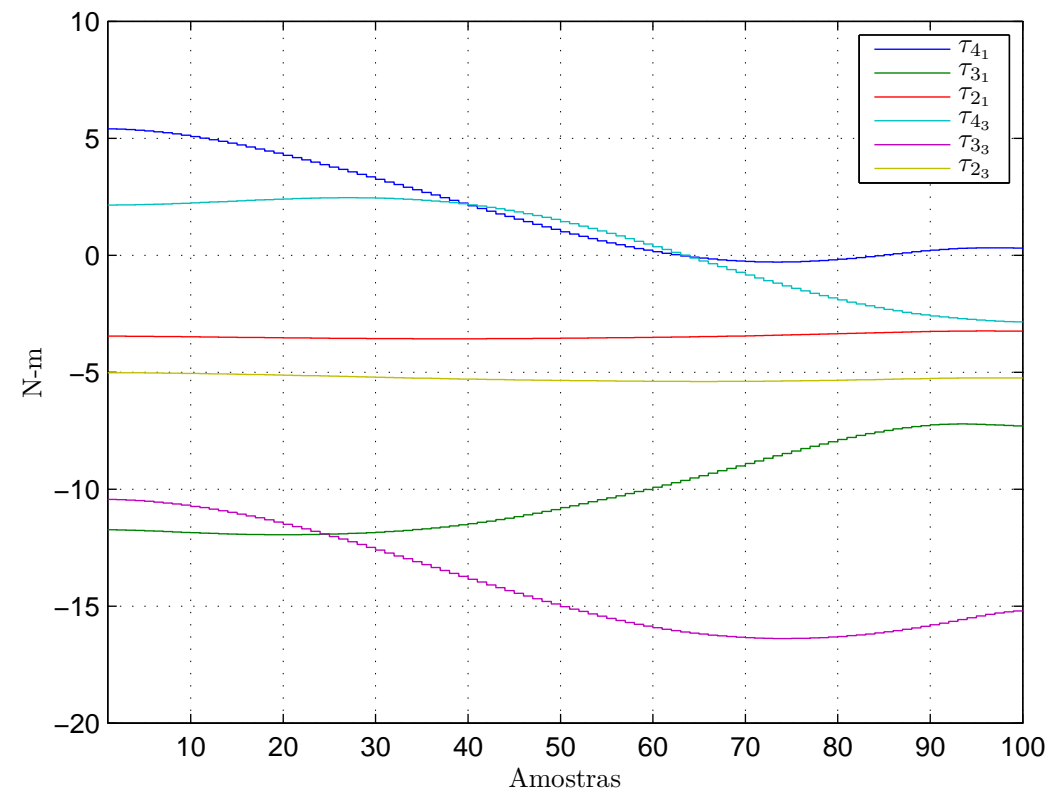

Figura 6.8: Torques de carga de cada junta durante a etapa I

são situadas nas novas posições de agarramento ${ }^{O} A_{2}(I I)$ e ${ }^{O} A_{4}(I I)$. Assim, as Figuras 6.14 - 6.21 apresentam o desempenho da perna $l=2$ no ar (etapa II).

No caso do deslocamento da plataforma, as leis de controle ótimo somente são 


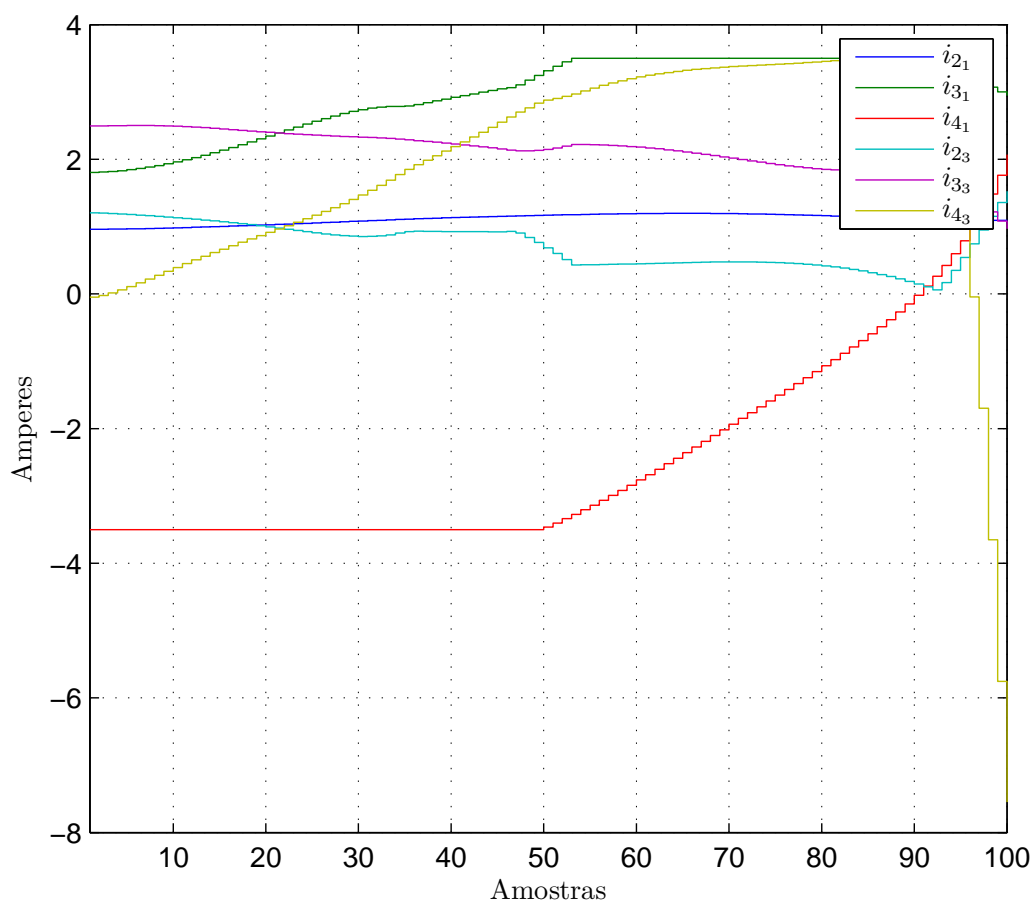

Figura 6.9: Corrente de armadura de cada junta durante a etapa I

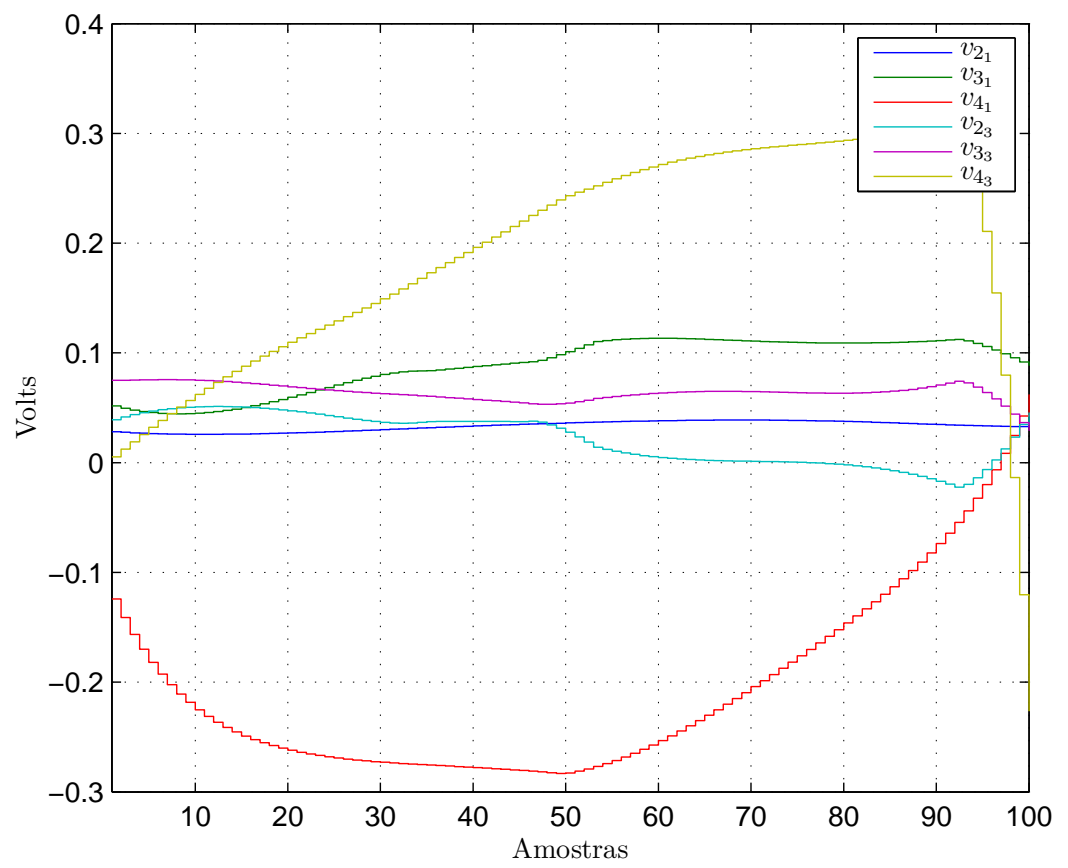

Figura 6.10: Tensão de armadura de cada junta durante a etapa I 


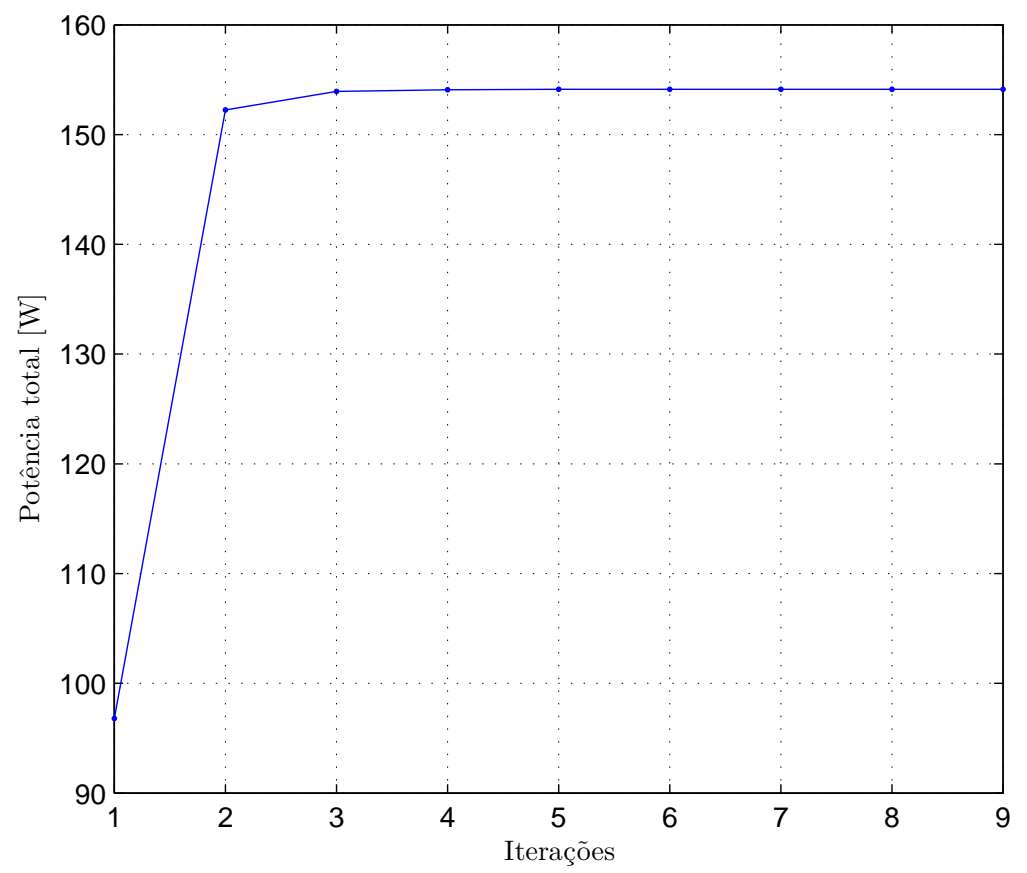

Figura 6.11: Potência total consumida pelos motores na etapa I.

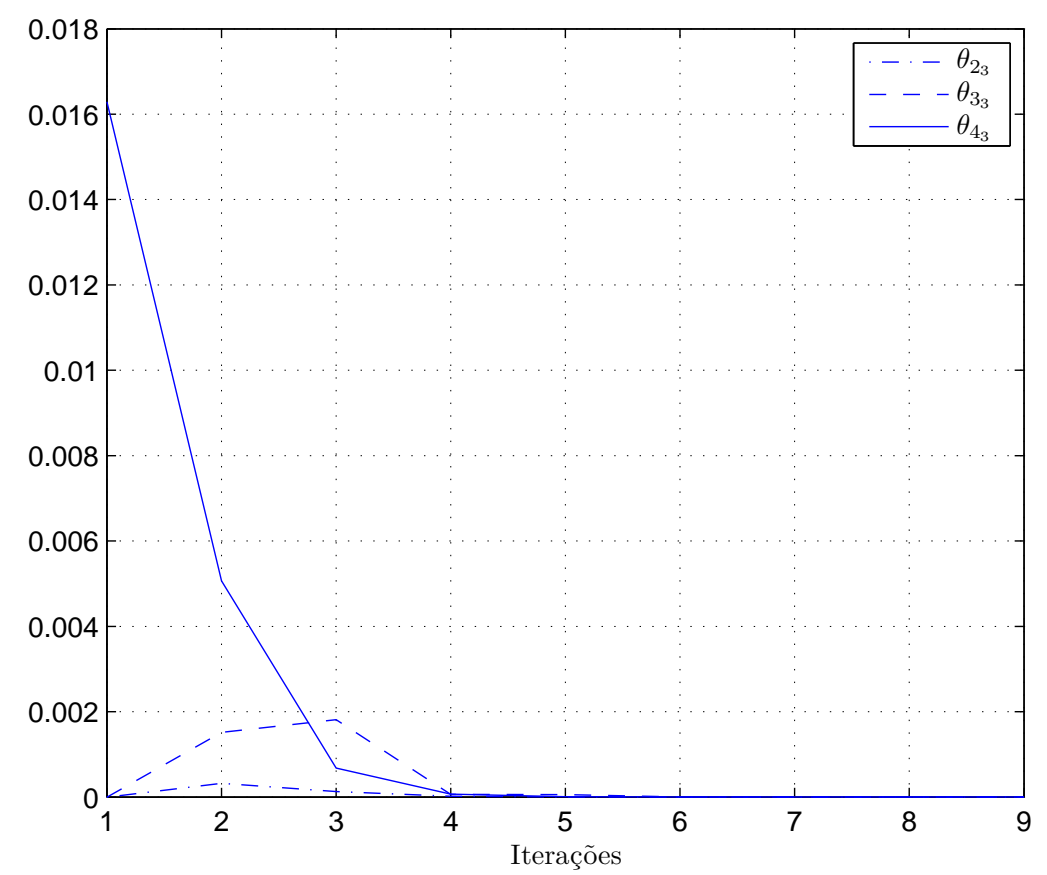

Figura 6.12: Erro entre as soluções de duas iterações consecutivas para o conjunto das juntas $\mathcal{A}$ na etapa I 


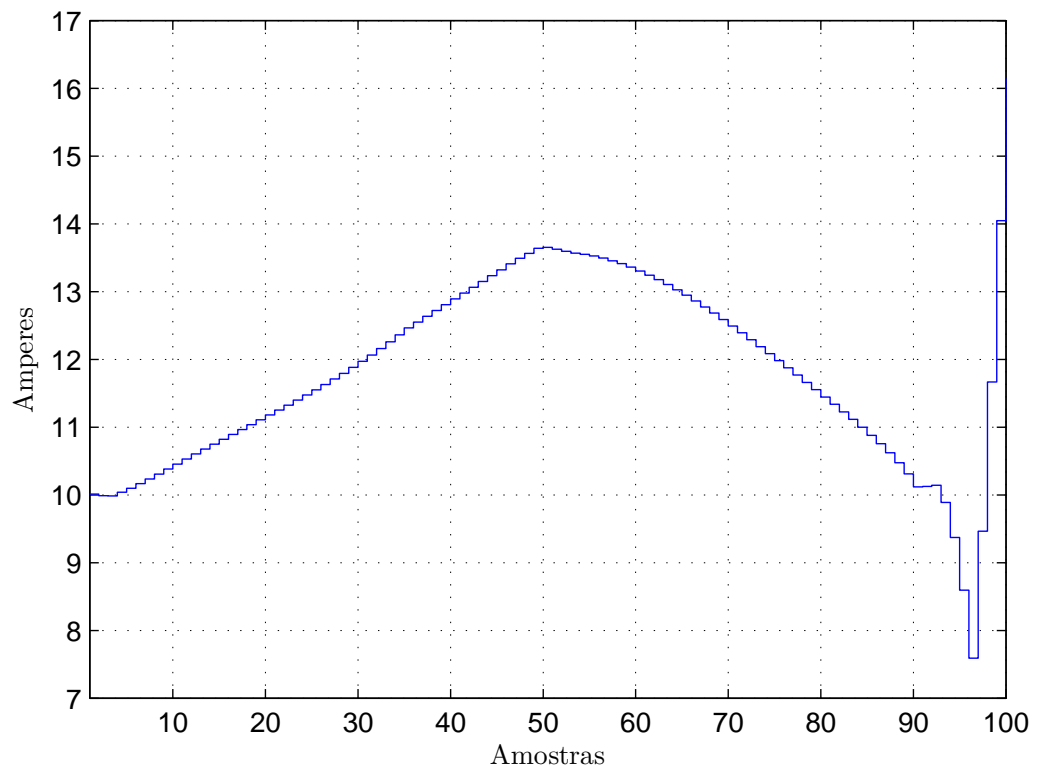

Figura 6.13: Corrente total consumida pelos motores das juntas $\mathcal{A}$ na etapa I

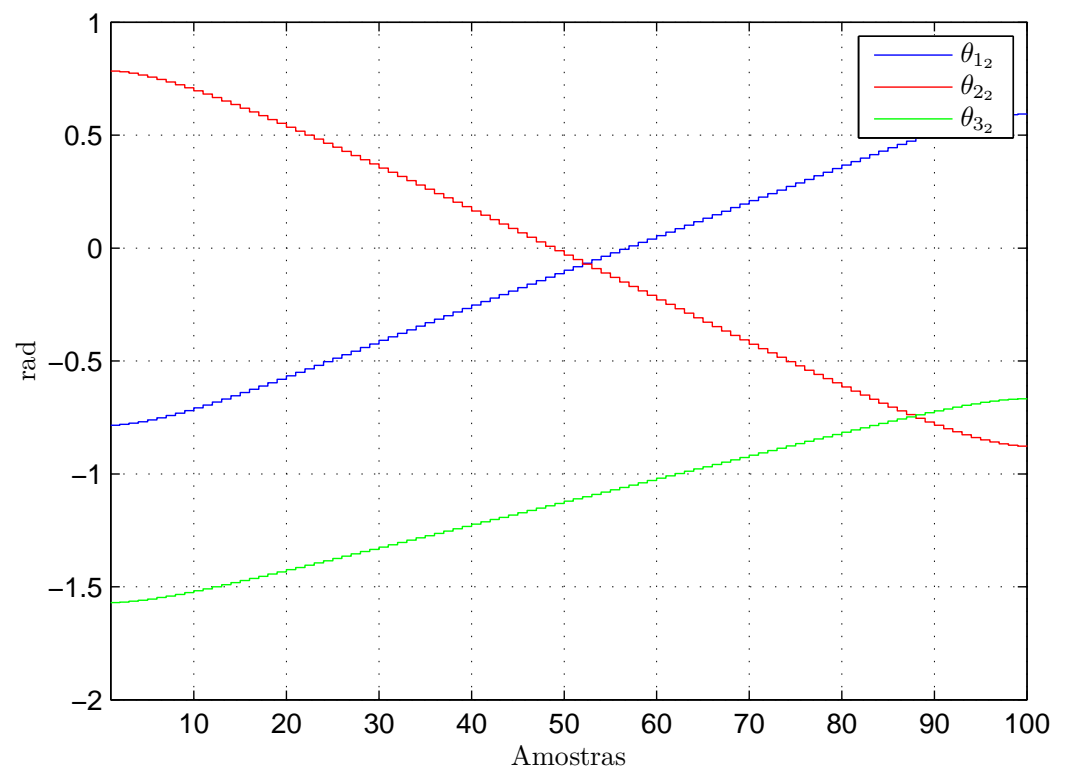

Figura 6.14: Posição angular de cada junta durante a etapa II

aplicadas ao conjunto das juntas independentes, $\mathcal{I}=\left\{\theta_{2_{3}}, \theta_{3_{3}}, \theta_{4_{3}}\right\}$. Logo, se após o cálculo da trajetória para $\mathcal{I}$, alguma junta do conjunto $\mathcal{D}$ ultrapassa as restrições elétricas do motor, procede-se ao aumento do $t_{f}$ com a finalidade de diminuir as exigências dos motores e assim mantê-los nas condições de trabalho permissíveis, 

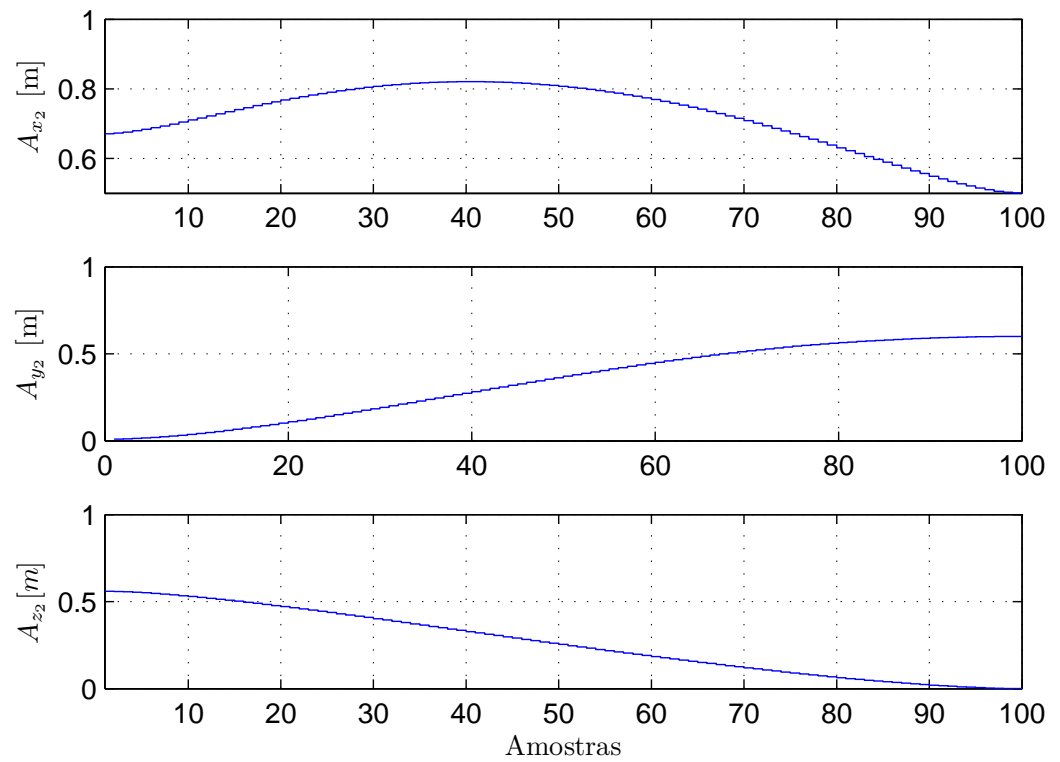

Figura 6.15: Coordenadas do ponto $A_{2}$ durante a etapa II

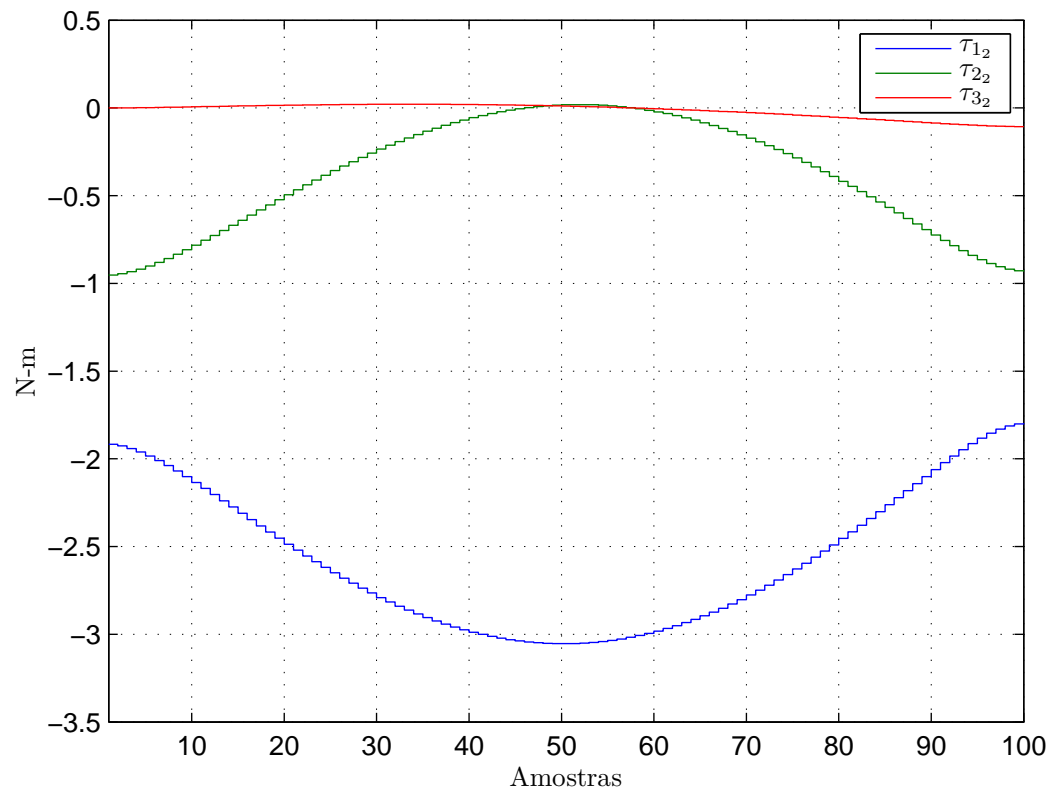

Figura 6.16: Torques de carga de cada junta durante a etapa II

Figuras 6.22 e 6.23. Este mecanismo pouco comum de controle garante que sejam respeitadas as restrições de consumo para cada junta do conjunto $\mathcal{D}$ e que possam ser mantidas as restrições lineares na hora de resolver os PPQs relativos às juntas do conjunto $\mathcal{I}$. 


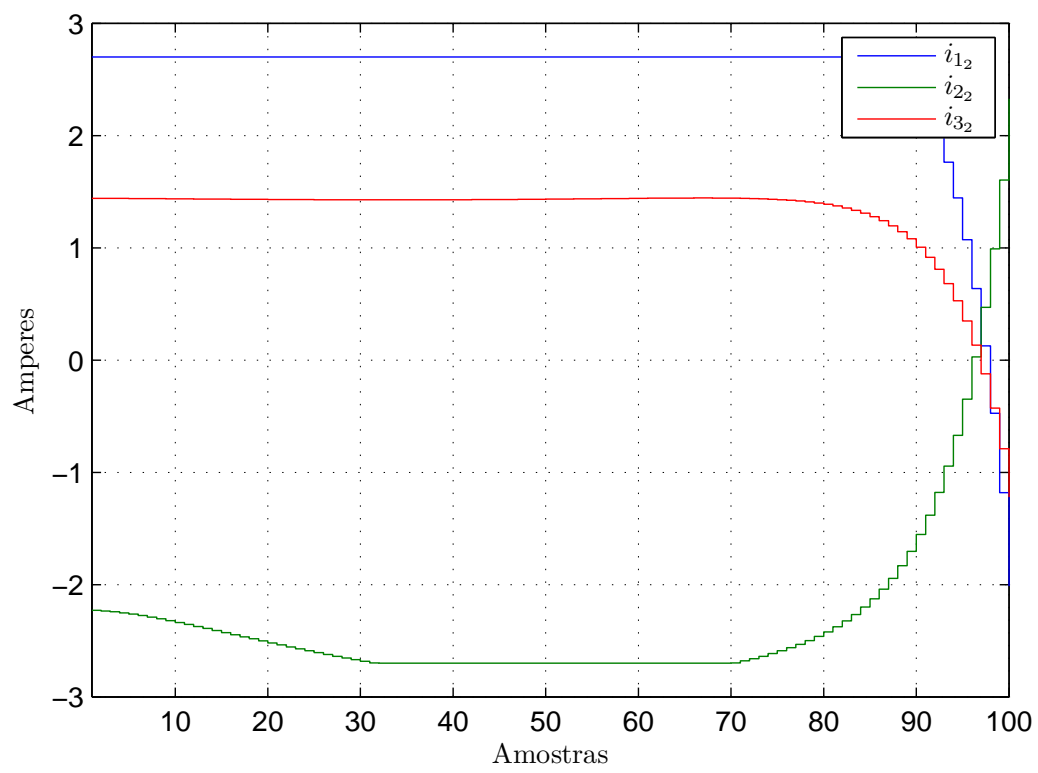

Figura 6.17: Corrente de armadura de cada junta durante a etapa II

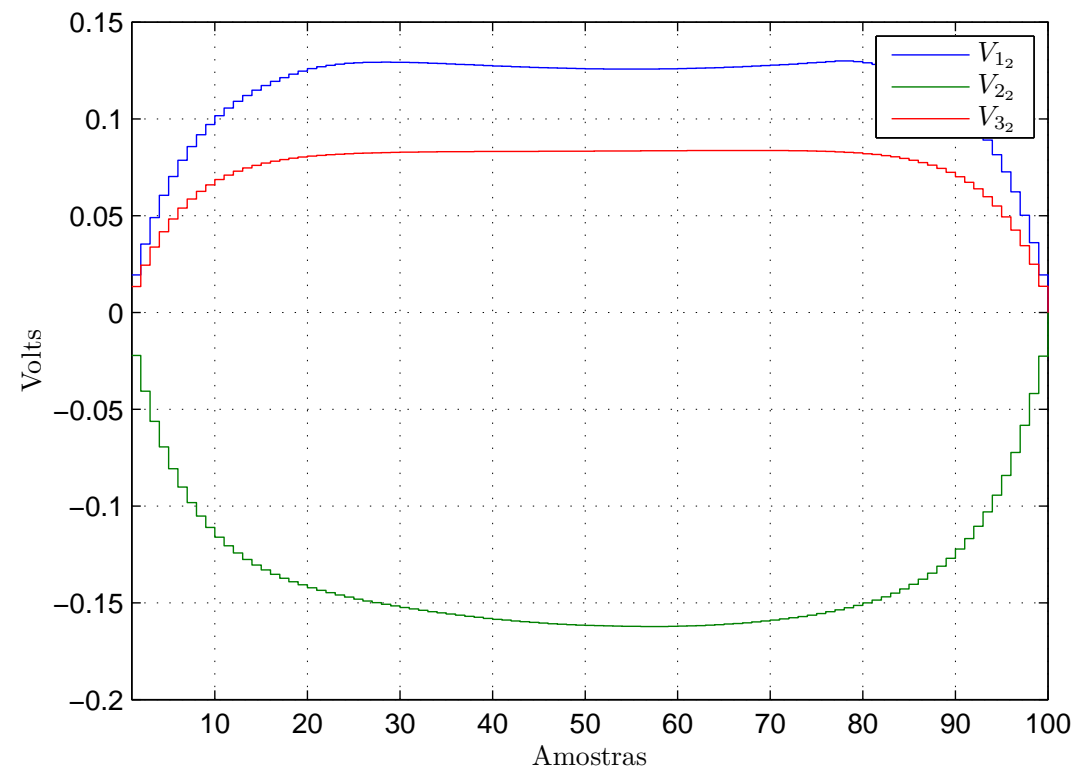

Figura 6.18: Tensão de armadura de cada junta durante a etapa II

Além da corrente consumida por cada motor, outro critério de controle utilizado foi a corrente total consumida pelo sistema em cada etapa da andadura. Para controlar este consumo, foram alterados os limites de corrente nos motores e o tempo de execução do movimento de tal forma que o consumo total não ultrapassasse de- 


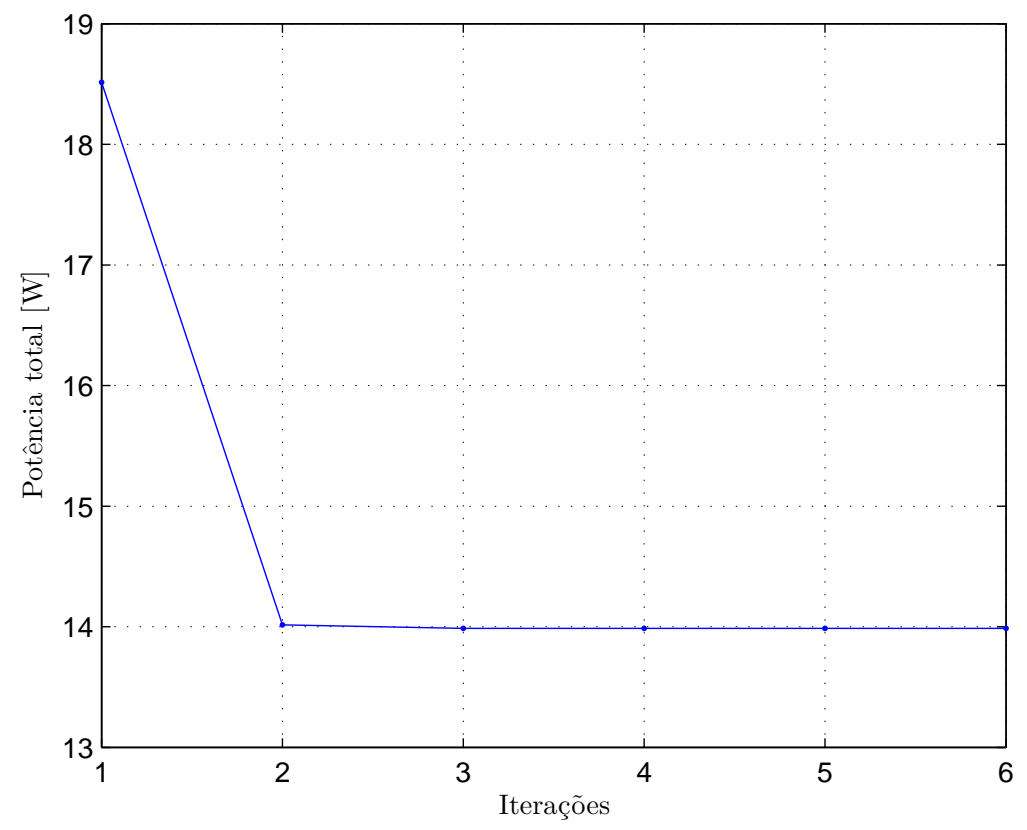

Figura 6.19: Potência total consumida pelos motores na etapa II.

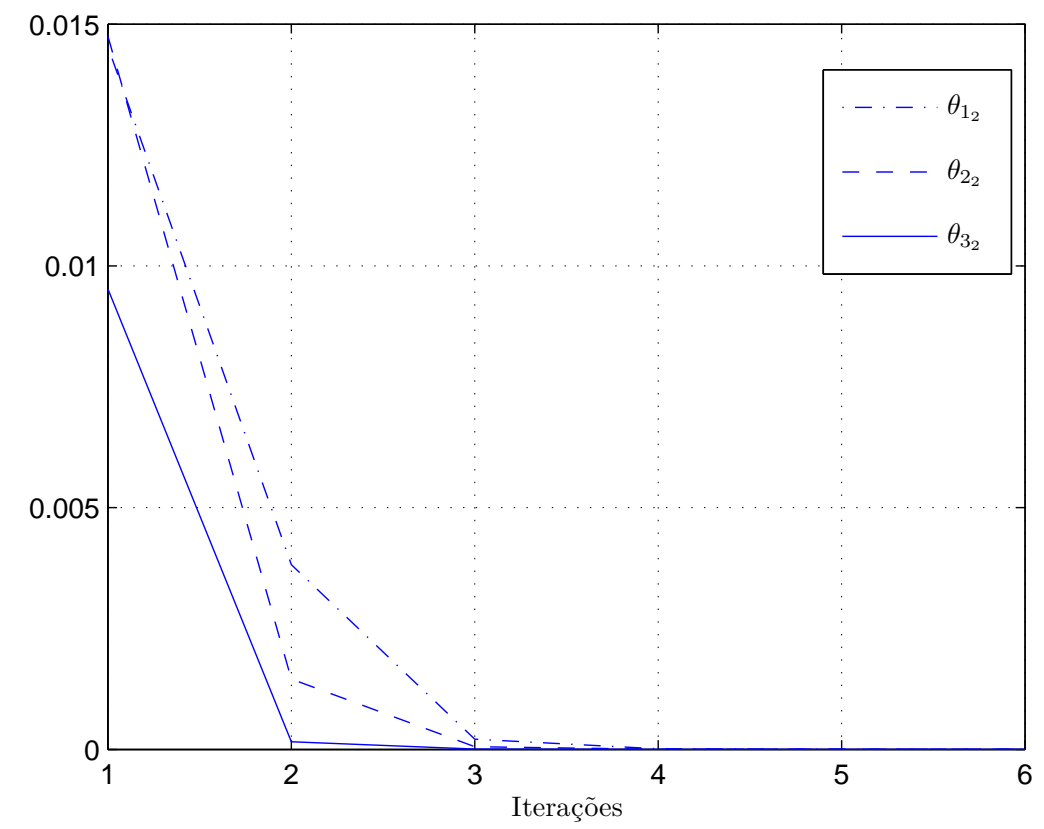

Figura 6.20: Erro entre as soluções de duas iterações consecutivas para o conjunto das juntas $\mathcal{A}$ na etapa II 


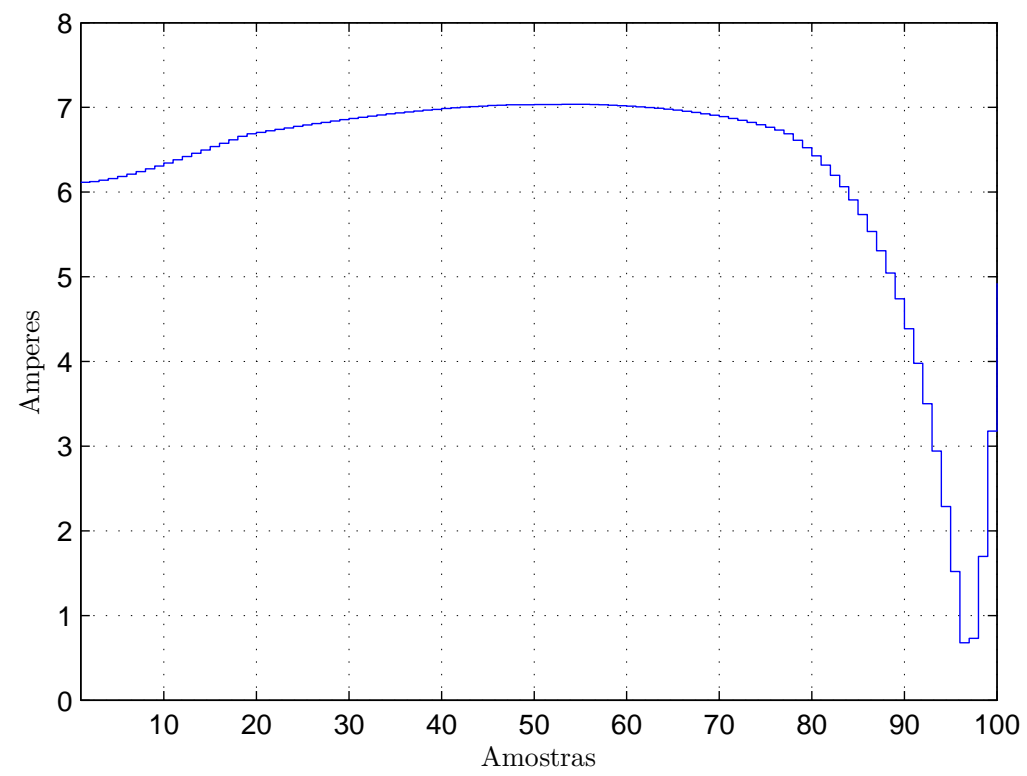

Figura 6.21: Corrente total consumida pelos motores das juntas $\mathcal{A}$ na etapa II

terminado valor. Como exemplo foi escolhido um valor máximo de 14A para cada uma das etapa de deslocamento, Figura 6.24. Note que, nas etapas em que as duas pernas estão movimentando-se no ar, o valor máximo de corrente consumido por cada perna foi limitado a 7A.

Um ponto de interesse é que de acordo as Figuras 6.11 e 6.19 o caminho até o ponto de mínimo global não é necessariamente monótono.

Finalmente na Tabela 6.4 são mostradas as iterações necessárias para a convergência do algoritmo, o tempo de processamento gasto na execução do mesmo e a potência consumida pelo robô em cada etapa da andadura. Observe que a etapa III está composta primeiramente pela elevação das pernas $l=1,3$, seguida pelo deslocamento da plataforma. No Apêndice G é apresentada a sequência completa de movimentos do robô sob a ação do algoritmo de controle para um ciclo da andadura.

Todos os testes foram realizados na plataforma MATLAB (R2008b) com sistema operacional Windows 7 de 64 bits e processador Intel(R) CORE(TM) Duo CPU T6400 a 2.00GHz. 


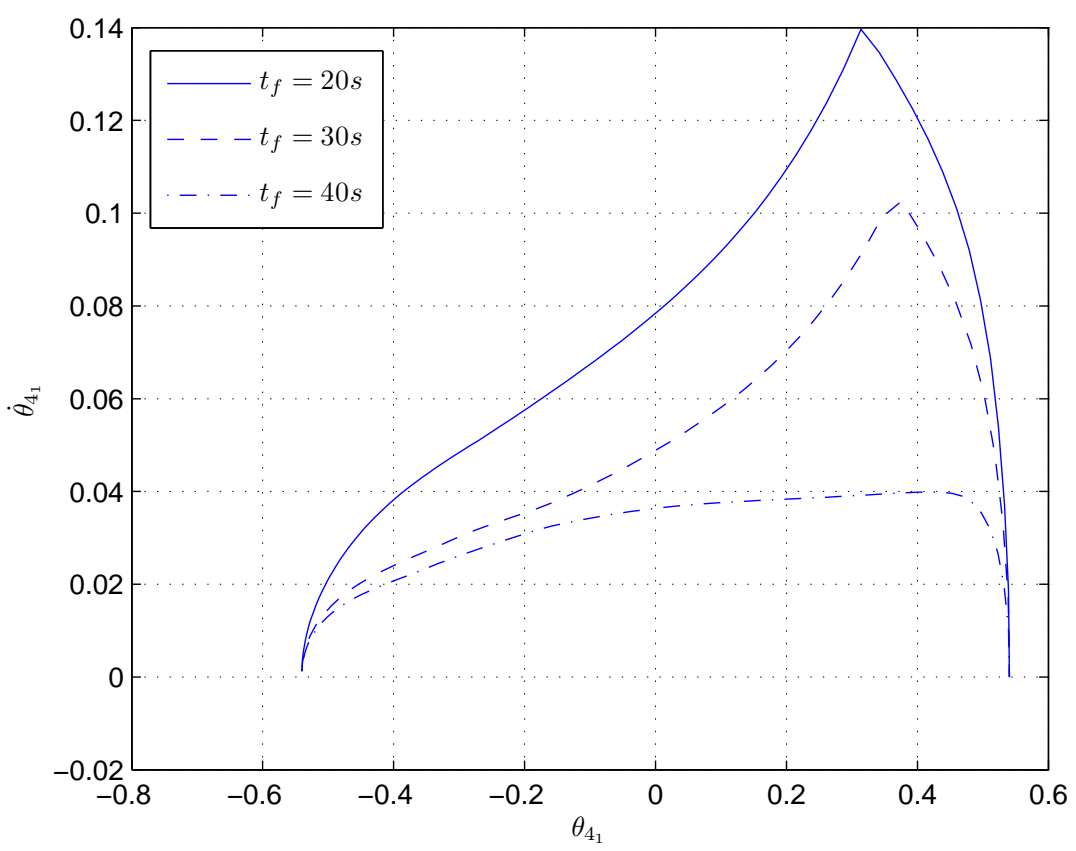

Figura 6.22: Variação da região de deslocamento de $\theta_{4_{1}}$ para diferentes $t_{f}$.

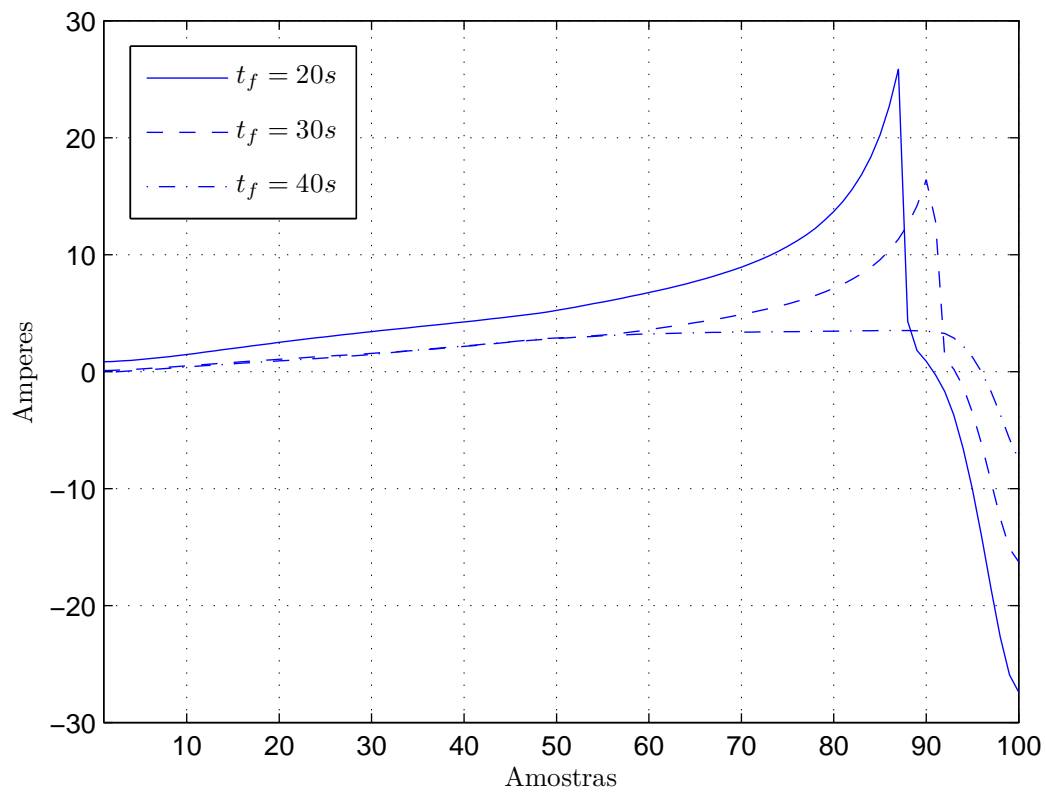

Figura 6.23: Variação da corrente consumida na junta $\theta_{4_{1}}$ para diferentes $t_{f}$. 


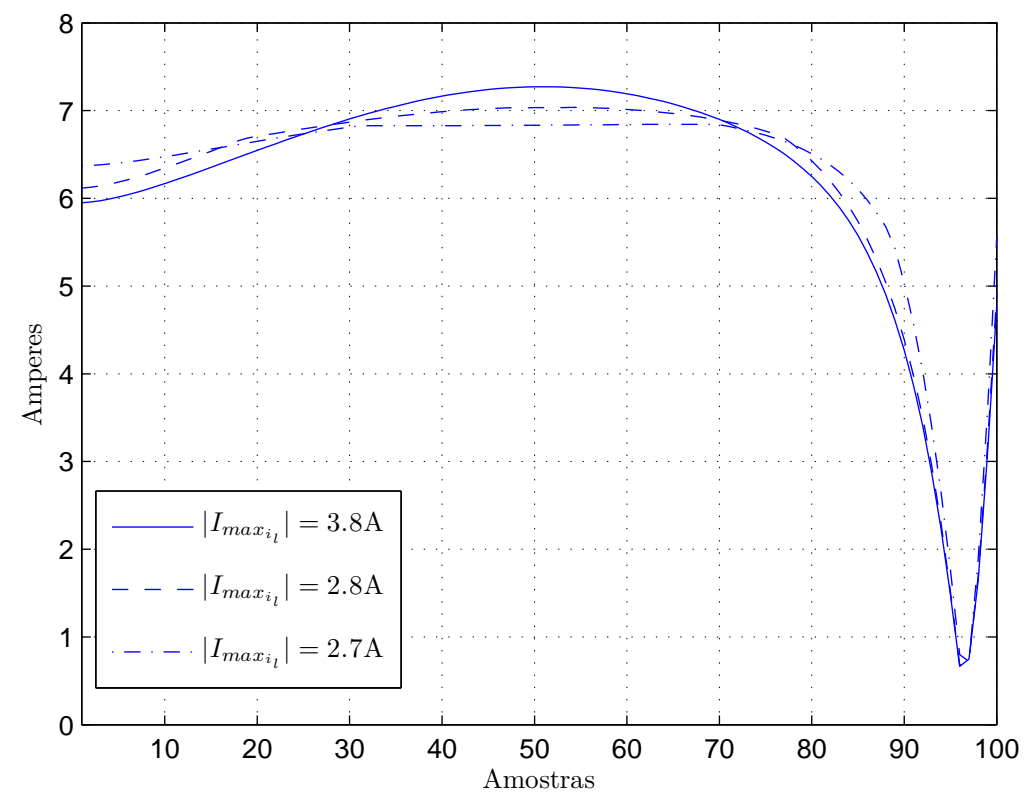

Figura 6.24: Variação da corrente total consumida pelos motores da perna $l=2$.

Tabela 6.4: Resultados da otimização.

\begin{tabular}{ccccccccc}
\hline Etapa & I & \multicolumn{2}{c}{ II } & \multicolumn{3}{c}{ III } & \multicolumn{2}{c}{ IV } \\
Pernas & $l=1,3$ & $l=2$ & $l=4$ & $l=1$ & $l=3$ & $l=2,4$ & $l=1$ & $l=3$ \\
\hline$k$ & 9 & 6 & 6 & 6 & 5 & 20 & 5 & 6 \\
$t_{\text {proc }}[\mathrm{s}]$ & 8.40 & 6.82 & 6.90 & 4.22 & 4.14 & 17.65 & 4.01 & 4.85 \\
Potência $[\mathrm{W}]$ & 154.1 & 14.1 & 13.9 & 11.04 & 14.75 & 114.3 & 11.75 & 11.785 \\
\hline
\end{tabular}

De acordo com a Tabela 6.4 o tempo total de processamento foi de $t_{\text {proc }}=$ $56.99 \mathrm{~s}$, porém, o tempo médio de processamento foi de $\bar{t}_{\text {proc }}=7.12 \mathrm{~s}$.

O deslocamento total da plataforma foi desde a posição ${ }^{O} P(0)$ até a posição ${ }^{O} P(I V)=[0.45,0.68,2.31]$ para uma velocidade média de deslocamento no eixo $Y$ em relação a $\{O\}$ de ${ }^{O} \bar{v}_{Y}=0.0026 m / s$ e um tempo total de ciclo de andadura: $t_{a}=$ $260 s$, onde $t_{a}=t_{f}(I)+t_{f}(I I)+t_{f}(I I I)+t_{f}(I V)$ e $t_{f}(\cdot)$ é o tempo que demora o robô em executar o movimento da etapa $(\cdot)$. Observe-se que na etapa III primeiramente são levantadas as pernas $l=1,3$ para depois movimentar a plataforma.

Note que o objetivo do exemplo não é obter uma grande velocidade de des- 


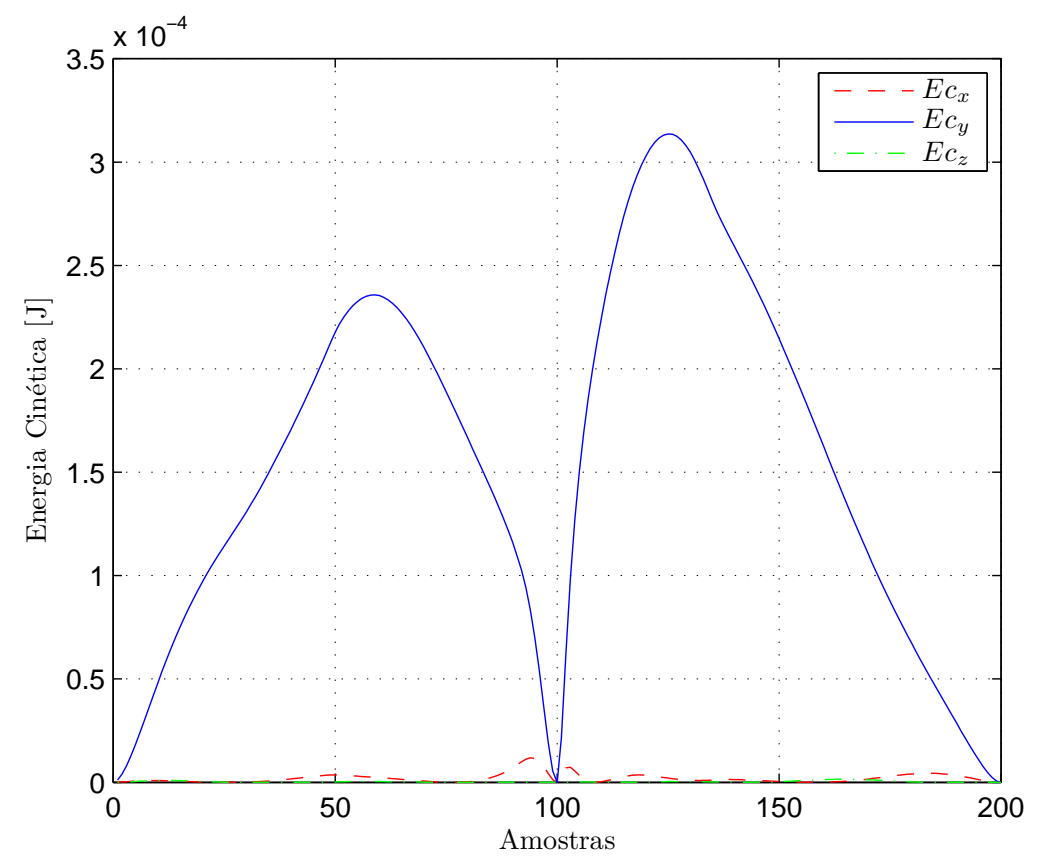

Figura 6.25: Energia cinética ao longo da andadura.

locamento e sim cumprir o conjunto de restrições impostas ao sistema, no relativo ao seu consumo de energia. Para aumentar a velocidade de deslocamento bastaria mudar o conjunto de restrições que limitam o consumo de corrente dos motores.

Um outro dado de interesse é a energia cinética ao longo da andadura. $\mathrm{Na}$ Figura 6.25 são mostrados os valores referentes à energia cinética do robô e, pode ser apreciado que mais do $90 \%$ da energia cinética corresponde ao movimento no eixo $Y$. Este fato evidencia que o movimento gerado pelo algoritmo é coerente com o esperado, pois a maior parte do torque produzido pelos motores é dirigido no sentido do deslocamento e pouca energia é gasta em outras direções. Este padrão energético é similar ao observado na caminhada de camaleões reais (Autumn et al., 2006).

Na implementação física do algoritmo é proposto um controle em camadas (Figura 6.26). Começando deste o topo da pirâmide temos que na primeira camada de controle é escolhida a direção do movimento a seguir e são geradas as coordenadas cartesianas do ponto onde se quer chegar. Na segunda camada de controle é resolvido o problema cinemático inverso e são geradas as restrições cinemáticas e de consumo 


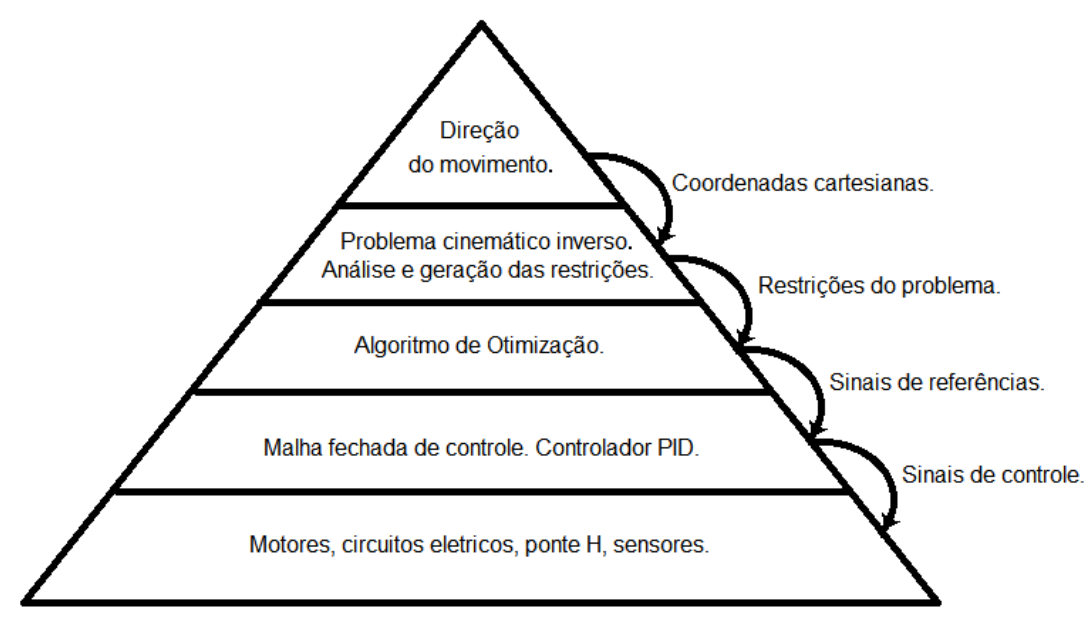

Figura 6.26: Esquema de camadas de controle.

de energia utilizadas no algoritmo de otimização. Resolvido o algoritmo, na seguinte camada, é encontrada uma solução ótima para cada junta ativa e esta é utilizada como sinal de referência na próxima camada de controle. Na quarta camada é implementada estratégia de controle em malha fechada utilizando controladores PID convencionais. Finalmente, a última camada corresponde aos elementos de ação final, motores, sensores e ao acondicionamento dos sinais de controle. 


\section{Capítulo 7}

\section{Conclusões}

Na primeira parte deste trabalho foram desenvolvidos os modelos cinemático e dinâmico do robô Kamambaré. Cada etapa da modelagem foi tratada em detalhe, seguindo uma lógica que começou pela solução dos problemas cinemáticos de posição direto e inverso, continuou com a solução dos problemas cinemáticos de velocidade direto e inverso, prosseguiu com a análise das singularidades e terminou com a solução da dinâmica direta do robô. Para cada uma destas etapas sempre foram adotadas duas abordagens. Na primeira o robô foi tratado com um sistema paralelo com pelo menos uma cadeia cinemática fechada formada pelas pernas que estivessem presas à superfície de apoio, já na segunda abordagem foi modelada a perna do robô no ar como se fosse um manipulador industrial de cadeia aberta com base na plataforma.

Diversos métodos foram utilizados em cada processo de modelagem tentando sempre utilizar aqueles que trouxessem um melhor desempenho de acordo com a topologia modelada e que pudessem ser implementados com facilidade em linguagens de programação de alto nível. Assim, foram utilizados por exemplo, os parâmetros de Denavit-Hartenberg para a solução das cinemáticas de posição diretas da plataforma e da perna, o Principio do Trabalho Virtual ou de D'Alembert para a modelagem dinâmica da plataforma e o método de Newton-Euler para o modelo dinâmico da perna no ar. 
No caso do estudo das singularidades foi utilizado o critério proposto em Goselin e Angeles (1990) para a análise de robôs paralelos.

Finalmente, em relação ao modelo, pode-se dizer que, embora não estejam considerados o atrito entre as juntas e as possíveis deformações dos ligamentos, é um modelo bastante completo que incorpora as grandezas físicas mais importantes para o controle e que pode ser utilizado com facilidade para diversos fins.

Na segunda parte do trabalho foi proposta e demonstrada a convergência de um algoritmo de otimização das perdas elétricas nos motores envolvidos no movimento do robô. O algoritmo visa a minimização da potência elétrica consumida pela resistência de armadura nos motores C.C. usados. Como estratégia de controle foi utilizado o controle independente por junta que trata o torque produzido pelas demais juntas como uma perturbação ao sistema. Além disto, a presença de redução por engrenagens reduz o efeito dinâmico externo sobre o torque gerado pelo motor de cada junta. Devido a esta característica, um problema complexo e difícil de otimizar foi convertido em um PPQ relativamente simples de ser resolvido.

Formulado o PPQ para cada junta independente, o ponto de menor consumo de energia elétrica foi encontrado por meio de um rápido processo iterativo de convergência a um ponto fixo ou mínimo global. Con relação às juntas dependentes, as restrições são satisfeitas de forma indireta variando o tempo de execução dos deslocamentos. Esta abordagem permite satisfazer as restrições impostas ao sistema mediante a solução de poucos PPQs. Outras abordagens que tratassem de forma direta o problema de otimização para cada junta ativa do mecanismo, cairiam em problemas de otimização não lineares, onde novas questões surgiriam na análise dos pontos de partida e na convergência do algoritmo. Além disto, talvez o tempo de processamento resultasse muito maior.

A análise de convergência do algoritmo fornece condições suficientes de convergência de grande utilidade que podem ser aproveitadas desde a etapa de projeto e construção do robô, já que a partir dos parâmetros físicos do sistema é possível 
saber se pode ser implementado ou não este tipo de otimização.

Por último, o algoritmo foi testado utilizando o modelo do Kamambaré obtido na primeira parte do trabalho. Para este fim, foi simulado o deslocamento do robô num ciclo completo de andadura. Desta forma, uma resposta ótima foi obtida tanto para o movimento da plataforma como para o movimento das pernas em separado, enquanto estas estavam no ar. Assim, o algoritmo provou ser eficiente e de rápida convergência nas duas situações consideradas.

É proposta também uma estrutura de camadas, que facilita a hierarquia de controle do robô e que pode ser implementada em qualquer microprocessador convencional. Versões finais do protótipo poderão funcionar de forma autônoma ou semi-autônoma, porém em ambos os casos, as três últimas camadas são internas e independentes do tipo de autonomia do robô.

Como proposta para trabalhos futuros, sugere-se em primeiro lugar, a implementação deste algoritmo no sistema real e a comparação dos resultados obtidos via simulação digital com os reais como forma de validação do modelo. Também sugere-se a utilização do método em outras andaduras. Pode-se ainda, pesquisar o comportamento do robô frente a variações dos conjuntos das juntas ativas e passivas, desde o ponto de vista da otimização, adequar o algoritmo para o controle ótimo do torque da garra e utilizá-lo no planejamento de novas trajetórias em tempo real na evasão de obstáculos. Apesar da rápida convergência do algoritmo, sua implementação digital é pesada, logo seria interessante uma análise detalhada da potência e do tempo consumido durante a execução do mesmo. Finalmente com relação ao modelo, sugere-se ainda a implementação de uma metodologia de modelagem, baseada em manipulação simbólica utilizando o MATLAB, que auxilie na análise e estudo de robôs com pernas. 


\section{Apêndice A}

\section{Velocidades angulares e lineares dos ligamentos}

A seguir são apresentadas as velocidades angulares e lineares dos ligamentos do robô Kamambaré a partir das velocidades lineares e angulares da plataformas relativas ao sistema $\{O\}$. Para a obtenção das mesmas usaram-se as equações:

$$
{ }^{(i+1)_{l}} \omega_{(i+1)_{l}}={ }^{(i+1)_{l}} R_{i_{l}} \cdot{ }^{i_{l}} \omega_{i_{l}}+\dot{\theta}_{(i+1)_{l}} \cdot{ }^{(i+1)_{l}} \hat{Z}_{(i+1)_{l}}
$$

e

$$
{ }^{(i+1)_{l}} v_{(i+1)_{l}}={ }^{(i+1)_{l}} R_{i_{l}}\left({ }^{i_{l}} v_{i_{l}}+{ }^{i_{l}} \omega_{i_{l}} \times{ }^{i_{l}} 0_{(i+1)_{l}}\right) .
$$

Como base do mecanismo foi escolhido o ponto ${ }^{O} E_{l}$. Logo aplicando as equações (A.1) e (A.2) foram obtidas:

$$
\begin{gathered}
v_{2}=\left[\begin{array}{c}
c \theta_{2_{l}}\left(c \theta_{1_{l}} v_{p_{x}}+s \theta_{1_{l}} v_{p_{y}}\right)-s \theta_{2_{l}}\left(v_{p_{z}}-\left(-s \theta_{1_{l}} \omega_{p_{x}}+c \theta_{1_{l}} \omega_{p_{y}}\right) L_{2}\right) \\
-s \theta_{2_{l}}\left(c \theta_{1_{l}} v_{p_{x}}+s \theta_{1_{l}} v_{p_{y}}\right)-c \theta_{2_{l}}\left(v_{p_{z}}-\left(-s \theta_{\dot{1}_{l}} \omega_{p_{x}}+c \theta_{1_{l}} \omega_{p_{y}}\right) L_{2}\right) \\
-s \theta_{1_{l}} v_{p_{x}}+c \theta_{1_{l}} v_{p_{y}}+\left(\omega_{p_{z}}+\dot{\theta}_{1_{l}}\right) L_{2}
\end{array}\right], \\
\omega_{2}=\left[\begin{array}{c}
c \theta_{2_{l}}\left(c \theta_{1_{l}} \omega_{p_{x}}+s \theta_{1_{l}} \omega_{p_{y}}\right)-s \theta_{2_{l}}\left(\omega_{p_{z}}+\dot{\theta}_{1_{l}}\right) \\
-s \theta_{2_{l}}\left(c \theta_{1_{l}} \omega_{p_{x}}+s \theta_{1_{l}} \omega_{p_{y}}\right)-c \theta_{2_{l}}\left(\omega_{p_{z}}+\dot{\theta}_{1_{l}}\right) \\
-s \theta_{1_{l}} \omega_{p_{x}}+c \theta_{1_{l}} \omega_{p_{y}}+\dot{\theta}_{2_{l}}
\end{array}\right], \\
v_{3}=\left[\begin{array}{c}
c \theta_{2_{l} 3_{l}} v_{1_{x_{l}}}-s \theta_{2_{l} 3_{l}}\left(v_{p_{z}}-\omega_{1_{y_{l}}} L_{2}\right)+\left(\omega_{1_{y_{l}}}+\dot{\theta}_{2_{l}}\right) L_{3} \\
-s \theta_{2_{l} 3_{l}} v_{1_{x_{l}}}-c \theta_{2_{l} 3_{l}}\left(v_{p_{z}}-\omega_{1_{y_{l}}} L_{2}\right)+\left(\omega_{1_{y_{l}}}+\dot{\theta}_{2_{l}}\right) L_{3} \\
v_{1_{y_{l}}}+\left(\omega_{p_{z}}+\dot{\theta}_{1_{l}}\right) L_{2}-\left(-s \theta_{2_{l}} \omega_{1_{x_{l}}}-c \theta_{2_{l}}\left(\omega_{p_{z}}+\dot{\theta}_{1_{l}}\right) L_{3}\right)
\end{array}\right],
\end{gathered}
$$




$$
\begin{gathered}
\omega_{3}=\left[\begin{array}{c}
c \theta_{2_{l} 3_{l}} \omega_{1_{x_{l}}}-s \theta_{2_{l} 3_{l}}\left(\omega_{p_{z}}+\dot{\theta}_{1_{l}}\right) \\
-s \theta_{2_{l} 3_{l}} \omega_{1_{x_{l}}}-c \theta_{2_{l} 3_{l}}\left(\omega_{p_{z}}+\dot{\theta}_{1_{l}}\right) \\
\omega_{1_{y_{l}}}+\dot{\theta}_{2_{l}}+\dot{\theta}_{3_{l}}
\end{array}\right] \\
v_{4}=\left[\begin{array}{c}
v_{4_{x_{l}}} \\
v_{4_{y_{l}}} \\
v_{4_{z_{l}}}
\end{array}\right]
\end{gathered}
$$

e

$$
\omega_{4}=\left[\begin{array}{l}
\omega_{4_{x_{l}}} \\
\omega_{4_{y_{l}}} \\
\omega_{4_{z_{l}}}
\end{array}\right]
$$

onde:

$$
\begin{aligned}
& v_{1_{x_{l}}}=c \theta_{1_{l}} v_{p_{x}}+s \theta_{1_{l}} v_{p_{y}} \\
& v_{1_{y_{l}}}=-s \theta_{1_{l}} v_{p_{x}}+c \theta_{1_{l}} v_{p_{y}}, \\
& \omega_{1_{x_{l}}}=c \theta_{1_{l}} \omega_{p_{x}}+s \theta_{1_{l}} \omega_{p_{y}}, \\
& \omega_{1_{x_{l}}}=-s \theta_{1_{l}} \omega_{p_{x}}+c \theta_{1_{l}} \omega_{p_{y}} \\
& v_{4_{x_{l}}}=c \theta_{4_{l}}\left(c \theta_{2_{l} 3_{l}} v_{1_{x_{l}}}-s \theta_{2_{l} 3_{l}}\left(v_{p_{z}}-\omega_{1_{y_{l}}} L_{2}\right)+s \theta_{3}\left(\omega_{1_{y_{l}}}+\dot{\theta_{2_{l}}}\right) L_{3}\right)+ \\
& s \theta_{4_{l}}\left(s \theta_{2_{l} 3_{l}} \omega_{1_{x_{l}}} L_{4}+c \theta_{2_{l} 3_{l}}\left(\omega_{p_{z}}+\dot{\theta}_{1_{l}}\right) L_{4}+v_{1_{y_{l}}}+\left(\omega_{p_{z}}+\dot{\theta}_{1_{l}}\right) L_{2}\right. \\
& \left.+\left(s \theta_{2_{l}} \omega_{1_{x_{l}}}+c \theta_{2_{l}}\left(\omega_{p_{z}}+\dot{\theta}_{1_{l}}\right)\right) L_{3}\right) \text {, } \\
& v_{4_{y_{l}}}=-s \theta_{4_{l}}\left(c \theta_{2_{l} 3_{l}} v_{1_{x_{l}}}-s \theta_{2_{l} 3_{l}}\left(v_{p_{z}}-\omega_{1_{y_{l}}} L_{2}\right)+s \theta_{3}\left(\omega_{1_{y_{l}}}+\dot{\theta_{2_{l}}}\right) L_{3}\right)+ \\
& c \theta_{4_{l}}\left(s \theta_{2_{l} 3_{l}} \omega_{1_{x_{l}}} L_{4}+c \theta_{2_{l} 3_{l}}\left(\omega_{p_{z}}+\dot{\theta}_{1_{l}}\right) L_{4}+v_{1_{y_{l}}}+\left(\omega_{p_{z}}+\theta_{1_{l}}\right) L_{2}\right. \\
& \left.+\left(s \theta_{2_{l}} \omega_{1_{x_{l}}}+c \theta_{2_{l}}\left(\omega_{p_{z}}+\dot{\theta}_{1_{l}}\right)\right) L_{3}\right), \\
& v_{4_{z_{l}}}=s \theta_{2_{l} 3_{l}} v_{1_{x_{l}}}+c \theta_{2_{l} 3_{l}}\left(v_{p_{z}}-\omega_{1_{y_{l}}} L_{2}\right)+\left(\omega_{1_{y_{l}}}+\dot{\theta}_{2_{l}}\right) L_{3}-\left(\omega_{1_{y_{l}}}+\dot{\theta}_{2_{l}}+\dot{\theta}_{3_{l}}\right) L_{4}, \\
& \omega_{4_{x_{l}}}=c \theta_{4_{l}}\left(c \theta_{2_{l} 3_{l}} \omega_{1_{x_{l}}}-s \theta_{2_{l} 3_{l}}\left(\omega_{p_{z}}+\dot{\theta}_{1_{l}}\right)\right)+s \theta_{4_{l}}\left(\omega_{1_{y_{l}}}+\dot{\theta}_{2_{l}}+\dot{\theta}_{3_{l}}\right), \\
& \omega_{4_{y_{l}}}=-s \theta_{4_{l}}\left(c \theta_{2_{l} 3_{l}} \omega_{1_{x_{l}}}-s \theta_{2_{l} 3_{l}}\left(\omega_{p_{z}}+\dot{\theta}_{1_{l}}\right)\right)+c \theta_{4_{l}}\left(\omega_{1_{y_{l}}}+\dot{\theta}_{2_{l}}+\dot{\theta}_{3_{l}}\right)
\end{aligned}
$$


e

$$
\left.\omega_{4_{z_{l}}}=s \theta_{2_{l} 3_{l}} \omega_{1_{x_{l}}}+c \theta_{2_{l} 3_{l}}\left(\omega_{p_{z}}+\dot{\theta}_{1_{l}}\right)+\dot{\theta}_{4_{l}}\right) .
$$

Uma vez calculadas as velocidades angulares e lineares de cada ligamento em função das velocidades angulares e lineares da plataforma é possível calcular os jacobianos dos ligamentos da seguinte forma:

$$
\left[\begin{array}{l}
\vec{v}_{i_{l}} \\
\vec{\omega}_{i_{l}}
\end{array}\right]=J_{i_{l}}\left[\begin{array}{c}
\vec{v}_{P} \\
\vec{\omega}_{P}
\end{array}\right]
$$

Todas as velocidades obtidas são relativas ao sistema inercial $\{O\}$. 


\section{Apêndice B}

\section{Acelerações angulares e lineares dos ligamentos}

Para a obtenção das acelerações angulares e lineares dos ligamentos basta diferenciar com relação ao tempo as equações (A.1) e (A.2). Desta forma as expressões seriam:

${ }^{(i+1)_{l}} \dot{\omega}_{(i+1)_{l}}={ }^{(i+1)_{l}} R_{i_{l}} \cdot{ }^{i_{l}} \dot{\omega}_{i_{l}}+\ddot{\theta}_{(i+1)_{l}} \cdot{ }^{(i+1)_{l}} \hat{Z}_{(i+1)_{l}}+{ }^{(i+1)_{l}} R_{i_{l}} \cdot{ }^{i_{l}} \omega_{i_{l}} \times \dot{\theta}_{(i+1)_{l}} \cdot{ }^{(i+1)_{l}} \hat{Z}_{(i+1)_{l}}$

e

$$
{ }^{(i+1)_{l}} \dot{v}_{(i+1)_{l}}={ }^{(i+1)_{l}} R_{i_{l}}\left[{ }^{i} \dot{\omega}_{i} \times{ }^{i_{l}} 0_{(i+1)_{l}}+{ }^{i_{l}} \omega_{i_{l}} \times\left({ }^{i_{l}} \omega_{i_{l}} \times{ }^{i_{l}} 0_{(i+1)_{l}}\right)+{ }^{i_{l}} \dot{v}_{i_{l}}\right]
$$

Logo, as equações obtidas utilizando (B.1) e (B.2) são as seguintes:

$$
\dot{\omega}_{2_{l}}=\left[\begin{array}{c}
\dot{\omega}_{2_{l_{x}}} \\
\dot{\omega}_{2_{l_{y}}} \\
\dot{\omega}_{2_{l_{z}}}
\end{array}\right]
$$




$$
\begin{aligned}
& \dot{\omega}_{2_{l_{x}}}=c \theta_{2_{l}}\left(c \theta_{1_{l}} \dot{\omega}_{p_{x}}+s \theta_{1_{l}} \dot{\omega}_{p_{y}}+\left(\omega_{1_{y}}\right) \dot{\theta}_{1_{l}}\right)-s \theta_{2_{l}}\left(\dot{\omega}_{p_{z}}+\ddot{\theta}_{1_{l}}\right)+\left(-s \theta_{2_{l}}\left(\omega_{1_{x}}\right)\right. \\
& \left.-c \theta_{2_{l}}\left(\omega_{p_{z}}+\dot{\theta}_{1_{l}}\right)\right) \dot{\theta}_{2_{l}} \\
& \dot{\omega}_{2_{l y}}=-s \theta_{2_{l}}\left(c \theta_{1_{l}} \dot{\omega}_{p_{x}}+s \theta_{1_{l}} \dot{\omega}_{p_{y}}+\left(\omega_{1_{y}}\right) \dot{\theta}_{1_{l}}\right)-c \theta_{2_{l}}\left(\dot{\omega}_{p_{z}}+\ddot{\theta}_{1_{l}}\right)-\left(c \theta_{2_{l}}\left(\omega_{1_{x}}\right)\right. \\
& \left.-s \theta_{2_{l}}\left(\omega_{p_{z}}+\dot{\theta}_{1_{l}}\right)\right) \dot{\theta}_{2_{l}}, \\
& \dot{\omega}_{l_{z}}=-s \theta_{1_{l}} \dot{\omega}_{p_{x}}+c \theta_{1_{l}} \dot{\omega}_{p_{y}}-\left(\omega_{1_{x}}\right) \dot{\theta}_{1_{l}}+\ddot{\theta}_{2_{l}}, \\
& \dot{\omega}_{3_{l}}=\left[\begin{array}{c}
\dot{\omega}_{3_{l_{x}}} \\
\dot{\omega}_{3_{l_{y}}} \\
\dot{\omega}_{3_{l_{z}}}
\end{array}\right] \text {, } \\
& \dot{\omega}_{3_{l_{x}}}=c \theta_{3_{l}}\left(c \theta_{2_{l}}\left(c \theta_{1_{l}} \dot{\omega}_{p_{x}}+s \theta_{1_{l}} \dot{\omega}_{p_{y}}+\left(\omega_{1_{y}}\right) \dot{\theta}_{1_{l}}\right)-s \theta_{2_{l}}\left(\dot{\omega}_{p_{z}}+\ddot{\theta}_{1_{l}}\right)+\left(-s \theta_{2_{l}}\left(\omega_{1_{x}}\right)\right.\right. \\
& \left.\left.-c \theta_{2_{l}}\left(\omega_{p_{z}}+\dot{\theta}_{1_{l}}\right)\right) \dot{\theta}_{2_{l}}\right)+s \theta_{3_{l}}\left(-s \theta_{2_{l}}\left(c \theta_{1_{l}} \dot{\omega}_{p_{x}}+s \theta_{1_{l}} \dot{\omega}_{p_{y}}+\left(\omega_{1_{y}}\right) \dot{\theta}_{1_{l}}\right)\right. \\
& \left.-c \theta_{2_{l}}\left(\dot{\omega}_{p_{z}}+\ddot{\theta}_{1_{l}}\right)-\left(c \theta_{2_{l}}\left(\omega_{1_{x}}\right)-s \theta_{2_{l}}\left(\omega_{p_{z}}+\dot{\theta}_{1_{l}}\right)\right) \dot{\theta}_{2_{l}}\right)+\left(-s \theta_{3_{l}}\left(c \theta_{2_{l}}\left(\omega_{1_{x}}\right)\right.\right. \\
& \left.\left.-s \theta_{2_{l}}\left(\omega_{p_{z}}+\dot{\theta}_{1_{l}}\right)\right)+c \theta_{3_{l}}\left(-s \theta_{2_{l}}\left(\omega_{1_{x}}\right)-c \theta_{2_{l}}\left(\omega_{p_{z}}+\dot{\theta}_{1_{l}}\right)\right)\right) \dot{\theta}_{3_{l}}, \\
& \dot{\omega}_{3_{l y}}=-s \theta_{3_{l}}\left(c \theta_{2_{l}}\left(c \theta_{1_{l}} \dot{\omega}_{p_{x}}+s \theta_{1_{l}} \dot{\omega}_{p_{y}}+\left(\omega_{1_{y}}\right) \dot{\theta}_{1_{l}}\right)-s \theta_{2_{l}}\left(\dot{\omega}_{p_{z}}+\ddot{\theta}_{1_{l}}\right)+\left(-s \theta_{2_{l}}\left(\omega_{1_{x}}\right)\right.\right. \\
& \left.\left.-c \theta_{2_{l}}\left(\omega_{p_{z}}+\dot{\theta}_{1_{l}}\right)\right) \dot{\theta}_{2_{l}}\right)+c \theta_{3_{l}}\left(-s \theta_{2_{l}}\left(c \theta_{1_{l}} \dot{\omega}_{p_{x}}+s \theta_{1_{l}} \dot{\omega}_{p_{y}}+\left(\omega_{1_{y}}\right) \dot{\theta}_{1_{l}}\right)\right. \\
& \left.-c \theta_{2_{l}}\left(\dot{\omega}_{p_{z}}+\ddot{\theta}_{1_{l}}\right)-\left(c \theta_{2_{l}}\left(\omega_{1_{x}}\right)-s \theta_{2_{l}}\left(\omega_{p_{z}}+\dot{\theta}_{1_{l}}\right)\right) \dot{\theta}_{2_{l}}\right)-\left(c \theta _ { 3 _ { l } } \left(c \theta_{2_{l}}\left(\omega_{1_{x}}\right)\right.\right. \\
& \left.\left.-s \theta_{2_{l}}\left(\omega_{p_{z}}+\dot{\theta}_{1_{l}}\right)\right)+s \theta_{3_{l}}\left(-s \theta_{2_{l}}\left(\omega_{1_{x}}\right)-c \theta_{2_{l}}\left(\omega_{p_{z}}+\dot{\theta}_{1_{l}}\right)\right)\right) \dot{\theta}_{3_{l}}, \\
& \dot{\omega}_{3_{l z}}=-s \theta_{1_{l}} \dot{\omega}_{p_{x}}+c \theta_{1_{l}} \dot{\omega}_{p_{y}}-\left(\omega_{1_{x}}\right) \dot{\theta}_{1_{l}}+\ddot{\theta}_{2_{l}}+\ddot{\theta}_{3_{l}}, \\
& \dot{\omega}_{4_{l}}=\left[\begin{array}{c}
\dot{\omega}_{4_{l_{x}}} \\
\dot{\omega}_{4_{l_{y}}} \\
\dot{\omega}_{4_{l_{z}}}
\end{array}\right],
\end{aligned}
$$




$$
\begin{aligned}
& \dot{\omega}_{4_{l x}}=c \theta_{4_{l}}\left(c \theta _ { 3 _ { l } } \left(c \theta_{2_{l}}\left(c \theta_{1_{l}} \dot{\omega}_{p_{x}}+s \theta_{1_{l}} \dot{\omega}_{p_{y}}+\left(\omega_{1_{y}}\right) \dot{\theta}_{1_{l}}\right)-s \theta_{2_{l}}\left(\dot{\omega}_{p_{z}}+\ddot{\theta}_{1_{l}}\right)\right.\right. \\
& \left.+\left(-s \theta_{2_{l}}\left(\omega_{1_{x}}\right)-c \theta_{2_{l}}\left(\omega_{p_{z}}+\dot{\theta}_{1_{l}}\right)\right) \dot{\theta}_{2_{l}}\right)+s \theta_{3_{l}}\left(-s \theta_{2_{l}}\left(c \theta_{1_{l}} \dot{\omega}_{p_{x}}+s \theta_{1_{l}} \dot{\omega}_{p_{y}}\right.\right. \\
& \left.\left.+\left(\omega_{1_{y}}\right) \dot{\theta}_{1_{l}}\right)-c \theta_{2_{l}}\left(\dot{\omega}_{p_{z}}+\ddot{\theta}_{1_{l}}\right)-\left(c \theta_{2_{l}}\left(\omega_{1_{x}}\right)-s \theta_{2_{l}}\left(\omega_{p_{z}}+\dot{\theta}_{1_{l}}\right)\right) \dot{\theta}_{2_{l}}\right) \\
& +\left(-s \theta_{3_{l}}\left(c \theta_{2_{l}}\left(\omega_{1_{x}}\right)-s \theta_{2_{l}}\left(\omega_{p_{z}}+\dot{\theta}_{1_{l}}\right)\right)+c \theta_{3_{l}}\left(-s \theta_{2_{l}}\left(\omega_{1_{x}}\right)-c \theta_{2_{l}}\left(\omega_{p_{z}}\right.\right.\right. \\
& \left.\left.\left.\left.+\dot{\theta}_{1_{l}}\right)\right)\right) \dot{\theta}_{3_{l}}\right)+s \theta_{4_{l}}\left(-s \theta_{1_{l}} \dot{\omega}_{p_{x}}+c \theta_{1_{l}} \dot{\omega}_{p_{y}}-\left(\omega_{1_{x}}\right) \dot{\theta}_{1_{l}}\right. \\
& \left.+\ddot{\theta}_{2_{l}}+\ddot{\theta}_{3_{l}}\right)+\left(-s \theta_{4_{l}}\left(c \theta_{3_{l}}\left(c \theta_{2_{l}}\left(\omega_{1_{x}}\right)-s \theta_{2_{l}}\left(\omega_{p_{z}}+\dot{\theta}_{1_{l}}\right)\right)+s \theta_{3_{l}}\left(-s \theta_{2_{l}}\left(\omega_{1_{x}}\right)\right.\right.\right. \\
& \left.\left.\left.-c \theta_{2_{l}}\left(\omega_{p_{z}}+\dot{\theta}_{1_{l}}\right)\right)\right)+c \theta_{4_{l}}\left(\omega_{1_{y}}+\dot{\theta}_{2_{l}}+\dot{\theta}_{3_{l}}\right)\right) \dot{\theta}_{4_{l}}, \\
& \dot{\omega}_{4_{l_{y}}}=-s \theta_{4_{l}}\left(c \theta _ { 3 _ { l } } \left(c \theta_{2_{l}}\left(c \theta_{1_{l}} \dot{\omega}_{p_{x}}+s \theta_{1_{l}} \dot{\omega}_{p_{y}}+\left(\omega_{1_{y}}\right) \dot{\theta}_{1_{l}}\right)-s \theta_{2_{l}}\left(\dot{\omega}_{p_{z}}+\ddot{\theta}_{1_{l}}\right)\right.\right. \\
& \left.+\left(-s \theta_{2_{l}}\left(\omega_{1_{x}}\right)-c \theta_{2_{l}}\left(\omega_{p_{z}}+\dot{\theta}_{1_{l}}\right)\right) \dot{\theta}_{2_{l}}\right)+s \theta_{3_{l}}\left(-s \theta_{2_{l}}\left(c \theta_{1_{l}} \dot{\omega}_{p_{x}}+s \theta_{1_{l}} \dot{\omega}_{p_{y}}\right.\right. \\
& \left.\left.+\left(\omega_{1_{y}}\right) \dot{\theta}_{1_{l}}\right)-c \theta_{2_{l}}\left(\dot{\omega}_{p_{z}}+\ddot{\theta}_{1_{l}}\right)-\left(c \theta_{2_{l}}\left(\omega_{1_{x}}\right)-s \theta_{2_{l}}\left(\omega_{p_{z}}+\dot{\theta}_{1_{l}}\right)\right) \dot{\theta}_{2_{l}}\right) \\
& \left.+\left(-s \theta_{3_{l}}\left(c \theta_{2_{l}}\left(\omega_{1_{x}}\right)-s \theta_{2_{l}}\left(\omega_{p_{z}}+\dot{\theta}_{1_{l}}\right)\right)+c \theta_{3_{l}}\left(-s \theta_{2_{l}}\left(\omega_{1_{x}}\right)-c \theta_{2_{l}}\left(\omega_{p_{z}}+\dot{\theta}_{1_{l}}\right)\right)\right) \dot{\theta}_{3_{l}}\right) \\
& +c \theta_{4_{l}}\left(-s \theta_{1_{l}} \dot{\omega}_{p_{x}}+c \theta_{1_{l}} \dot{\omega}_{p_{y}}-\left(\omega_{1_{x}}\right) \dot{\theta}_{1_{l}}+\ddot{\theta}_{2_{l}}+\ddot{\theta}_{3_{l}}\right)-\left(c \theta _ { 4 _ { l } } \left(c \theta _ { 3 _ { l } } \left(c \theta_{2_{l}}\left(\omega_{1_{x}}\right)\right.\right.\right. \\
& \left.\left.-s \theta_{2_{l}}\left(\omega_{p_{z}}+\dot{\theta}_{1_{l}}\right)\right)+s \theta_{3_{l}}\left(-s \theta_{2_{l}}\left(\omega_{1_{x}}\right)-c \theta_{2_{l}}\left(\omega_{p_{z}}+\dot{\theta}_{1_{l}}\right)\right)\right)+s \theta_{4_{l}}\left(\omega_{1_{y}}+\dot{\theta}_{2_{l}}\right. \\
& \left.\left.+\dot{\theta}_{3_{l}}\right)\right) \dot{\theta}_{4_{l}}, \\
& \dot{\omega}_{4_{l z}}=s \theta_{3_{l}}\left(c \theta_{2_{l}}\left(c \theta_{1_{l}} \dot{\omega}_{p_{x}}+s \theta_{1_{l}} \dot{\omega}_{p_{y}}+\left(\omega_{1_{y}}\right) \dot{\theta}_{1_{l}}\right)-s \theta_{2_{l}}\left(\dot{\omega}_{p_{z}}+\ddot{\theta}_{1_{l}}\right)+\left(-s \theta_{2_{l}}\left(\omega_{1_{x}}\right)\right.\right. \\
& \left.\left.-c \theta_{2_{l}}\left(\omega_{p_{z}}+\dot{\theta}_{1_{l}}\right)\right) \dot{\theta}_{2_{l}}\right)-c \theta_{3_{l}}\left(-s \theta_{2_{l}}\left(c \theta_{1_{l}} \dot{\omega}_{p_{x}}+s \theta_{1_{l}} \dot{\omega}_{p_{y}}+\left(\omega_{1_{y}}\right) \dot{\theta}_{1_{l}}\right)-c \theta_{2_{l}}\left(\dot{\omega}_{p_{z}}\right.\right. \\
& \left.\left.+\ddot{\theta}_{1_{l}}\right)-\left(c \theta_{2_{l}}\left(\omega_{1_{x}}\right)-s \theta_{2_{l}}\left(\omega_{p_{z}}+\dot{\theta}_{1_{l}}\right)\right) \dot{\theta}_{2_{l}}\right)+\left(c \theta_{3_{l}}\left(c \theta_{2_{l}}\left(\omega_{1_{x}}\right)-s \theta_{2_{l}}\left(\omega_{p_{z}}+\dot{\theta}_{1_{l}}\right)\right)\right. \\
& \left.+s \theta_{3_{l}}\left(-s \theta_{2_{l}}\left(\omega_{1_{x}}\right)-c \theta_{2_{l}}\left(\omega_{p_{z}}+\dot{\theta}_{1_{l}}\right)\right)\right) \dot{\theta}_{3_{l}}+\ddot{\theta}_{4_{l}}, \\
& \dot{v}_{2_{l}}=\left[\begin{array}{l}
\dot{v}_{2_{l_{x}}} \\
\dot{v}_{2_{l_{y}}} \\
\dot{v}_{2_{l_{z}}}
\end{array}\right], \\
& \dot{v}_{2_{l_{x}}}=c \theta_{2_{l}}\left(-\left(\omega_{1_{y}}\right)^{2} L_{2}-\left(\omega_{p_{z}}+\dot{\theta}_{1_{l}}\right)^{2} L_{2}+c \theta_{1_{l}} \dot{v}_{p_{x}}+s \theta_{1_{l}}\left(\dot{v}_{p_{y}}-g\right)\right)-s \theta_{2_{l}}\left(-\left(-s \theta_{1_{l}} \dot{\omega}_{p_{x}}\right.\right. \\
& \left.\left.+c \theta_{1_{l}} \dot{\omega}_{p_{y}}-\left(\omega_{1_{x}}\right) \dot{\theta}_{1_{l}}\right) L_{2}+\left(\omega_{1_{x}}\right)\left(\omega_{p_{z}}+\dot{\theta}_{1_{l}}\right) L_{2}+\dot{v}_{p_{z}}\right)
\end{aligned}
$$




$$
\begin{aligned}
& \dot{v}_{2_{l_{y}}}=-s \theta_{2_{l}}\left(-\left(\omega_{1_{y}}\right)^{2} L_{2}-\left(\omega_{p_{z}}+\dot{\theta}_{1_{l}}\right)^{2} L_{2}+c \theta_{1_{l}} \dot{v}_{p_{x}}+s \theta_{1_{l}}\left(\dot{v}_{p_{y}}-g\right)\right) \\
& -c \theta_{2_{l}}\left(-\left(-s \theta_{1_{l}} \dot{\omega}_{p_{x}}+c \theta_{1_{l}} \dot{\omega}_{p_{y}}-\left(\omega_{1_{x}}\right) \dot{\theta}_{1_{l}}\right) L_{2}+\left(\omega_{1_{x}}\right)\left(\omega_{p_{z}}+\dot{\theta}_{1_{l}}\right) L_{2}+\dot{v}_{p_{z}}\right), \\
& \dot{v}_{2_{z}}=\left(\dot{\omega}_{p_{z}}+\ddot{\theta}_{1_{l}}\right) L_{2}+\left(\omega_{1_{x}}\right)\left(\omega_{1_{y}}\right) L_{2}-s \theta_{1_{l}} \dot{v}_{p_{x}}+c \theta_{1_{l}}\left(\dot{v}_{p_{y}}-g\right) \\
& \dot{v}_{3_{l}}=\left[\begin{array}{l}
\dot{v}_{3_{l_{x}}} \\
\dot{v}_{3_{l_{y}}} \\
\dot{v}_{3_{l_{z}}}
\end{array}\right], \\
& \dot{v}_{3_{l_{x}}}=c \theta_{3_{l}}\left(-\left(-s \theta_{2_{l}}\left(\omega_{1_{x}}\right)-c \theta_{2_{l}}\left(\omega_{p_{z}}+\dot{\theta}_{1_{l}}\right)\right)^{2} L_{3}-\left(\omega_{1_{y}}+\dot{\theta}_{2_{l}}\right)^{2} L_{3}+c \theta_{2_{l}}\left(-\left(\omega_{1_{y}}\right)^{2} L_{2}\right.\right. \\
& \left.-\left(\omega_{p_{z}}+\dot{\theta}_{1_{l}}\right)^{2} L_{2}+c \theta_{1_{l}} \dot{v}_{p_{x}}+s \theta_{1_{l}}\left(\dot{v}_{p_{y}}-g\right)\right)-s \theta_{2_{l}}\left(-\left(-s \theta_{1_{l}} \dot{\omega}_{p_{x}}+c \theta_{1_{l}} \dot{\omega}_{p_{y}}\right.\right. \\
& \left.\left.\left.-\left(\omega_{1_{x}}\right) \dot{\theta}_{1_{l}}\right) L_{2}+\left(\omega_{1_{x}}\right)\left(\omega_{p_{z}}+\dot{\theta}_{1_{l}}\right) L_{2}+\dot{v}_{p_{z}}\right)\right)+s \theta_{3_{l}}\left(\left(-s \theta_{1_{l}} \dot{\omega}_{p_{x}}+c \theta_{1_{l}} \dot{\omega}_{p_{y}}\right.\right. \\
& \left.-\left(\omega_{1_{x}}\right) \dot{\theta}_{1_{l}}+\ddot{\theta}_{2_{l}}\right) L_{3}+\left(c \theta_{2_{l}}\left(\omega_{1_{x}}\right)-s \theta_{2_{l}}\left(\omega_{p_{z}}+\dot{\theta}_{1_{l}}\right)\right)\left(-s \theta_{2_{l}}\left(\omega_{1_{x}}\right)-\right. \\
& \left.c \theta_{2_{l}}\left(\omega_{p_{z}}+\dot{\theta}_{1_{l}}\right)\right) L_{3}-s \theta_{2_{l}}\left(-\left(\omega_{1_{y}}\right)^{2} L_{2}-\left(\omega_{p_{z}}+\dot{\theta}_{1_{l}}\right)^{2} L_{2}+c \theta_{1_{l}} \dot{v}_{p_{x}}+s \theta_{1_{l}}\left(\dot{v}_{p_{y}}-g\right)\right) \\
& \left.-c \theta_{2_{l}}\left(-\left(-s \theta_{1_{l}} \dot{\omega}_{p_{x}}+c \theta_{1_{l}} \dot{\omega}_{p_{y}}-\left(\omega_{1_{x}}\right) \dot{\theta}_{1_{l}}\right) L_{2}+\left(\omega_{1_{x}}\right)\left(\omega_{p_{z}}+\dot{\theta}_{1_{l}}\right) L_{2}+\dot{v}_{p_{z}}\right)\right), \\
& \dot{v}_{3_{l_{y}}}=-s \theta_{3_{l}}\left(-\left(-s \theta_{2_{l}}\left(\omega_{1_{x}}\right)-c \theta_{2_{l}}\left(\omega_{p_{z}}+\dot{\theta}_{1_{l}}\right)\right)^{2} L_{3}-\left(\omega_{1_{y}}+\dot{\theta}_{2_{l}}\right)^{2} L_{3}+c \theta_{2_{l}}\left(-\left(\omega_{1_{y}}\right)^{2} L_{2}\right.\right. \\
& \left.-\left(\omega_{p_{z}}+\dot{\theta}_{1_{l}}\right)^{2} L_{2}+c \theta_{1_{l}} \dot{v}_{p_{x}}+s \theta_{1_{l}}\left(\dot{v}_{p_{y}}-g\right)\right)-s \theta_{2_{l}}\left(-\left(-s \theta_{1_{l}} \dot{\omega}_{p_{x}}+c \theta_{1_{l}} \dot{\omega}_{p_{y}}\right.\right. \\
& \left.\left.\left.-\left(\omega_{1_{x}}\right) \dot{\theta}_{1_{l}}\right) L_{2}+\left(\omega_{1_{x}}\right)\left(\omega_{p_{z}}+\dot{\theta}_{1_{l}}\right) L_{2}+\dot{v}_{p_{z}}\right)\right)+c \theta_{3_{l}}\left(\left(-s \theta_{1_{l}} \dot{\omega}_{p_{x}}+c \theta_{1_{l}} \dot{\omega}_{p_{y}}\right.\right. \\
& \left.-\left(\omega_{1_{x}}\right) \dot{\theta}_{1_{l}}+\ddot{\theta}_{2_{l}}\right) L_{3}+\left(c \theta_{2_{l}}\left(\omega_{1_{x}}\right)-s \theta_{2_{l}}\left(\omega_{p_{z}}+\dot{\theta}_{1_{l}}\right)\right)\left(-s \theta_{2_{l}}\left(\omega_{1_{x}}\right)-c \theta_{2_{l}}\left(\omega_{p_{z}}\right.\right. \\
& \left.\left.+\dot{\theta}_{1_{l}}\right)\right) L_{3}-s \theta_{2_{l}}\left(-\left(\omega_{1_{y}}\right)^{2} L_{2}-\left(\omega_{p_{z}}+\dot{\theta}_{1_{l}}\right)^{2} L_{2}+c \theta_{1_{l}} \dot{v}_{p_{x}}+s \theta_{1_{l}}\left(\dot{v}_{p_{y}}-g\right)\right) \\
& \left.-c \theta_{2_{l}}\left(-\left(-s \theta_{1_{l}} \dot{\omega}_{p_{x}}+c \theta_{1_{l}} \dot{\omega}_{p_{y}}-\left(\omega_{1_{x}}\right) \dot{\theta}_{1_{l}}\right) L_{2}+\left(\omega_{1_{x}}\right)\left(\omega_{p_{z}}+\dot{\theta}_{1_{l}}\right) L_{2}+\dot{v}_{p_{z}}\right)\right), \\
& \dot{v}_{3_{l_{z}}}=-\left(-s \theta_{2_{l}}\left(c \theta_{1_{l}} \dot{\omega}_{p_{x}}+s \theta_{1_{l}} \dot{\omega}_{p_{y}}+\left(\omega_{1_{y}}\right) \dot{\theta}_{1_{l}}\right)-c \theta_{2_{l}}\left(\dot{\omega}_{p_{z}}+\ddot{\theta}_{1_{l}}\right)-\left(c \theta_{2_{l}}\left(\omega_{1_{x}}\right)\right.\right. \\
& \left.\left.-s \theta_{2_{l}}\left(\omega_{p_{z}}+\dot{\theta}_{1_{l}}\right)\right) \dot{\theta}_{2_{l}}\right) L_{3}+\left(c \theta_{2_{l}}\left(\omega_{1_{x}}\right)-s \theta_{2_{l}}\left(\omega_{p_{z}}+\dot{\theta}_{1_{l}}\right)\right)\left(\omega_{1_{y}}+\dot{\theta}_{2_{l}}\right) L_{3} \\
& +\left(\dot{\omega}_{p_{z}}+\ddot{\theta}_{1_{l}}\right) L_{2}+\left(\omega_{1_{x}}\right)\left(\omega_{1_{y}}\right) L_{2}-s \theta_{1_{l}} \dot{v}_{p_{x}}+c \theta_{1_{l}}\left(\dot{v}_{p_{y}}-g\right), \\
& \dot{v}_{4_{l}}=\left[\begin{array}{l}
\dot{v}_{4_{l_{x}}} \\
\dot{v}_{4_{l_{y}}} \\
\dot{v}_{4_{l_{z}}}
\end{array}\right],
\end{aligned}
$$




$$
\begin{aligned}
& \dot{v}_{4_{l_{x}}}=c \theta_{4_{l}}\left(-\left(-s \theta_{3_{l}}\left(c \theta_{2_{l}}\left(\omega_{1_{x}}\right)-s \theta_{2_{l}}\left(\omega_{p_{z}}+\dot{\theta}_{1_{l}}\right)\right)+c \theta_{3_{l}}\left(-s \theta_{2_{l}}\left(\omega_{1_{x}}\right)\right.\right.\right. \\
& \left.\left.-c \theta_{2_{l}}\left(\omega_{p_{z}}+\dot{\theta}_{1_{l}}\right)\right)\right)^{2} L_{4}-\left(\omega_{1_{y}}+\dot{\theta}_{2_{l}}+\dot{\theta}_{3_{l}}\right)^{2} L_{4}+c \theta_{3_{l}}\left(-\left(-s \theta_{2_{l}}\left(\omega_{1_{x}}\right)\right.\right. \\
& \left.-c \theta_{2_{l}}\left(\omega_{p_{z}}+\dot{\theta}_{1_{l}}\right)\right)^{2} L_{3}-\left(\omega_{1_{y}}+\dot{\theta}_{2_{l}}\right)^{2} L_{3}+c \theta_{2_{l}}\left(-\left(\omega_{1_{y}}\right)^{2} L_{2}-\left(\omega_{p_{z}}+\dot{\theta}_{1_{l}}\right)^{2} L_{2}\right. \\
& \left.+c \theta_{1_{l}} \dot{v}_{p_{x}}+s \theta_{1_{l}}\left(\dot{v}_{p_{y}}-g\right)\right)-s \theta_{2_{l}}\left(-\left(-s \theta_{1_{l}} \dot{\omega}_{p_{x}}+c \theta_{1_{l}} \dot{\omega}_{p_{y}}-\left(\omega_{1_{x}}\right) \dot{\theta}_{1_{l}}\right) L_{2}\right. \\
& \left.\left.+\left(\omega_{1_{x}}\right)\left(\omega_{p_{z}}+\dot{\theta}_{1_{l}}\right) L_{2}+\dot{v}_{p_{z}}\right)\right)+s \theta_{3_{l}}\left(\left(-s \theta_{1_{l}} \dot{\omega}_{p_{x}}+c \theta_{1_{l}} \dot{\omega}_{p_{y}}-\left(\omega_{1_{x}}\right) \dot{\theta}_{1_{l}}\right.\right. \\
& \left.+\ddot{\theta}_{2_{l}}\right) L_{3}+\left(c \theta_{2_{l}}\left(\omega_{1_{x}}\right)-s \theta_{2_{l}}\left(\omega_{p_{z}}+\dot{\theta}_{1_{l}}\right)\right)\left(-s \theta_{2_{l}}\left(\omega_{1_{x}}\right)-c \theta_{2_{l}}\left(\omega_{p_{z}}+\dot{\theta}_{1_{l}}\right)\right) L_{3} \\
& -s \theta_{2_{l}}\left(-\left(\omega_{1_{y}}\right)^{2} L_{2}-\left(\omega_{p_{z}}+\dot{\theta}_{1_{l}}\right)^{2} L_{2}+c \theta_{1_{l}} \dot{v}_{p_{x}}+s \theta_{1_{l}}\left(\dot{v}_{p_{y}}-g\right)\right) \\
& \left.\left.-c \theta_{2_{l}}\left(-\left(-s \theta_{1_{l}} \dot{\omega}_{p_{x}}+c \theta_{1_{l}} \dot{\omega}_{p_{y}}-\left(\omega_{1_{x}}\right) \dot{\theta}_{1_{l}}\right) L_{2}+\left(\omega_{1_{x}}\right)\left(\omega_{p_{z}}+\dot{\theta}_{1_{l}}\right) L_{2}+\dot{v}_{p_{z}}\right)\right)\right) \\
& +s \theta_{4_{l}}\left(-\left(-s \theta_{3_{l}}\left(c \theta_{2_{l}}\left(c \theta_{1_{l}} \dot{\omega}_{p_{x}}+s \theta_{1_{l}} \dot{\omega}_{p_{y}}+\left(\omega_{1_{y}}\right) \dot{\theta}_{1_{l}}\right)-s \theta_{2_{l}}\left(\dot{\omega}_{p_{z}}+\ddot{\theta}_{1_{l}}\right)\right.\right.\right. \\
& \left.+\left(-s \theta_{2_{l}}\left(\omega_{1_{x}}\right)-c \theta_{2_{l}}\left(\omega_{p_{z}}+\dot{\theta}_{1_{l}}\right)\right) \dot{\theta}_{2_{l}}\right)+c \theta_{3_{l}}\left(-s \theta_{2_{l}}\left(c \theta_{1_{l}} \dot{\omega}_{p_{x}}+s \theta_{1_{l}} \dot{\omega}_{p_{y}}\right.\right. \\
& \left.\left.+\left(\omega_{1_{y}}\right) \dot{\theta}_{1_{l}}\right)-c \theta_{2_{l}}\left(\dot{\omega}_{p_{z}}+\ddot{\theta}_{1_{l}}\right)-\left(c \theta_{2_{l}}\left(\omega_{1_{x}}\right)-s \theta_{2_{l}}\left(\omega_{p_{z}}+\dot{\theta}_{1_{l}}\right)\right) \dot{\theta}_{2_{l}}\right) \\
& -\left(c \theta_{3_{l}}\left(c \theta_{2_{l}}\left(\omega_{1_{x}}\right)-s \theta_{2_{l}}\left(\omega_{p_{z}}+\dot{\theta}_{1_{l}}\right)\right)+s \theta_{3_{l}}\left(-s \theta_{2_{l}}\left(\omega_{1_{x}}\right)-c \theta_{2_{l}}\left(\omega_{p_{z}}+\right.\right.\right. \\
& \left.\left.\left.\left.\dot{\theta}_{1_{l}}\right)\right)\right) \dot{\theta}_{3_{l}}\right) L_{4}+\left(c \theta_{3_{l}}\left(c \theta_{2_{l}}\left(\omega_{1_{x}}\right)-s \theta_{2_{l}}\left(\omega_{p_{z}}+\dot{\theta}_{1_{l}}\right)\right)\right. \\
& \left.+s \theta_{3_{l}}\left(-s \theta_{2_{l}}\left(\omega_{1_{x}}\right)-c \theta_{2_{l}}\left(\omega_{p_{z}}+\dot{\theta}_{1_{l}}\right)\right)\right)\left(\omega_{1_{y}}+\dot{\theta}_{2_{l}}+\dot{\theta}_{3_{l}}\right) L_{4} \\
& -\left(-s \theta_{2_{l}}\left(c \theta_{1_{l}} \dot{\omega}_{p_{x}}+s \theta_{1_{l}} \dot{\omega}_{p_{y}}+\left(\omega_{1_{y}}\right) \dot{\theta}_{1_{l}}\right)-c \theta_{2_{l}}\left(\dot{\omega}_{p_{z}}+\ddot{\theta}_{1_{l}}\right)\right. \\
& \left.-\left(c \theta_{2_{l}}\left(\omega_{1_{x}}\right)-s \theta_{2_{l}}\left(\omega_{p_{z}}+\dot{\theta}_{1_{l}}\right)\right) \dot{\theta}_{2_{l}}\right) L_{3}+\left(c \theta_{2_{l}}\left(\omega_{1_{x}}\right)-s \theta_{2_{l}}\left(\omega_{p_{z}}\right.\right. \\
& \left.\left.\left.+\dot{\theta}_{1_{l}}\right)\right)\left(\omega_{1_{y}}+\dot{\theta}_{2_{l}}\right) L_{3}+\left(\dot{\omega}_{p_{z}}+\ddot{\theta}_{1_{l}}\right) L_{2}+\left(\omega_{1_{x}}\right)\left(\omega_{1_{y}}\right) L_{2}-s \theta_{1_{l}} \dot{v}_{p_{x}}+c \theta_{1_{l}}\left(\dot{v}_{p_{y}}-g\right)\right),
\end{aligned}
$$




$$
\begin{aligned}
& \dot{v}_{4_{l y}}=-s \theta_{4_{l}}\left(-\left(-s \theta_{3_{l}}\left(c \theta_{2_{l}}\left(\omega_{1_{x}}\right)-s \theta_{2_{l}}\left(\omega_{p_{z}}+\dot{\theta}_{1_{l}}\right)\right)+c \theta_{3_{l}}\left(-s \theta_{2_{l}}\left(\omega_{1_{x}}\right)\right.\right.\right. \\
& \left.\left.-c \theta_{2_{l}}\left(\omega_{p_{z}}+\dot{\theta}_{1_{l}}\right)\right)\right)^{2} L_{4}-\left(\omega_{1_{y}}+\dot{\theta}_{2_{l}}+\dot{\theta}_{3_{l}}\right)^{2} L_{4}+c \theta_{3_{l}}\left(-\left(-s \theta_{2_{l}}\left(\omega_{1_{x}}\right)-c \theta_{2_{l}}\left(\omega_{p_{z}}\right.\right.\right. \\
& \left.\left.+\dot{\theta}_{1_{l}}\right)\right)^{2} L_{3}-\left(\omega_{1_{y}}+\dot{\theta}_{2_{l}}\right)^{2} L_{3}+c \theta_{2_{l}}\left(-\left(\omega_{1_{y}}\right)^{2} L_{2}-\left(\omega_{p_{z}}+\dot{\theta}_{1_{l}}\right)^{2} L_{2}\right. \\
& \left.+c \theta_{1_{l}} \dot{v}_{p_{x}}+s \theta_{1_{l}}\left(\dot{v}_{p_{y}}-g\right)\right)-s \theta_{2_{l}}\left(-\left(-s \theta_{1_{l}} \dot{\omega}_{p_{x}}+c \theta_{1_{l}} \dot{\omega}_{p_{y}}\right.\right. \\
& \left.\left.\left.-\left(\omega_{1_{x}}\right) \dot{\theta}_{1_{l}}\right) L_{2}+\left(\omega_{1_{x}}\right)\left(\omega_{p_{z}}+\dot{\theta}_{1_{l}}\right) L_{2}+\dot{v}_{p_{z}}\right)\right)+s \theta_{3_{l}}\left(\left(-s \theta_{1_{l}} \dot{\omega}_{p_{x}}\right.\right. \\
& \left.+c \theta_{1_{l}} \dot{\omega}_{p_{y}}-\left(\omega_{1_{x}}\right) \dot{\theta}_{1_{l}}+\ddot{\theta}_{2_{l}}\right) L_{3}+\left(c \theta_{2_{l}}\left(\omega_{1_{x}}\right)-s \theta_{2_{l}}\left(\omega_{p_{z}}\right.\right. \\
& \left.\left.+\dot{\theta}_{1_{l}}\right)\right)\left(-s \theta_{2_{l}}\left(\omega_{1_{x}}\right)-c \theta_{2_{l}}\left(\omega_{p_{z}}+\dot{\theta}_{1_{l}}\right)\right) L_{3}-s \theta_{2_{l}}\left(-\left(\omega_{1_{y}}\right)^{2} L_{2}-\left(\omega_{p_{z}}\right.\right. \\
& \left.\left.+\dot{\theta}_{1_{l}}\right)^{2} L_{2}+c \theta_{1_{l}} \dot{v}_{p_{x}}+s \theta_{1_{l}}\left(\dot{v}_{p_{y}}-g\right)\right)-c \theta_{2_{l}}\left(-\left(-s \theta_{1_{l}} \dot{\omega}_{p_{x}}+c \theta_{1_{l}} \dot{\omega}_{p_{y}}\right.\right. \\
& \left.\left.\left.\left.-\left(\omega_{1_{x}}\right) \dot{\theta}_{1_{l}}\right) L_{2}+\left(\omega_{1_{x}}\right)\left(\omega_{p_{z}}+\dot{\theta}_{1_{l}}\right) L_{2}+\dot{v}_{p_{z}}\right)\right)\right)+c \theta_{4_{l}}\left(-\left(-s \theta_{3_{l}}\left(c \theta _ { 2 _ { l } } \left(c \theta_{1_{l}} \dot{\omega}_{p_{x}}\right.\right.\right.\right. \\
& \left.+s \theta_{1_{l}} \dot{\omega}_{p_{y}}+\left(\omega_{1_{y}}\right) \dot{\theta}_{1_{l}}\right)-s \theta_{2_{l}}\left(\dot{\omega}_{p_{z}}+\ddot{\theta}_{1_{l}}\right)+\left(-s \theta_{2_{l}}\left(\omega_{1_{x}}\right)\right. \\
& \left.\left.-c \theta_{2_{l}}\left(\omega_{p_{z}}+\dot{\theta}_{1_{l}}\right)\right) \dot{\theta}_{2_{l}}\right)+c \theta_{3_{l}}\left(-s \theta_{2_{l}}\left(c \theta_{1_{l}} \dot{\omega}_{p_{x}}+s \theta_{1_{l}} \dot{\omega}_{p_{y}}\right.\right. \\
& \left.+\left(\omega_{1_{y}}\right) \dot{\theta}_{1_{l}}\right)-c \theta_{2_{l}}\left(\dot{\omega}_{p_{z}}+\ddot{\theta}_{1_{l}}\right)-\left(c \theta_{2_{l}}\left(\omega_{1_{x}}\right)-s \theta_{2_{l}}\left(\omega_{p_{z}}\right.\right. \\
& \left.\left.\left.+\dot{\theta}_{1_{l}}\right)\right) \dot{\theta}_{2_{l}}\right)-\left(c \theta_{3_{l}}\left(c \theta_{2_{l}}\left(\omega_{1_{x}}\right)-s \theta_{2_{l}}\left(\omega_{p_{z}}+\dot{\theta}_{1_{l}}\right)\right)+s \theta_{3_{l}}\left(-s \theta_{2_{l}}\left(\omega_{1_{x}}\right)\right.\right. \\
& \left.\left.\left.-c \theta_{2_{l}}\left(\omega_{p_{z}}+\dot{\theta}_{1_{l}}\right)\right)\right) \dot{\theta}_{3_{l}}\right) L_{4}+\left(c \theta_{3_{l}}\left(c \theta_{2_{l}}\left(\omega_{1_{x}}\right)-s \theta_{2_{l}}\left(\omega_{p_{z}}+\dot{\theta}_{1_{l}}\right)\right)\right. \\
& \left.+s \theta_{3_{l}}\left(-s \theta_{2_{l}}\left(\omega_{1_{x}}\right)-c \theta_{2_{l}}\left(\omega_{p_{z}}+\dot{\theta}_{1_{l}}\right)\right)\right)\left(\omega_{1_{y}}+\dot{\theta}_{2_{l}}+\dot{\theta}_{3_{l}}\right) L_{4} \\
& -\left(-s \theta_{2_{l}}\left(c \theta_{1_{l}} \dot{\omega}_{p_{x}}+s \theta_{1_{l}} \dot{\omega}_{p_{y}}+\left(\omega_{1_{y}}\right) \dot{\theta}_{1_{l}}\right)-c \theta_{2_{l}}\left(\dot{\omega}_{p_{z}}\right.\right. \\
& \left.\left.+\ddot{\theta}_{1_{l}}\right)-\left(c \theta_{2_{l}}\left(\omega_{1_{x}}\right)-s \theta_{2_{l}}\left(\omega_{p_{z}}+\dot{\theta}_{1_{l}}\right)\right) \dot{\theta}_{2_{l}}\right) L_{3}+\left(c \theta_{2_{l}}\left(\omega_{1_{x}}\right)\right. \\
& \left.-s \theta_{2_{l}}\left(\omega_{p_{z}}+\dot{\theta}_{1_{l}}\right)\right)\left(\omega_{1_{y}}+\dot{\theta}_{2_{l}}\right) L_{3}+\left(\dot{\omega}_{p_{z}}+\ddot{\theta}_{1_{l}}\right) L_{2}+\left(\omega_{1_{x}}\right)\left(\omega_{1_{y}}\right) L_{2} \\
& \left.-s \theta_{1_{l}} \dot{v}_{p_{x}}+c \theta_{1_{l}}\left(\dot{v}_{p_{y}}-g\right)\right)
\end{aligned}
$$




$$
\begin{aligned}
\dot{v}_{4_{l_{z}}}= & -\left(-s \theta_{1_{l}} \dot{\omega}_{p_{x}}+c \theta_{1_{l}} \dot{\omega}_{p_{y}}-\left(\omega_{1_{x}}\right) \dot{\theta}_{1_{l}}+\ddot{\theta}_{2_{l}}+\ddot{\theta}_{3_{l}}\right) L_{4}-\left(c \theta _ { 3 _ { l } } \left(c \theta_{2_{l}}\left(\omega_{1_{x}}\right)\right.\right. \\
& \left.\left.-s \theta_{2_{l}}\left(\omega_{p_{z}}+\dot{\theta}_{1_{l}}\right)\right)+s \theta_{3_{l}}\left(-s \theta_{2_{l}}\left(\omega_{1_{x}}\right)-c \theta_{2_{l}}\left(\omega_{p_{z}}+\dot{\theta}_{1_{l}}\right)\right)\right)\left(-s \theta_{3_{l}}\left(c \theta_{2_{l}}\left(\omega_{1_{x}}\right)\right.\right. \\
& \left.\left.-s \theta_{2_{l}}\left(\omega_{p_{z}}+\dot{\theta}_{1_{l}}\right)\right)+c \theta_{3_{l}}\left(-s \theta_{2_{l}}\left(\omega_{1_{x}}\right)-c \theta_{2_{l}}\left(\omega_{p_{z}}+\dot{\theta}_{1_{l}}\right)\right)\right) L_{4}+s \theta_{3_{l}}\left(-\left(-s \theta_{2_{l}}\left(\omega_{1_{x}}\right)\right.\right. \\
& \left.-c \theta_{2_{l}}\left(\omega_{p_{z}}+\dot{\theta}_{1_{l}}\right)\right)^{2} L_{3}-\left(\omega_{1_{y}}+\dot{\theta}_{2_{l}}\right)^{2} L_{3}+c \theta_{2_{l}}\left(-\left(\omega_{1_{y}}\right)^{2} L_{2}-\left(\omega_{p_{z}}+\dot{\theta}_{1_{l}}\right)^{2} L_{2}\right. \\
& \left.+c \theta_{1_{l}} \dot{v}_{p_{x}}+s \theta_{1_{l}}\left(\dot{v}_{p_{y}}-g\right)\right)-s \theta_{2_{l}}\left(-\left(-s \theta_{1_{l}} \dot{\omega}_{p_{x}}+c \theta_{1_{l}} \dot{\omega}_{p_{y}}-\left(\omega_{1_{x}}\right) \dot{\theta}_{1_{l}}\right) L_{2}\right. \\
& \left.\left.+\left(\omega_{1_{x}}\right)\left(\omega_{p_{z}}+\dot{\theta}_{1_{l}}\right) L_{2}+\dot{v}_{p_{z}}\right)\right)-c \theta_{3_{l}}\left(\left(-s \theta_{1_{l}} \dot{\omega}_{p_{x}}+c \theta_{1_{l}} \dot{\omega}_{p_{y}}-\left(\omega_{1_{x}}\right) \dot{\theta}_{1_{l}}\right.\right. \\
& \left.+\ddot{\theta}_{2_{l}}\right) L_{3}+\left(c \theta_{2_{l}}\left(\omega_{1_{x}}\right)-s \theta_{2_{l}}\left(\omega_{p_{z}}+\dot{\theta}_{1_{l}}\right)\right)\left(-s \theta_{2_{l}}\left(\omega_{1_{x}}\right)-c \theta_{2_{l}}\left(\omega_{p_{z}}+\dot{\theta}_{1_{l}}\right)\right) L_{3} \\
& -s \theta_{2_{l}}\left(-\left(\omega_{1_{y}}\right)^{2} L_{2}-\left(\omega_{p_{z}}+\dot{\theta}_{1_{l}}\right)^{2} L_{2}+c \theta_{1_{l}} \dot{v}_{p_{x}}+s \theta_{1_{l}}\left(\dot{v}_{p_{y}}-g\right)\right)-c \theta_{2_{l}}\left(-\left(-s \theta_{1_{l}} \dot{\omega}_{p_{x}}\right.\right. \\
& \left.\left.\left.+c \theta_{1_{l}} \dot{\omega}_{p_{y}}-\left(\omega_{1_{x}}\right) \dot{\theta}_{1_{l}}\right) L_{2}+\left(\omega_{1_{x}}\right)\left(\omega_{p_{z}}+\dot{\theta}_{1_{l}}\right) L_{2}+\dot{v}_{p_{z}}\right)\right) .
\end{aligned}
$$

Da mesma forma que no Apêndice A, todas as acelerações obtidas são relativas ao sistema $\{O\}$. 


\section{Apêndice $\mathrm{C}$}

\section{Variáveis dependentes na etapa I da andadura do robô}

Neste apêndice será resolvido o sistema de equações apresentado no Capítulo 6, tendo em consideração que o conjunto das variáveis independentes é $\mathcal{I}=\left\{\theta_{2_{3}}, \theta_{3_{3}}, \theta_{4_{3}}\right\}$, o das variáveis dependentes é $\mathcal{D}=\left\{\theta_{1_{3}}, \theta_{1_{1}}, \theta_{2_{1}}, \theta_{3_{1}}, \theta_{4_{1}}\right\}$ e as amplitudes de trabalho de cada junta são as mostradas na Tabela C.1.

Tabela C.1: Amplitude de trabalho das juntas do Kamambaré

\begin{tabular}{ccccccccc}
\hline Junta & $\theta_{1_{1}}$ & $\theta_{2_{1}}$ & $\theta_{3_{1}}$ & $\theta_{4_{1}}$ & $\theta_{1_{3}}$ & $\theta_{2_{3}}$ & $\theta_{3_{3}}$ & $\theta_{4_{3}}$ \\
\hline Amplitude & {$[0, \pi]$} & {$\left[-\frac{\pi}{4},-\frac{3 \pi}{4}\right]$} & {$\left[\frac{\pi}{4}, \frac{3 \pi}{4}\right]$} & {$\left[-\frac{\pi}{4}, \frac{\pi}{4}\right]$} & {$[-\pi, 0]$} & {$\left[\frac{\pi}{4}, \frac{3 \pi}{4}\right]$} & {$\left[\frac{\pi}{4}, \frac{3 \pi}{4}\right]$} & {$\left[-\frac{\pi}{4}, \frac{\pi}{4}\right]$} \\
\hline
\end{tabular}

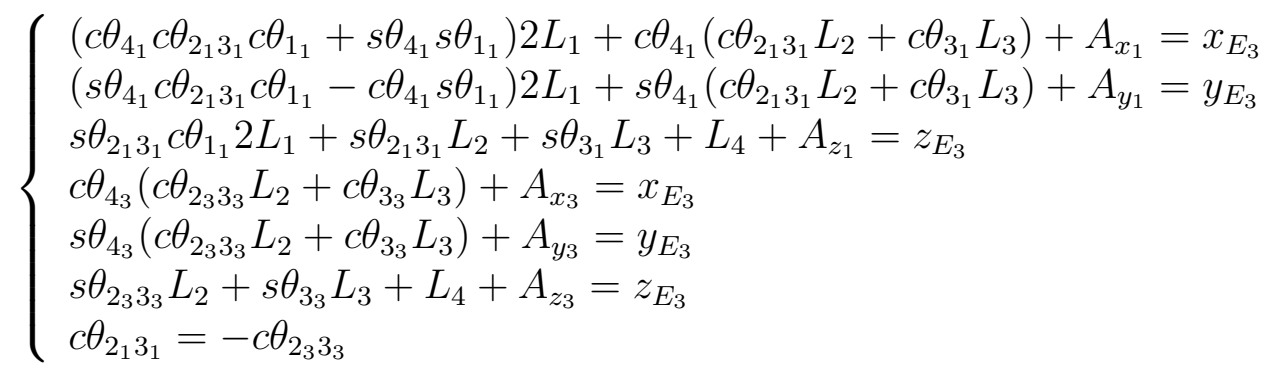

Duas possíveis vias de solução existem para os ângulos $\theta_{1_{1}}$ e $\theta_{3_{1}}$. A primeira via de solução ocorre quando $s \theta_{2_{1} 3_{1}}=s \theta_{2_{3} 3_{3}}=0$, para esta condição temos:

$$
s \theta_{3_{1}}=\frac{z_{E_{3}}-L_{4}-A_{z_{1}}}{L_{3}}
$$


e

$$
c \theta_{1_{1}}=\frac{x_{E_{3}}^{2}+y_{E_{3}}^{2}-4 L_{1}^{2}-\left(L_{2}+c \theta_{3_{1}} L_{3}\right)^{2}}{4 L_{1}^{2}\left(L_{2}+c \theta_{3_{1}} L_{3}\right)^{2}} .
$$

A segunda via de solução ocorre para $s \theta_{2_{1} 3_{1}} \neq 0$. Neste caso os valores relativos ao ângulo $\theta_{3_{1}}$ estão nas raízes reais $\left(r_{1,2,3,4}\right)$ do seguinte polinômio:

$$
p_{4} r^{4}+p_{3} r^{3}+p_{2} r^{2}+p_{1} r+p_{0}=0
$$

onde:

$$
\begin{aligned}
p_{4} & =J^{2}+4 A^{2} B^{2} L_{3}^{4}, \\
p_{3} & =2 J N+8 A B^{2} L_{3}^{3} D, \\
p_{2} & =N^{2}-2 J K-4 A^{2} B^{2} L_{3}^{4}+4 B^{2} L_{3}^{2} D^{2}, \\
p_{1} & =-2 N K-8 A B^{2} L_{3}^{3} D, \\
p_{0} & =K^{2}-4 B^{2} L_{3}^{2} D^{2}, \\
r & =c \theta_{3_{1}}
\end{aligned}
$$

e

$$
\begin{aligned}
A & =-c \theta_{2_{3} 3_{3}}, \\
B & =s \theta_{2_{3} 3_{3}}, \\
E & =B^{2}\left(x_{E_{3}}^{2}+y_{E_{3}}^{2}-4 L_{1}^{2}\right), \\
D & =L_{2}-z_{E_{3}} B, \\
K & =E-A^{2} L_{2}^{2}+D^{2}+L_{3}^{2} B^{2}, \\
J & =2 A L_{2} L_{3}-2 A L_{3} D, \\
N & =2 L_{3}^{2} B^{2} .
\end{aligned}
$$

Logo,

$$
c \theta_{3_{1,2,3,4}}=r_{1,2,3,4}
$$

e

$$
c \theta_{1_{1_{1,2}}}=\frac{A\left(A L_{2}+c \theta_{3_{1}} L_{3}\right) \pm \sqrt{\left(A L_{2}+c \theta_{3_{1}} L_{3}\right)^{2}-B^{2} x_{E_{3}}^{2}+y_{E_{3}}^{2}-4 L_{1}^{2}}}{2 L_{1} B^{2}}
$$


Para a obtenção dos valores da junta $\theta_{2_{1}}$ tem-se:

$$
s \theta_{2_{1,2}}=-A s \theta_{3_{1}} \pm B c \theta_{3_{1}} .
$$

Já para o valor da junta $\theta_{4_{1}}$ é necessário encontrar as raízes reais do polinômio de tercer grau:

$$
q_{2} s^{2}+q_{1} s+q_{0}=0
$$

onde

$$
\begin{aligned}
q_{2} & =C^{2}+4 L_{1}^{2} s \theta_{1_{1}}^{2}, \\
q_{1} & =-2 C y_{E_{3}}, \\
q_{0} & =y_{E_{3}}^{2}-4 L_{1}^{2} s \theta_{1_{1}}^{2}, \\
s & =s \theta_{4_{1}}
\end{aligned}
$$

e

$$
C=2 L_{1} c \theta_{2_{1} 3_{1}} c \theta_{1_{1}}+L_{2} c \theta_{2_{1} 3_{1}}+L_{3} c \theta_{3_{1}}
$$

assim, $s \theta_{4_{1,2,3}}=s_{1,2,3}$.

Por último, o ângulo $\theta_{1_{3}}$ pode ser encontrado utilizando a definição do produto vetorial como:

$$
\theta_{1_{3}}=\arccos \left(-c \theta_{2_{1} 3_{1}}\left(c \theta_{2_{1} 3_{1}} c \theta_{1_{1}} c\left(\theta_{4_{1}}-\theta_{4_{2}}\right)-s \theta_{1_{1}} s\left(\theta_{4_{1}}-\theta_{4_{2}}\right)\right)+c \theta_{1_{1}} s \theta_{2_{1} 3_{1}}^{2}\right)-\pi .
$$

Dentro do conjunto de soluções de C.1 sempre é escolhido aquele subconjunto da solução que esteja mais próximo do estado anterior. 


\section{Apêndice D}

\section{Motor RS-540-12v}

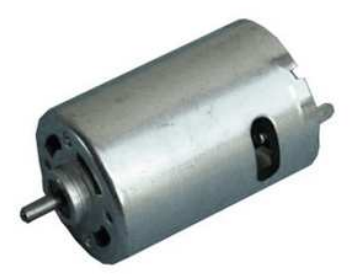

Figura D.1: Motor estilo Mabuchi RS-540 de 3 pólos de armadura. Fonte: TrossenRobotics

Tabela D.1: Características do motor. Fonte: TrossenRobotics Especificações

\begin{tabular}{llll}
\hline \multicolumn{2}{c}{ Desempenho } & \multicolumn{2}{c}{ Físicas } \\
\hline Tensão Nominal & $12 \mathrm{v}$ & Comprimento & $50 \mathrm{~mm}$ \\
RPM sem carga & 16800 & Diâmetro & $35.8 \mathrm{~mm}$ \\
Corrente sem carga & $1 \mathrm{~A}$ & Diâmetro do eixo & $3.2 \mathrm{~mm}$ \\
Torque máximo & $278.8 \mathrm{mN}-\mathrm{m}$ & Comprimento do eixo & $7.6 \mathrm{~mm}$ \\
Corrente máxima & $42 \mathrm{~A}$ & Peso & $153 \mathrm{~g}$ \\
$K_{m}$ & $6.6 \mathrm{mN}-\mathrm{m} / \mathrm{A}$ & & \\
$K_{b}$ & $1400 \mathrm{RPM} / \mathrm{v}$ & & \\
Eficiência & $71 \%$ & & \\
\hline
\end{tabular}




\section{Apêndice E}

\section{Kit universal de redução por engrenagem Tamiya 70103}

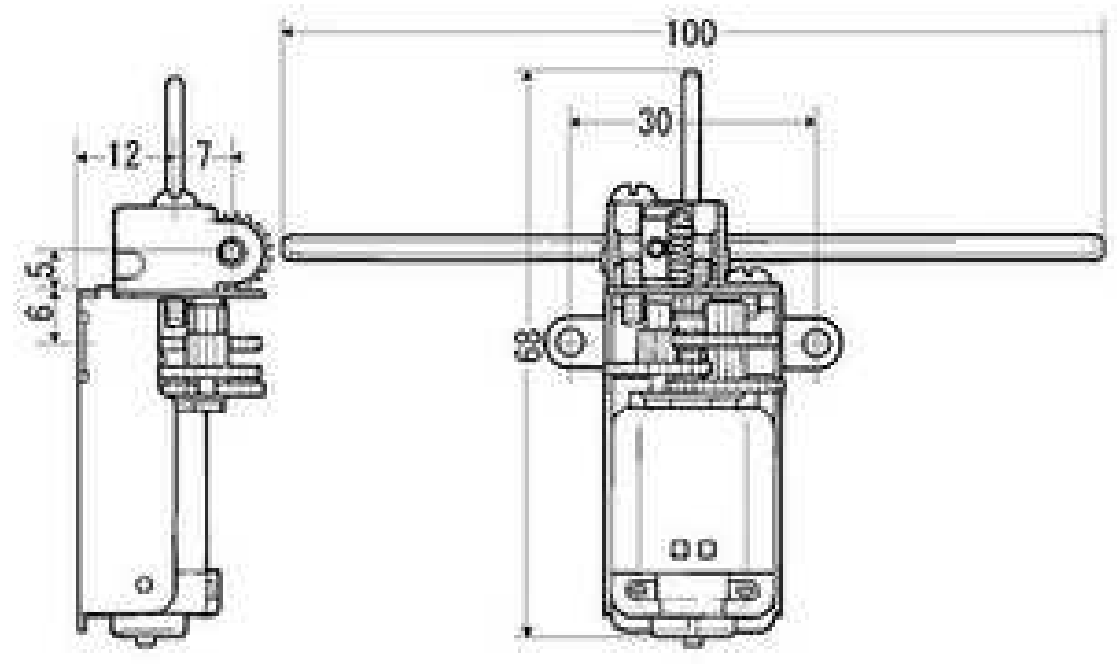

Figura E.1: Redução por engrenagem Tamiya 70103. Fatores de redução de 101:1, 269:1 e 719:1. Fonte: Robotics Electronics 


\section{Apêndice F}

\section{Bateria selada BT-12M14AC}

\begin{tabular}{|c|c|c|}
\hline \multicolumn{3}{|c|}{ PHYSICAL SPECIFICATION } \\
\hline \multicolumn{2}{|c|}{ Nominal Voltage } & $12 \mathrm{~V}$ \\
\hline \multicolumn{2}{|c|}{ Nominal Capacity (20HR) } & $14 \mathrm{AH}$ \\
\hline \multirow{4}{*}{ Dimension } & Length & $151 \pm 2 \mathrm{~mm}(5.94$ inches $)$ \\
\hline & Width & $98 \pm 1 \mathrm{~mm}(3.86$ inches $)$ \\
\hline & Container height & $95 \pm 1 \mathrm{~mm}(3.74$ inches $)$ \\
\hline & Total Height(with terminal) & $99 \pm 2 \mathrm{~mm}$ (3.90inches) \\
\hline \multicolumn{2}{|c|}{ Weight } & Approx $3.94 \mathrm{Kg}(8.69 \mathrm{Ibs})$ \\
\hline \multicolumn{2}{|c|}{ Standard Terminal } & F2 (standard) \\
\hline
\end{tabular}

Figura F.1: Especificações físicas. Fonte: Eletronica-Industrial

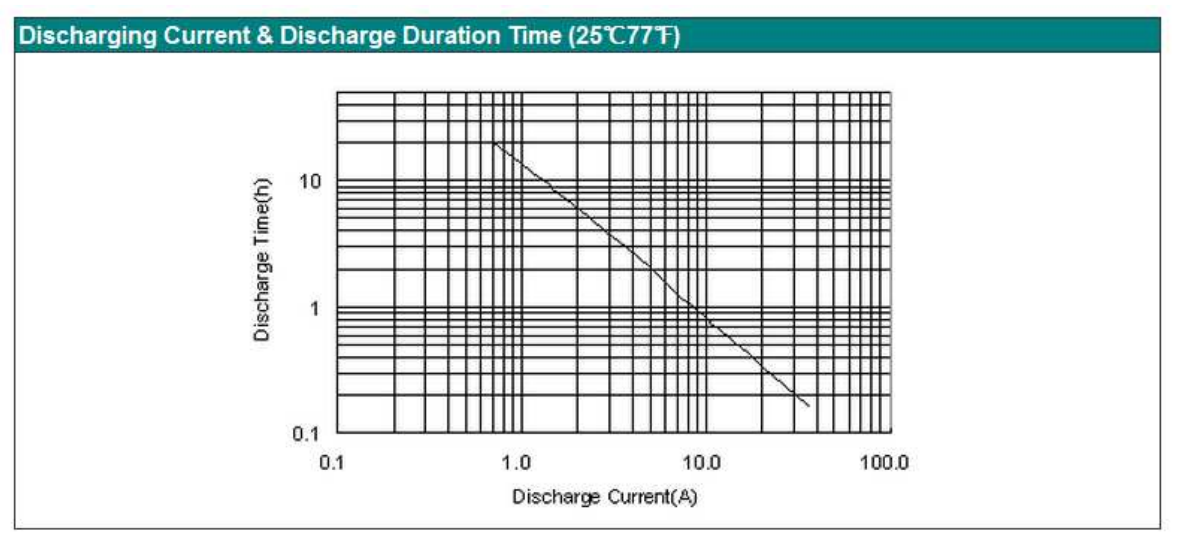

Figura F.2: Tempo de descarga em função do consumo de corrente do circuito. Fonte: Eletronica-Industrial 


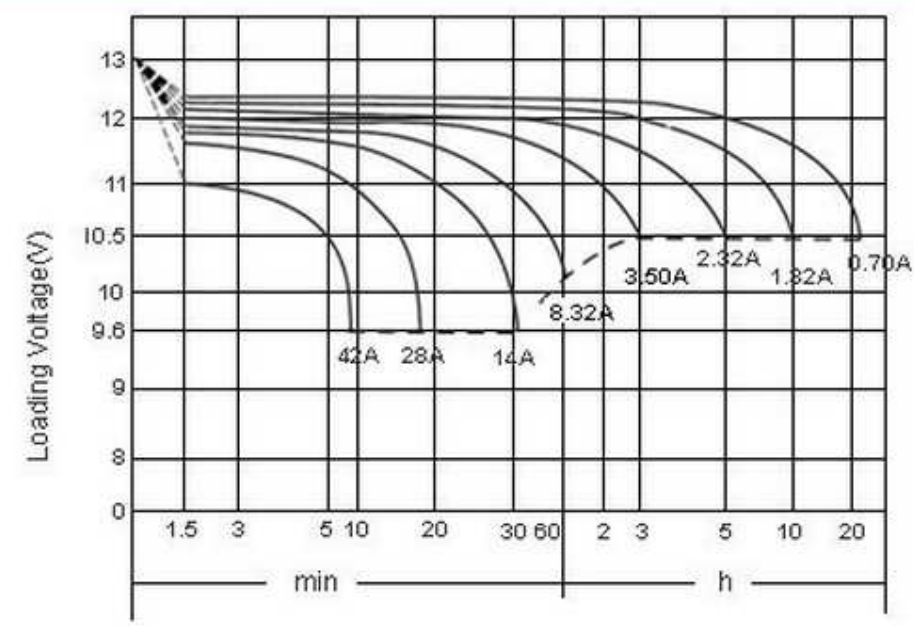

Discharge Time

Figura F.3: Curvas de descarga da bateria. Fonte: Eletronica-Industrial

\begin{tabular}{|c|c|c|c|c|c|}
\hline \multicolumn{6}{|c|}{ ELECTRICAL SPECIFICATION } \\
\hline \multirow{5}{*}{ Rated Capacity } & 20 hour rate $(0.70 \mathrm{~A})$ & $14.0 \mathrm{AH}$ & \multirow{9}{*}{$\begin{array}{l}\text { Constant- } \\
\text { Voltage } \\
\text { Charge }\end{array}$} & \multirow{5}{*}{ Cycle } & \multirow{5}{*}{$\begin{array}{c}\text { Initial Charging Current less than } \\
3.5 \mathrm{~A} . \text { Voltage } 14.5 \mathrm{~V}-15.0 \mathrm{~V} \text { at } \\
25^{\circ} \mathrm{C}\left(77^{\circ} \mathrm{F}\right) \text { Temp. Coefficient - } \\
\text { 30mV/ } \mathrm{C}\end{array}$} \\
\hline & 10 hour rate $(1.32 \mathrm{~A})$ & $13.2 \mathrm{AH}$ & & & \\
\hline & 5 hour rate(2.32A) & 11.6AH & & & \\
\hline & 1 hour rate( $8.32 \mathrm{~A})$ & 8.32AH & & & \\
\hline & 15 minute rate $(23.5 \mathrm{~A})$ & 5.8AH & & & \\
\hline \multirow{3}{*}{$\begin{array}{l}\text { Capacity affected } \\
\text { by Temperature }\end{array}$} & $40^{\circ} \mathrm{C}\left(104^{\circ} \mathrm{F}\right)$ & $103 \%$ & & \multirow{4}{*}{ Standby } & \multirow{4}{*}{$\begin{array}{c}\text { No limit on Initial Charging } \\
\text { Current Voltage } 13.6 \mathrm{~V}-13.8 \mathrm{~V} \text { at } \\
25^{\circ} \mathrm{C}\left(77^{\circ} \mathrm{F}\right) \text { Temp. Coefficient - } \\
18 \mathrm{mV} / \mathrm{C}\end{array}$} \\
\hline & $25^{\circ} \mathrm{C}\left(77^{\circ} \mathrm{F}\right)$ & $100 \%$ & & & \\
\hline & $0^{\circ} \mathrm{C}\left(32^{\circ} \mathrm{F}\right)$ & $86 \%$ & & & \\
\hline Internal Resistance & \multicolumn{2}{|l|}{ Fully charged battery ( 2} & & & \\
\hline
\end{tabular}

Figura F.4: Especificações elétricas. Fonte: Eletronica-Industrial 


\section{Apêndice G}

\section{Sequência de movimentos do robô}
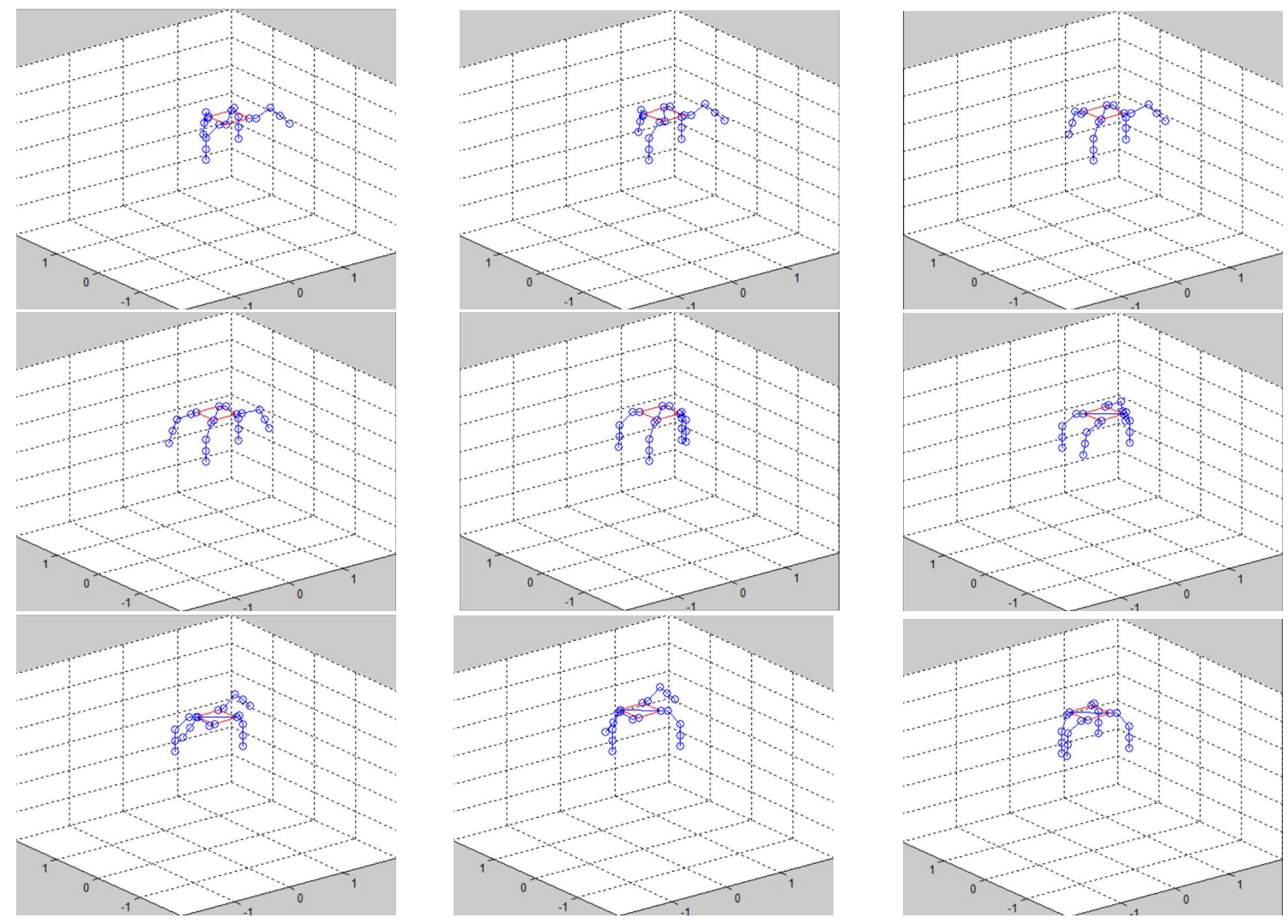

Figura G.1: Sequência de movimentos do Kamambaré sob a ação do algoritmo de otimização num ciclo completo de sua andadura. 


\section{Referências Bibliográficas}

Abderrahim, M.; Balaguer, C.; Gimenez, A.; Pastor, J. M.; Padrón, V. M. Roma: A climbing robot for inspection operations. Em IEEE International Conference on Robotics and Automattion, volume 3, p. 10-15, 1999.

Almeida, R. Z. H.; Hess-Coelho, T. A. Dynamic model of a 3-dof asymmetric parallel mechanism. The Open Mechanical Engineering Journal, 4, 2010.

Angeles, J. Fundamentals of Robotic Mechanical Systems, Theory, Methods, and Algorithms. Springer, 3ra edição, 2007.

Armada, M.; Santos, P. G. de; Jiménez, M. A.; Prieto, M. Application of CLAWAR machines. The International Journal of Robotics Research, 22:251-264, 2003.

Autumn, K.; Hsieh, S.T.; Dudek, D.M.; Chen, J.; Chitaphan, C.; Full, R. J. Dynamics of geckos running vertically. The Journal of Experimental Biology, (209): 260-272, 2006.

Bernardi, R.; Da Cruz, J. J. Kamanbaré: A Tree-climbing Biomimetic Robotic Platform for Environmental research. Em International Conference on Informatics in Control, Automation and Robotics (ICINCO), 2007.

Bernardi, R.; Potts, A. S.; Cruz, J.J. An automatic modelling approach to mobile robots. Em Troch, F. Breitenecker, editor, International Conference on Mathematical Modelling, volume CD, Vienna, 2009.

Betts, J.T. Survey of numerical methods for trajectory optimization. Guidance Control Dynamics, 21:193-207, 1998. 
Bobrow, J.E.; Park, F.C.; Sideris, A. Recent Advances on the Algorithmic Optimization of Robot Motion. Relatório técnico, Departament of Mechanical and Aerospace Engineering, University of California, 2004.

Brown, C.C.; Huissoon, J.P. Temporal gait control of a quadruped robot. Robotics and Autonomous Systems, 30:305-314, 2000.

Bryson, A.; Ho, Y. Applied Optimal Control. Optimization, Estimation and Control. John Wiley \& Sons, 1975.

Chettibi, T.; Haddada, M.; Labedb, A.; Hanchi, S. Generating optimal dynamic motions for closed-chain robotic systems. European Journal of Mechanics and Solids, 24:504-518, 2005.

Choi, H. R.; Ryew, S. M.; Kang, T. H.; Lee, J. H. KimH. M. A wall climbing robot with closed link mechanism. Em Proceedings of the 2000 IEEE RSJ International Conference on Intelligent Robots and Systems, 2000.

Choi, H.B.; Konno, A.; Uchiyama, M. Inverse dynamic analysis of a 4-dof parallel robot h4. Em Proceedings of 2004 IEEE/RSJ International Conference on Intelligent Robots and Systems, 2004.

Choi, H.S.; Park, Y.H. Development of a biped walking robot actuated by a closedchain mechanism. Robotica, 24:31-37, 2006.

Craig, J. Introduction to Robotics. Mechanics and Control. Addison Wesley Longman, 1989.

Eletronica-Industrial, . Bateria selada $12 \mathrm{v}$ 14ah. disponível em: <http://www.electronicaindustrial.pt>. Acesso em: 26 maio. 2011.

Espenschied, K. S.; Quinn, R. D.; Beer, R. D.; Chiel, H. J. Biologically based distributed control and local reflexes improve rough terrain locomotion in a hexapod robot. Robotics and Autonomous Systems, 1996.

EurAtiv.com, Robots to speak european. disponível em: $<$ www.euractiv.com/en/infosociety/robots-speak-european/article-145529>, Outubro 2005. Acesso em: 26 maio. 2011. 
Facchinei, F. Robust recursive quadratic programming algorithm model with global and superlinear convergence properties. Journal of Optimization Theory and Applications, 92:543-579, 1997.

Finotti, G. Cálculo Explícito dos torques dos atuadores de um robô paralelo plano empregando o Método de Kane. Tese de Doutorado, Escola Politécnica da Universidade de São Paulo, 2008.

Galvez, J. A.; Santos, P. G.; de Pfeiffer, F. Intrinsic tactile sensing for the optimization of force distribution in a pipe crawling robot. IEEE/ASME Transactions on Mechatronics, 6(1):26-35, 2001.

Giorgio, G.; Guerraggio, A.; Thierfelder, J. Mathematics of optimization: Smooth and nonsmooth case. Relatório técnico, Hardbound, 2004.

Goselin, C.; Angeles, J. Singularity analysis of closed-loop kinematic chains. IEEE Transactions on Robotics and Automation, 6:281-290, 1990.

Guo, L.S.; Zhang, Q. Adaptive trajectory control of a two dof closed-chain robot. Em Proceedings of the American Control Conference, 2001.

Harib, K.; Srinivasan, K. Kinematic and dynamic analysis of stewart platform-based machine tool structures. Robotica, 21:241-254, 2003a.

Harib, K.; Srinivasan, K. Kinematic ans dynamic analysis of stewart platform-based machine tool structures. Robotica, 21:541-554, 2003b.

Haynes, G. C.; Khripin, A.; Lynch, G.; Amory, J.; Saunders, A.; Rizzi, A.; Koditschek, D. Rapid pole climbing with a quadrupedal robot. Em IEEE International Conference on Robotics and Automation(ICRA 2009), 2009.

Haynes, G.C.; Rizzi, A.A. Gaits and gait transitions for legged robots. Em Proceedings of the 2006 IEEE International Conference on Robotics and Automation, 2006.

Henrici, P. Elements of Numerical Analysis. John Wiley \& Sons, 1ra edição, 1964.

Hildebrand, M. Vertebrate locomotion an introduction how does an animals body move itself along? Bioscience, 39, 1989. 
Hirose, S.; Kato, K. Study on Quadruped Walking Robot in Tokyo Institute of Technology - Past, Present and Future -. Proceedings of the 2000 IEEE Intemational Conference on Robotics and Automation, p. 414-419, 2000.

Hoffman, K. Analysis in Euclidean Space. Dover, 7ma edição, 2007.

Hull, D.G. Conversion of optimal control problems into parameter optimization problems. Guidance Control Dynamics, 20:57-62, 1997.

Hunt, K. H. Special configurations of robot-arms via screw theory. part 1. Robotica, 4:171-179, 1986.

Ingvast, J. Quadruped robot control and variable leg transmissions. Tese de Doutorado, KTH Industrial Engineering and Management, 2006.

Kane, T. R.; Levinson, D. A. Dynamics: Theory and Applications. Mcgraw Hill Series in Mechanical Engineering, 1985.

Khan, W. A.; Krovi, V.N.; Saha, S. K.; Angeles, J. Recursive kinematics and inverse dynamics for a planar 3r parallel manipulator. Journal of Dynamic Systems, Measurement, and Control, 127:529-536, 2005.

Kirk, D. Optimal Control Theory an Introduction. Dover Publication, 2da edição, 1998.

Kod $*$ Lab, University of Pennsylvania. The rise climbing robot. disponível em: $<$ www.kodlab.seas.upenn.edu>. Acesso em: 26 maio. 2011.

Kolter, J. Zico.; Rodgers, Mike P.; Ng, Andrew Y. A control architecture for quadruped locomotion over rough terrain. Relatório técnico, Computer Science Department, Stanford University, Stanford, 2008.

Kumar, V. Instantaneous kinematics of parallel chain robotic mechanisms. Em 21st Mechanisms Conf, p. 279-287, 1990.

Leech, J. W. Mecânica Analítica. Ao Livro Técnico S.A. and Editora da Universidade de São Paulo, 1971. 
Lin, S.K. Dynamics of the manipulator with closed chains. IEEE Transactions on Robotics and Automation, 6:496-501, 1990.

Luk, B.; Cooke, D.; Galt, S.; Collie, A.; Chen, S. Intelligent legged climbing service robot for remote maintenance applications in hazardous environments, 2005.

Luo, X.; Fan, X.; Zhang, H.; Chen, T. Integrated optimization of trajectories planning for robots manipulators based on intensified evolutionary programming. Em IEEE, International Conference on Robotics and Biomimetics, 2004.

Merlet, J.P. Parallel Robots (Solid Mechanics and Its Applications). Springer, 2da edição, 2006.

Murray, R. M.; Li, Z.; Sastry, S. S. A Mathematical Introduction to Robot Manipulation. CRC Press, 1994.

Pfeiffer, F.; Eltze, J.; Weidemann, H-J. The TUM walking machine. Intelligent Automation and Soft Computing. An International Journal, 1:307-323, 1995a.

Pfeiffer, F.; Eltze, J.; Weidemann, H-J. Six-legged technical walking considering biological principles. Robotics and Autonomous Systems, 14:223-232, 1995b.

Pieper, D.L. The kinematics of manipulators under computer control. Relatório técnico, Department of Mechanical Engineering, Stanford University, 1968.

Potts, A. S.; Da Cruz, J. J. Kinematics analysis of a quadruped robot. Em IFAC Symposium on Mechatronic Systems, Cambridge, Massachusetts, USA, 2010.

Potts, A. S.; Da Cruz, J. J. An optimal energy control algorithm for a moving platform. Em XVIII World Congress of the International Federation of Automatic Control (IFAC), Milão, Itália, 2011a.

Potts, A. S.; Da Cruz, J. J. Mobile Robots - Current Trends, chapter A Kinematical and Dynamical Analysis of a Quadruped Robot. INTECH, 1ra edição, 2011b.

Potts, A. S.; Da Cruz, J. J.; Bernardi, R. A first analysis of convergence for an iterative algorithm to minimize loss energy in mobile robots. Em Congresso Brasileiro de Automática, CBA, Juiz de Fora, Brasil, 2008a. 
Potts, A.S.; Da Cruz, J.J.; Bernardi, R. A quadratic programming approach to the minimum energy problem of a mobile robot. Em International Conference on Informatics in Control, Automations and Robotics, ICINCO, Ilha da Madeira, Portugal, 2008b.

Potts, A.S.; Da Cruz, J.J.; Bernardi, R. An iterative algorithm to minimize loss energy in mobile robot. Em IFAC Symposium on Robots Control, SYROCO, Gifu, Japan, 2009.

Robotics Electronics, Pololu. Tamiya 70103 universal gearbox kit. disponível em: <http://www.pololu.com/catalog/product/69/pictures $>$. Acesso em: 26 maio. 2011.

Robotics Lab, Hirose Fukushima. TITAN VII. disponível em: <wwwrobot.mes.titech.ac.jp>. Acesso em: 26 maio. 2011.

RobotWorx, . Robot history. disponível em: <http://www.robots.com/roboteducation.php?page=industrial+history >. Acesso em: 26 maio. 2011.

Santana, R.E.S. Projeto e um robô bípede para a reprodução da marcha humana. Dissertação de Mestrado, Escola Politecnica da Universidade de São Paulo, 2005.

Siegwart, R.; Nourbakhsh, I. R. Introduction to Autonomous Mobile Robots. The MIT Press, 2004.

Spong, M.; Vidyasagar, M. Robot Dynamics and Control. John Wiley \& Sons, 1ra edição, 1995.

Staicua, S.; Zhang, D. A novel dynamic modelling approach for parallel mechanisms analysis. Robotics and Computer-Integrated Manufacturing, 24:167-172, 2008.

Stryk, O.V.; Bulirsch, R. Direct and indirect methods for trajectory optimization. Em Annals of Operations Research, 1992.

Tartari, S.C; Cabral, E.L. Dynamics and jacobian analysis of a parallel architecture robot: The hexa. ABCM. Symposium series in Mechatronics, 2:166-173, 2006.

Tokhi, M.O.; Virk, G.S.; Hossain, M.A., editores. Climbing and Walking Robots. Springer, 2005. 
TrossenRobotics, . Rs-540 motor - 12v. disponível em: <http://www.trossenrobotics.com/store/p/5151-rs-540-motor-12v.aspx>.

Acesso em: 26 maio. 2011.

Tsai, L.W. Robot Analysis. The Mechanical of Serial and Parallel Manipulators. John Wiley \& Sons, 1ra edição, 1999.

Weingartent, J.D.; Lopes, G.A.D.; Buehler, M.; Groff, R.G.; Koditschek, D.E. Automated gait adaptation for legged robots. Em Proceedings of the 2004 IEEE International Conference on Robotics and Automation, 2004.

Winston, W. Introduction to Mathematical Programming. Applications and Algorithms. Duxbury Press, 4ta edição, 1995.

Xu, X. R.; Chung, W. J.; Choi, Y. H; Ma, X. F. A new dynamic formulation for robot manipulators containing closed kinematic chains. Robotica, 17:261-267, 1999 .

Zlatanov, D.; Fenton, R.G.; Benhabib, B. Singularity analysis of mechanisms and manipulators via a velocity-equation model of the instantaneous kinematics. IEEE Int. Conf. on Robotics and Automation, 1994a.

Zlatanov, D.; Fenton, R.G.; Benhabib, B. Singularity analysis of mechanisms and robots via a motion-space model of the instantaneous kinematics. IEEE Transactions on Robotics and Automation, p. 980-985, 1994b. 